Morphologic and Longitudinal Assessment in Thoracic Aortic Disease Management

Ignas B. Houben 
Colofon

Morphologic and longitudinal assessment in thoracic aortic disease management Ignas Bernardus Houben

ISBN/EAN: 978-94-6361-646-1

Copyright (c) 2022 Ignas Bernardus Houben

All rights reserved. No part of this thesis may be reproduced, stored or transmitted in any way or by any means without the prior permission of the author, or when applicable, of the publishers of the scientific papers.

Cover design by Steffen van Bergenhenegouwen

Lay-out and printing by Optima Grafische Communicatie 


\title{
Morphologic and Longitudinal Assessment in Thoracic Aortic Disease Management
}

\author{
Morfologische en longitudinale beoordeling in de \\ behandeling van thoracale aorta aandoeningen \\ (met een samenvatting in het Nederlands)
}

Proefschrift

ter verkrijging van de graad van doctor aan de Universiteit Utrecht op gezag van de rector magnificus, prof. dr. H.R.B.M. Kummeling, ingevolge het besluit van het college voor promoties in het openbaar te verdedigen op vrijdag 11 februari 2022 des middags te 4.15 uur

$$
\text { door }
$$

Ignas Bernardus Houben geboren op 26 april 1991 te Maastricht 
Promotores:

Prof. dr. J.A. van Herwaarden

Prof. dr. F.L. Moll

Financial support by the Stichting Concordia Trajectina for part of the research projects of this thesis is gratefully acknowledged.

Financial support by Chirurgisch Fonds UMC Utrecht and by the Dutch Heart Foundation for the publication of this thesis is gratefully acknowledged. 


\section{Contents}

\section{Part I: Introduction}

Chapter 1 Introduction and dissertation outline

\section{Part II: Type A aortic dissection assessment}

Chapter 2 Type A repair: managing more than just the entry tear Seminars of Thoracic and Cardiovascular Surgery

Chapter 3 Critical appraisal of multidimensional CT measurements following 31 acute open repair of type A aortic dissection Journal of Cardiac Surgery

Chapter 4 Endovascular ascending aortic repair in type A aortic dissection: a 49 systematic review Journal of Cardiac Surgery

\section{Part III: Descending thoracic aortic assessment}

Chapter 5 Thoracic endovascular aortic repair: a 16-year experience Submitted to ESVS

Chapter 6 Type B intramural hematoma: TEVAR or conservative approach? Annals of Cardiothoracic Surgery

Chapter 7 Thoracic endovascular aortic repair in the setting of compromised distal landing zones Annals of Thoracic Surgery

Chapter 8 Mapping pre-dissection aortic wall abnormalities: a multiparametric assessment European Journal of Cardiothoracic Surgery

\section{Part IV: Hemodynamic impact of graft repair}

Chapter 9 Left ventricular remodeling following aortic root and ascending aneurysm repair Journal of Thoracic and Cardiovascular Surgery

\section{Part V: Discussion}

Chapter 10 Discussion

Chapter 11 Discussie in het Nederlands

Appendix

- Review committee

- Acknowledgments

- About the author 



\section{Part I}

Introduction 

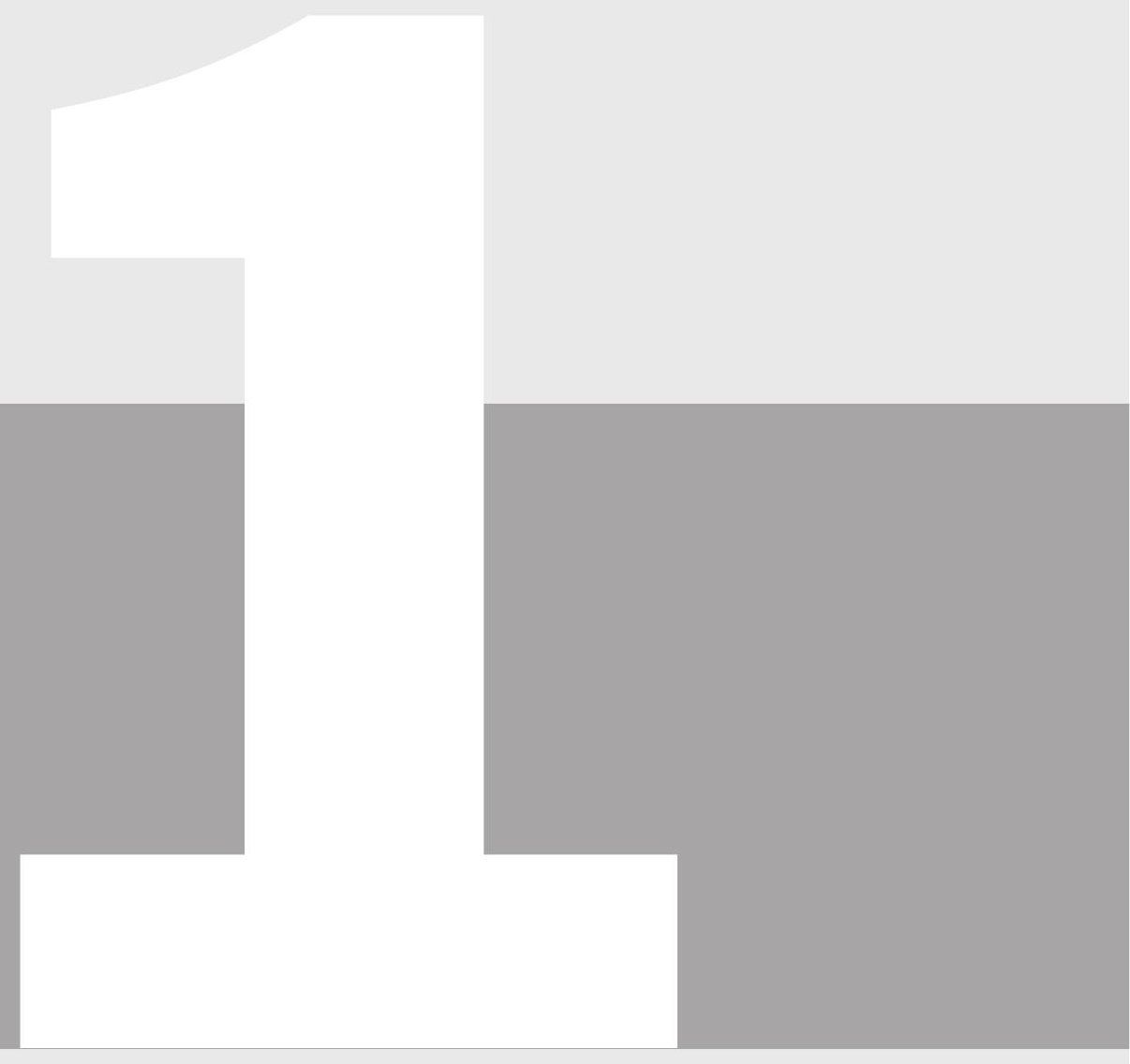
Introduction and dissertation outline 



\section{INTRODUCTION}

Thoracic endovascular aortic repair (TEVAR) is an established treatment for an array of aortic pathologies. Being introduced in the $1990 \mathrm{~s},{ }^{1,2}$ it has developed over roughly 25 years from a single and relatively simple tube applied in local descending thoracic aortic aneurysms, to modular advanced endovascular stent grafts for thoracic aortic aneurysms, dissections, ruptures, intramural hematomas, penetrating atherosclerotic ulcers, traumatic injuries, mycotic aneurysms and much more. The abdominal variant known as endovascular aortic repair (EVAR), swiftly outcompeted the open surgical aortic approach, so did TEVAR. Nowadays, approximately $90 \%$ of descending thoracic aortic pathologies is treated endovascularly rather than open. ${ }^{3-5}$ An evolution with one clear obvious advantage; minimal invasiveness. It has thus far shown to lead to reduced procedural length, lower morbidity, reduced hospital-stay, short-term lower mortality and cost-effectiveness. ${ }^{6-9}$ This is not all. With interventional cardiology now increasingly dominating a great deal of the valve repair market, ${ }^{10}$ the cardiovascular community is slowly creeping in on the one black box left in endovascular therapy: the ascending aorta.

This dissertation will highlight the different aspects of thoracic aortic disease assessment, with dissections and aneurysms in particular. The aims are to better understand the way we measure aortas and stent grafts, to better describe and predict any complications following TEVAR and to understand the hemodynamic consequences of TEVAR. This thesis is a critical appraisal of the challenges ahead in a management option which is increasingly dominating the aortic interventional armamentarium.

\section{DISSERTATION OUTLINE}

After the introduction and the outline of the thesis, the second part of this dissertation provides a contemporary analysis on type A aortic dissection. Chapter $\mathbf{2}$ provides an introduction to the challenges faced in type A aortic dissection repair and evaluates the extensiveness of the initial repair, considering the outcomes and interventional options for residual dissection. Subsequently, a critical appraisal of the measurements of the type $\mathrm{A}$ aortic dissection is reported in chapter $\mathbf{3}$, in order to get a better view on imaging assessment. Finally, in chapter 4, a systematic review is presented, describing all available literature on ascending aortic TEVAR in the case of type A aortic dissection.

In the third part, the role of TEVAR in the descending thoracic aorta is evaluated, starting with the introductory chapter $\mathbf{5}$ about TEVAR in descending thoracic intramural hematoma (IMH). This is followed by a single center 16-year experience report of long-term outcomes of TEVAR in chapter $\mathbf{6}$. Next, in chapter $\mathbf{7}$ the outcomes of TEVAR in a set 
of patients with a compromised distal landing zone are reported. Last, in chapter $\mathbf{8}$ a multi-parametric assessment of the descending thoracic aorta is presented, aiming to gain insights on pre-dissection wall abnormalities. This method uses a combination of three-dimensional growth mapping and computational dynamic mapping of transmural cyclic stress.

In part four, a clinical assessment of the hemodynamic impact following aortic graft repair is being described in two chapters. Chapter $\mathbf{9}$ focusses on left ventricular adverse remodeling following ascending aortic or root open repair with a Dacron graft.

Finally, the dissertation will be concluded and put into perspective with a discussion in Chapter 10, after which the dissertation is summarized in Dutch in Chapter 11. The appendices will outline the review committee, provide a list of publications, acknowledgements and a curriculum vitae. 
Introduction and dissertation outline

\section{REFERENCES}

1. Dake MD, Semba CP, Liddell RP, et al. Transluminal placement of endovascular stent-grafts for the treatment of descending thoracic aortic aneurysms. N Engl J Med. 1994;331(26):1729-1734. doi:10.1056/ NEJM199412293312601

2. Volodos NL, Karpovich I, Shekhanin V, et al. [A case of distant transfemoral endoprosthesis of the thoracic artery using a self-fixing synthetic prosthesis in traumatic aneurysm]. Grud Khir. 1987;(6):84-86. http://europepmc.org/abstract/med/3220297\%5Cnhttp://www.ncbi.nlm.nih.gov/pubmed/3220297. Accessed February 15, 2018.

3. Wang GJ, Jackson BM, Foley PJ, et al. CLINICAL RESEARCH STUDIES National trends in admissions, repair, and mortality for thoracic aortic aneurysm and type B dissection in the National Inpatient Sample. J Vasc Surg. 2018;67:1649-1658. doi:10.1016/j.jvs.2017.09.050

4. Geisbüsch S, Kuehnl A, Salvermoser M, et al. Increasing Incidence of Thoracic Aortic Aneurysm Repair in Germany in the Endovascular Era: Secondary Data Analysis of the Nationwide German DRG Microdata. Eur J VasC Endovasc Surg. 2019;57(4):499-509. doi:10.1016/j.ejvs.2018.08.013

5. Reutersberg B, Salvermoser M, Trenner M, et al. Hospital Incidence and In-Hospital Mortality of Surgically and Interventionally Treated Aortic Dissections: Secondary Data Analysis of the Nationwide German DiagnosisRelated Group Statistics From 2006 to 2014. J Am Heart Assoc. 2019;8(8). doi:10.1161/JAHA.118.011402

6. Gillen JR, Schaheen BW, Yount KW, et al. Cost analysis of endovascular versus open repair in the treatment of thoracic aortic aneurysms. In: Journal of Vascular Surgery. Vol 61. Mosby Inc.; 2015:596-603. doi:10.1016/j. jvs.2014.09.009

7. Jonker FHW, Trimarchi S, Verhagen HJM, et al. Meta-analysis of open versus endovascular repair for ruptured descending thoracic aortic aneurysm. J Vasc Surg. 2010;51(4):1026-1032.e2. doi:10.1016/j.jvs.2009.10.103

8. Gopaldas RR, Huh J, Dao TK, et al. Superior nationwide outcomes of endovascular versus open repair for isolated descending thoracic aortic aneurysm in 11,669 patients. J Thorac Cardiovasc Surg. 2010;140(5):10011010. doi:10.1016/j.jtcvs.2010.08.007

9. Patel HJ, Williams DM, Upchurch GR, et al. A Comparison of Open and Endovascular Descending Thoracic Aortic Repair in Patients Older Than 75 Years of Age. Ann Thorac Surg. 2008;85(5):1597-1604. doi:10.1016/j.athoracsur.2008.01.044

10. Still S, Szerlip M, Mack M. TAVR Vs. SAVR in Intermediate-Risk Patients: What Influences Our Choice of Therapy. Curr Cardiol Rep. 2018;20(10):18-23. doi:10.1007/s11886-018-1026-3 



\section{Part II}

Current status of type A aortic dissection management 


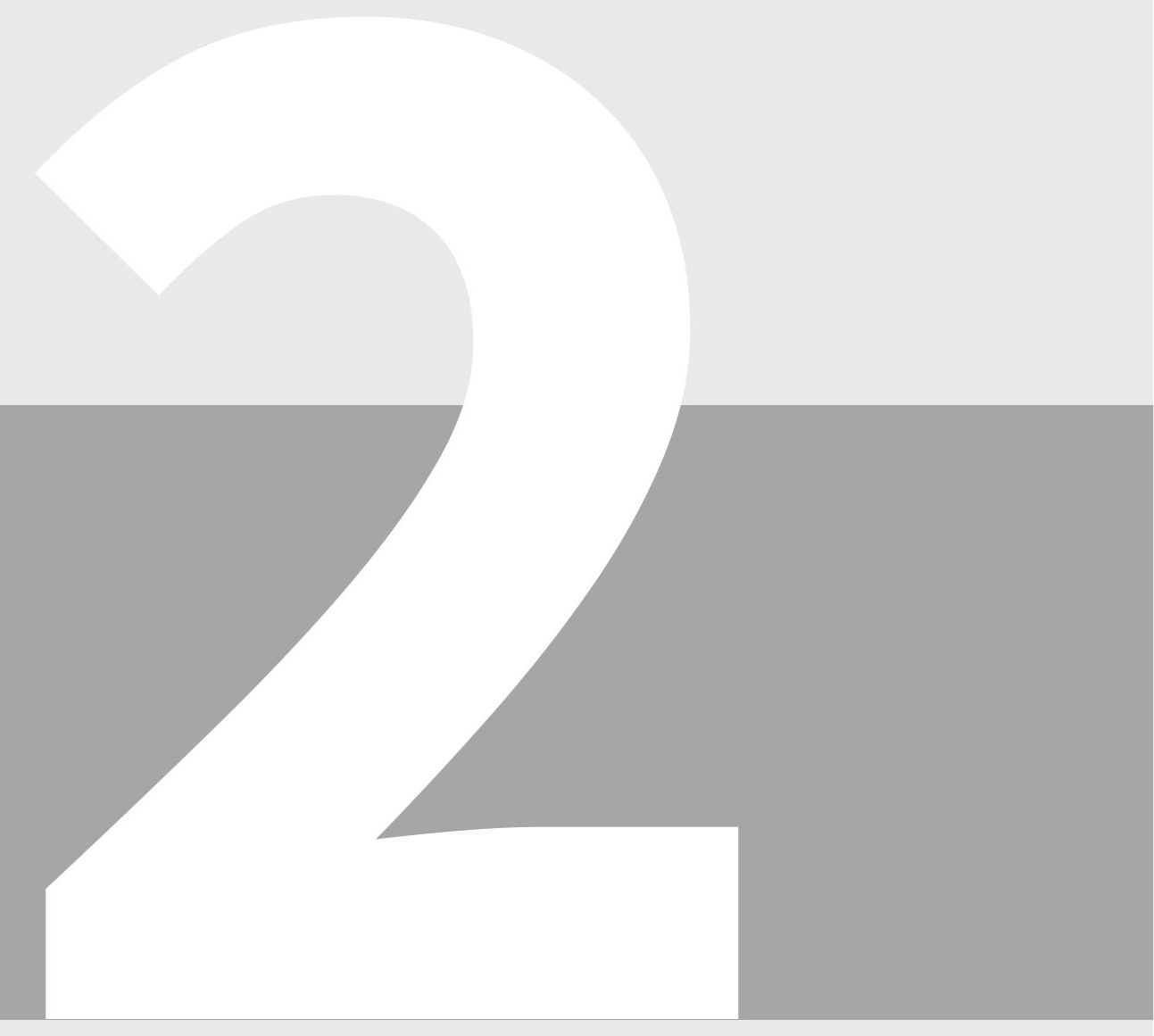




\section{Type A aortic dissection: managing more than just the entry-tear}

Ignas B. Houben, MD

Himanshu J. Patel, MD

Department of Cardiac Surgery, Frankel Cardiovascular Center, University of Michigan Health System, Ann Arbor, Michigan, United States

Semin Thorac Cardiovasc Surg. 2019;31(1):122-128. doi:10.1053/j.semtcvs.2018.08.011 


\section{GLOSSARY OF ABBREVIATIONS}

ATAAD -

CTA -

TEVAR -

FET

HCA acute type $A$ aortic dissection

computed tomography angiography

thoracic endovascular aortic repair

frozen elephant trunk

hypothermic circulatory arrest

\section{CENTRAL MESSAGE}

Identification of factors predicting distal aortic remodeling after repair of acute type $A$ dissection may facilitate decisions regarding extent and type of the initial repair in the endovascular era.

\section{Perspective statement}

Based on current clinical evidence, extensive initial distal aortic repair does not outweigh the benefits of conventional proximal aortic repair for all patients with acute type A aortic dissection. Finding early predictive factors for adverse aortic remodeling is key in the modern day treatment of acute type A aortic dissection.

\section{Central picture}

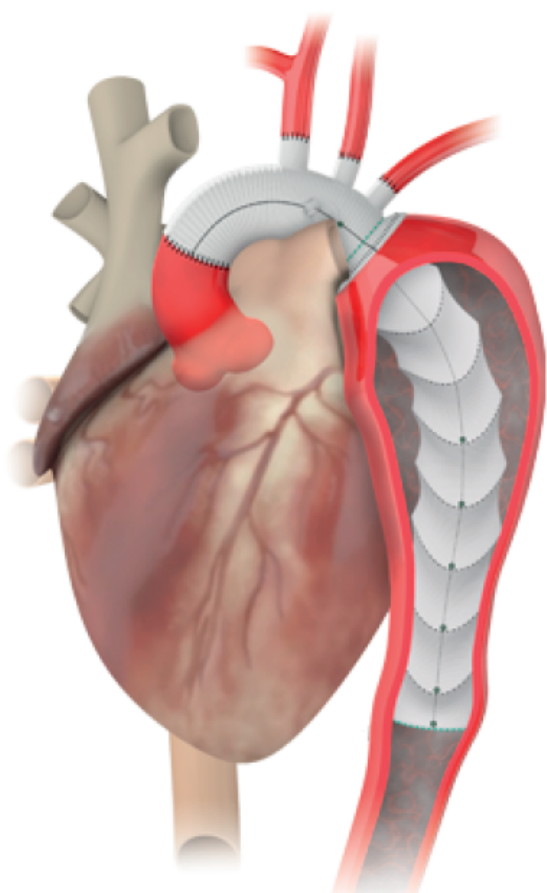




\section{INTRODUCTION}

Acute type A aortic dissection (ATAAD) is a lethal condition requiring immediate repair. ${ }^{1}$ Improved perioperative management has led to more patients surviving the initial hospitalization. ${ }^{2,3}$ Indeed, the in-hospital mortality has decreased significantly over the last 20 years from $31 \%$ to $17 \%$. ${ }^{2,3}$ Since most ATAAD patients have a DeBakey Type I configuration, the fate of the residual dissected aorta is becoming increasingly relevant for these survivors. Over $10 \%$ of repaired ATAAD cases require one or more additional surgical interventions due to residual distal dissection. ${ }^{4,5}$ Distal aortic reintervention remains a surgical challenge particularly if an additional open proximal approach is required for the aortic arch. ${ }^{6,7}$ It is for this reason that recent strategies have employed novel use of endovascular technology, including application either during the initial operation or early thereafter. Three options for the approach to a DeBakey type I dissection therefore exist and are reviewed here. First, ascending aortic replacement with adjunct hemiarch or extended arch replacement (proximal repair) and close postoperative computed tomography angiography (CTA) follow-up can be performed. Second, hybrid extensive proximal repair with a hemiarch or extended arch resection combined with antegrade descending thoracic endovascular aortic repair (TEVAR) as a frozen elephant trunk (FET) can be performed. Finally, proximal repair, combined with early elective retrograde TEVAR can be utilized. The choice between any of these three methods depends to a considerable extent on the surgeon's experience, preference and specific anatomic considerations. We provide a comprehensive overview of these management strategies and their success in treating aortic adverse remodeling based on current evidence of the literature.

\section{DISTAL AORTIC REMODELING}

Several retrospective trials have extensively studied the demographic, clinical and radiographic factors responsible for distal aortic dissection remodeling. The main risk factors, found for future aortic events have included younger age, Marfan syndrome, hypertension at follow-up, perioperative aortic diameter $>40 \mathrm{~mm}$ and patent or partially thrombosed false lumen (FL) after surgery. ${ }^{4,5,8}$ Dake et al. described a mnemonic-based classification system to divide patients into subsets according to anatomical involvement, with relevance for endovascular management (Table 1$).{ }^{9}$ This classification system aids in understanding the variances in clinical presentation of a challenging disease entity. It simplifies the assessment of the dissection for a clinician. However, it does not guide in predicting surgery outcomes, nor does it dictate the appropriate surgery. For type A dissection patients, some early factors that contribute to distal remodeling are known as mentioned earlier in this paragraph. Somehow, this does not change standard practice sufficiently and there is still a large group of patients where optimal treatment is unclear. 
Current status of type A aortic dissection management

Table 1 The DISSECT mnemonic classification published by Dake et al. ${ }^{9}$

\begin{tabular}{|c|c|c|}
\hline DISSECT & Definition & Expressed as \\
\hline Duration & $\begin{array}{l}\text { Duration of dissection as time from onset of } \\
\text { symptoms }\end{array}$ & $\begin{array}{l}\text { Acute }<2 \text { weeks } \\
\text { Subacute } 2 \text { weeks - } 3 \text { months } \\
\text { Chronic }>3 \text { months }\end{array}$ \\
\hline Intimal tear & Primary location intimal tear in the aorta & $\begin{array}{l}\mathbf{A}=\text { ascending aorta } \\
\mathbf{A r}=\text { aortic arch } \\
\mathbf{D}=\text { descending aorta } \\
\mathbf{A b}=\text { abdominal } \\
\text { Un = unknown }\end{array}$ \\
\hline Size & $\begin{array}{l}\text { Size of the aorta based on maximum trans-aortic } \\
\text { diameter measured by center line analysis }\end{array}$ & Size in $\mathrm{mm}$ \\
\hline $\begin{array}{l}\text { Segmental } \\
\text { extent }\end{array}$ & $\begin{array}{l}\text { Segmental extent of aortic involvement from } \\
\text { proximal to distal boundary }\end{array}$ & $\begin{array}{l}\mathbf{A}=\text { ascending aorta exclusively } \\
\mathbf{A r}=\text { aortic arch exclusively } \\
\mathbf{D}=\text { descending aorta exclusively } \\
\mathbf{A b}=\text { abdominal exclusively } \\
\mathbf{A a r}=\text { ascending to arch } \\
\mathbf{A D}=\text { ascending to descending } \\
\mathbf{A a b}=\text { ascending to abdomen } \\
\mathbf{A I}=\text { ascending to iliac } \\
\mathbf{A r D}=\text { arch to descending } \\
\mathbf{A r A b}=\text { arch to abdomen } \\
\mathbf{A r l}=\text { arch to iliac } \\
\mathbf{D A b}=\text { descending to abdomen } \\
\mathbf{D I}=\text { descending to iliac }\end{array}$ \\
\hline Clinical & Clinical complications related to dissection & $\begin{array}{l}\text { Aortic valve involvement } \\
\text { Cardiac tamponade } \\
\text { Progression + direction aortic involvement } \\
\text { Rupture } \\
\text { Branch vessel malperfusion }\end{array}$ \\
\hline Thrombosis & Thrombosis of aortic false lumen & $\begin{array}{l}\mathbf{P}=\text { patent aortic false lumen } \\
\mathbf{C T}=\text { complete thrombosis aortic false lumen } \\
\mathbf{P T}=\text { partial thrombosis aortic false lumen }\end{array}$ \\
\hline
\end{tabular}

\section{PROCEDURES}

The gold standard for the treatment of ATAAD is open surgical repair through median sternotomy. ${ }^{1}$ The procedure requires cardiopulmonary bypass with hypothermic circulatory arrest (HCA) and oftentimes either retrograde or antegrade cerebral perfusion. The primary goal of ATAAD repair is the prevention of a fatal rupture. Secondly, restoration of flow to the compromised branched vessels must be resolved and any aortic valve insufficiency or root pathology must be corrected if possible and deemed necessary. In order to achieve these goals, primary entry-tear location and aortic dimensions in the root, ascending and arch aorta require assessment. There are several technical approaches to manage this and each strategy has its own advantages, disadvantages and risks. 


\section{Proximal aortic repair only}

In the majority of patients with ATAAD the proximal tear can be fully resected by ascending aorta and hemiarch replacement. If the primary entry-tear cannot be found on imaging or intraoperative, the standard is still this proximal repair. Aortic valve repair is necessary in over $50 \%$ of patients and is combined with proximal repair. ${ }^{10}$ Aortic valve repair is beyond the scope of this review and will not be further discussed here. Early mortality rates for all proximal aortic repairs have continued to improve with reported rates from $4.4 \%$ to $9.9 \%$ at experienced sites and overall 1-year survival of more than $82 \%{ }^{8,11}$ If the intimal entry-tear or the dissection induced aneurysm extends to Ishimaru zone $0-2$, or if the dissection flap extends into the branch vessels, an extended or total arch repair, including repair of the origin of branch vessels may be necessary. In all other cases it is not clear whether ascending aorta with hemiarch repair has better outcomes than extended or total arch repair. Several studies have compared the two different approaches over the past two decades and no consensus exists identifying improved survival rates for either one of the two different approaches. (Table 2).${ }^{10,12}$ In addition, when freedom from distal aortic reintervention was evaluated in these studies, extended or total arch aortic repair was not consistently shown to be better than the conventional limited repair group. ${ }^{8,13,14}$

\section{Initial hybrid aortic repair}

In the past decade, several investigators have reported success of TEVAR for acute type $B$ aortic dissection. ${ }^{15,16}$ In these reports, early false lumen thrombosis led to favorable remodeling of the descending aorta, with the aorta often times demonstrating complete healing along the length of the treated aortic segment and beyond. Recognizing this successful application, TEVAR was then used in a hybrid manner to treat the distal aorta during acute type $A$ dissection. ${ }^{17}$ The reported early-mortality rates are similar to those described for a proximal repair strategy alone (Table 2) although hybrid repair may result in additional potential complications. These procedures are typically associated with longer cardiopulmonary bypass and circulatory arrest times. ${ }^{12,17}$ Secondly, sizing guidelines for landing zones in dissected aorta are not well understood, and oversizing can easily cause complications, such as infolding of the stent graft or stent graft-induced new entry tears. ${ }^{18}$ Both antegrade stenting and retrograde stenting approaches have been described in ATAAD repair, with the antegrade approach used most frequently. The current most commonly used hybrid repair is the FET. This single stage approach uses a stent graft of 10 to $15 \mathrm{~cm}$ deployed just beyond the left subclavian artery and preserves native mid arch aorta. ${ }^{19} \mathrm{~A}$ variant on this procedure was described by Roselli et al., where the stent graft is moved to a zone 2 location and a fenestration is created for the left subclavian artery where a branch vessel stent graft is placed..$^{20}$ Another one stage hybrid variant is Sun's procedure, first described in 2003. In this procedure a tetrafurcated 
Current status of type $\mathrm{A}$ aortic dissection management

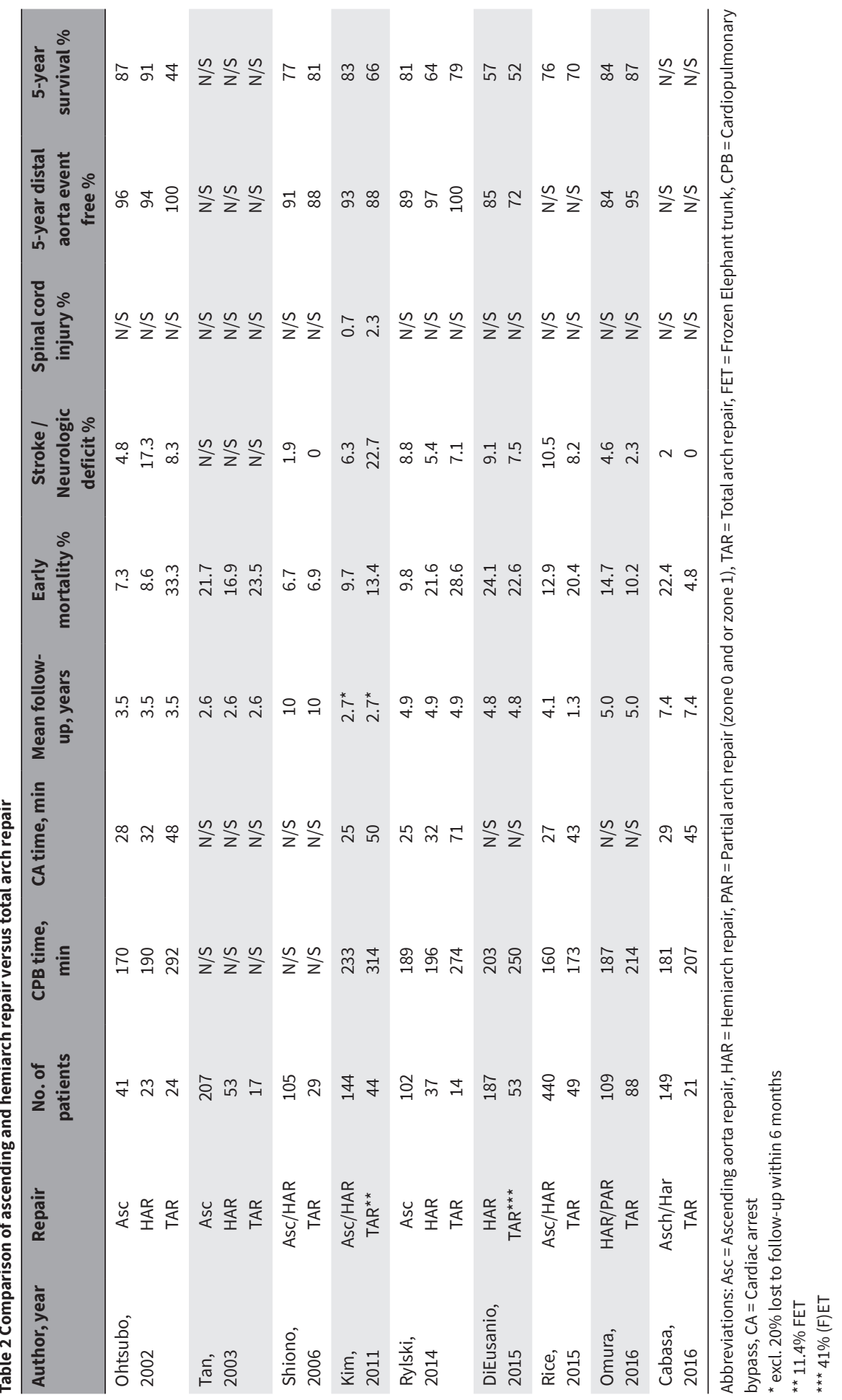


proximal graft providing three limbs to the branch arch vessels and a fourth perfusion limb are anastomosed to an antegrade placed stent graft at the level of the distal arch. ${ }^{11}$ Sun's procedure was the predecessor to many variants of an FET procedure, currently being introduced by clinicians in cooperation with medical device manufacturers.

\section{Proximal aortic repair and early TEVAR}

TEVAR has proven to be a safe and less invasive method to treat the dissected descending aorta and has successfully been used in zone 2 of the distal arch aorta. ${ }^{21}$ An alternative described strategy has been to treat the proximal aorta with a hemiarch or extended arch into zone 2, and perform distal TEVAR in a delayed fashion. Advantages of this approach include a potential reduction in cardiopulmonary bypass and circulatory arrest times at the initial procedure. Also, as this procedure is delayed to a time when hemodynamics are more stable, the risk for spinal cord ischemia may be reduced as well. Finally, if the initial operation is a proximal repair into Ishimaru zone 2, the sizing considerations for TEVAR at the proximal landing zone can then be simplified by landing the stent graft into a Dacron aortic graft. ${ }^{22}$

\section{COMPARISON OF CONSERVATIVE VERSUS HYBRID ENDOVASCULAR AORTIC REPAIR}

When recognizing that no consensus exists regarding extent of open proximal arch repair in acute type A dissection, the recent addition of hybrid distal procedures has only added to the confusion. No consistent improvement in late aortic or patient survival has been demonstrated with additional hybrid techniques, and new complications of higher spinal cord ischemic rates and stent graft induced entry-tears have now been introduced for a procedure typically done for immediate survival benefit., ${ }^{8,18}$ Several meta-analyses have not provided any clarification to guide clinical decisions. A 2015 meta-analysis included seven retrospective trials comparing these two strategies. ${ }^{23} 932$ patients were included, 503 with ascending and hemiarch repair and 429 with extensive repair, consisting of total arch repairs with different variants of proximal descending thoracic stent grafts. A subanalysis was performed with the 429 distal repair cases existing of 327 antegrade TEVARs, 19 retrograde TEVARs and 83 FETs. Overall comparison yielded no significant difference in operative mortality $(p=0.96)$, permanent neurologic deficit $(p=0.95)$ and late mortality $(p=0.59)$. However, the operative mortality odds ratio of extensive repair compared to limited repair was lower with $0.73(0.43-1.24,95 \%$ confidence interval). Additionally, an increase in spinal cord ischemia rate was seen in the adjunct distal repair group (odds ratio 1.34; 0.44- 4.04), though this finding was not statistically significant. Extensive repair significantly reduced the risk of distal reintervention compared to proximal repair (odds ratio $0.37 ; \mathrm{p}=0.01$ ), with a reintervention 
rate of $3.7 \%$ and $9.3 \%$ respectively. Subanalysis of the intraoperative extensive repair methods, showed a lower rate of distal reintervention and a higher rate of false lumen thrombosis with FET than with the TEVAR methods ( $p=0.008$ and $p<0.001$, respectively). Limitations of this study were the small number of included trials and the significant heterogeneity in the additional distal repair data.

A larger meta-analysis was published in 2016, and included 14 studies with 2221 patients. ${ }^{12}$ In this meta-analysis, trials comparing ascending and hemiarch repair to extended or total arch repairs with or without the combination of a hybrid distal thoracic repair were included. Again, no significant differences on in-hospital mortality were found between groups and the cardiopulmonary bypass time, cross clamping time and cardiac arrest time were significantly shorter in the limited repairs. Remarkably in this meta-analysis no significant difference was found in reintervention rates, although the rates again favored extensive repair ( $7.3 \%$ vs $3.3 \%$ ). The authors suggested that treatment bias was substantial, particularly since younger patients and patients with connective tissue disease where distal aortic growth is inevitable, were added to most of study populations. This report concluded that extensive arch repairs are safe, provided that they are carried out in high volume specialized centers. Total arch repair, is however not advocated as a primary approach by all centers and more data is needed to tailor extensive repair for the right patient group.

Finally, we conducted our own literature search to provide a short overview of results of studies comparing ascending and hemiarch repairs to full extensive aortic arch plus proximal descending repairs (Table 3 ). ${ }^{12,23}$ By doing this we were able to briefly assess more homogenous groups of treatment comparisons; limited repair vs total arch repair (Table 2) and limited repair vs full extensive repair (Table 3 ). The results described in Table 3 are similar to the ones in Table 2, although extensive aortic repair has an overall better outcome on reoperation rates compared to limited or total arch repair. ${ }^{12,23}$

\section{OUR PERSPECTIVE}

At the University of Michigan, we employ an entry tear guided approach for acute type A aortic dissection. As this review suggests, we believe this procedure should first be treated as a lifesaving one with a high risk for complications even with a conventional limited repair. We agree with the recent comments by Girardi suggesting that there remains selection bias in recent work evaluating different approaches for the arch and descending aorta, and that extrapolations should be carefully done when using this information to guide clinical decisions. ${ }^{24}$ However, we do have a low threshold for converting from a hemiarch repair to an extended proximal arch repair, and often do so 


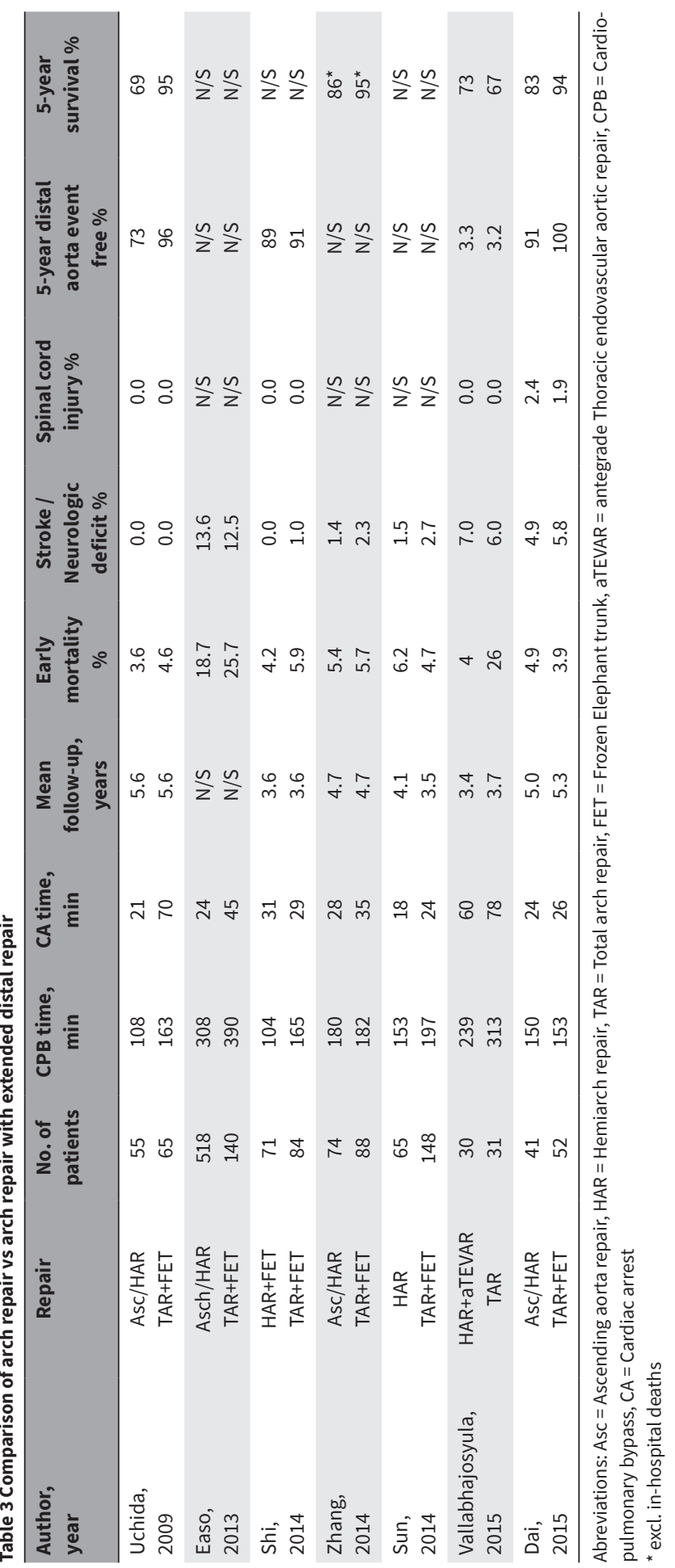


in the setting of a bovine arch configuration, or when the branch vessels are dissected. The frozen elephant trunk technique has been selectively used in the recent decade, particularly if an entry tear is identified in the distal arch, if the distal arch is ectatic, and finally in the setting of connective tissue disease where distal remodeling is inevitable. Sizing for TEVAR is based upon our personal experience, as well as a recent multicenter report. ${ }^{25}$ This work suggests that when the aorta acutely dissects, there is immediate expansion of the total diameter by approximately 10-20\% depending upon the location. We therefore use the total aortic diameter at the locations of the landing zones to determine the size of the frozen elephant trunk.

\section{CONCLUSION}

ATAAD repair outcomes are improving, but distal aortic remodeling remains an issue in a select group of patients. With newly developed technologies the issue of distal aortic remodeling can be addressed early to prevent the need for reintervention. This early aggressive approach may be useful, as reintervention in a chronic dissection patient has proven to be challenging with variable outcomes. However, early aggressive approach comes at a price and has not yet proven to be more beneficial, compared to limited repair and watchful waiting. It will be imperative to develop a patient-specific early stage prediction model for residual aortic reintervention. 
Type A repair: managing more than just the entry tear

\section{REFERENCES}

1. Hiratzka LF, Bakris GL, Beckman JA, et al. 2010 ACCF/AHA/AATS/ACR/ASA/SCA/SCAI/SIR/STS/SVM guidelines for the diagnosis and management of patients with Thoracic Aortic Disease: a report of the American College of Cardiology Foundation/American Heart Association Task Force on Practice Guidelines, A. Circulation. 2010;121(13):e266-369. doi:10.1161/CIR.0b013e3181d4739e.

2. Pape LA, Awais M, Woznicki EM, et al. Presentation, diagnosis, and outcomes of acute aortic dissection: 17year trends from the international registry of acute aortic dissection. J Am Coll Cardiol. 2015;66(4):350-358. doi:10.1016/j.jacc.2015.05.029.

3. Knipp BS, Deeb GM, Prager RL, Williams CY, Upchurch GR, Patel HJ. A contemporary analysis of outcomes for operative repair of type A aortic dissection in the United States. Surgery. 2007;142(4):524-528. doi:10.1016/j. surg.2007.07.012.

4. Halstead JC, Meier M, Etz C, et al. The fate of the distal aorta after repair of acute type A aortic dissection. J Thorac Cardiovasc Surg. 2007;133(1):127-135. doi:10.1016/j.jtcvs.2006.07.043.

5. Leontyev S, Haag F, Davierwala PM, et al. Postoperative Changes in the Distal Residual Aorta after Surgery for Acute Type A Aortic Dissection: Impact of False Lumen Patency and Size of Descending Aorta. Thorac Cardiovasc Surg. 2017;65(2):90-98. doi:10.1055/s-0036-1571813.

6. Rylski B, Beyersdorf F, Desai ND, et al. Distal aortic reintervention after surgery for acute DeBakey type I or II aortic dissection: Open versus endovascular repair. Eur J Cardio-thoracic Surg. 2015;48(2):258-263. doi:10.1093/ ejcts/ezu488.

7. Dell'Aquila AM, Pollari F, Fattouch K, et al. Early outcomes in re-do operation after acute type A aortic dissection: results from the multicenter REAAD database. Heart Vessels. 2017;32(5):566-573. doi:10.1007/s00380-0160907-x.

8. Kim JB, Chung CH, Moon DH, et al. Total arch repair versus hemiarch repair in the management of acute DeBakey type I aortic dissection. Eur J Cardio-thoracic Surg. 2011;40(4):881-887. doi:10.1016/j.ejcts.2010.12.035.

9. Dake MD, Thompson M, Van Sambeek M, Vermassen F, Morales JP. DISSECT: A new mnemonic-based approach to the categorization of aortic dissection. Eur J Vasc Endovasc Surg. 2013;46(2):175-190. doi:10.1016/j. ejvs.2013.04.029.

10. Cabasa A, Pochettino A. Surgical management and outcomes of type A dissection-the Mayo Clinic experience. Ann Cardiothorac Surg. 2016;5(4):296-309. doi:10.21037/acs.2016.06.01.

11. Sun L, Qi R, Zhu J, Liu Y, Zheng J. Total arch replacement combined with stented elephant trunk implantation: A new standard therapy for type a dissection involving repair of the aortic arch? Circulation. 2011;123(9):971-978. doi:10.1161/CIRCULATIONAHA.110.015081.

12. Poon SS, Theologou T, Harrington D, Kuduvalli M, Oo A, Field M. Systematic Review Hemiarch versus total aortic arch replacement in acute type A dissection: a systematic review and meta-analysis. Ann Cardiothorac Surg. 2016;5(4):156-173. doi:10.21037/acs.2016.05.06.

13. Shiono M, Hata M, Sezai A, Niino T, Yagi S, Negishi N. Validity of a Limited Ascending and Hemiarch Replacement for Acute Type A Aortic Dissection. Ann Thorac Surg. 2006;82(5):1665-1669. doi:10.1016/j.athoracsur.2006.05.112.

14. Di Eusanio M, Berretta P, Cefarelli M, et al. Total Arch Replacement Versus More Conservative Management in Type A Acute Aortic Dissection. Ann Thorac Surg. 2015;100(1):88-94. doi:10.1016/j.athoracsur.2015.02.041.

15. Nienaber CA, Kische S, Rousseau H, et al. Endovascular repair of type B aortic dissection: Long-term results of the randomized investigation of stent grafts in aortic dissection trial. Circ Cardiovasc Interv. 2013;6(4):407-416. doi:10.1161/CIRCINTERVENTIONS.113.000463.

16. Brunkwall J, Kasprzak P, Verhoeven E, et al. Endovascular repair of acute uncomplicated aortic type b dissection promotes aortic remodelling: 1 year results of the ADSORB trial. Eur J Vasc Endovasc Surg. 2014;48(3):285291. doi:10.1016/j.ejvs.2014.05.012.

17. Easo J, Weigang E, HölzI PPF, Horst M, Hoffmann I, Blettner M OED for the GSG. Influence of Operative Strategy for the Aortic Arch in De Bakey Type I Aortic Dissection - Analysis of the German Registry for Acute Aortic Dissection Type A (GERAADA). Ann Cardiothorac Surg. 2013;2(2):175-180. doi:10.3978/j.issn.2225-319X.2013.01.03. 
Current status of type A aortic dissection management

18. Huang CY, Chen CW, Chen PL, et al. Association between Aortic Remodeling and Stent Graft-Induced New Entry in Extensive Residual Type A Dissecting Aortic Aneurysm after Hybrid Arch Repair. Ann Vasc Surg. 2016;31:60-69. doi:10.1016/j.avsg.2015.08.022.

19. Pochettino A, Brinkman WT, Moeller P, et al. Antegrade Thoracic Stent Grafting During Repair of Acute DeBakey I Dissection Prevents Development of Thoracoabdominal Aortic Aneurysms. Ann Thorac Surg. 2009;88(2):482490. doi:10.1016/j.athoracsur.2009.04.046.

20. Roselli EE, Tong MZ, Bakaeen FG. Frozen elephant trunk for DeBakey type 1 dissection: the Cleveland Clinic technique. Ann Cardiothorac Surg. 2016;5(3):251-255. doi:10.3978/10603.

21. Kamman A V., Williams DM, Patel HJ. Thoracic Endovascular Aortic Repair into the False Lumen in Chronic Aortic Dissection. Ann Vasc Surg. 2017;42:303.e11-303.e14. doi:10.1016/j.avsg.2016.11.027.

22. Doi T, Sakata K, Gyoten T, et al. Two-Stage Hybrid Repair in a Patient with Acute Type A Aortic Dissection Associated with Right Aortic Arch with Aberrant Left Subclavian Artery Originating from a Kommerell Diverticulum. Ann Vasc Dis. 2016;9(3):223-227. doi:10.3400/avd.cr.16-00013.

23. Settepani F, Cappai A, Basciu A, et al. Hybrid versus conventional treatment of acute type A aortic dissection. $J$ Card Surg. 2015;30(9):707-713. doi:10.1111/jocs.12598.

24. Girardi LN. Total arch for type A dissection? J Thorac Cardiovasc Surg. 2016;151(2):294-295. doi:10.1016/j. jtcvs.2015.03.058.

25. Rylski B, Blanke P, Beyersdorf F, et al. How Does the Ascending Aorta Geometry Change When It Dissects? J Am Coll Cardiol. 2014;63(13):1311-1319. doi:10.1016/j.jacc.2013.12.028. 



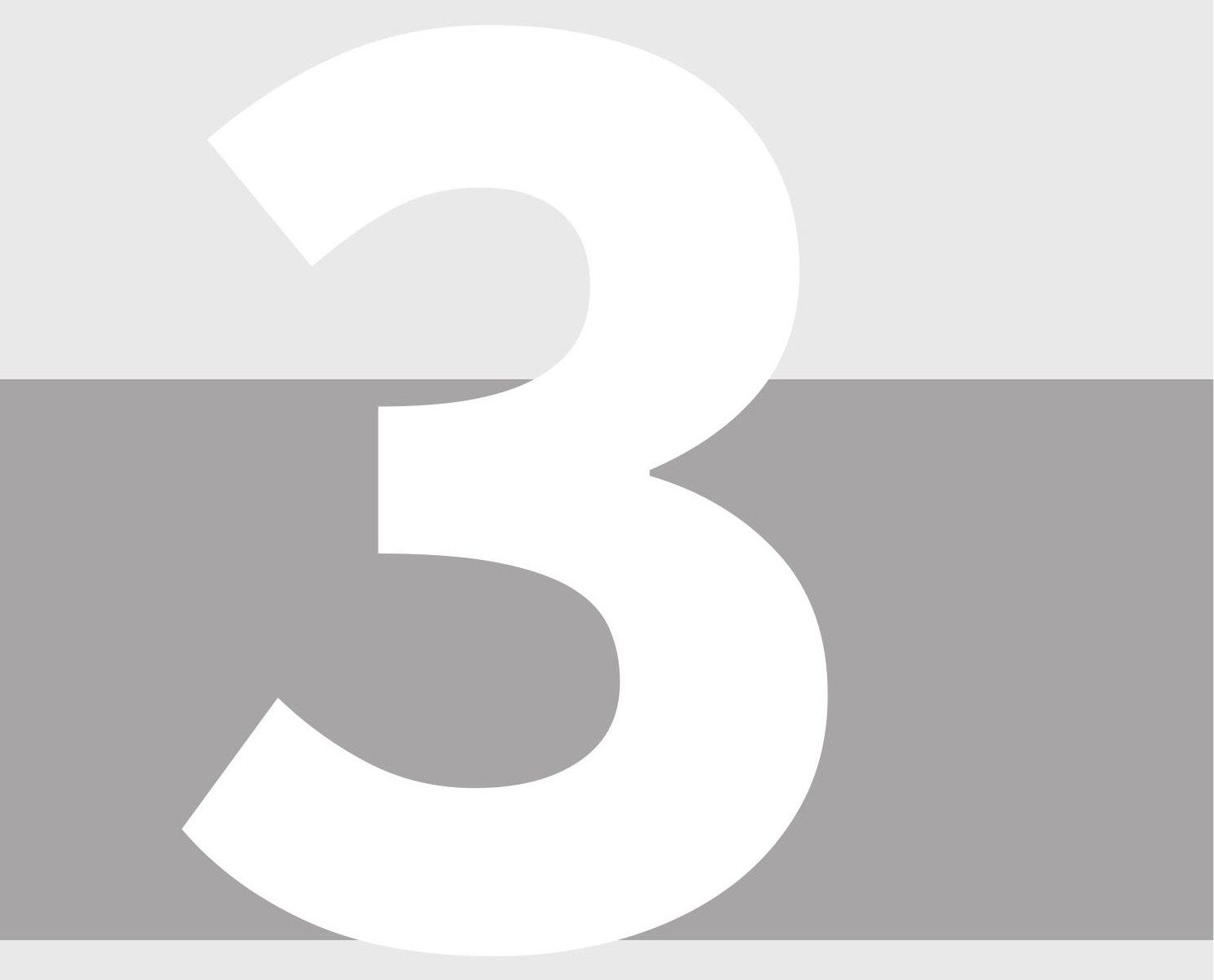




\section{Critical appraisal of multidimensional CT measurements following acute open repair of type $A$ aortic dissection}

Ignas B. Houben ${ }^{1} \mathrm{MD}$, Theodorus M.J. van Bakel ${ }^{2}$ MD PhD, Nicholas S. Burris ${ }^{3}$ MD, Frans L. Moll ${ }^{4}$ MD PhD, Joost A. van Herwaarden ${ }^{4}$ MD PhD, Himanshu J. Patel ${ }^{1}$, MD.

${ }^{1}$ Department of Cardiac Surgery, Frankel Cardiovascular Center, University of Michigan, Ann Arbor, United States ${ }^{2}$ Departments of Surgery \& Biomedical Engineering, University of Michigan, Ann Arbor, United States ${ }^{3}$ Department of Radiology, University of Michigan, Ann Arbor, United States

${ }^{4}$ Department of Vascular Surgery, Utrecht University, Utrecht, The Netherlands 


\section{ABSTRACT \\ Introduction}

To identify patients with aneurysmal degeneration of the native aorta following type A aortic dissection (TAAD), reproducible serial measurements of aortic dimensions are critical. We used a systematic workflow for measuring aortic geometry following TAAD, using computed tomography angiography (CTA) data, and test its reproducibility.

\section{Methods}

The workflow for aortic measurements included center line generation, luminal diameter and area measurement at six anatomically defined locations along the aorta and luminal volumetric measurements in the descending aorta. Two independent observers measured the aortic geometry in 20 surgically repaired TAAD patients, preoperatively and at three months follow-up. To test reproducibility, intra- and interobserver agreement scores were analyzed using a concordance correlation coefficient (CCC).

\section{Results}

The interobserver agreement scores of the diameter, area and volumetric measurements in the descending aorta were acceptable. The agreement scores of the area measurements were highest, with CCCs ranging 0.909-0.984. Luminal diameter measurements scored lower than luminal area measurements and were least reproducible at the mid aortic arch $(\mathrm{CCC}<0.886)$. Overall, intraobserver agreement scores were better than interobserver agreement scores (SD of mean difference was 1.89 vs 1.94 for intra- vs interobserver diameter measurements, and 0.61 vs 0.66 for area measurements).

\section{Conclusion}

Although overall reproducibility was acceptable in descending aortic measurements, our results show that it remains challenging to reliably measure luminal diameters, compared to areas. In order to aid identification of early adverse remodeling following acute TAAD, novel two- and three-dimensional measurement techniques are needed that capture locoregional changes in FL and TL morphology more accurately. 


\section{INTRODUCTION}

Currently, $80-90 \%$ of type A aortic dissection (TAAD) patients who make it to the hospital, survive the first 30 days following repair. ${ }^{1,2}$ More than $10 \%$ of these patients will require surgical reintervention during follow-up, most commonly due to adverse remodeling and dilation of the FL. ${ }^{3-5}$ Recent registry data has shown that thoracic endovascular aortic repair (TEVAR) in the subacute phase following aortic dissection ( 2 weeks - 3 months) yields a lower mortality rate and significantly larger degree of positive aortic remodeling compared to endovascular repair in the chronic phase. ${ }^{6}$ This difference has been attributed to thickening and stiffening of the intimal flap over time, ${ }^{7}$ increasing the risk of endograft related complications. ${ }^{8}$ Following these results, early detection of adverse aortic remodeling is desirable to identify patients who will require aortic repair during follow-up. ${ }^{9}$ Currently, diameter measurements of the aortic lumen are the mainstay measurement technique for the assessment of aortic geometry over time. Additionally, luminal area and volumetric measurements can be obtained from computed tomography angiography (CTA) image data. In recent years, various measurement techniques have been used to define endpoints in studies analyzing the effectiveness of different techniques for aortic repair. ${ }^{10-13}$ However, these studies all use a different approach for measuring aortic morphology, and the inter- and intra-observer agreement scores are not reported, making their reproducibility questionable.

In order to compare the results of different studies analyzing the morphologic changes following acute TAAD, a systematic workflow for measuring aortic morphology following TAAD is needed. In the present study, we used a systematic workflow for measuring aortic geometry following acute TAAD using two-dimensional and three-dimensional measurement techniques, and test its reproducibility.

\section{METHODS}

Approval for this study was obtained from the institutional review board (University of Michigan protocol number HUM00061722, date of approval 5/21/2012), the need for patient consent was waived. The University of Michigan cardiac surgery database was retrospectively queried to identify 20 TAAD patients (DeBakey type I), who had available preoperative and three-month postoperative CTA examinations. CTA examinations were acquired on a multi-slice scanner after intravenous injection of $120 \mathrm{~mL}$ iopamidol intravenous contrast (Isoview 370, Bracco Diagnostics, Milan, Italy) and prospective reconstruction was performed in the mid-diastolic phase. (75\% of the R-R interval). The CTA image data were analyzed using automatic image processing tools in the software package Vitrea Core (Vital Images Inc., Product Version 6.9.1, Minnetonka, MN, USA). All measurements were performed by two observers, TvB and IH. For intraobserver mea- 
surements, an interval between measurements of at least two weeks was followed and scans were randomly re-ordered in between measurement intervals in order to avoid pattern recognition.

\section{Image processing}

In the following, the systematic workflow for measuring diameters, areas and volumes in the regions of interest is reported. Figure 1 presents a visualization of the different steps in our workflow. First, CTA imaging data was imported in Vitrea Core. Then, a center line (CL) of the whole aorta, including FL and TL, was manually generated starting at the level of the aortic valve and ending at the aorto-iliac bifurcation. Subsequently, a curved multiplanar reformatted image was generated to confirm central position of the $\mathrm{CL}$ within the total aortic perimeter. If needed, manual adjustments were made in areas of high tortuosity. Using planes perpendicular to the $\mathrm{CL}$, regions of interest (ROIs) were manually drawn to obtain diameter in $\mathrm{mm}$ and luminal area in $\mathrm{cm}^{2}$. ROls were drawn delineating the total aortic lumen (outer wall of the aorta including both TL and FL), and the separate luminal areas. Intraluminal calcifications were included in the ROI. Ellipticity was defined as the largest diameter of the lumen divided by the smallest diameter. The above mentioned measurements were obtained in six locations along the CL: (1) the mid ascending aorta (midpoint between the aortic valve and the origin of the innominate artery); (2) the mid aortic arch (midpoint between left common carotid and subclavian arteries); (3) the proximal descending aorta [2 $\mathrm{cm}$ distal to distal end of the left subclavian artery (LSA)]; (4) the mid descending aorta $(10 \mathrm{~cm}$ distal to distal end of the LSA); (5) the distal descending aorta ( $2 \mathrm{~cm}$ proximal to proximal end of celiac trunk) and (6) infrarenal (1.5 cm distal to the most inferior renal artery). TL and FL volume measurements were obtained using a semi-automated threshold-based segmentation tool with manual adjustments where needed. Areas of FL thrombus were included in the volume measurements. Both volumes were measured in the descending thoracic aorta, starting just distal to the origin of the LSA and ending just proximal to the origin of the celiac trunk.

\section{Sample size calculation}

Sample size calculations were based on a $\rho 0$ (H0 lowest acceptable concordance correlation coefficient) of 0.9 and a $\rho 1$ ( $\mathrm{H} 1$ expected outcome of concordance correlation coefficient) of 0.95 , yielding a required sample size of $n=32$ observations using a significance $(\alpha)=0.05$ and a power $(1-\beta)$ of $0.80 .{ }^{14}$ In our case 40 observed scans from 20 patients were used. As the morphology pre- and post-surgical intervention affected the morphology significantly, we considered all 40 scans to be independent. 
Figure 1. Measurement workflow

\section{Centerline}

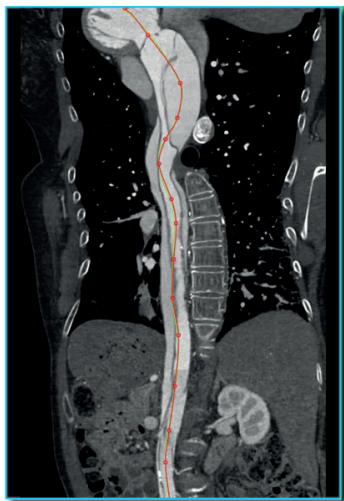

2. Diameter/Area

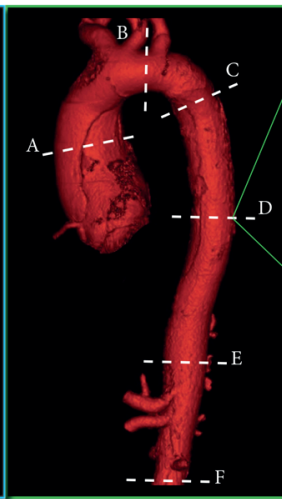

$\mathrm{A}-\mathrm{F}=$ diameter and area measurement locations SLAREa/TL Area mm²

The workflow of our measurements depicting (1) manual centerline generation, (2) diameter and area measurements at six locations (A-F), and (3) volumetric measurements using a vessel growth tool. FL, false lumen; TL, true lumen

\section{Statistical analysis}

Normal distribution of the continuous data was tested using the Shapiro-Wilk test. Logarithmic transformation was used to achieve normal distribution of the data where necessary. The $95 \%$ limits of agreement were defined as the mean difference \pm repeatability coefficient (SD*1.96). These results were visually depicted using Bland-Altman plots. A priori acceptable differences for diameter, luminal area and volumetric measurements were added to the Bland-Altman plots. These were respectively defined as $3 \mathrm{~mm}, 1$ $\mathrm{cm}^{2}$, and $30 \mathrm{~mL}$, based on clinical expertise and earlier work for type B aortic dissection (TBAD). ${ }^{15}$ Intra- and interobserver agreement were analyzed using a concordance correlation coefficient (CCC). ${ }^{16} \mathrm{CCC}$ values $<0.90$ were considered "poor agreement", between 0.90-0.95 "moderate", 0.95-0.99 "substantial" and >0.99 "almost perfect" as described by Lin et al. ${ }^{17}$ Continuous data are presented using mean \pm SD. P-values $<0.05$ were considered statistically significant. All tests were performed using SPSS version 24.0 (IBM SPSS Statistics, Armonk, NY).

\section{RESULTS}

Among the 20 included patients, ten were male (50\%). The mean age was $60.4 \pm 12.1$ years. All acute TAAD patients received surgical repair within fourteen days after onset of dissection. Surgery extended to zone 0 in ten (50\%) patients and to zone 1 in ten (50\%) patients and the ascending aortic graft ranged 24 to $30 \mathrm{~mm}$ in diameter. Mean follow-up duration at the postoperative analysis was $104.0 \pm 24.5$ days. The combined diameter and area assessment over the dissected areas showed higher agreement for intraobserver (CCC = $0.894)$ vs interobserver $(\mathrm{CCC}=0.881)$ measurements. Overall, intraobserver variability was 
lower than interobserver variability for diameter measurements (SD of mean difference of 1.89 vs 1.94 for intra- vs interobserver) and for area measurements (SD of mean difference of 0.61 vs 0.66 ). The difference between intra- and interobserver measurement variability is shown in Figure 2. The results of the interobserver reproducibility analysis will be the focus of the remainder of this work, as the higher degree of interobserver variability will drive the overall reliability of our measurement workflow in a typical clinical situation.

Figure 2. Intra- vs interobserver Bland-Altman plots

Intraobserver

A

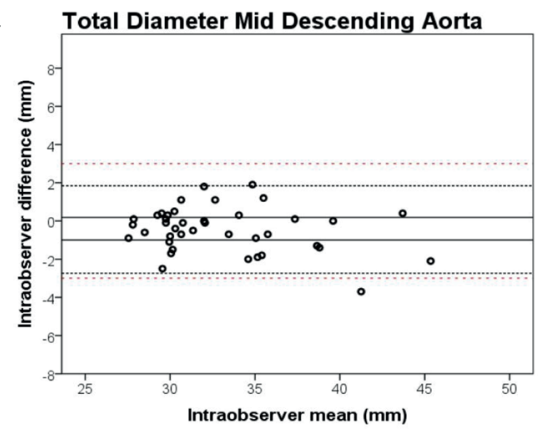

C

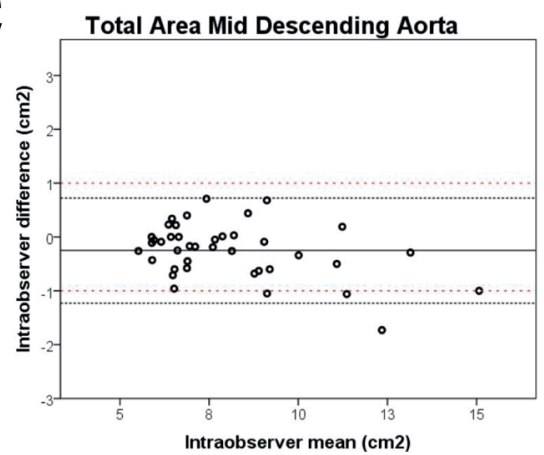

Interobserver

B

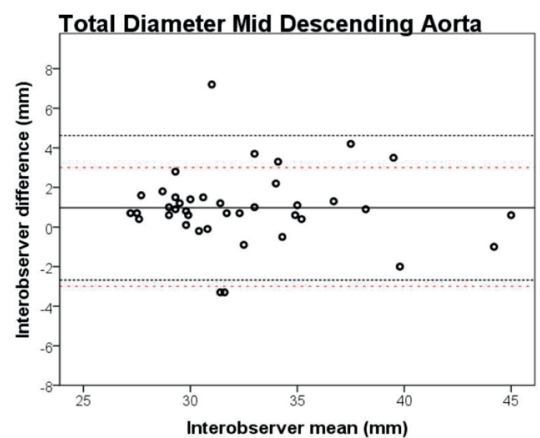

$\mathrm{D}$

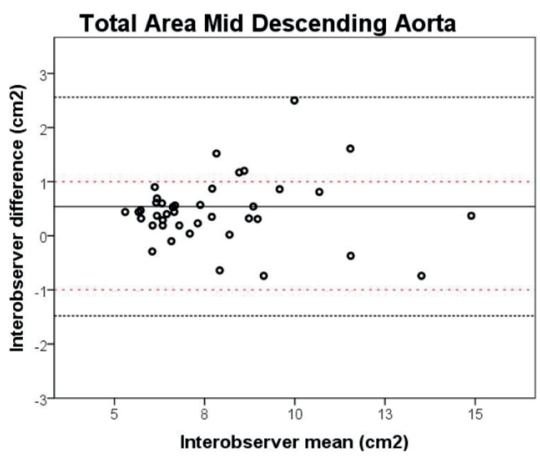

Intra- vs interobserver Bland-Altman plots for maximum total aortic diameter measurements (A vs B) and for maximum total area measurements ( $C$ vs D) at the mid descending aorta. The solid black line represents the mean of all measurements paired. The black close dotted lines represent the limits of agreement and the red double spaced dotted line represents the a priori defined acceptable differences. The limits of agreement are defined within range of the a priori acceptable differences when the black dotted lines both do not exceed the red dotted lines. The variability is higher for interobserver ( $B$ and $D$ ) than for intraobserver ( $A$ and $C$ ) measurements.

\section{Aortic growth}

Table 1 shows high variability in the luminal diameter growth assessment of the descending aorta, particularly on FL growth assessment in the proximal descending aorta, being $-1.02 \pm 14.41 \mathrm{~mm}$. The FL area growth assessment in the proximal descending aorta is positive, showing $1.66 \pm 1.23 \mathrm{~cm}^{2}$. 


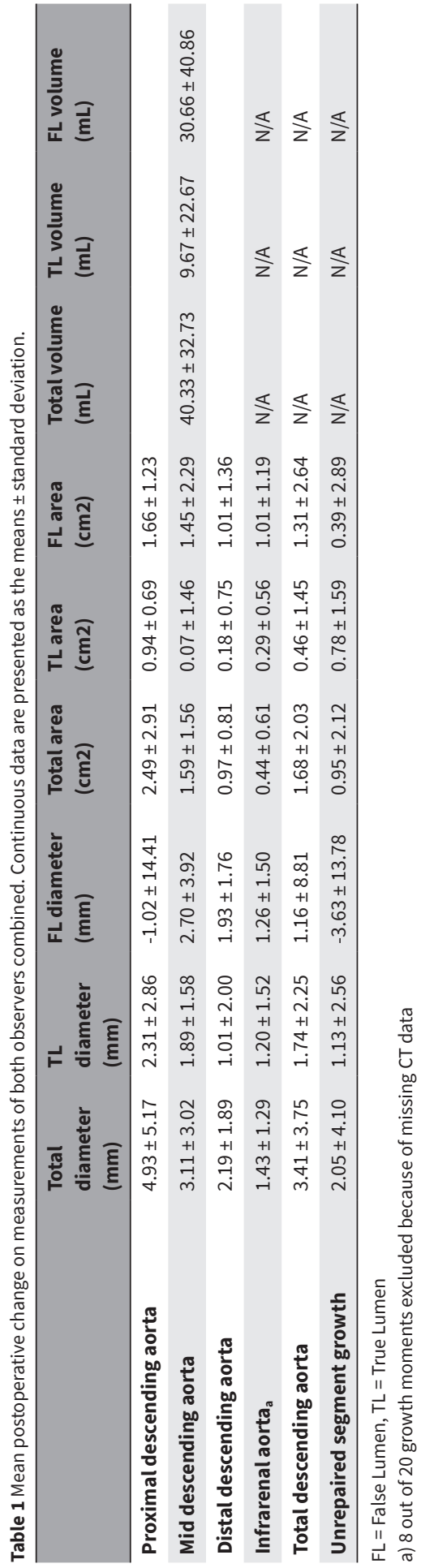




\section{Aortic diameter and area agreement}

A post repair analysis of the ascending aorta revealed acceptable limits of agreement based on our a priori definitions (Figure 3A, Table 2). Since the repaired segment of the ascending aorta was expected to have stable diameters with good contrast enhancement and no false lumen, the Bland-Altman plot was used as a baseline comparison (Figure 3A).

Of all six aortic locations, the measurements of aortic arch total diameter showed the largest mean difference $(1.09 \mathrm{~mm})$ and largest SD of the mean difference $(2.58 \mathrm{~mm}$, Figure $3 \mathrm{~B})$. Aortic arch agreement scores for diameter and area measurements were overall low, with poorest agreement in the measurements of the true and false lumen maximal diameter (CCC $=0.886$ and 0.820 ) (Figure 3C, Table 3). The total aortic area agreement score at the level of the aortic arch was lowest compared to all other locations, with a moderate CCC of 0.907 (Table 3).

Figure 3. Pre- and postoperative diameter measurement interobserver Bland-Altman plots

A

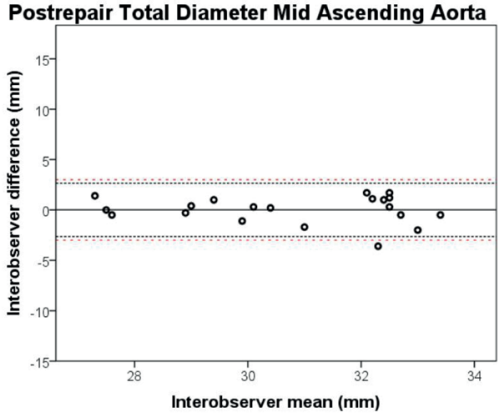

$\mathrm{C}$

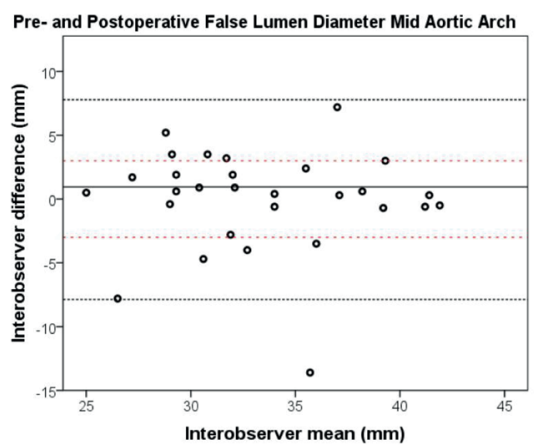

B

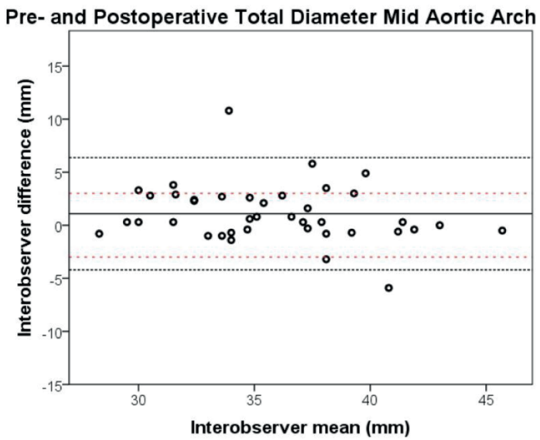

$\mathrm{D}$

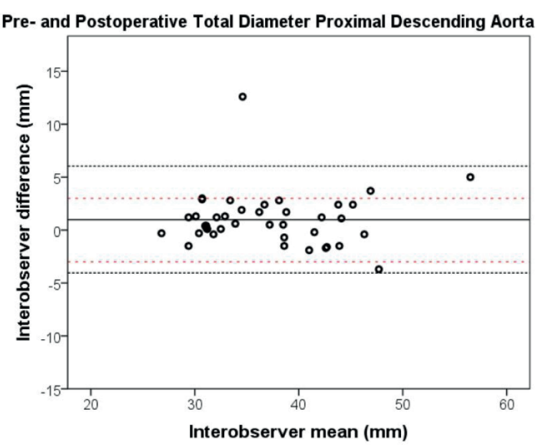

Interobserver Bland-Altman plots for maximum diameter measurements at the A) postrepair midascending aorta as a low-error reference for comparison, B) pre- and postoperative mid aortic arch, C) pre- and postoperative false luminal mid aortic arch and D) pre- and postoperative proximal descending aorta. The solid black line represents the mean of all measurements paired. The black close dotted lines represent the limits of agreement and the red double spaced dotted line represents the a priori defined acceptable differences. The limits of agreement are defined within range of the a priori acceptable differences when the black dotted lines both do not exceed the red dotted lines. 
Criticalappraisal ofmultidimensionalCTmeasurementsfollowingacuteopen repairoftypeAaorticdissection

Table $\mathbf{2}$ Interobserver agreement for preoperative mid-ascending aorta measurements. Continuous data are presented as the means \pm standard deviation.

\begin{tabular}{lcccc}
\hline & Mean \pm SD & $\begin{array}{c}\text { 95\% limits of } \\
\text { agreement }\end{array}$ & CCC & CCC agreement \\
\hline Total diameter $(\mathbf{m m})$ & $1.54 \pm 2.04$ & $-2.45 ; 5.53$ & $0.903(0.785 ; 0.958)$ & Moderate \\
\hline TL max diameter $(\mathbf{m m})$ & $0.31 \pm 1.92$ & $-3.45 ; 4.06$ & $0.957(0.897 ; 0.983)$ & Substantial \\
\hline FL max diameter $(\mathbf{m m})$ & $1.59 \pm 2.06$ & $-2.43 ; 5.62$ & $0.893(0.762 ; 0.954)$ & Fair \\
\hline Total area $(\mathbf{c m})$ & $1.03 \pm 0.89$ & $-0.71 ; 2.77$ & $0.953(0.899 ; 0.979)$ & Substantial \\
TL area $(\mathbf{c m})$ & $0.15 \pm 0.79$ & $-1.41 ; 1.70$ & $0.960(0.903 ; 0.984)$ & Substantial \\
FL area $(\mathbf{c m})$ & $0.88 \pm 0.92$ & $-0.92 ; 2.68$ & $0.955(0.900 ; 0.980)$ & Substantial \\
\hline
\end{tabular}

$\mathrm{CCC}=$ Concordance Correlation Coefficient, $\mathrm{FL}=$ False Lumen, $\mathrm{TL}=$ True Lumen

For total diameter measurements in the proximal descending aorta, the SD of the mean difference was $2.35 \mathrm{~mm}$ and limits of agreement were exceeding the a priori acceptable differences (Figure 3D). In all locations in the descending aorta, diameter measurements revealed a lower agreement score compared to area measurements (Tables 3). The distal descending thoracic aorta showed the lowest mean difference and lowest variability for diameter and area assessment (Table 3).

\section{Ellipticity}

The average ellipticity index at the six locations for TL and FL were defined (Table 4). The index values were higher than 2 in almost all regions of the aorta, suggesting no circularity for both the TL and the FL. The highest ellipticity index with the largest SD was present in the aortic arch FL; $4.76 \pm 4.97$. No correlation was found between variability of the measurements and ellipticity $(p=0.225)$. However, a significant negative correlation was found between ellipticity and interobserver agreement $(R=-0.693, p=$ $0.026)$, suggesting that it is more difficult to measure a non-circular aortic geometry.

\section{Volumetric agreement}

Volumetric assessment yielded a moderate agreement score for total, TL and FL volume of the pre- and postoperative CT measurements, with a CCC ranging 0.908-0.941. The mean difference of the total volumetric measurement was $19.20 \pm 14.52 \mathrm{~mL}$ with $95 \%$ limits of agreement of -9.26 to $47.66 \mathrm{~mL}$. The $\mathrm{FL}$ volume showed similar variability and $95 \%$ limits of agreement of -17.77 to $38.43 \mathrm{~mL}$. The variability was lower in the TL volume measurements with a mean difference of $9.07 \pm 4.79 \mathrm{~mL}$.

\section{DISCUSSION}

Morphologic CTA measurements are the main source of information for the assessment of aortic remodeling following acute TAAD. Measurement errors may have an important 
Current status of type A aortic dissection management

Table $\mathbf{3}$ Interobserver agreement for pre-and postoperative aortic arch up to infrarenal aortic measurements. Continuous data are presented as the means \pm standard deviation.

\begin{tabular}{|c|c|c|c|c|}
\hline Mid aortic arch & Mean \pm SD & $95 \%$ limits of agreement & $\mathrm{CCC}$ & CCC agreement \\
\hline Total diameter (mm) & $1.09 \pm 2.58$ & $-3.97 ; 6.16$ & $0.786(0.563 ; 0.903)$ & Poor \\
\hline TL max diameter (mm) & $-0.49 \pm 2.98$ & $-6.32 ; 5.35$ & $0.714(0.433 ; 0.870)$ & Poor \\
\hline FL max diameter (mm) & $-0.69 \pm 4.10$ & $-8.63 ; 7.25$ & $0.626(0.207 ; 0.865)$ & Poor \\
\hline Total area $(\mathrm{cm} 2)$ & $0.45 \pm 1.29$ & $-2.08 ; 2.98$ & $0.775(0.543 ; 0.899)$ & Poor \\
\hline TL area $(\mathrm{cm} 2)$ & $0.02 \pm 0.80$ & $-1.55 ; 1.58$ & $0.864(0.701 ; 0.942)$ & Fair \\
\hline FL area $(\mathrm{cm} 2)$ & $0.43 \pm 1.38$ & $-2.28 ; 3.14$ & $0.742(0.684 ; 0.877)$ & Poor \\
\hline Proximal descending aorta & Mean \pm SD & $95 \%$ limits of agreement & $\mathrm{CcC}$ & CCC agreement \\
\hline Total diameter (mm) & $0.99 \pm 2.35$ & $-3.62 ; 5.60$ & $0.918(0.812 ; 0.966)$ & Moderate \\
\hline TL max diameter (mm) & $-0.05 \pm 1.76$ & $-3.51 ; 3.40$ & $0.911(0.791 ; 0.964)$ & Moderate \\
\hline FL max diameter (mm) & $0.17 \pm 2.45$ & $-4.64 ; 4.97$ & $0.904(0.776 ; 0.961)$ & Moderate \\
\hline Total area $(\mathrm{cm} 2)$ & $0.59 \pm 1.02$ & $-1.42 ; 2.59$ & $0.937(0.855 ; 0.974)$ & Moderate \\
\hline TL area $(\mathrm{cm} 2)$ & $0.03 \pm 0.49$ & $-0.93 ; 0.99$ & $0.956(0.896 ; 0.981)$ & Substantial \\
\hline FL area $(\mathrm{cm} 2)$ & $0.56 \pm 1.06$ & $-1.52 ; 2.63$ & $0.924(0.825 ; 0.986)$ & Moderate \\
\hline Mid descending aorta & Mean \pm SD & $95 \%$ limits of agreement & $\mathrm{CCC}$ & CCC agreement \\
\hline Total diameter (mm) & $0.97 \pm 1.77$ & $-2.51 ; 4.46$ & $0.882(0.737 ; 0949)$ & Fair \\
\hline TL max diameter (mm) & $-0.05 \pm 1.22$ & $-2.44 ; 2.34$ & $0.889(0.744 ; 0.954)$ & Fair \\
\hline FL max diameter (mm) & $1.02 \pm 2.32$ & $-3.52 ; 5.56$ & $0.840(0.660 ; 0.928)$ & Poor \\
\hline Total area $(\mathrm{cm} 2)$ & $0.45 \pm 0.57$ & $-0.67 ; 1.57$ & $0.928(0.842 ; 0.967)$ & Moderate \\
\hline TL area $(\mathrm{cm} 2)$ & $-0.17 \pm 0.26$ & $-0.53 ; 0.49$ & $0.984(0.961 ; 0.994)$ & Substantial \\
\hline FL area $(\mathrm{cm} 2)$ & $0.47 \pm 0.61$ & $-0.74 ; 1.67$ & $0.940(0.867 ; 0.974)$ & Moderate \\
\hline Distal descending aorta & Mean \pm SD & $95 \%$ limits of agreement & $\mathrm{ccC}$ & CCC agreement \\
\hline Total diameter (mm) & $0.66 \pm 0.88$ & $-1.07 ; 2.39$ & $0.923(0.829 ; 0.966)$ & Moderate \\
\hline TL max diameter (mm) & $-0.10 \pm 1.16$ & $-2.37 ; 2.17$ & $0.892(0.769 ; 0.951)$ & Fair \\
\hline FL max diameter (mm) & $0.66 \pm 1.18$ & $-1.65 ; 2.97$ & $0.874(0.724 ; 0.944)$ & Fair \\
\hline Total area $(\mathrm{cm} 2)$ & $0.34 \pm 0.36$ & $-0.36 ; 1.05$ & $0.909(0.808 ; 0.958)$ & Moderate \\
\hline TL area $(\mathrm{cm} 2)$ & $-0.05 \pm 0.35$ & $-0.73 ; 0.63$ & $0.923(0.823 ; 0.968)$ & Moderate \\
\hline FL area $(\mathrm{cm} 2)$ & $0.4 \pm 0.54$ & $-0.66 ; 1.45$ & $0.921(0.823 ; 0.966)$ & Moderate \\
\hline Infrarenal aorta & Mean \pm SD & $95 \%$ limits of agreement & $\mathrm{CcC}$ & CCC agreement \\
\hline Total diameter (mm) & $0.26 \pm 1.09$ & $-1.87 ; 2.39$ & $0.925(0.806 ; 0.972)$ & Moderate \\
\hline TL max diameter (mm) & $-0.49 \pm 1.88$ & $-4.17 ; 3.18$ & $0.886(0.703 ; 0.958)$ & Fair \\
\hline FL max diameter (mm) & $0.58 \pm 1.09$ & $-1.56 ; 2.71$ & $0.885(0.679 ; 0.962)$ & Fair \\
\hline Total area $(\mathrm{cm} 2)$ & $0.21 \pm 0.30$ & $-0.38 ; 0.79$ & $0.936(0.817 ; 0.978)$ & Moderate \\
\hline TL area $(\mathrm{cm} 2)$ & $-0.19 \pm 0.59$ & $-1.34 ; 0.97$ & $0.973(0.925 ; 0.990)$ & Substantial \\
\hline FL area $(\mathrm{cm} 2)$ & $0.25 \pm 0.33$ & $-0.40 ; 0.90$ & $0.936(0.826 ; 0.977)$ & Moderate \\
\hline
\end{tabular}

$\mathrm{CCC}=$ Concordance Correlation Coefficient, $\mathrm{FL}=$ False Lumen, $\mathrm{TL}=$ True Lumen

a) 8 missing patients because of missing subdiaphragmatic data 
Figure 4. Interobserver volumetric measurement Bland-Altman plots

A

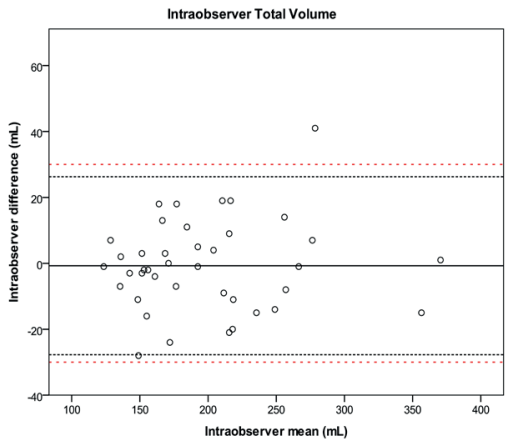

C

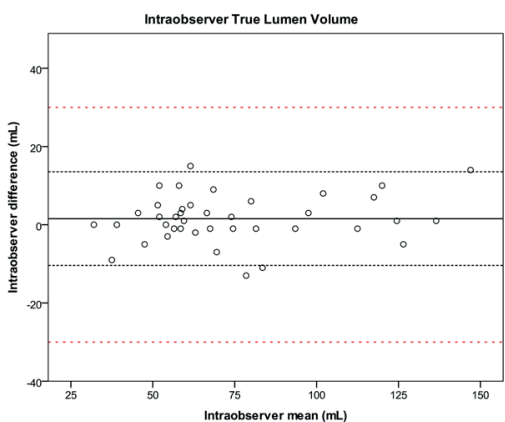

E

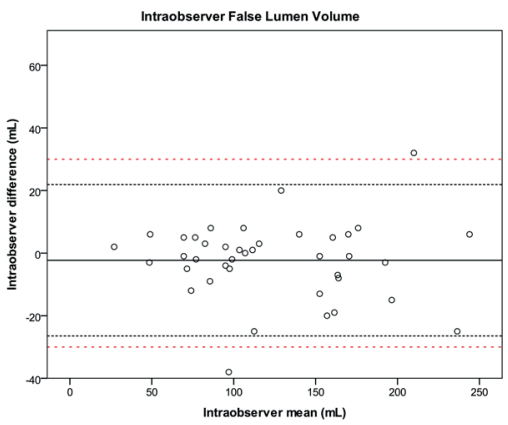

B

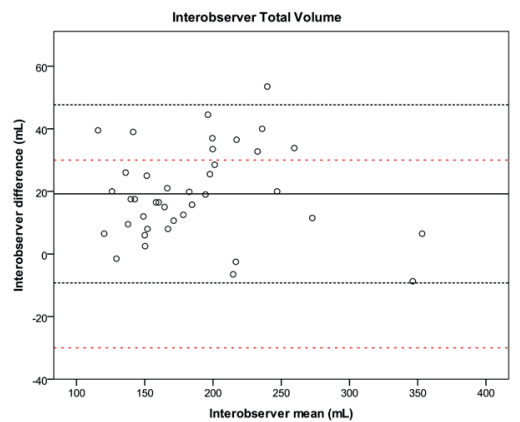

D

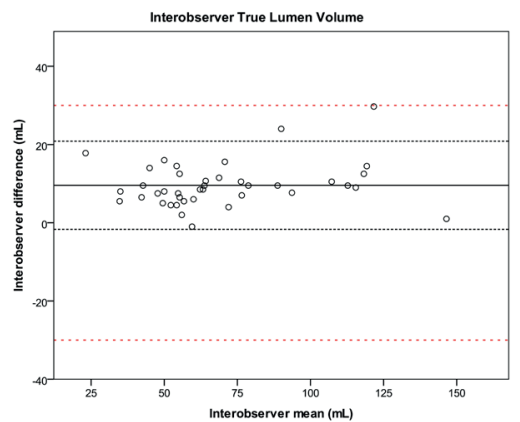

F

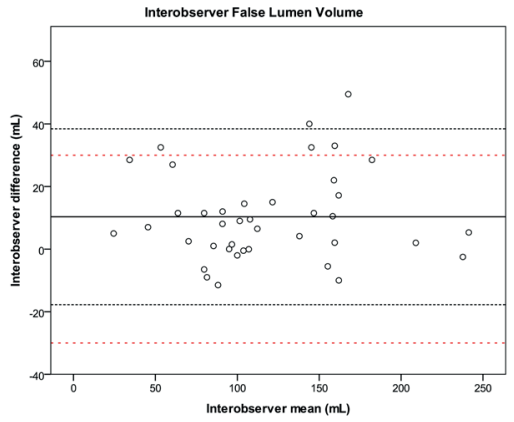

Intraobserver and interobserver Bland-Altman plots for preoperative and postoperative (A, B) total volumes, (C, D) true lumen volumes, and (E, F) false lumen volumes. The solid black line represents the mean of all measurements paired. The black close dotted lines represent the limits of agreement and the red double spaced dotted line represents the a priori defined acceptable differences. The limits of agreement are defined within the range of the a priori acceptable differences when the black dotted lines both do not exceed the red dotted lines. 
Table 4 Pre- and postoperative combined ellipticity for unrepaired measured aortic regions along with agreement scores and variability. Continuous data are presented as the means \pm standard deviation.

\begin{tabular}{lcccc}
\hline & Mean ellipticity & & & $\begin{array}{c}\text { Variability (average SD from } \\
\text { diameter measurements) }\end{array}$ \\
\hline Aortic arch & $\mathrm{TL}$ & $1.68 \pm 0.46$ & 0.761 & 3.81 \\
\hline Proximal desc & $\mathrm{FL}$ & $4.76 \pm 4.97$ & 0.594 & 4.92 \\
& $\mathrm{TL}$ & $2.18 \pm 1.50$ & 0.942 & 1.41 \\
\hline Mid descending & $\mathrm{FL}$ & $2.03 \pm 0.46$ & 0.880 & 4.08 \\
\hline Distal descending & $\mathrm{TL}$ & $2.69 \pm 1.62$ & 0.921 & 2.26 \\
\hline Infrarenal & $\mathrm{FL}$ & $2.05 \pm 1.44$ & 0.853 & 3.35 \\
\hline & $\mathrm{TL}$ & $2.65 \pm 1.54$ & 0.910 & 1.74 \\
\hline & $\mathrm{FL}$ & $1.92 \pm 0.64$ & 0.879 & 1.62 \\
\hline
\end{tabular}

$\mathrm{CCC}=$ concordance correlation coefficient, $\mathrm{FL}=$ false lumen, $\mathrm{TL}=$ true lumen

impact on patient-specific decision making. However, in the previously reported studies providing predicting factors for adverse aortic remodeling and aneurysmal formation, the reproducibility of various morphologic assessments was not assessed..$^{4,5,18-29}$ In the present study, we aimed to provide a comparison of the reproducibility of 2- and 3-dimensional morphologic measurement techniques.

We summarize our results as follows:

1. In postoperative TAAD patients, all luminal area measurements are more reproducible than luminal diameter measurements.

2. In these patients, measurement of the aortic arch shows lowest agreement in comparison to other aortic sites.

3. And volumetric measurements are not more reproducible than either regional diameter or area measurements.

To the best of our knowledge, this paper describes the first proposed systematic and validated aortic measurement workflow in early DeBakey type I aortic dissections. Our results show that, if a standardized workflow is used, total diameter, area and volumetric measurements of the thoracic aorta from CTA are reproducible. However, the separate luminal diameter measurements were less reproducible than luminal area measurements. This finding is of important clinical relevance for the assessment of growth in aortic dissections and therefore the timing of early intervention. Moreover, the sizing of stent-grafts in the case of early endovascular additional repair can be heavily misguided by simple diameter measurements, whereas area measurements may provide a more holistic approach, enabling adequate endovascular intervention. Given the variable 
morphology of the luminal dimensions in aortic dissection, Sailer et al. proposed to use the circumferential extent of the false lumen, ${ }^{30}$ reflecting the proportion of aortic wall circumference that is characterized by reduced thickness and strength. The study of Sailer et al. was performed in type B aortic dissection (TBAD) patients and has not yet been validated in other populations of aortic dissection, including post-repair TAAD patients. We did not include this method in this study, as we were interested in the most commonly performed clinical measurements described in predictor studies. The radial displacement of the aorta is known to be non-isometric throughout the cardiac cycle, however, routine clinical CTA data only contains static image data. ${ }^{31}$ Area measurements may account for these variations in deformations better than a single maximal or minimal diameter. We therefore elected to include area measurements. In order to assess the luminal differences in TAAD morphology, an ellipticity index was acquired. Rylski et al. defined circularity as an ellipticity index of $<1.1 .^{32}$ However, our results show that the $\mathrm{TL}$ and FL cannot be assumed to be circular in the early phase, since our lowest mean ellipticity index per region was 1.68 with a total range of $1.02-21.83$. We showed that area measurements have a higher agreement. We thus hypothesize that higher ellipticity index may correspond to less reproducible diameter measurements. Furthermore we expect the area measurements to be less affected by this loss in reproducibility. If area measurements are not feasible, then circumferential measurements should ideally be used.

The $C L$ proved useful for reliable diameter and area measurements. It is argued that a separate FL CL may provide more accurate FL assessment. ${ }^{15}$ The $\mathrm{CL}$ through the FL may however be harder for automated software to generate and clinical application could lead to comparison of an automated TL CL with a manual FL CL. This should in our opinion be avoided, as this will introduce an additional degree of variation to the analysis. Furthermore, the measurement of distance along the centerline will often disagree when using a separate $\mathrm{CL}$ for the TL and FL. For volumetric measurements, we did not use a manually generated $\mathrm{CL}$, but a semi-automated vessel filling tool. The borders of the volumes were set by anatomical landmarks (origin of the LSA and celiac trunk). This may account for the lower variability of the volume assessments as compared to the diameter and area assessments (Figure 2-4). Essentially, volumetric measurements provide more data points compared to two-dimensional diameter or area measurements. This may be particularly useful for assessment of eccentric aortic dilation during follow-up, although unlike luminal area or diameter measurements performed at specific levels, changes in volume do not reveal the location of growth. Although volumetric growth measurements demonstrated acceptable reproducibility, the agreement over time was no better than diameter or area measurements. 
Previous literature has reported lower variability of total volumetric and FL volumetric assessment in aneurysm and TBAD. ${ }^{15,33}$ In these reports the volumetric assessment was performed by manual or semi-automated delineation of the outer contour of the volumetric segment. Additionally, the volumetric assessment in aortic aneurysms shows lower variability, underlining the challenge of volumetric assessment in aortic dissection..$^{33}$ In our study we used a vessel growth tool that semi-automatically filled the vessel from the center of the lumen, which given that FL enhancement is often low-level and heterogeneous. This explains the high FL volumetric measurement variability compared to previous reports, as well as the acceptable measurement variability in the TL.

Early TEVAR is an evolving treatment strategy for type I dissections. The desired timeframe to assess early aortic growth allowing for sufficient aortic remodeling is well aligned with the timing of our measurements (i.e., 3 months post-operative). In our opinion, it is imperative to assess the reproducibility of a measurement paradigm that uses serial measurements.

\section{Limitations}

We have a relatively small sample size, however, there was appropriate statistical power based on our a priori calculations. We compared the pre-and postoperative CT scans and were not able to completely account for the effects of pre- and postoperative differences in FL contrast enhancement that could affect assessment of measurement variability. However, this scenario reflects the actual clinical challenge of determining FL growth at the earliest possible time point to allow for early intervention. Furthermore, it could be argued that we did not assess the association of FL growth with prior or subsequent events, although as we stated, performing a formal assessment of measurement technique was the focus of the paper and determining growth and outcomes was beyond the scope of this paper. A final limitation is arguably the human error introduced by manually segmenting the $\mathrm{CL}$, diameters, areas and volumetric measurements. In the near future, machine-learning will have the potential to improve software intelligence, in order to distinguish thrombus, low-flow lumen area, calcified plaque, aortic wall and surrounding tissue from one another.

We realize that there are more morphologic characteristics known in the literature to evaluate the aorta. In this study, we studied the most common clinically and scientifically used types and sites of aortic measurement. Comparing our outcomes to other described measurement protocols is beyond the scope of this study.

Entering the deep-learning age, we wish to stress that two-dimensional analysis limits accurate assessment. It seems suboptimal not to use all available imaging data, as most 
Criticalappraisal of multidimensionalCTmeasurementsfollowingacuteopen repairoftypeAaorticdissection

image processing software packages have three-dimensional tools which can provide important information if appropriately processed. The current study demonstrates that volumetric measurements yield acceptable intra- and interobserver variability, but perform worse than diameter and area measurements and need to be automated as much as possible in order to avoid human error and reduced reproducibility. Novel volumetric measurement techniques, such as Vascular Deformation Mapping ${ }^{34}$ may reduce the observer variability of aortic morphology over time, aiding identification of early adverse remodeling and selection of patients who would benefit from early TEVAR. Up till now this technique is not commonly used and should also be validated in a dissection population.

\section{CONCLUSION}

Commonly used methods of measuring aortic morphology were evaluated for intraand interobserver reproducibility, before and after open surgical repair of acute TAAD. Overall observer agreement is acceptable in total diameter, total area and volumetric measurements in early TAAD patients. TL and FL diameter measurements have a lower observer agreement, particularly in the aortic arch and the proximal descending aorta. In these locations, area measurements were more reproducible. While diameter measurements are most commonly used to assess aortic enlargement over time, the present study demonstrated that area measurements provide a more reproducible assessment of luminal morphology in patients with a TAAD. The addition of luminal area and possibly volumetric measurements to the standard diameter-based assessment of aortic dimensions in patients with TAAD may significantly improve the reproducibility of aortic growth measurements, and therefore alter clinical decision making in specific cases. 


\section{REFERENCES}

1. Ikeno Y, Yokawa K, Koda Y, et al. The fate of the downstream aorta after open aortic repair for acute DeBakey type I aortic dissection: total arch replacement with elephant trunk technique versus non-total arch replacement†. Eur J Cardio-Thoracic Surg. 2018;0:1-9.

2. Pape LA, Awais M, Woznicki EM, et al. Presentation, diagnosis, and outcomes of acute aortic dissection: 17-year trends from the international registry of acute aortic dissection. J Am Coll Cardiol. 2015;66:350-358.

3. Dohle D-S, El Beyrouti H, Brendel L, et al. Survival and reinterventions after isolated proximal aortic repair in acute type A aortic dissectiont. Interact Cardiovasc Thorac Surg. 2019;28:981-988.

4. Halstead JC, Meier M, Etz C, et al. The fate of the distal aorta after repair of acute type A aortic dissection. J Thorac Cardiovasc Surg. 2007;133:127-135.

5. Leontyev S, Haag F, Davierwala PM, et al. Postoperative Changes in the Distal Residual Aorta after Surgery for Acute Type A Aortic Dissection: Impact of False Lumen Patency and Size of Descending Aorta. Thorac Cardiovasc Surg. 2017;65:90-98.

6. Investigators VR. Mid-term outcomes and aortic remodelling after thoracic endovascular repair for acute, subacute, and chronic aortic dissection: the VIRTUE Registry. Eur J Vasc Endovasc Surg. 2014;48:363-371.

7. Peterss S, Ross JA, Elefteriades JA, et al. Changing Pathology of the Thoracic Aorta From Acute to Chronic Dissection. 2016;68:1054-1065.

8. van Bakel TMJ, Figueroa CA, van Herwaarden JA, et al. Challenges of Thoracic Endovascular Aortic Repair for Type B Aortic Dissection. J Endovasc Ther. 2018;25:578-580.

9. Houben IB, Patel HJ. Acute Type A Aortic Dissection: Managing More Than Just the Entry-Tear. Semin Thorac Cardiovasc Surg. 2019;31:122-128.

10. Stanley GA, Murphy EH, Knowles M, et al. Volumetric analysis of type B aortic dissections treated with thoracic endovascular aortic repair. J Vasc Surg. 2011;54:985-992.

11. Tolenaar JL, Kern JA, Jonker FH, et al. Predictors of false lumen thrombosis in type B aortic dissection treated with TEVAR. Ann Cardiothorac Surg. 2014;3:255-263.

12. Lavingia KS, Larion S, Ahanchi SS, et al. Volumetric analysis of the initial index computed tomography scan can predict the natural history of acute uncomplicated type B dissections. J Vasc Surg. 2014;62:893-899.

13. Chen S, Larion S, Ahanchi SS, et al. A novel anatomic severity grading score for acute Type B aortic dissections and correlation to aortic reinterventions after thoracic endovascular aortic repair. J Cardiothorac Surg. 2017;12:53.

14. Liao J. Sample Size Calculation for an Agreement Study. Pharm Stat. 2010;9:125-132.

15. Kamman A V., van Herwaarden JA, Orrico M, et al. Standardized Protocol to Analyze Computed Tomography Imaging of Type B Aortic Dissections. J Endovasc Ther. 2016;23:472-482.

16. Lin LI-K. A Concordance Correlation Coefficient to Evaluate Reproducibility. 1989;45:255.

17. Lin LI, MCBride G, Bland JM, et al. A Proposal for Strength-of-Agreement Criteria for Lin's Concordance Correlation Coefficient. 2005;45:307-310.

18. Rylski B, Hahn N, Beyersdorf F, et al. Fate of the dissected aortic arch after ascending replacement in type A aortic dissection. Eur J Cardio-thoracic Surg. 2017;51:1127-1134.

19. Kim J, Ro SK, Kim JB, et al. Remnant aortic remodelling in younger patients after acute type I aortic dissection surgery. Eur J Cardio-Thoracic Surg. 2017;52:150-155.

20. Tsai TT, Evangelista A, Nienaber CA, et al. Partial Thrombosis of the False Lumen in Patients with Acute Type B Aortic Dissection. 2007;357:349-359.

21. Regeer M V., Martina B, Versteegh MIM, et al. Prognostic implications of descending thoracic aorta dilation after surgery for aortic dissection. J Cardiovasc Comput Tomogr. 2017;11:1-7.

22. Song JM, Kim SD, Kim JH, et al. Long-Term Predictors of Descending Aorta Aneurysmal Change in Patients With Aortic Dissection. J Am Coll Cardiol. 2007;50:799-804.

23. Tolenaar JL, Van Keulen JW, Trimarchi S, et al. Number of entry tears is associated with aortic growth in type B dissections. Ann Thorac Surg. 2013;96:39-42. 
Criticalappraisal ofmultidimensionalCT measurementsfollowingacuteopen repair oftypeAaortic dissection

24. Kamman A V., Brunkwall J, Verhoeven EL, et al. Predictors of aortic growth in uncomplicated type B aortic dissection from the Acute Dissection Stent Grafting or Best Medical Treatment (ADSORB) database. J Vasc Surg. 2017;65:964-971.

25. Suzuki T, Asai T, Kinoshita T. Predictors for Late Reoperation After Surgical Repair of Acute Type A Aortic Dissection. Ann Thorac Surg. 2018;106:63-69.

26. Immer FF, Krähenbühl E, Hagen U, et al. Large area of the false lumen favors secondary dilatation of the aorta after acute type A aortic dissection. Circulation. 2005;112:249-253.

27. Immer FF, Hagen U, Berdat PA, et al. Risk factors for secondary dilatation of the aorta after acute type A aortic dissection. Eur J Cardio-thoracic Surg. 2005;27:654-657.

28. Zierer A, Voeller RK, Hill KE, et al. Aortic Enlargement and Late Reoperation After Repair of Acute Type A Aortic Dissection. Ann Thorac Surg. 2007;84:479-487.

29. Kim JB, Lee CH, Lee TY, et al. Descending aortic aneurysmal changes following surgery for acute DeBakey type I aortic dissection. Eur J Cardio-thoracic Surg. 2012;42:851-857.

30. Sailer AM, Van Kuijk SMJ, Nelemans PJ, et al. Computed Tomography Imaging Features in Acute Uncomplicated Stanford Type-B Aortic Dissection Predict Late Adverse Events. Circ Cardiovasc Imaging. 2017;10:1-11.

31. van Prehn J, Vincken KL, Sprinkhuizen SM, et al. Aortic Pulsatile Distention in Young Healthy Volunteers is Asymmetric: Analysis with ECG-gated MRI. Eur J Vasc Endovasc Surg. 2009;37:168-174.

32. Rylski B, Blanke P, Beyersdorf F, et al. How Does the Ascending Aorta Geometry Change When It Dissects? J Am Coll Cardiol. 2014;63:1311-1319.

33. van Prehn J, van der Wal MBA, Vincken K, et al. Intra- and Interobserver Variability of Aortic Aneurysm Volume Measurement With Fast CTA Postprocessing Software. 2008;15:504-510.

34. Burris NS, Hoff BA, Kazerooni EA, et al. Vascular Deformation Mapping (VDM) of Thoracic Aortic Enlargement in Aneurysmal Disease and Dissection. Tomography. 2017;3:163-173. 

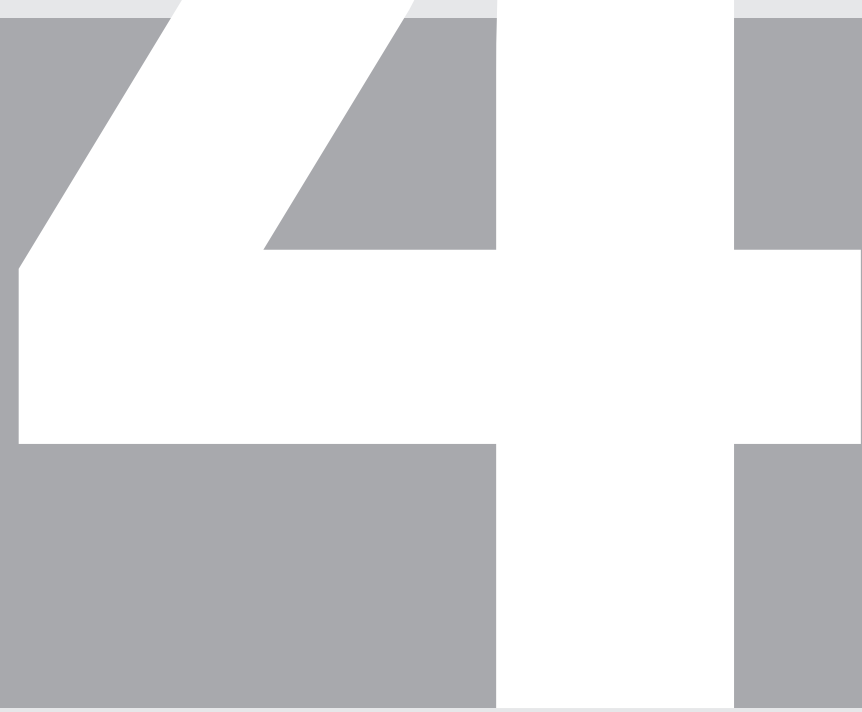


\section{Endovascular ascending aortic repair in type A aortic dissection: a systematic review}

Ignas B. Houben ${ }^{1,2}, \mathrm{MD}$

Yunus Ahmed ${ }^{1,2}$, MD,

C. Alberto Figueroa ${ }^{3}, \mathrm{PhD}$, Nicholas S. Burris ${ }^{4}, \mathrm{MD}$, David M. Williams ${ }^{4}, M D$, Frans L. Moll ${ }^{1}, \mathrm{MD}$, PhD Himanshu J. Patel' ${ }^{2}$, MD, Joost A. van Herwaarden ${ }^{1}, M D, P h D$

${ }^{1}$ Department of Vascular Surgery, Utrecht University, Utrecht, The Netherlands ${ }^{2}$ Department of Cardiac Surgery, Frankel Cardiovascular Center, University of Michigan, Ann Arbor, United States ${ }^{3}$ Departments of Surgery \& Biomedical Engineering, University of Michigan, Ann Arbor, United States ${ }^{4}$ Department of Radiology, University of Michigan, Ann Arbor, United States 


\section{ABSTRACT}

\section{Purpose}

Up to $15 \%$ of acute type A aortic dissection patients are deemed unfit for open surgical repair, exposing these patients to high mortality rates. In recent years, thoracic endovascular aortic repair has proven to be a promising and feasible alternative treatment modality in specific cases, although patient selection remains a topic of debate. This study presents a comprehensive overview of the current state of catheter-based interventions in the setting of primary type $\mathrm{A}$ aortic dissection.

\section{Methods}

A literature search was conducted, using MEDLINE and PubMed databases according to PRISMA guidelines, updated until January 2020. Two authors (Y.A., I.B.H.) independently assessed and analyzed the data. Articles were selected if they reported on endovascular repair of DeBakey type I and II aortic dissections. The exclusion criteria were as follows: retrograde type A dissection, hybrid procedures, combined outcome reporting of mixed aortic pathologies (e.g. (pseudo)aneurysm, intramural hematoma).

\section{Results}

A total of 31 articles, of which 19 were case reports and 12 case series, describing a total of 92 patients, were included. Median follow up was 6 for case reports and average follow up was 14 months for case series. Overall technical success was $95.6 \%$ and 30-day mortality of $9 \%$. Stroke and early endoleak rates were $6 \%$ and $18 \%$, respectively. Two patients $(2 \%)$ required intraoperative conversion to open surgery. Reintervention was required in 14 patients (15\%), of which four patients underwent endovascular repair and five patients underwent open repair.

\section{Conclusion}

This review not only demonstrates that endovascular repair in the setting of isolated type A aortic dissection is feasible with acceptable outcomes at mid-term follow up, but also underlines a lack of mid-late outcomes and reporting consistency. Studies with longer follow up and careful consideration of patient selection are required before endovascular interventions can be widely introduced as an alternative to the current gold standard. 


\section{INTRODUCTION}

Type A aortic dissection (TAAD) is a complex and challenging disease with morbidity and surgical mortality as high as $20 \% .{ }^{1-3}$ In the acute phase patients are exposed to an increasing mortality risk, estimated to be $1 \%$ each hour after onset of symptoms, and up to $90 \%$ 30-day mortality for medically treated acute TAAD patients. ${ }^{2,4}$ Considering the life-threatening course of this pathology, emergent intervention is paramount. Open surgical intervention is considered to be the gold standard in contemporary treatment, with acceptable outcomes. 5,6

In recent years, thoracic endovascular aortic repair (TEVAR) has been established as a viable treatment modality for various descending thoracic aortic pathologies with favorable outcomes..$^{7-9}$ Moreover, use of TEVAR has gradually progressed to the aortic arch and even ascending aorta, making it one of the last frontiers of endovascular aortic therapy. When considering patients who present with an acute TAAD, approximately $10 \%$ are deemed unfit for open surgical intervention, due to high and/or prohibitive risk. ${ }^{2,4}$ Recent studies report widely varying percentages (2-79\% percentage) of inoperable patients with an anatomy amenable for catheter-based interventions. ${ }^{10,11}$ Since the first TAAD endovascular repair, performed in 2000, endovascular therapy has seen rapid technological advancements in both device design and the capabilities of imaging technology used to assess endovascular candidacy and plan intervention. ${ }^{12}$ As such, endovascular aortic repair has emerged as a feasible and potentially suitable alternative for patients who are deemed unfit to undergo surgical intervention. Endovascular repair of TAAD is an understudied field, presenting novel challenges in device and delivery system design, and the endovascular procedure itself. In recent years various studies reported on endovascular repair of aortic arch and ascending aortic disease. ${ }^{13-19}$ However, there are distinct differences in anatomy and complexity of disease when dealing with a dissected aorta. Compared to conditions such as aneurysms, intramural hematoma (IMH) or penetrating atherosclerotic ulcer (PAU), dissection is characterized by loss of structural wall integrity through delamination and complicates identification of stable landing zones. ${ }^{19}$ Therefore, we aim to present a comprehensive literature overview of contemporary results of TEVAR, exclusively in the setting of TAAD with an entry tear in the ascending aorta.

\section{METHODS}

A thorough search of the MEDLINE and PubMed database was conducted, updated until January 2020. The used search terms were: 'Thoracic Endovascular Aortic Repair', 'Type A Dissection' and 'Ascending Aorta', including all variations of these terms. Relevant articles retrieved from the reference lists were subsequently added. The search was 
conducted according to the Preferred Reporting Items for Systematic Reviews and MetaAnalyses (PRISMA) guidelines. ${ }^{20}$ Two authors (Y.A., I.B.H) independently extracted and assessed all data for eligibility and reached consensus on the final selection. Articles were included if they reported on endovascular intervention of TAAD, with the entry tear located in the ascending aorta (i.e. DeBakey classification I and II). Exclusion criteria were: retrograde TAAD and hybrid procedures. Supra-aortic arch revascularization and debranching procedures prior to endovascular repair of TAAD, were not excluded. Furthermore, articles reporting on combined aortic pathology in the ascending aorta (e.g. thoracic aortic aneurysm [TAA]/pseudoaneurysm, IMH or PAU) that did not explicitly report on TAAD outcomes were excluded.

\section{RESULTS}

The initial search yielded a total of 805 articles, of which 736 were excluded based on title and/or abstract. The remaining 69 articles were thoroughly reviewed for eligibility, in addition to 9 articles that were extracted from selected articles reference lists. An additional 47 articles were excluded owing to description of pathology other than ascending aortic dissection, intervention other than endovascular ascending aortic repair with stent graft or inadequate or duplicate reporting of outcomes. At the completion of review, 31 studies were included (19 case reports and 12 case series) describing a total of 92 patients who underwent ascending aortic endovascular repair for TAAD (Figure 1) .${ }^{12,21,30-39,22,40-49,23,50,24-29}$ The selected studies were published between 2000 to 2019.

Figure 1. Flowchart of the study selection process

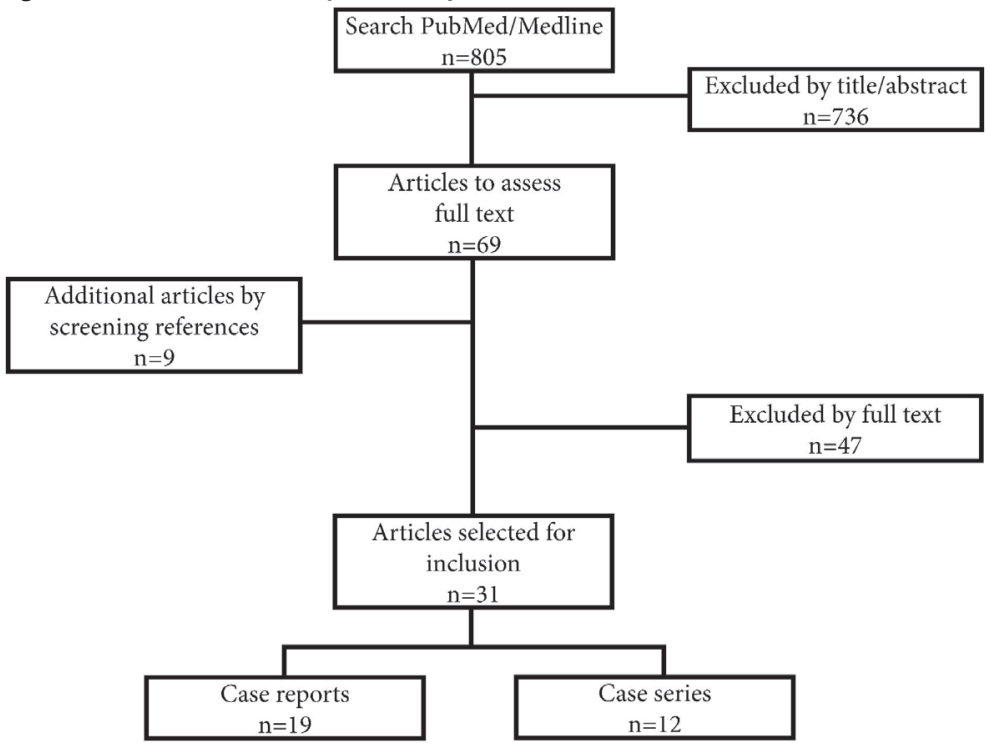


Patients presented with acute $(n=55)$, subacute $(n=10)$, or chronic TAAD $(n=27)$, defined as 0-14 days, 15-90 days and >90 days after onset of symptoms, respectively. Preoperative demographics are reported in Table 1 and Table 2 for the case reports and case series, respectively. Heterogeneity in reported literature precluded any meta-analysis of the data.

\section{Operative management and technique}

Use of anesthesia was described in 23 studies, with $98 \%$ of cases performed under general anesthesia. Two main methods of blood pressure management during stent graft deployment exist; rapid ventricular pacing (77\%) and medicine induced cardiac arrest or hypotension (22\%). Alternatively, one patient received venous inflow occlusion and in one patient a TandemHeart (CardiacAssist, Inc., Pittsburgh, PA, USA) was applied in addition to rapid ventricular pacing (Table 3 and 4). Moreover, in $75 \%$ of cases, the procedure was performed under trans esophageal echocardiogram (TEE) guidance. The endografts used in each study are reported in Tables 3 and 4 . Out of all 31 studies, transfemoral access as a device delivery route was reported in 72 patients $(78 \%$, Table 3 and 4$)$. Other types of vascular access were transapical $(n=14,15 \%)$, transcarotid $(n=2$, $2 \%$ ) or transaxillary ( $\mathrm{n}=4,4 \%)$. Insufficient additional specifics were reported regarding mode of access (i.e. cut-down or percutaneous). Overall technical success was $95.6 \%$.

\section{Hospital stay and follow-up}

Hospital stay was described in 11 case reports with a median of 5 (range 1.5 - 12) days. In only two out of 12 case series hospital stay was described, averaging 8.7 days over a total of 23 patients. Follow-up was described in 10 case reports with a median of 6 months (range 1 - 25). In the case series, follow-up was described in 7 studies (32 patients), averaging 14 months (range 18 - 39 months).

\section{Complications}

Early endoleak occurred in 17 out of 92 patients (18\%), and 4 patients required additional management using stent graft extensions. ${ }^{31,43,45}$ One patient received an Amplatzer plug to treat type IA endoleak. ${ }^{28}$ This was achieved through transapical access, after which the plug was used to eliminate residual flow across the entry tear at the proximal aspect of the stent graft. Late endoleak occurred in 9 patients (10\%). Most patients with endoleak developed no major complications, other than additional endovascular procedures. Subdividing the occurrence of endoleaks into proximal and distal leaks was impossible, as the location was not explicitly reported in all studies. However, most of the bird-beak signs (i.e. wedge-shaped gap between endograft and aortic wall due to incomplete apposition of the proximal portion of the endograft) were seen in the distal landing zone, as can be expected from its curved geometry. ${ }^{51}$ 


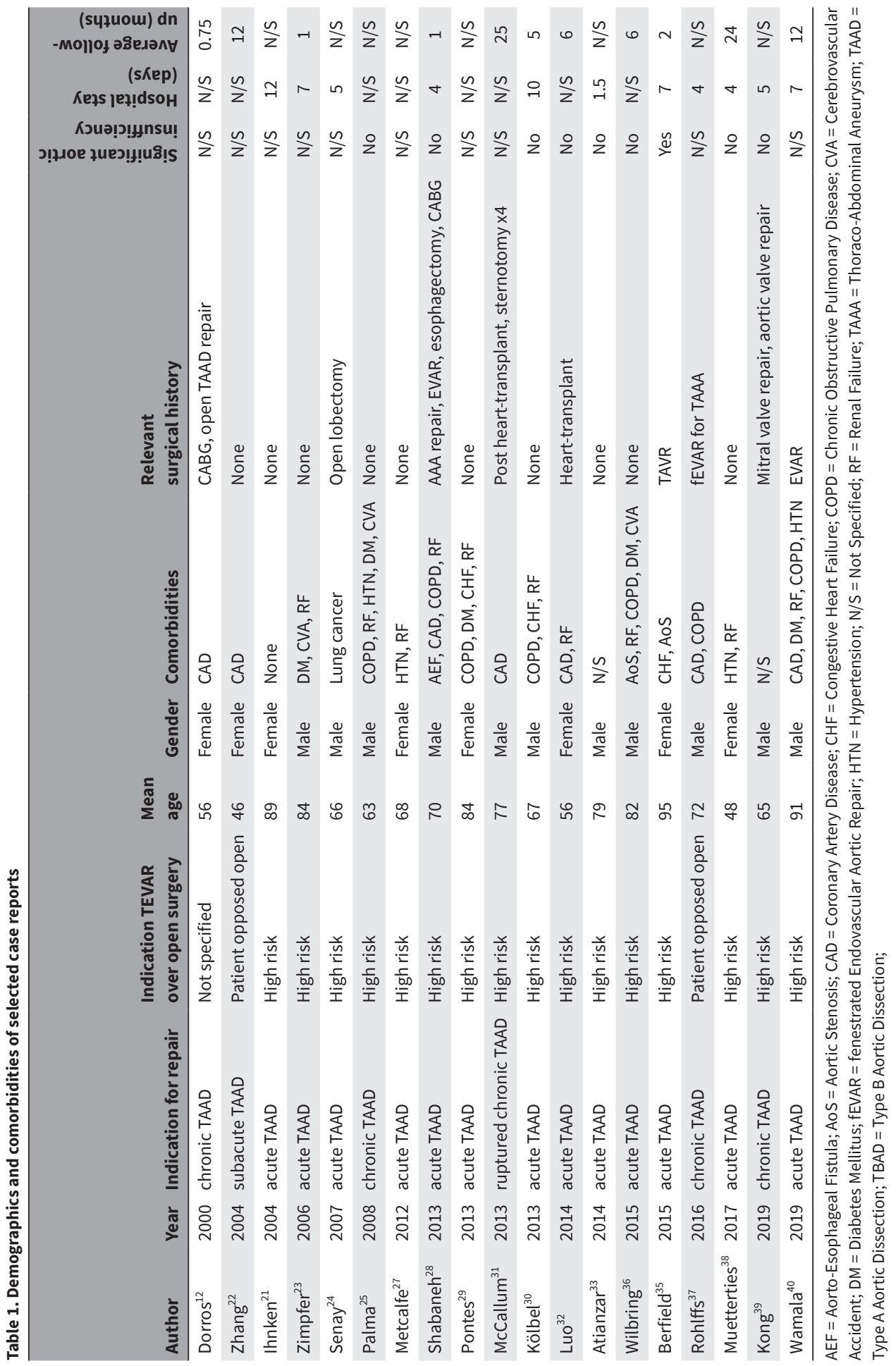


Endovascular ascending aortic repair in type A aortic dissection: a systematic review.

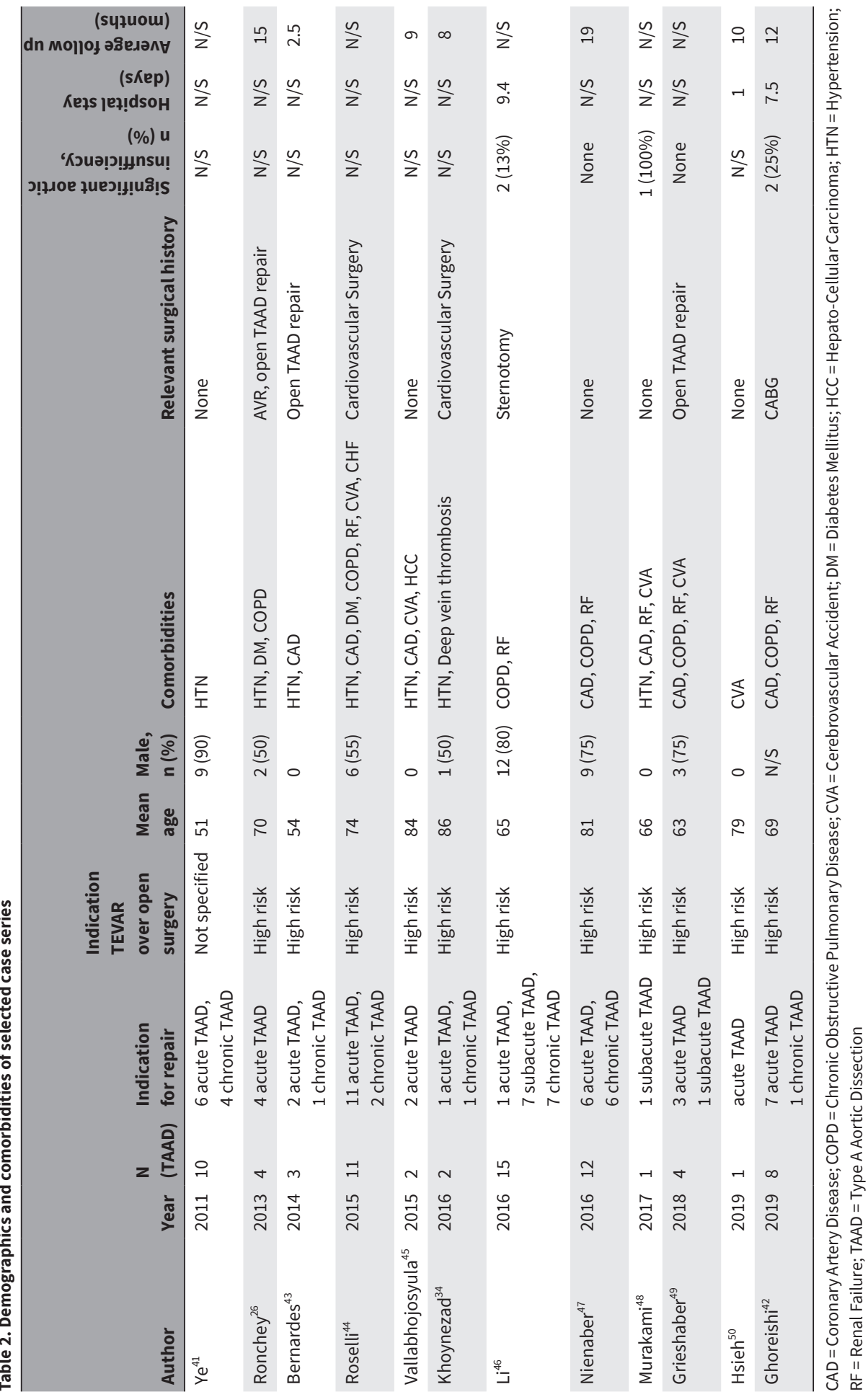




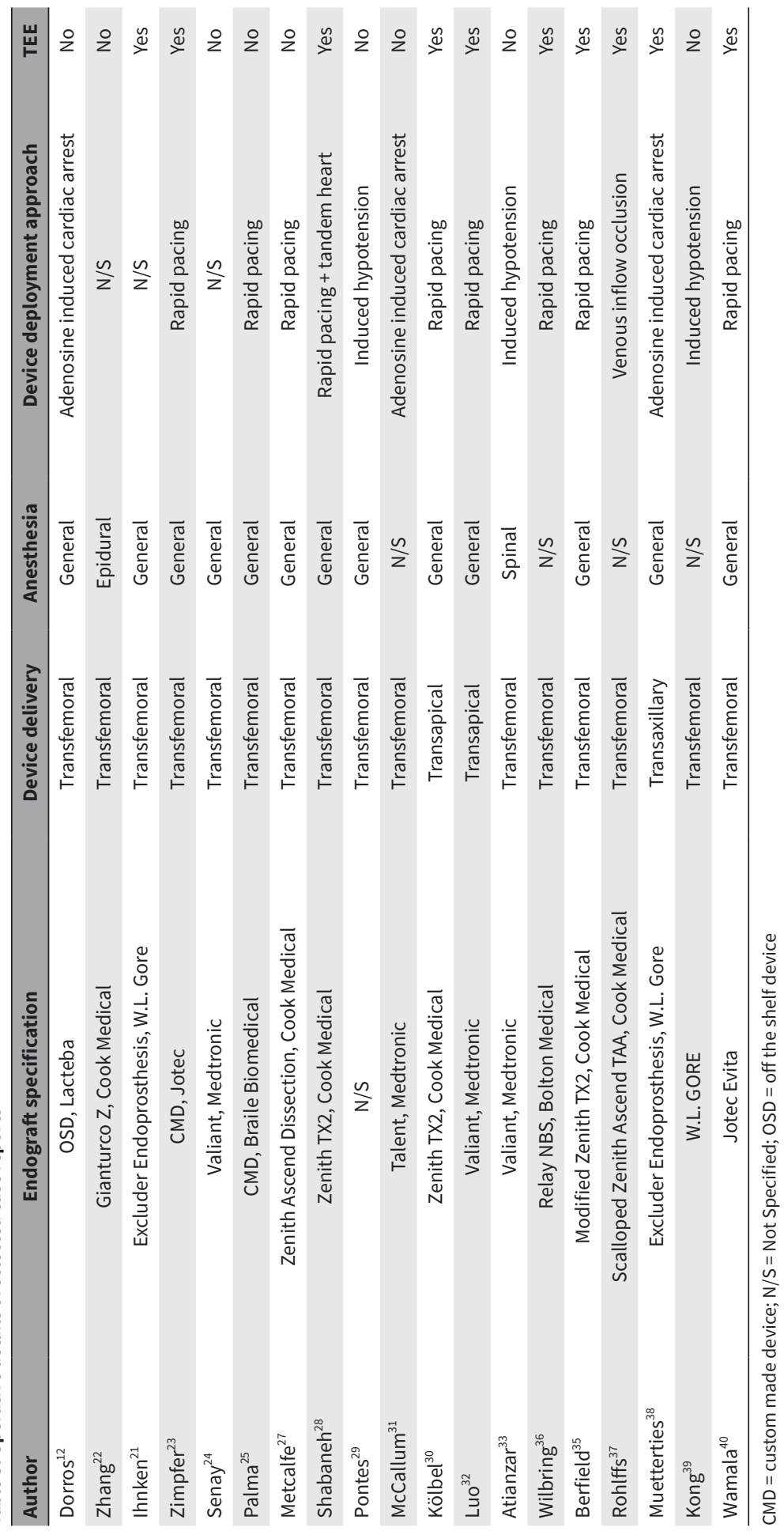




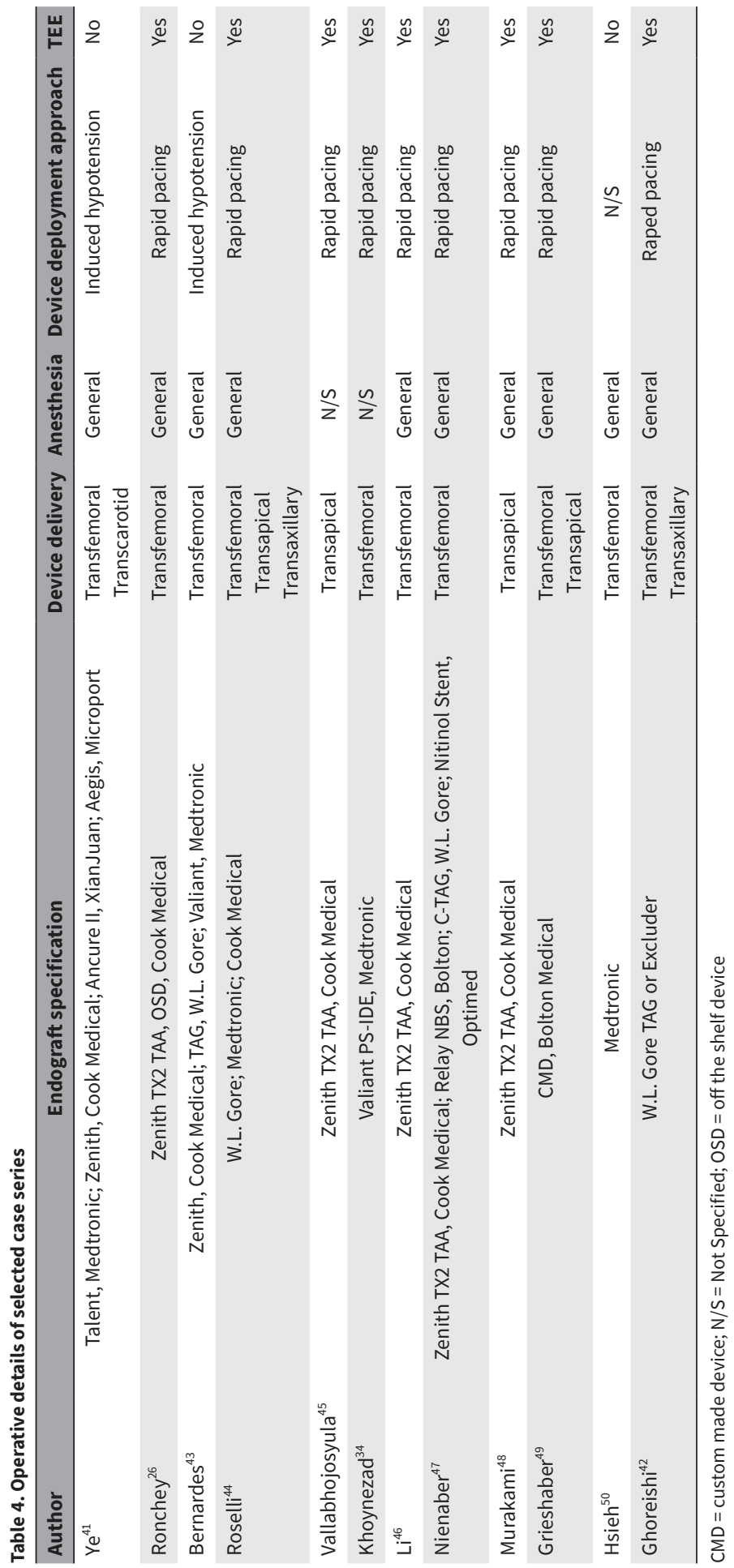


Current status of type A aortic dissection management

Table 5. In-hospital and late outcomes of selected case reports and case series

\begin{tabular}{lcc} 
& Case reports & Case series \\
\cline { 2 - 3 } & No. of patients (\%) & No. of patients (\%) \\
\hline Early outcomes & Total $n=19$ & Total $n=73$ \\
Technical success & $19(100)$ & $69(95)$ \\
Endoleak & $4(21)$ & $13(18)$ \\
Stroke & $1(5)$ & $5(7)$ \\
Myocardial infarction & $1(5)$ & $1(1)$ \\
Respiratory failure requiring prolonged ventilation or intubation & $1(5)$ & $0(0)$ \\
Renal failure requiring dialysis & $0(0)$ & $1(1)$ \\
Aortic insufficiency & $1(5)$ & $1(1)$ \\
30-day mortality & $2(11)$ & $6(8)$ \\
Late outcomes & Total $n=17$ & Total $n=67$ \\
Endoleak & $1(6)$ & $5(7)$ \\
Late mortality & 0 & $11(16)$ \\
\hline Stent-graft induced new-entry tears & 0 & $3(4)$
\end{tabular}

Rare complications included stent graft induced new entry-tears ( $n=2)$, accidental covering of branch vessels $(n=1)$, iatrogenic dissection $(n=1)$, or retained delivery systems $(n=1)$, and required immediate additional intervention in all cases, oftentimes including conversion to open repair. ${ }^{21,43,44}$

Overall, 30-day mortality was $9 \%$ (Table 5), with breakdowns of $11 \%, 0 \%$ and $4 \%$ for acute, subacute and chronic type A dissection, respectively. Late mortality was $16 \%$. Stroke rate was $6 \%$ (6 out of 92 ).

\section{Additional procedures}

Eight patients (9\%) required arch vessel debranching prior to TEVAR, while intraoperative aortic arch branching procedures were performed in 6 cases (7\%) (Table 6), using a chimney, fenestration or branched stent graft. Additional stenting or ballooning for endoleak or aortic valve entrapment was performed in 6 cases (7\%). Two patients (2\%) required direct open conversion.

\section{Reinterventions}

Ten patients (11\%) underwent open reintervention, whereas four patients (4\%) underwent endovascular reintervention (Table 6). Open reinterventions were performed for stent-graft induced new-entry tears (SINE) $(n=3)^{43,46}$, type I endoleak $(n=1)^{44}$, severe AI $(n=1)^{22}$, PAU $(n=1)^{34}$, iatrogenic femoral artery dissection $(n=1)^{42}$, pseudoaneurysm $(n=2)^{41,44}$ and pericardial effusion $(n=1)^{42}$. Endovascular reinterventions were performed for type I endoleak $(n=3)^{43,44}$ and severe $\mathrm{Al}(\mathrm{n}=1)^{42}$. 


\begin{tabular}{|c|c|c|c|}
\hline & & Case reports $(n=19)$ & Case series $(n=73)$ \\
\hline & & $\mathrm{n}(\%)$ & n (\%) \\
\hline \multirow{6}{*}{$\begin{array}{l}\text { Intraoperative } \\
\text { procedures }\end{array}$} & Arch vessel debranching & $0(0)$ & $8(11)$ \\
\hline & Branching procedures & $2(11)$ & $4(5)$ \\
\hline & Additional stenting & $1(5)$ & $3(4)$ \\
\hline & Additional ballooning & $1(5)$ & $1(1)$ \\
\hline & Open conversion & $0(0)$ & $2(3)$ \\
\hline & Coronary stenting & $1(5)$ & $1(1)$ \\
\hline \multirow[t]{2}{*}{ Reinterventions } & Open & $1(5)$ & $9(12)^{a}$ \\
\hline & Endovascular & $0(0)$ & $4(5)$ \\
\hline
\end{tabular}

${ }^{\mathrm{a}} 3$ out of 9 reinterventions were related to vascular access complications

\section{DISCUSSION}

Over recent years, endovascular interventions to treat type A aortic dissection have proven to be a feasible treatment modality. We report a 30 -day mortality of $9 \%$ and a mortality of $16 \%$ within 2 years. When compared to literature data on in-hospital mortality for open surgical repair of TAAD, ranging between 16 and 20\%, these results suggest that endovascular repair is an acceptable alternative for patients who are too frail to undergo open surgical repair for type A dissection. ${ }^{2,52}$ It is important to note that, similar to type $B$ aortic dissection, acuity of disease in TAAD impacts both patient presentation and outcome. ${ }^{53}$

Patients presenting with subacute/chronic TAAD have better short- and long-term survival rates when compared to acute TAAD patients. ${ }^{3}$ Rylski et al., confirmed lower inhospital mortality after surgery, although not significant, and better overall survival in chronic TAAD patients compared with acute TAAD patients. ${ }^{54}$ Given distinct differences in outcomes, we believe a careful analysis of these specific patient subsets based on acuity of disease is warranted. Unfortunately, the available data in current literature precluded this assessment. Stroke incidence with endovascular treatment of TAAD of $6 \%$ was no higher than previous reports from arch TEVAR ${ }^{55-57}$ and open TAAD repair. ${ }^{4,52,58}$ However, so far it is not possible to draw any meaningful conclusions without long-term outcomes.

TEVAR in the setting of TAAD is a novel and rapidly developing treatment modality with a reported acceptable incidence of complications compared to open surgical repair in reported cases. However, given the novelty of this procedure and the lack of long-term outcomes, there remain several topics of concern that must be adequately considered: patient selection, vascular access, stent graft design and aortic valve involvement. These topics will be addressed below. 


\section{Patient selection}

When considering endovascular repair in TAAD, rigorous patient selection is of paramount importance, based on both clinical and radiological characteristics. As of current, only patients who are considered too frail to undergo open surgical repair are considered for TEVAR. Patients with prohibitive fragility warrant a minimally-invasive approach and require careful planning. TAAD patients presenting with visceral malperfusion syndrome represent just such a population, with increased in-hospital mortality (71\%) compared to medically treated patients $(57 \%){ }^{2,59-61}$ Sustained positive outcomes using a treatment approach of initial endovascular malperfusion management (i.e. fenestration of the intimal flap and/or true lumen stenting), followed by delayed open repair have been demonstrated. ${ }^{62,63} \mathrm{~A}$ recent publication by Omura et al. demonstrates the feasibility of TEVAR and concomitant true lumen stenting in acute retrograde type A dissection with renal malperfusion. ${ }^{64}$ However, no cases of endovascular TAAD repair with concomitant endovascular visceral malperfusion treatment have been reported.

ECG-gated computed tomography (CT) scan technique is highly recommended given the requirement for motion-artifact free images for accurate measurements. Retrospective ECG-gating technique is optimal for quantification of morphometric changes of the aorta over the cardiac cycle, and for dynamic evaluation of the entry tear anatomy, particularly in acute/subacute TAAD given higher degrees of intimal flap motion ${ }^{65}$ As of recent, various studies have explored imaging characteristics in type A dissection patients to assess feasibility of endovascular aortic repair. ${ }^{10,11}$ Ideally, 2 centimeters of non-dilated and non-dissected aorta can be identified at both proximal and distal landing zones. Complex hemodynamics encountered in the thoraco-abdominal aorta leads to increased displacement forces on the stent graft and accentuate the need for a robust seal. ${ }^{66,67}$ Considering the limited length of the ascending aorta, any tears in close proximity to the innominate artery may allow for a modified approach to landing zone thresholds by employing a branched endograft or performing a cervical debranching procedure to allow coverage of arch vessel ostia by the endograft. In turn, the proximal landing zone poses a high risk of coronary occlusion and its downstream sequelae.

\section{Vascular access}

When treating proximal aortic disease, transfemoral delivery may be more limited as a result of inadequate delivery system length and higher degrees of aortic arch angulation. The iliofemoral arteries require a caliber of at least $6.5-7 \mathrm{~mm}$, as most conformable thoracic stents have a minimal profile of 20 French. ${ }^{68-71}$. Retrograde delivery routes carry an increased risk of aortic wall injury if true lumen access is not obtained at both landing zones. Wire location in the true lumen can be confirmed by IVUS examination before delivery of the endograft. Furthermore, accurate deployment may be complicated by 
increased spring-back forces or decreased conformability (i.e. increased rigidity) of the delivery device. ${ }^{72,73}$

Alternatively, a transapical, transcarotid or transaxillary approach offer deployment strategies closer to the ascending aorta when compared to the transfemoral approach. Nonetheless, each of these approaches carry distinct risks. Transapical delivery is incompatible with previous implanted prosthetic mechanical valves and may potentially induce long term complications, such as formation of left ventricular pseudoaneurysm, as reflected in this review. ${ }^{44,46}$ This approach however offers the unique advantage of antegrade deployment, ensuring true lumen access. Furthermore, the more in-line approach and shorter distance to the sinotubular junction (STJ) provides a lower dependence on delivery device conformability. ${ }^{11}$ Transcarotid and transaxillary access have been rarely described for proximal aortic TEVAR. In the two patient where transcarotid delivery was performed, stroke occurred within 3 weeks and 1 years postoperatively. ${ }^{41}$

\section{Stent graft design}

Most case reports describe stent grafts that were originally designed for the descending thoracoabdominal aorta. Consequently, these devices are often too long for use in the ascending aorta, frequently measuring over $10 \mathrm{~cm}$ in length, whereas the normal length of the ascending aorta is 8 to $10 \mathrm{~cm}^{74}$ Ideally, the endograft is long enough to span the entry tear with adequate landing zones while sparing the innominate and coronary arteries. Potential solutions include customizing existing descending stent-grafts or using commercially approved shorter segment devices. The first option comes with significant shortcomings, as the newly exposed cut-end of the endograft may cause damage to the adjacent aortic tissue and increase risk of SINE. Furthermore, unloading and reloading the device may lead to failure during deployment. The latter option has the advantage of accounting for the higher angulation through modular grafting and the preloaded market-approved devices. However, when using shorter segment stent grafts, there is often need to deploy multiple overlapping stents to ensure coverage of the ascending aorta, with a corresponding increased risk of type IIla endoleak (i.e. leak between endograft components) or migration. Metcalfe et al were the first to describe the use of a stent graft specifically designed for the ascending aorta, with excellent results. ${ }^{27}$

Stent graft design and deployment techniques need to account for the hemodynamic forces present in the ascending aorta, by ensuring adequate landing zone length and seal to withstand the displacement forces and ultimately prevent stent migration. ${ }^{75-77}$ A computational fluid dynamics study by Figueroa et al. evaluated net thoracic aortic stent graft displacement, demonstrating that the more proximal the endograft is implanted, the greater the cranial component of displacement. ${ }^{78}$ Furthermore, multiple 
studies have identified other factors contributing to displacement forces acting on both abdominal and thoracic aortic endografts including forces exerted by the blood flow, stent graft diameter and deployment position of the stent graft. ${ }^{66,67,79}$

In conventional endovascular aneurysm repair outer-wall diameter is used for stent graft sizing and $10-20 \%$ oversizing is generally accepted..$^{80}$ However, in dissection and IMH the size of the true lumen relative to the outer-wall diameter may be discrepant, difficult to measure and variable over time. Additionally, the tissue strength of the dissected segment may be unable to bear the radial force imposed by the endograft. To this extent, tissue fragility in the dissected ascending aorta must be taken into account, and stent graft oversizing of $10-20 \%$ may be excessive, potentially causing further wall injury or rupture. Predominantly in acute dissections, oversizing of $5-10 \%$ is advocated, whereas the amount of oversizing in chronic dissections may be higher. ${ }^{81-83}$ These oversizing rates have been based on TEVAR for descending thoracic aortic pathology, as limited ascending endovascular repair has not yet been described.

\section{Aortic valve involvement}

A major limitation of endovascular approach of type A repair is the limited ability to address aortic valve insufficiency (AI), either secondary to STJ malalignment or valve injury due to a proximal entry tear. In open repair for TAAD, Al can be treated using a valve resuspension technique or complete aortic valve replacement. As suggested previously by Kreibich et al. and more recently demonstrated by Gaia et al., an "endo-Bentall" approach might allow for treatment of $A l$ in the setting of TAAD. ${ }^{84,85}$ The authors describe a transapical approach, in which a TAVR valve is deployed simultaneously with an ascending aortic stent graft, successfully extending the proximal landing zone of the stent graft to the aortic annulus. The valve is connected to an uncovered portion of the stent graft, to allow for free diastolic coronary blood flow. In the case of an entry tear at the level of the sinus of Valsalva or lower, however, this approach may not fully exclude the entry tear, the primary goal of any TAAD repair technique. Currently, a proven endovascular solution to address significant $\mathrm{Al}$ is not available.

\section{Limitations}

This systematic review is limited by the quality of the existing literature, characterized by an overall lack of large cohort studies and prospective trials. It is therefore important to recognize the potential for publication bias in the studies reviewed in this paper, warranting caution in interpreting these early results. Further, we recognize the distinct differences in outcomes between patients presenting with acute and chronic TAAD, and the lack of a sub analysis for these cohorts is dissatisfying. Therefore, current results may not be fully generalizable to the entire TAAD population. Open repair for TAAD is still 
recognized as the gold standard and endovascular repair as a treatment modality still under development. Patients undergoing endovascular aortic repair represent a small subpopulation of all type A dissection patients, and thus most conclusions drawn from the current reports of endovascular TAAD repair are likely premature. In order to fully ascertain the role of TEVAR in the treatment of TAAD, larger studies with longer term follow-up are clearly needed. Given the heterogeneity and the small sample size of all reports, we were unable to apply more advanced statistical techniques (e.g., randomeffects meta-analysis model), and are thus limited to descriptive and pooled analyses.

\section{Future perspective}

In the past decade, we have seen an increasing number of reports advocating for and demonstrating feasibility of endovascular alternatives for patients with high risk for surgical repair of type A dissection. Given the estimated incidence of acute type A dissection of 3-5 per 100,000 patients and the observation that approximately $9-14 \%$ of patients are deemed inoperable, one would expect a broad application of such novel endovascular techniques. However, in the past two decades we have identified a total of only 92 type A dissection patients managed with TEVAR despite a comprehensive literature review. Remarkably, and quite possibly due to underreporting of poor outcomes, the overall mortality and complication rate in these patients appears comparable to gold standard open repair. ${ }^{2,52}$ To unequivocally test the validity of such novel endovascular techniques, randomized controlled trials will be required. However, given the long-standing paradigm of open repair for treatment of type A dissection, such trials have been deemed unfeasible. Lastly, to advance knowledge and provide a more comprehensive understanding of treatment effectiveness, we advocate for reporting all outcomes, both positive and negative, in order to avoid publication bias and to confirm existing reports demonstrating comparable outcomes between open and endovascular treatment of type A dissection.

\section{CONCLUSION}

This systematic analysis presents a comprehensive review of isolated TEVAR management of type A dissection. Our review confirms that TEVAR is a feasible treatment option for high-risk TAAD patients and current reports show results that are favorable over medical treatment. These pooled results suggest that endovascular intervention for type A dissection is feasible with acceptable mid-term results. However, this review also highlights the urgent need for technical advances in disease-specific device design, delivery methods, and hybrid endovascular techniques to address the need for concomitant valve replacement and/or arch endografting. Most importantly, our analysis emphasizes the significant need for larger studies with long-term results and more complete reporting of negative results before the endovascular intervention may be widely proposed as an alternative to the gold standard open repair. 


\section{REFERENCES}

1. Conzelmann LO, Weigang E, Mehlhorn U, et al. Mortality in patients with acute aortic dissection type A: Analysis of pre- and intraoperative risk factors from the German Registry for Acute Aortic Dissection Type A (GERAADA). Eur J Cardio-thoracic Surg. 2016;49(2):e44-e52. doi:10.1093/ejcts/ezv356

2. Pape LA, Awais M, Woznicki EM, et al. Presentation, diagnosis, and outcomes of acute aortic dissection: 17year trends from the international registry of acute aortic dissection. J Am Coll Cardiol. 2015;66(4):350-358. doi:10.1016/j.jacc.2015.05.029

3. Wu J, Xie E, Qiu J, et al. Subacute/chronic type A aortic dissection: A retrospective cohort study. Eur J Cardiothoracic Surg. 2020;57(2):388-396. doi:10.1093/ejcts/ezz209

4. Berretta P, Patel HJ, Gleason TG, et al. IRAD experience on surgical type A acute dissection patients: Results and predictors of mortality. Ann Cardiothorac Surg. 2016;5(4):346-351. doi:10.21037/acs.2016.05.10

5. Hiratzka LF, Bakris GL, Beckman JA, et al. 2010 ACCF/AHA/AATS/ACR/ASA/SCA/SCAI/SIR/STS/SVM Guidelines for the Diagnosis and Management of Patients with Thoracic Aortic Disease. Anesth Analg. 2010;111(2):279-315. doi:10.1016/j.jacc.2010.02.010

6. Erbel R, Aboyans V, Boileau C, et al. 2014 ESC guidelines on the diagnosis and treatment of aortic diseases. Russ J Cardiol. 2015;123(7):7-72. doi:10.15829/1560-4071-2015-07-7-72

7. Abraha I, Romagnoli C, Montedori A, et al. Thoracic stent graft versus surgery for thoracic aneurysm. Cochrane Database Syst Rev. 2016;2016(6). doi:10.1002/14651858.CD006796.pub4

8. Coady MA, Ikonomidis JS, Cheung AT, et al. Surgical management of descending thoracic aortic disease: Open and endovascular approaches: A scientific statement from the American heart association. Circulation. 2010;121(25):2780-2804. doi:10.1161/CIR.0b013e3181e4d033

9. Cheng D, Martin J, Shennib H, et al. Endovascular Aortic Repair Versus Open Surgical Repair for Descending Thoracic Aortic Disease. A Systematic Review and Meta-Analysis of Comparative Studies. J Am Coll Cardiol. 2010;55(10):986-1001. doi:10.1016/j.jacc.2009.11.047

10. Nissen AP, Ocasio L, Tjaden BL, et al. VESS28. Imaging Characteristics of Acute Type A Aortic Dissection and Candidacy for Repair With Ascending Aortic Endografts. J Vasc Surg. 2019;69(6):e72-e73. doi:10.1016/j. jvs.2019.04.055

11. Roselli EE, Hasan SM, Idrees JJ, et al. Inoperable patients with acute type A dissection: Are they candidates for endovascular repair? Interact Cardiovasc Thorac Surg. 2017;25(4):582-588. doi:10.1093/icvts/ivx193

12. Dorros G, Dorros AM, Planton S, et al. Transseptal Guidewire Stabilization Facilitates Stent-Graft Deployment for Persistent Proximal Ascending Aortic Dissection. J Endovasc Ther. 2000;7:506-512.

13. Böckler D, Brunkwall J, Taylor PR, et al. Thoracic Endovascular Aortic Repair of Aortic Arch Pathologies with the Conformable Thoracic Aortic Graft: Early and 2 year Results from a European Multicentre Registry. Eur J Vasc Endovasc Surg. 2016;51(6):791-800. doi:10.1016/j.ejvs.2016.02.006

14. Shijo T, Kuratani T, Torikai K, et al. Thoracic endovascular aortic repair for degenerative distal arch aneurysm can be used as a standard procedure in high-risk patients. Eur J Cardio-thoracic Surg. 2016;50(2):257-263. doi:10.1093/ejcts/ezw020

15. Wang T, Shu C, Li M, et al. Thoracic Endovascular Aortic Repair with Single/Double Chimney Technique for Aortic Arch Pathologies. J Endovasc Ther. 2017;24(3):383-393. doi:10.1177/1526602817698702

16. Baikoussis NG, Antonopoulos CN, Papakonstantinou NA, et al. Endovascular stent grafting for ascending aorta diseases. J Vasc Surg. 2017;66(5):1587-1601. doi:10.1016/j.jvs.2017.07.064

17. Muetterties CE, Menon R, Wheatley GH. A systematic review of primary endovascular repair of the ascending aorta. J Vasc Surg. 2018;67(1):332-342. doi:10.1016/j.jvs.2017.06.099

18. Piffaretti G, Grassi V, Lomazzi C, et al. Thoracic endovascular stent graft repair for ascending aortic diseases. $J$ Vasc Surg. 2019;70(5):1384-1389.e1. doi:10.1016/j.jvs.2019.01.075

19. Kratimenos T, Baikoussis NG, Tomais D, et al. Ascending Aorta Endovascular Repair of a Symptomatic Penetrating Atherosclerotic Ulcer with a Custom-Made Endograft. Ann Vasc Surg. 2018;47:280.e1-280.e4. doi:10.1016/j. avsg.2017.08.027 
Endovascular ascending aortic repair in type A aortic dissection: a systematic review.

20. Liberati A, Altman DG, Tetzlaff J, et al. The PRISMA statement for reporting systematic reviews and metaanalyses of studies that evaluate health care interventions: explanation and elaboration. J Clin Epidemiol. 2009;62(10):e1-e34. doi:10.1016/j.jclinepi.2009.06.006

21. Ihnken K, Sze D, Dake MD, et al. Successful treatment of a Stanford type A dissection by percutaneous placement of a covered stent graft in the ascending aorta. J Thorac Cardiovasc Surg. 2004;127(6):1808-1810. doi:10.1016/j. jtcvs.2003.12.019

22. Zhang H, Li M, Jin W, et al. Endoluminal and surgical treatment for the management of Stanford Type A aortic dissection. Eur J Cardio-thoracic Surg. 2004;26(4):857-859. doi:10.1016/j.ejcts.2004.07.008

23. Zimpfer D, Czerny M, Kettenbach J, et al. Treatment of Acute Type A Dissection by Percutaneous Endovascular Stent-Graft Placement. Ann Thorac Surg. 2006;82(2):747-749. doi:10.1016/j.athoracsur.2005.11.066

24. Senay S, Alhan C, Toraman F, et al. Endovascular Stent-graft Treatment of Type A Dissection: Case Report and Review of Literature. Eur J Vasc Endovasc Surg. 2007;34(4):457-460. doi:10.1016/j.ejvs.2007.06.015

25. Palma JH. Endovascular treatment of chronic type A dissection. Interact Cardiovasc Thorac Surg. 2008;7(1):166166. doi:10.1510/icvts.2007.165027a

26. Ronchey S, Serrao E, Alberti V, et al. Endovascular stenting of the ascending aorta for type A aortic dissections in patients at high risk for open surgery. Eur J Vasc Endovasc Surg. 2013;45(5):475-480. doi:10.1016/j. ejvs.2013.01.033

27. Metcalfe MJ, Karthikesalingam A, Black SA, et al. The first endovascular repair of an acute type A dissection using an endograft designed for the ascending aorta. J Vasc Surg. 2012;55(1):220-222. doi:10.1016/j.jvs.2011.06.116

28. Shabaneh B, Gregoric ID, Loyalka P, et al. Complex endovascular repair of a large dissection of the ascending aorta in a 70-year-old man. Texas Hear Inst J. 2013;40(2):182-185.

29. Pontes JC, Dias AM, Duarte JJ, et al. Endovascular repair of ascending aortic dissection. Rev Bras Cir CardiovasC. 2013;28(1):145-147. doi:10.1053/j.semtcvs.2005.06.005

30. Kölbel T, Reiter B, Schirmer J, et al. Customized transapical thoracic endovascular repair for acute type A dissection. Ann Thorac Surg. 2013;95(2):694-696. doi:10.1016/j.athoracsur.2012.06.055

31. McCallum JC, Limmer KK, Perricone A, et al. Case report and review of the literatureTotal Endovascular Repair of Acute Ascending Aortic Rupture: A Case Report and Review of the Literature. Vasc Endovascular Surg. 2013;47(5):374-378. doi:10.1177/1538574413486838

32. Luo CM, Wang SS, Chi NH, et al. Transapical Endovascular Repair with a Table-Tailored Endograft to Treat Ascending Aortic Dissection. J Card Surg. 2014;29(6):824-826. doi:10.1111/jocs.12449

33. Atianzar K, Mohamad A, Galazka P, et al. Endovascular stent-graft repair of ascending aortic dissection with a commercially available thoracic endograft. Ann Thorac Surg. 2014;98(2):715-717. doi:10.1016/j.athoracsur.2013.09.047

34. Khoynezhad A, Donayre CE, Walot I, et al. Feasibility of endovascular repair of ascending aortic pathologies as part of an FDA-approved physician-sponsored investigational device exemption. J Vasc Surg. 2016;63(6):14831495. doi:10.1016/j.jvs.2015.12.029

35. Berfield KKS, Sweet MP, McCabe JM, et al. Endovascular repair for type A aortic dissection after transcatheter aortic valve replacement with a medtronic corevalve. Ann Thorac Surg. 2015;100(4):1444-1446. doi:10.1016/j. athoracsur.2014.11.077

36. Wilbring M, Ghazy T, Matschke K, et al. Complete endovascular treatment of acute proximal ascending aortic dissection and combined aortic valve pathology. J Thorac Cardiovasc Surg. 2015;149(4):e59-e60. doi:10.1016/j. jtcvs.2015.01.002

37. Rohlffs F, Tsilimparis N, Detter C, et al. New advances in endovascular therapy: Endovascular repair of a chronic DeBakey type II aortic dissection with a scalloped stent-graft designed for the ascending aorta. J Endovasc Ther. 2016;23(1):182-185. doi:10.1177/1526602815621063

38. Muetterties CE, Conklin JH, Moser GW, et al. Right axillary artery cannulation for endovascular repair of an acute type A aortic dissection. Innov Technol Tech Cardiothorac Vasc Surg. 2017;12(2):140-143. doi:10.1097/ IMI.0000000000000350

39. Kong M, Qian J, Duan Q, et al. In situ laser fenestration for revascularization of aortic arch during treatment for iatrogenic type A aortic dissection. J Cardiothorac Surg. 2019;14(1):1-4. doi:10.1186/s13019-019-0877-z 
Current status of type A aortic dissection management

40. Wamala I, Heck R, Falk V, et al. Endovascular treatment of acute type A aortic dissection in a nonagenarian: stabilization of a short covered stent using a bare-metal stent. Interact Cardiovasc Thorac Surg. 2019;29(6):978980. doi:10.1093/icvts/ivz206

41. Ye C, Chang G, Li S, et al. Endovascular stent-graft treatment for Stanford type A aortic dissection. Eur J VasC Endovasc Surg. 2011;42(6):787-794. doi:10.1016/j.ejvs.2011.08.015

42. Ghoreishi M, Shah A, Jeudy J, et al. Endovascular Repair of Ascending Aortic Disease in High-Risk Patients Yields Favorable Outcome. Ann Thorac Surg. 2020. doi:10.1016/j.athoracsur.2019.07.015

43. Bernardes RC, Navarro TP, Reis FR, et al. Early experience with off-the-shelf endografts using a zone 0 proximal landing site to treat the ascending aorta and arch. J Thorac Cardiovasc Surg. 2014;148(1):105-112. doi:10.1016/j. jtcvs.2013.07.049

44. Roselli EE, Idrees J, Greenberg RK, et al. Endovascular stent grafting for ascending aorta repair in high-risk patients. J Thorac Cardiovasc Surg. 2015;149(1):144-154. doi:10.1016/j.jtcvs.2014.07.109

45. Vallabhajosyula P, Gottret JP, Bavaria JE, et al. Endovascular repair of the ascending aorta in patients at high risk for open repair. J Thorac Cardiovasc Surg. 2015;149(2):S144-S150. doi:10.1016/j.jtcvs.2014.07.063

46. Li Z, Lu Q, Feng R, et al. Outcomes of Endovascular Repair of Ascending Aortic Dissection in Patients Unsuitable for Direct Surgical Repair. J Am Coll Cardiol. 2016;68(18):1944-1954. doi:10.1016/j.jacc.2016.08.031

47. Nienaber CA, Sakalihasan N, Clough RE, et al. Thoracic endovascular aortic repair (TEVAR) in proximal (type A) aortic dissection: Ready for a broader application? J Thorac Cardiovasc Surg. 2017;153(2):S3-S11. doi:10.1016/j. jtcvs.2016.07.078

48. Murakami T, Nishimura S, Hosono M, et al. Transapical Endovascular Repair of Thoracic Aortic Pathology. Ann Vasc Surg. 2017;43:56-64. doi:10.1016/j.avsg.2016.10.054

49. Grieshaber P, Nink N, Roth P, et al. Endovascular treatment of the ascending aorta using a combined transapical and transfemoral approach. J Vasc Surg. 2018;67(2):649-655. doi:10.1016/j.jvs.2017.10.046

50. Hsieh YK, Lee $\mathrm{CH}$. Experience of stent-graft repair in acute ascending aortic syndromes. J Card Surg. 2019;34(10):1012-1017. doi:10.1111/jocs.14181

51. Ueda T, Fleischmann D, Dake MD, et al. Incomplete endograft apposition to the aortic arch: Bird-beak configuration increases risk of endoleak formation after thoracic endovascular aortic repair. Radiology. 2010;255(2):645652. doi:10.1148/radiol.10091468

52. Sievers H-H, Rylski B, Czerny M, et al. Aortic dissection reconsidered: type, entry site, malperfusion classification adding clarity and enabling outcome prediction. Interact Cardiovasc Thorac Surg. 2019;30:451-457. doi:10.1093/icvts/ivz281

53. Fanelli F, Cannavale A, O'Sullivan GJ, et al. Endovascular Repair of Acute and Chronic Aortic Type B Dissections Main Factors Affecting Aortic Remodeling and Clinical Outcome. JACC Cardiovasc Interv. 2016;9(2):183-191. doi:10.1016/j.jcin.2015.10.027

54. Rylski B, Milewski RK, Bavaria JE, et al. Outcomes of surgery for chronic type a aortic dissection. Ann Thorac Surg. 2015;99(1):88-93. doi:10.1016/j.athoracsur.2014.07.032

55. Yoshitake A, Okamoto K, Yamazaki M, et al. Comparison of aortic arch repair using the endovascular technique, total arch replacement and staged surgery. Eur J Cardio-thoracic Surg. 2017;51(6):1142-1148. doi:10.1093/ejcts/ ezx028

56. Kanaoka Y, Ohki T, Shukuzawa K, et al. Outcomes of Chimney Thoracic Endovascular Aortic Repair for an Aortic Arch Aneurysm. Ann Vasc Surg. 2019. doi:10.1016/j.avsg.2018.12.087

57. Melissano G, Tshomba Y, Bertoglio L, et al. Analysis of stroke after TEVAR involving the aortic arch. Eur J Vasc Endovasc Surg. 2012;43(3):269-275. doi:10.1016/j.ejvs.2011.12.009

58. Dumfarth J, Kofler M, Stastny L, et al. Stroke after emergent surgery for acute type A aortic dissection: Predictors, outcome and neurological recovery. Eur J Cardio-thoracic Surg. 2018;53(5):1013-1020. doi:10.1093/ejcts/ ezx465

59. Williams DM, Andrews JC, Victoria Marx M, et al. Creation of Reentry Tears in Aortic Dissection by Means of Percutaneous Balloon Fenestration: Gross Anatomic and Histologic Considerations. J Vasc Interv Radiol. 1993;4(1):75-83. doi:10.1016/S1051-0443(93)71824-2 
Endovascular ascending aortic repair in type A aortic dissection: a systematic review.

60. Patel HJ, Williams DM, Meekov M, et al. Long-term results of percutaneous management of malperfusion in acute type B aortic dissection: Implications for thoracic aortic endovascular repair. J Thorac Cardiovasc Surg. 2009;138(2):300-308. doi:10.1016/j.jtcvs.2009.01.037

61. Kamman A V., Yang B, Kim KM, et al. Visceral Malperfusion in Aortic Dissection: The Michigan Experience. Semin Thorac Cardiovasc Surg. 2017;29(2):173-178. doi:10.1053/j.semtcvs.2016.10.002

62. Yang B, Norton EL, Rosati CM, et al. Managing patients with acute type A aortic dissection and mesenteric malperfusion syndrome: A 20-year experience. J Thorac Cardiovasc Surg. 2019;158(3):675-687.e4. doi:10.1016/j. jtcvs.2018.11.127

63. Yang B, Rosati CM, Norton EL, et al. Endovascular Fenestration/Stenting First Followed by Delayed Open Aortic Repair for Acute Type A Aortic Dissection With Malperfusion Syndrome. Circulation. 2018;138(19):2091-2103. doi:10.1161/CIRCULATIONAHA.118.036328

64. Omura A, Matsuda H, Matsuo J, et al. Endovascular repair of thrombosed-type acute Type A aortic dissection with critical renal artery malperfusion. Eur J Cardio-thoracic Surg. 2018;54(6):1142-1144. doi:10.1093/ejcts/ ezy183

65. Zubair MM, de Beaufort HW, Belvroy VM, et al. Impact of Cardiac Cycle on Thoracic Aortic Geometry - Morphometric Analysis of ECG Gated Computed Tomography. Ann Vasc Surg. 2019:1-9. doi:10.1016/j.avsg.2019.10.072

66. Figueroa CA, Taylor CA, Yeh V, et al. Preliminary 3D computational analysis of the relationship between aortic displacement force and direction of endograft movement. J Vasc Surg. 2010;51(6):1488-1497. doi:10.1016/j. jvs.2010.01.058

67. Lam SK, Fung GSK, Cheng SWK, et al. A computational study on the biomechanical factors related to stent-graft models in the thoracic aorta. Med Biol Eng Comput. 2008;46(11):1129-1138. doi:10.1007/s11517-008-0361-8

68. Cook Medical Inc. Cook Zenith Alpha ${ }^{\mathrm{TM}}$ Thoracic Endovascular Graft. Instructions for Use. 2019.

69. Cook Medical Inc. Zenith ${ }^{\circledR}$ TX2 ${ }^{\circledR}$ TAA Endovascular Graft with Pro-Form ${ }^{\text {TM }}$ and the Z-Trak ${ }^{\circledR}$ Plus Introduction System Two-Piece System. 2019.

70. W. L. Gore \& Associates Inc. Instructions for Use GORE ${ }^{\circledast}$ TAG $^{\circledR}$ Conformable Thoracic Stent Graft. 2019.

71. Medtronic Vascular Inc. Thoracic Stent Graft with the Captivia ${ }^{\circledR}$ Delivery System Instructions for Use. 2019.

72. Hughes GC. Stent graft-induced new entry tear (SINE): Intentional and NOT. J Thorac Cardiovasc Surg. 2019;157(1):101-106.e3. doi:10.1016/j.jtcvs.2018.10.060

73. Bischoff MS, Müller-Eschner M, Meisenbacher K, et al. Device Conformability and Morphological Assessment After TEVAR for Aortic Type B Dissection: A Single-Centre Experience with a Conformable Thoracic Stent-Graft Design. Med Sci Monit Basic Res. 2015;21:262-270. doi:10.12659/MSMBR.897010

74. Sugawara J, Hayashi K, Yokoi T, et al. Age-Associated Elongation of the Ascending Aorta in Adults. JACC Cardiovasc Imaging. 2008;1(6):739-748. doi:10.1016/j.jcmg.2008.06.010

75. Hope MD, Hope TA, Crook SES, et al. 4D flow CMR in assessment of valve-related ascending aortic disease. JACC Cardiovasc Imaging. 2011;4(7):781-787. doi:10.1016/j.jcmg.2011.05.004

76. Youssefi P, Gomez A, He T, et al. Patient-specific computational fluid dynamics-assessment of aortic hemodynamics in a spectrum of aortic valve pathologies. J Thorac Cardiovasc Surg. 2017;153(1):8-20.e3. doi:10.1016/j. jtcvs.2016.09.040

77. Kilner PJ, Yang GZ, Mohiaddin RH, et al. Helical and retrograde secondary flow patterns in the aortic arch studied by three-directional magnetic resonance velocity mapping. Circulation. 1993;88:2235-2247. doi:10.1161/01. CIR.88.5.2235

78. Figueroa CA, Taylor CA, Chiou AJ, et al. Magnitude and direction of pulsatile displacement forces acting on thoracic aortic endografts. J Endovasc Ther. 2009;16(3):350-358. doi:10.1583/09-2738.1

79. Cheng SWK, Lam ESK, Fung GSK, et al. A computational fluid dynamic study of stent graft remodeling after endovascular repair of thoracic aortic dissections. J Vasc Surg. 2008;48(2):303-310. doi:10.1016/j.jvs.2008.03.050

80. Tolenaar JL, Jonker FHW, Moll FL, et al. Influence of oversizing on outcome in thoracic endovascular aortic repair. J Endovasc Ther. 2013;20(6):738-749. doi:10.1583/13-4388MR.1

81. Shimono T, Kato N, Yasuda F, et al. Transluminal stent-graft placements for the treatments of acute onset and chronic aortic dissections. Circulation. 2002;106(13 SUPPL.):241-247. doi:10.1161/01.cir.0000032877.55215.59 
Current status of type A aortic dissection management

82. Chen Y, Zhang S, Liu L, et al. Retrograde Type A Aortic Dissection After Thoracic Endovascular Aortic Repair: A Systematic Review and Meta-Analysis. J Am Heart Assoc. 2017;6(9):1-11. doi:10.1161/JAHA.116.004649

83. Liu L, Zhang S, Lu Q, et al. Impact of oversizing on the risk of retrograde dissection after TEVAR for acute and chronic type B dissection. J Endovasc Ther. 2016;23(4):620-625. doi:10.1177/1526602816647939

84. Kreibich M, Rylski B, Kondov S, et al. Endovascular treatment of acute Type A aortic dissection-the Endo Bentall approach. J Vis Surg. 2018;4(19):69-69. doi:10.21037/jovs.2018.03.14

85. Felipe Gaia D, Bernal O, Castilho E, et al. First-in-Human Endo-Bentall Procedure for Simultaneous Treatment of the Ascending Aorta and Aortic Valve. JACC Case Reports. 2020;2(3):480-485. doi:10.1016/j.jaccas.2019.11.071

\section{APPENDIX}

\section{Medline search:}

(endovascular OR Transluminal OR "endovascular procedures"[MeSH Terms] OR endovascular repair OR Endovascular treatment OR Endovascular approach OR endovascular stent-graft OR Trans-luminal stent-graft OR TEVAR OR Thoracic endovascular aortic repair OR thoracic endovascular repair) AND ("type A dissection" OR "acute type A dissection" OR "type A aortic dissection" OR "acute type A aortic dissection" OR ascending aortic dissection) AND ("ascending aorta" OR "ascending" OR "aorta"[MeSH Terms] OR "zone 0") 




\section{Part III}

Current status of descending thoracic aortic assessment 


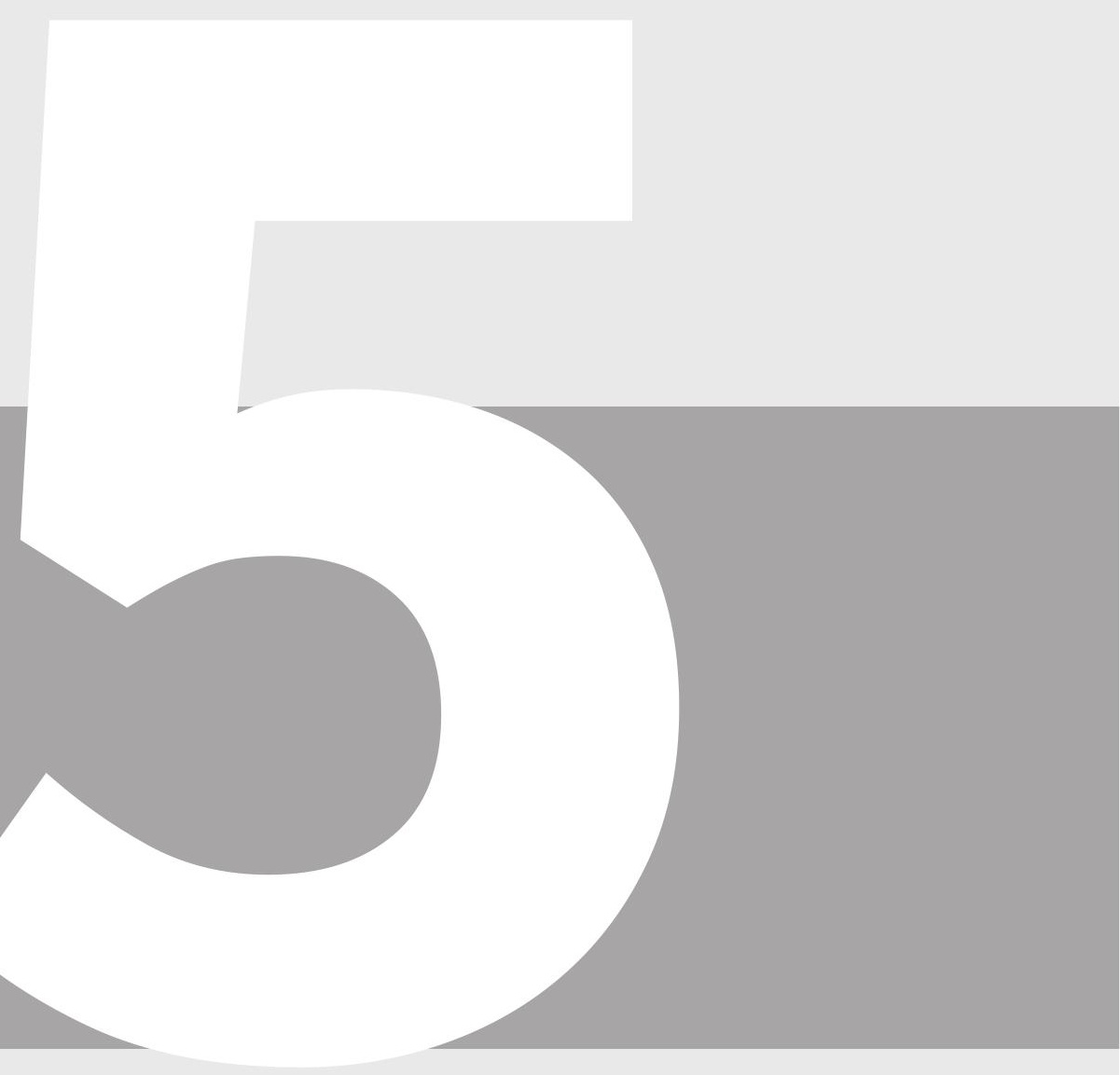




\section{Thoracic endovascular aortic repair:}

a 16-year experience

Ignas B. Houben, MD,

Ilias Errachidi, BSc,

Stijn C.E.V.B. Hazenberg, MD, PhD,

Gert-Jan de Borst, MD, PhD,

Rachael J. Toorop, MD, PhD,

Bart-Jeroen Petri, MD, PhD,

Eline S. van Hattum, MD, PhD,

T. Bloemert-Tuin, MPA,

Frans L. Moll, MD, PhD,

Joost A. van Herwaarden, MD, PhD.

Department of Vascular Surgery, University Medical Center Utrecht, Utrecht, The Netherlands European Journal for Vascular and Endovascular Surgery (submitted) 


\section{ABSTRACT}

\section{Objectives}

The aim of this study is to describe single center long-term outcomes for TEVAR.

\section{Methods}

We conducted a single center retrospective cohort analysis including all adult patients who underwent TEVAR between 2004 and 2019. One hundred fifty-two consecutive patients received TEVAR, of whom 139 patients survived the first 30 postoperative days. These 139 were divided into patients with degenerative aneurysmal disease (group $1, n=75)$, traumatic aortic lesions (group 2, $n=20$ ), or non-aneurysmal, non-traumatic patients (group $3, n=44$ ). Primary endpoints of the analysis were mortality and reintervention rates. Secondary endpoints were neurologic impairment and endoleaks.

\section{Results}

Overall mean follow-up was $4.4 \pm 3.6$ years. 52 patients died after 30 days ( $37.4 \%$ ), with 49\% mortality in group 1 over a mean 4.1 years, $10 \%$ mortality in group 2 over a mean 5.8 years and $30 \%$ mortality in group 3 over a mean 4.1 years $(p=0.002)$. Thoracic aorta related death occurred in two patients (1.4\%) at 40 and 190 days after TEVAR. No permanent disabling spinal cord ischemia occurred and three patients $(2.2 \%)$ had neurologic disability after 30 days, of whom two recovered fully with rehabilitation at 2 months post-TEVAR. Twenty-four patients (17.3\%) required one or more late reinterventions at an average of 2.2 years follow-up, ranging 31 days to 9.1 years after TEVAR. Five year reintervention-free survival rates were $49.7 \%, 81.6 \%$ and $56.4 \%$ for group 1 to 3 respectively $(p=0.012)$.

\section{Conclusions}

Our mid-to-long-term single center retrospective analysis revealed acceptable mortality rates and late reintervention rates were comparable to other long-term TEVAR reports. An extension of the follow-up interval, or follow-up only when symptomatic may be considered after 10 years. Trauma related aortic pathology requiring TEVAR could potentially be followed less extensively after three years, in order to mitigate unnecessary radiation exposure and costs. 


\section{INTRODUCTION}

For thoracic descending aortic pathologies, thoracic endovascular aortic repair (TEVAR) is considered the preferable intervention over open surgery due to the patient's relatively short hospital stay and the acceptable short-term mortality and morbidity. ${ }^{1,2}$ Numerous studies provide promising short-term outcomes for TEVAR. ${ }^{1-10}$

Despite roughly 30 years of worldwide experience, challenges and pitfalls of endovascular aortic therapy remain. Serious complications that require a better understanding include renal-, spinal-, cerebral- and visceral ischemia. Moreover, endoleaks are still a big challenge and regularly require reintervention. ${ }^{1,2,11}$

Although the short-term results of TEVAR are encouraging, long-term results with a large number of patients have not been well assessed. Knowledge of long-term outcomes weighs heavily in surgical decision making. Endovascular therapy in the abdominal aorta is well-advanced compared to the thoracic aortic region, especially in quantity and availability of long-term data. It would seem reasonable to watch and learn from the results obtained there. However, thus far we have learned that the abdominal and thoracic aortic regions are two different entities, with specific biomechanics and a variety of particular challenges. For instance, type II endoleaks are more prevalent in the abdominal aortic region ${ }^{12}$ and the thoracic aortic wall is more compliant than the abdominal aortic wall. ${ }^{13} \mathrm{~A}$ thoracic endovascular long-term analysis is therefore paramount. This study describes a single center experience, assessing the outcomes of 30 day postoperative survivors in patients after TEVAR over a period of 16 years.

\section{METHODS}

Approval for this study was obtained from the institutional review board (Utrecht University Medical Center protocol number 21-079/C, date of approval February 03, 2021), the need for patient consent was waived. All consecutive patients undergoing TEVAR between January 2004 and December 2019 were retrospectively analyzed. Exclusion criteria consisted of age under 18 years, and secondary TEVAR procedures after prior thoracic endovascular repair in another center. Furthermore, all patients having TEVAR with the main device reaching further distal than the celiac trunk (grafts with abdominal branches and/or fenestrations) were excluded.

\section{Clinical data}

Clinical data was gathered from the electronic medical records. Baseline data included body length and weight, body-mass index (BMI), history of hypertension, dyslipidemia, diabetes mellitus, chronic obstructive pulmonary disease, coronary artery disease, re- 
nal failure, history of tobacco use and history of cerebral or myocardial ischemic event. Information on prior cardiovascular interventions, including aortic repair was obtained. Baseline physical status of the patients was evaluated with the American Society of Anesthesiologists (ASA) score from the anesthesiology pre-operative screening reports. Urgent TEVAR was defined as TEVAR within 48 hours after presentation. Emergent TEVAR was defined as TEVAR within 24 hours after presentation. Patients were subdivided into three groups, being patients with degenerative aneurysmal disease (group 1), patients with traumatic aortic lesions (group 2), and non-aneurysmal, non-traumatic patients (group 3).

\section{Follow-up}

All 30 day survivors were analyzed. Mid- to long-term all-cause mortality, thoracic aortic related mortality and freedom from thoracic aortic related reinterventions were our main outcomes. When patients had been lost to follow-up, additional follow-up data on mortality and freedom from thoracic aortic reintervention were gathered from contacting referral hospitals and first line healthcare practitioners. Late endoleak was registered and was defined as endoleak after 30 days.

\section{Statistical analysis}

Continuous data was presented as mean \pm SD and categorical data was presented as frequency and percentage. If a variable had more than ten percent missing data, this was specified in the tables. Normality of the distribution of the data was tested using the Shapiro-Wilk test. Differences in clinical data between groups was assessed using chi-square analysis for categorical data and t-test or one-way ANOVA for continuous data. To examine the variable differences between the three main indication groups for non-normally distributed, continuous data, a Mann-Whitney $U$ test was applied. Statistical significance was defined as $p<0.05$. Analysis of the data was performed using SPSS Statistics 27 (IBM, Armonk, NY) and R3.6.2 (R Core Team, 2019).

\section{RESULTS}

One hundred fifty-two consecutive patients received TEVAR, of whom 139 patients survived the first 30 postoperative days. These 139 patients (92 male, mean age $63.8 \pm$ 21.2 years) were divided into patients with degenerative aneurysmal disease (group $1, \mathrm{n}$ $=75$, mean age $69.4 \pm 9.0$ years), traumatic aortic lesions (group 2, $n=20$, mean age $35.2 \pm$ 13.3 years), or non-aneurysmal, non-traumatic patients (group 3, $n=44$, mean age $67.4 \pm$ 12.0 years). Figure 1 shows the distrubtion of procedures over the years. Figure 2 shows the distribution of indications for TEVAR. Patient demographics and comorbidities are outlined in Table 1, with the main groups depicted as separate columns. In group 1, 
four patients (5.3\%) were treated urgently. In group 2, all patients were treated urgently and in group 3, 17 patients (38.6\%) were treated urgently. Overall, mean follow-up time for mortality and reinterventions was $4.4 \pm 3.6$ years. Main outcomes after 30 days are summarized in Table 2.

Figure 1. Number of TEVAR procedures per year

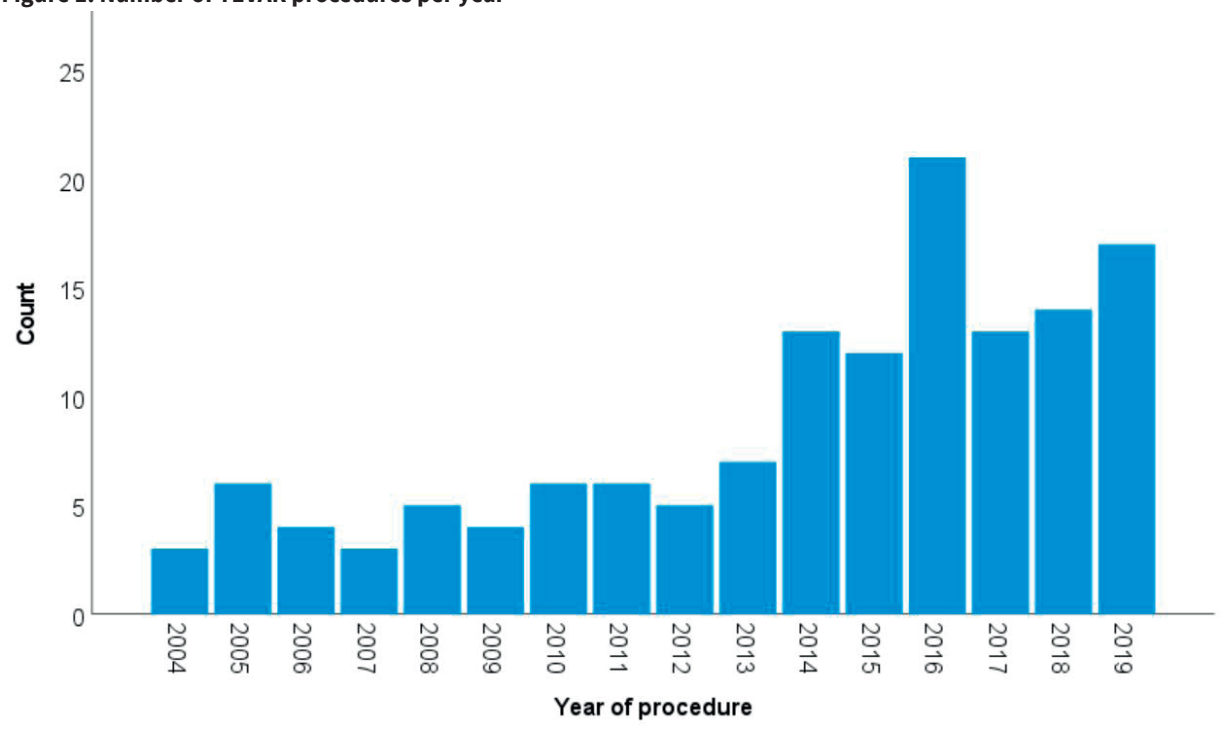

Figure 2. Main indications for TEVAR

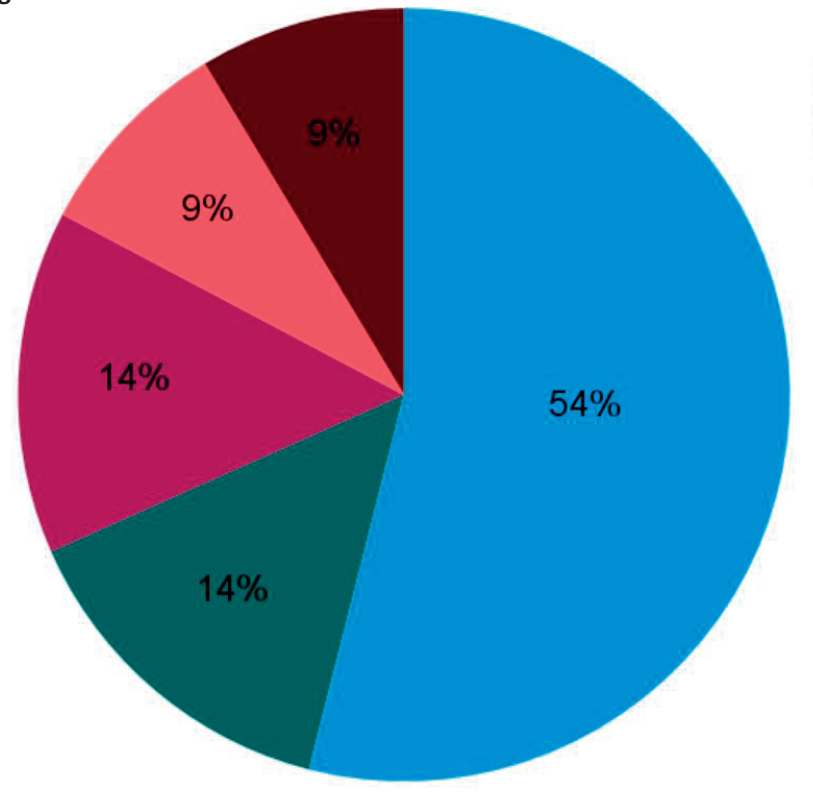

TEVAR indication

Thoracic aortic aneurysm

- Acute type B aortic dissection

- Traumatic aortic rupture / dissection

Chronic aortic dissection

Ether 
Current status of descending thoracic aortic assessment

Table 1. Demographics and comorbidities

\begin{tabular}{|c|c|c|c|c|c|}
\hline Demographics and comorbidities & $\begin{array}{c}\text { All } \\
(n=139)\end{array}$ & $\begin{array}{c}\text { Aneurysm } \\
(n=75)\end{array}$ & $\begin{array}{l}\text { Trauma } \\
(n=20)\end{array}$ & $\begin{array}{l}\text { Other } \\
(n=44)\end{array}$ & $\mathbf{p}$ \\
\hline Age (year), mean \pm SD & $63.8 \pm 21.2$ & $69.4 \pm 9.0$ & $35.2 \pm 13.3$ & $67.4 \pm 12.0$ & $<0.001$ \\
\hline Male sex, n (\%) & $92(66)$ & $48(64)$ & $17(85)$ & $27(61)$ & 0.151 \\
\hline Length (meter), mean \pm SD & $1.74 \pm 0.09$ & $1.72 \pm 0.08$ & $1.81 \pm 0.09$ & $1.75 \pm 0.10$ & 0.002 \\
\hline Weight (kilogram), mean $\pm \mathrm{SD}^{\star}$ & $76.8 \pm 14.8$ & $77.7 \pm 12.8$ & $88.3 \pm 7.6$ & $74.2 \pm 18.2$ & 0.211 \\
\hline BMI, (kilogram/m2), mean $\pm \mathrm{SD}^{\star}$ & $25.6 \pm 4.4$ & $26.1 \pm 3.6$ & $26.5 \pm 0.7$ & $24.5 \pm 5.8$ & 0.203 \\
\hline Hypertension, n (\%) & $85(61)$ & $58(77)$ & 0 & $27(61)$ & $<0.001$ \\
\hline Dyslipidemia, n (\%) & $29(21)$ & $20(27)$ & 0 & $9(20)$ & 0.033 \\
\hline Diabetes mellitus, n (\%) & $21(15)$ & $16(21)$ & $1(5)$ & $4(9)$ & 0.078 \\
\hline Chronic obstructive pulmonary disease, $\mathrm{n}(\%)$ & $28(20)$ & $21(28)$ & $1(5)$ & $6(14)$ & 0.032 \\
\hline Peripheral artery disease, $\mathrm{n}(\%)$ & $28(20)$ & $24(32)$ & 0 & $4(9)$ & 0.001 \\
\hline Coronary artery disease, $\mathrm{n}(\%)$ & $33(24)$ & $26(35)$ & 0 & $7(16)$ & 0.002 \\
\hline Smoking, prior or current, $\mathrm{n}(\%)^{*}$ & $84(68)$ & $54(78)$ & $5(33)$ & $25(63)$ & 0.002 \\
\hline Prior aortic valve repair, $\mathrm{n}(\%)$ & $7(5)$ & $4(5)$ & $1(5)$ & $2(5)$ & 0.982 \\
\hline Prior thoracic aortic repair, $\mathrm{n}(\%)$ & $17(12)$ & $10(13)$ & 0 & $7(16)$ & 0.180 \\
\hline Prior abdominal aortic repair, $\mathrm{n}(\%)$ & $23(17)$ & $18(24)$ & 0 & $5(11)$ & 0.020 \\
\hline Prior coronary artery bypass graft, n (\%) & $8(6)$ & $7(9)$ & 0 & $1(2)$ & 0.137 \\
\hline Prior myocardial infarction, n (\%) & $18(13)$ & $15(20)$ & 0 & $3(7)$ & 0.021 \\
\hline Prior renal failure, n (\%) & $27(19)$ & $19(25)$ & 0 & $8(18)$ & 0.038 \\
\hline Prior cerebral ischemia, n (\%) & $21(15)$ & $18(24)$ & 0 & $3(7)$ & 0.005 \\
\hline ASA-classification $\geq 3, \mathrm{n}(\%)^{\star}$ & $62(65)$ & $44(72)$ & $4(57)$ & $14(50)$ & 0.117 \\
\hline
\end{tabular}

* $>10 \%$ cases missing

Table 2. Postoperative outcomes before and after 30 days

\begin{tabular}{|c|c|c|c|c|c|}
\hline Outcomes $>30$ days post-TEVAR ${ }^{\star}$ & $\begin{array}{c}\text { All } \\
(n=139)\end{array}$ & $\begin{array}{c}\text { Aneurysm } \\
(n=75)\end{array}$ & $\begin{array}{l}\text { Trauma } \\
(n=20)\end{array}$ & $\begin{array}{l}\text { Other } \\
(n=44)\end{array}$ & $\mathbf{p}$ \\
\hline Overall follow-up, mean years \pm SD & $4.4 \pm 3.6$ & $4.1 \pm 3.0$ & $5.8 \pm 4.9$ & $4.1 \pm 3.6$ & 0.177 \\
\hline Mortality, n (\%) & $52(37)$ & $37(49)$ & $2(10)$ & $13(30)$ & 0.002 \\
\hline TEVAR related reintervention, $\mathrm{n}$ & 14 & 9 & 2 & 3 & 0.551 \\
\hline Disease progression related reintervention, $\mathrm{n}$ & 14 & 4 & 1 & 9 & 0.155 \\
\hline Endoleak, n (\%) & $11(8)$ & $8(11)$ & 0 & $3(7)$ & 0.522 \\
\hline
\end{tabular}

* Endoleaks or reinterventions occurring at $\leq 30$ days post-TEVAR excluded

\section{Survival}

During the average 4.4 year follow-up, 52 patients (37\%) of all 30-day survivors died. One- and five-year survival rates were $92.0 \%$ and $63.6 \%$. Aorta-related deaths occurred in three patients (2.2\%). Two of these were thoracic aorta related and occurred at 40 days and 190 days post-TEVAR. The patient dying 40 days after transapical TEVAR, was readmitted with a hemothorax, requiring an evacuation through thoracotomy after 
20 days, complicated by pneumonia, multiple organ failure and subsequently death. The patient dying after 190 days suffered from mediastinitis secondary to a periaortic abscess following an antegrade TEVAR through a small thoracotomy. The patient died from secondary heart failure after sepsis. One patient died from an abdominal aortic aneurysm rupture, more than three years after TEVAR. A

Kaplan-Meier survival curve is shown in Figure 3, grouped by main indication groups. It reveals only two deaths total in group 2 . One patient died from a cranial skull graft infection 96 days after TEVAR. The other patient suffering from lung cancer, deceased almost three years after TEVAR. Between group 1 and 3, overall survival revealed no significant difference using a log-rank test $(\mathrm{p}=0.139)$. Five-year survival rates for group 1,2 and 3 respectively were $55.8 \%, 87.7 \%$ and $69.0 \%$.

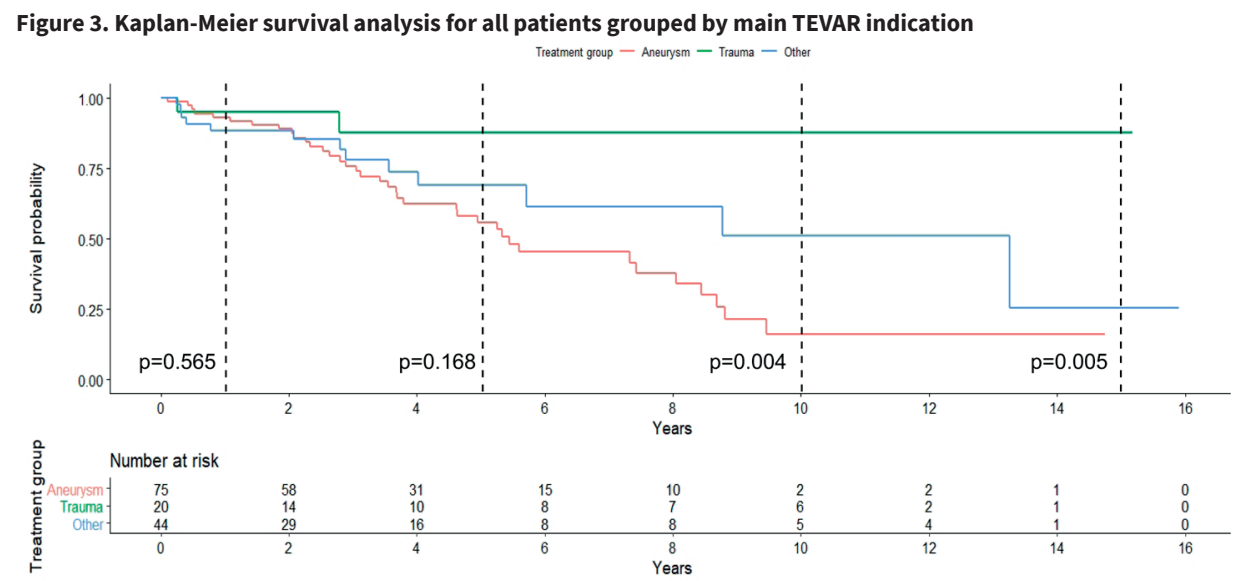

\section{Reintervention}

Beyond 30 days, 125 patients (89.9\%) did not have any reintervention and were still alive. Twenty-four patients out of 139 30-day survivors (17.3\%) required one or more late reinterventions at an average of 2.2 years follow-up, ranging 31 days to 9.1 years after TEVAR. Thirteen of these 24 patients required a total of 14 reinterventions due to disease progression. Eleven of these 24 patients required a total of 14 procedure-related reinterventions. Indications for these procedure-related reinterventions are summarized in Table 3. Out of all 14 TEVAR-related late reinterventions, only two reinterventions occurred after two years. The first was a saccular mycotic aortic arch aneurysm following two years after TEVAR due to stent graft infection with staphylococcus aureus. At the same time, a para-aortic abscess was drained transesophageally and an esophageal stent was placed. Due to progressive arch aneurysm growth, a proximal TEVAR extension was performed to cover the saccular aneurysm after abscess drainage. Additionally, 
an esophageal resection had to be performed due to persistent aorto-esophageal fistula following the transesophageally drained para-aortic abscess. Four years following these reinterventions, the patient is still doing well with an esophageal reconstruction conduit and a stable aorta. The second patient needed reintervention for a recurrent type IIIa endoleak between the frozen elephant trunk and the proximal stent graft 2.9 years after the index procedure. Overall late reintervention rates for the patients in the three main groups of 30-day survivors were 11 out of 75 for group 1 (14.6\%), three out of 20 for group 2 (15\%), and 10 out of 44 for group $3(22.7 \%, p=0.510)$.

Table 3. Indications for TEVAR related reinterventions after 30 days.

\begin{tabular}{lccc}
\hline Indications for reinterventions & $\begin{array}{c}\text { Aneurysm } \\
(\mathbf{n = 7 5 )}\end{array}$ & $\begin{array}{c}\text { Trauma } \\
(\mathbf{n = 2 0 )}\end{array}$ & $\begin{array}{c}\text { Non-traumatic non- } \\
\text { aneurysm }(\mathbf{n = 4 4 )}\end{array}$ \\
\hline Type I endoleak & 2 & 0 & 1 \\
Type III endoleak & 1 & 0 & 0 \\
\hline Aortic occlusion & 0 & 2 & 1 \\
Arterial occlusion & 3 & 0 & 0 \\
Mycotic aneurysm & 1 & 0 & 0 \\
Aortic rupture & 0 & 0 & 1 \\
Access site complications* & 2 & 0 & 0 \\
*(infection/hemorrhage/pseudoaneurysm) & & & \\
\hline
\end{tabular}

Two out of three reinterventions in group 2 were performed relatively early after 67 and 91 days postoperatively, due to partial collapse of the aortic stent graft in one patient and a type $1 \mathrm{a}$ EL in the other patient respectively. Both patients had no clinical symptoms. The first patient was treated by open arch repair and explantation of the stent graft and is doing well 15 years after the reintervention. The second patient received a custom made extension with a stent graft with fenestrations for the BCT and the LCCA origins. The patient is also doing well one year after the reintervention. The third patient presented because of acute paralysis in both legs 21 months after TEVAR. This patient suffered an acute in-stent complete occlusion. He was emergently treated with open explantation and descending thoracic aorta tube repair through left thoracotomy. Five years later the patient has been doing well and is biannually followed.

Five year reintervention-free survival rates per group were $49.7 \%, 81.6 \%$ and $56.4 \%$ respectively ( $p=0.012$, Figure 4). A multivariate regression exploring the effects of age, sex and arch involvement on reintervention-free survival in the first treatment group versus the third treatment group, age was shown to be significant predictor for reintervention or death with a p-value of 0.019 (Table 4). However, when performing a similar regression analysis with reintervention as the main outcome parameter, age was found to lose 

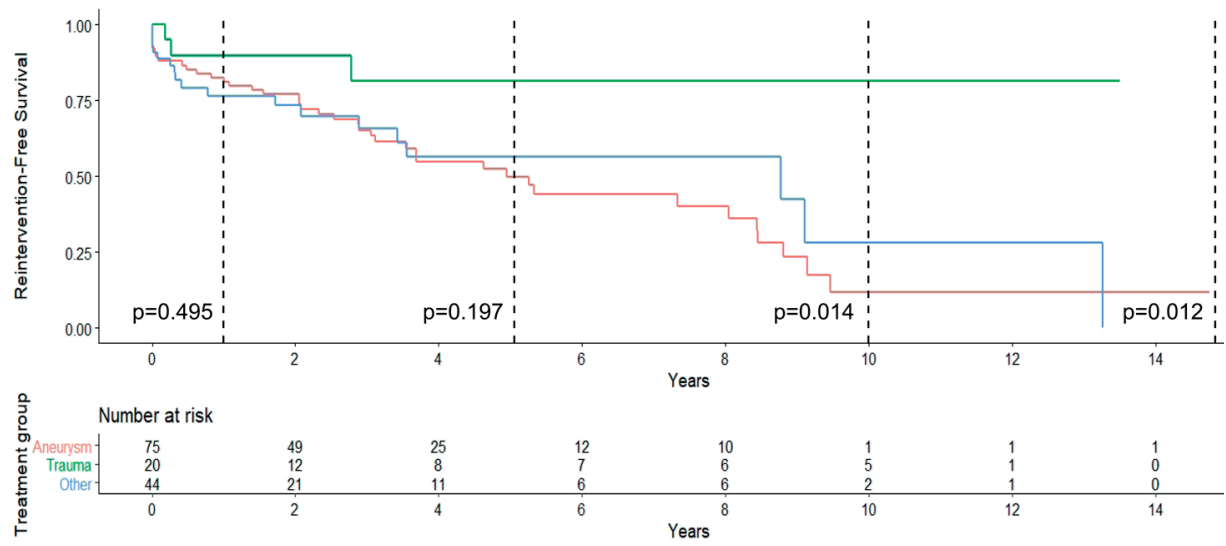

Table 4. Multivariate logistic regression with reintervention or death as primary outcome measure, where all trauma cases were excluded.

\begin{tabular}{llll}
\hline Variable & Odds Ratio & $\mathbf{9 5 \%} \mathbf{C l}$ & p-value \\
\hline Age, years & 1.049 & $1.008 ; 1.093$ & 0.019 \\
Female gender & 0.828 & $0.376 ; 1.822$ & 0.639 \\
Aneurysm & 1.420 & $0.646 ; 3.121$ & 0.383 \\
Arch involvement & 2.227 & $0.941 ; 5.269$ & 0.069 \\
\hline
\end{tabular}

significance as a predictor $(p=0.229)$. The annual reintervention rate after the first year was $0-6 \%$, with the ninth year as an exception ( 2 reinterventions in 14 remaining patients; reintervention proportion 17\%) and no more reinterventions occurring after nine years. Out of a total of 28 reinterventions performed after 30 days, eleven (39\%) were performed in the first year post-TEVAR. All patients survived 30 days following reintervention and none of the patients after reintervention died from a thoracic aortic related cause during follow-up. For each group, annual reintervention rates remained below $6 \%$ between year one and seven post-TEVAR. Five years after TEVAR, follow-up data was present in 43 patients. In these patients 43 patients, three patients received re-TEVAR after five years (7.0\%). All three reinterventions were elective reinterventions, performed because of disease progression. One 66 year old female suffered an initial type B aortic dissection for which she received successful TEVAR in the descending thoracic aorta. Nine years later, she suffered a type A aortic dissection, for which she received open supra coronary ascending aorta replacement, leaving a pseudoaneurysm at the subclavian artery, which was successfully covered three months later using a custom-made stent graft. Currently the patient is alive with a stable aorta. One 59 year old female had received initial TEVAR for a thoracic aortic aneurysm through an infrarenal aortic conduit. Nine years later, the patient had developed a thoracoabdominal aneurysm 
distal to the previous TEVAR, for which fenestrated TEVAR was attempted. Introduction of the fenestrated stent graft, however, caused rupture of the small external iliac artery. This was endovascularly managed with covering of the hypogastric artery with a stent graft. Considering the increased risk on spinal cord ischemia ( $\mathrm{SCl}$ ), especially when the fenestrated TEVAR would be performed in hemodynamically poor conditions, the procedure was discontinued. Now, 5.5 years later the patient is alive and doing well. The third long-term reintervention was performed in a male patient who had received initial TEVAR for a descending thoracic aortic aneurysm. Eight years after his initial TEVAR, he revealed a type la endoleak with aneurysm growth, because of diameter increase of the aneurysm neck. The patient received an endovascular proximal extension, combined with a chimney into the LSA. The procedure was uncomplicated and the patient could be discharged in a good clinical state, but was readmitted a month later with a sepsis, without a clear source of infection and a potential stent graft infection for which he was then treated with antibiotics. Another month later the patient developed bilateral pulmonary emboli and bilateral pneumonia. A few days later the patient died at age 81 .

During follow-up, there was one alleged subclavian steal case. One patient, who underwent branched TEVAR with branches to the brachiocephalic trunk and to the LCCA, had her LSA covered and had a late presentation of symptoms resembling subclavian steal syndrome. After 1.5 years she returned to the vascular surgeon with complaints of dizziness and functional loss of her left arm. An axillo-axillary bypass was performed, which relieved complaints in her left arm, but did not relieve her dizziness. In the two years after her axillo-axillary bypass procedure, CTA follow-up revealed an occlusion of the branched stent in the LCCA, although no signs of cerebral ischemia were seen. She was eventually diagnosed with starting unrelated dementia two years after her bypass procedure.

\section{Neurologic disability}

The annual incidence of stroke was 3.3\% during the entire study period, including the perioperative strokes. Three patients suffered disabling neurological impairment that persisted beyond 30 days. One of these patients underwent TEVAR covering the LSA with concomitant chimney placement in both the left common carotid artery (LCCA) and the brachiocephalic trunk (BCT). Another patient received branched TEVAR with revascularization of both the BCT and the LCCA. In both patients the level of disability had almost completely disappeared within two months. The third patient suffered from a lusorian artery aneurysm and underwent TEVAR with concomitant chimney placement in both subclavian arteries. He suffered from a postoperative superior vena cava syndrome. On the sixth postoperative day he was weaned of sedation and a right sided hemiparesis was noted and cerebral CT scan revealed multiple cerebellar infarctions, a right occipital 
infarction and a left frontal infarction, which had not been seen on a cerebral MRI one month earlier. The patient regained function, but required a walker for the rest of his life until death 3.7 years later. There was a significant difference between having intraoperative additional arch vessel procedures and having no intraoperative additional arch vessel procedures in the occurrence of short- and long-term post cerebral ischemia ( 3 out of 17 versus 3 out of $122, \mathrm{p}=0.04)$. Temporary disability due to $\mathrm{SCl}$ occurred in three patients and disappeared within 30 days. A fourth patient suffering from $\mathrm{SCl}$ died on the fourth postoperative day due to irreversible visceral ischemia. Therefore, no permanent disability after 30 days was seen.

\section{Endoleaks}

In the series of 75 aneurysm patients, there were 30 early endoleaks, of which 22 spontaneously disappeared within one year and an additional two type II endoleak required no reintervention as the endoleak did not lead to aneurysm growth during follow-up. One patient with type la endoleak died within a year following TEVAR, due to cardiopulmonary consequences of pneumonia and heart failure, which appeared not TEVAR related. Out of 30 patients with early endoleak, eventually four patients required a reintervention. Two type $\mathrm{Ib}$ endoleaks received a distal extension both at one year follow-up. One type II endoleak lead to slow aneurysm growth, prompting reintervention after two years. One patient showed a type IIla endoleak between the proximal stent graft and the earlier placed frozen elephant trunk, which disappeared spontaneously within six months. However, 2.9 years after the index procedure the type Illa endoleak reappeared, prompting re-TEVAR. Thus, for early endoleaks in aneurysm patients reinterventions were performed in $0 \%$ of type la, in $100 \%$ of type Ib, $6 \%$ of type II and $14 \%$ of type III endoleaks. Moreover, $86.7 \%$ of endoleaks (26/30) either resolved spontaneously without intervention or did not have interventional consequences. Later emerging endoleaks were seen in six patients during follow-up. One patient showed a type II EL after six months, without significant aneurysm growth, for which the patient has been followed using conservative treatment. One patient showed aneurysm growth and a type II EL after two years, for which endovascular options were discussed. Because of poor condition and low expected rupture rate it was treated conservatively. The patient was found deceased at home 1.5 months later. No obduction was carried out. Another 80 year old male patient, who had a mycotic descending thoracic aneurysm due to a coxiella burnetii infection, had successful TEVAR with decreasing aneurysm diameters after three months. After 18 months however, progression of infection was seen with a type Ib endoleak. A reintervention was deemed a too high risk in a patient with poor condition. The patient was referred back to his initial referral hospital for further cardiovascular management. Two later emerging endoleaks required reintervention. However, one of these two patients, suffering a type la EL after 3 years requested no reintervention. 
The patient died six years later of unknown cause. The last patient revealed a type Ic endoleak at the chimney in a lusorian artery aneurysm. The patient received re-stenting of the lusorian artery.

In the 20 traumatic patients, there were no early or late endoleaks.

In the 44 patients in group 3, six out of eight early endoleaks spontaneously disappeared within approximately one year. The remaining two early endoleaks received immediate re-TEVAR. One patient with type la endoleak required re-TEVAR at postoperative day one, after persistent proximal bleeding from an aorto-esophageal fistula. One year later, he received another stent graft, because of kinking and stenosis within the overlapping two earlier deployed stent grafts. However, due to persistent stenosis, two days after deployment of the third stent graft, the patient received open aortic arch replacement with explantation of the endovascular stent grafts. The second patient suffered a rupture following a type IIla endoleak, requiring re-TEVAR and left-sided thoracotomy for evacuation of hematoma. Sixteen months later, this patient showed a recurrent type IIla endoleak, which ruptured again leaving a hemothorax, for which a another stent graft was placed and thoracic drainage was performed. Both patients are currently alive and doing well. Later emerging endoleaks only occurred in one additional patient. This consisted of a type II endoleak after six months, causing aneurysm growth. The patient wished no reintervention and died two years following initial TEVAR without a clear cause of death. All other reinterventions or complications in this third group were ascribed to disease progression and are summarized in the reintervention paragraph.

\section{DISCUSSION}

As the follow-up of TEVAR treated patients is increasing and the early results have been promising in the past decades, we describe the follow-up of our patients treated over the past 16 years who survived the first 30 days, aiming to reflect on the results over a longer time.

First, we found that indications for TEVAR have a very different effect on mid- and longterm outcomes. Therefore, they had to be further analyzed as separate groups. Second, it appeared evident that thoracic aortic related mortality was not seen beyond 190 days of follow-up after TEVAR. The only aorta-related death identified after this period was an abdominal aortic rupture, three years after the TEVAR procedure. Overall mortality was slightly higher in our cohort, compared to long-term EVAR reports, reporting a 5-year overall survival of $60-70 \% .{ }^{14,15}$ However, our 5-year survival rates for patients in group 
1 and 3 were $55.8 \%$ and $69.0 \%$ respectively, which has been shown to match reports of long-term TEVAR analyses. ${ }^{16-18}$

The question as to whether follow-up is indicated after 30 days depends on the reintervention risk rather than the mortality risk. In our cohort, $17.3 \%$ of surviving patients beyond 30 days underwent a thoracic aortic reintervention. The annual reintervention rate of $0-6 \%$ was in a similar range to that of EVAR, being reported around $3-5 \% .{ }^{19}$ Moreover, our mortality and reintervention rates were comparable to prior long-term TEVAR cohort analyses. ${ }^{1,16,17}$ European guidelines dictate a follow-up regime of six months, twelve months and annually, with the possibility of an interval extension to two-three years in case of a stable condition in the third year of follow-up. ${ }^{20-22}$ Moreover, in the case of dissection, intramural hematoma, penetrating atherosclerotic ulcer or an inflammatory component it is recommended to additionally perform a CTA or MRI follow-up at three months after the procedure. ${ }^{22}$ Since the interval between TEVAR and reintervention requirement was widespread in our cohort, we agree that the recommendations as currently dictated should be pursued, at least until a ten year follow-up period. If the aorta is stable after ten years, further extension of the follow-up interval can be applied, or non-elective follow-up only can be discussed with the patient. A separate approach should be applied in patients receiving a thoracic stent graft following a trauma. Although the number of patients receiving TEVAR after traumatic dissection was small, the number of complications and the TEVAR related mortality rate was convincingly lower than in the other disease groups. The three reinterventions occurring at 67, 91 and 641 days after the index procedure were invasive, requiring open aortic replacement in two out of three cases and having arch vessel involvement in all three cases. All three cases had no more complications during follow-up after reintervention and were still alive at the end of the study period. Currently, guidelines recommend consideration of different imaging modalities, such as X-ray and MRI, due to unnecessary radiation exposure from CTA. ${ }^{20,21}$ However, no guideline reports on follow-up imaging intervals compared to other aortic disease. As the patients undergoing trauma are generally younger with a healthier aorta, having good landing zones we may reconsider the need for imaging surveillance in a stable situation after three years of follow-up altogether.

\section{Neurologic deficits}

Perhaps the most notorious complications after TEVAR are neurologic deficits, being an important reason why we try to minimize endovascular maneuvers in the aortic arch, why we prevent covering unnecessary aortic length with stent grafts, why we aim to avoid blocking of the LSA and otherwise liberally revascularize and why we use a spinal tap in patients with increased risk of $\mathrm{SCl}$. In our cohort, permanent disability as a result of $\mathrm{SCl}$ was very low and disabling symptoms did not surpass 30 days in any patient. 
In the three patients with postoperative cerebral ischemia and disability lasting longer than 30 days, rehabilitation helped two out of three patients regain normal daily life function of their preoperative abilities. Interestingly, all three patients with disability lasting longer than 30 days and requiring active rehabilitation received chimneys or branches to at least one of the branching arch vessels. It was noticeable that our patients treated with additional arch vessel procedures, albeit a low number with 17 out of 139 patients, revealed a significantly higher amount of postoperative cerebral ischemia $(p=0.04)$. The high incidence of stroke in patients receiving TEVAR with endovascular revascularization of supra-aortic target vessels versus patients receiving TEVAR without involvement of the arch vessels has been previously reported. ${ }^{23}$ Compared to previously reported cohort studies on long-term TEVAR, our overall neurologic deficit was low and especially no lasting paraparesis was seen, whereas others report permanent deficits of up to $2 \%$. $^{1,16,17}$

Left upper extremity functional loss was only described in one of our 45 patients undergoing LSA coverage. Prior reporting in literature of left upper extremity ischemia after LSA coverage has been widely varying between $2-12 \%{ }^{24,25}$

\section{Disease progression related reinterventions}

Disease progression related reinterventions, including distal sealing problems in particular, increased as follow-up progressed. As previously described by our group, ${ }^{26}$ we believe that the challenges concerning management of type Ib endoleaks and aneurysm progression distal to the stent graft are an underrated issue in current literature. It is believed that stent grafts in the descending aorta are challenged by an upward force to the distal end of the stent graft causing potential distal sealing problems. This effect may be larger in a more tortuous thoracic aorta with a more pronounced S-shape and should also be kept in mind in older patients as tortuosity increases with age. ${ }^{27} \mathrm{~A}$ potential solution to prevent proximal migration could be the addition of distal endoanchors, in order to mitigate the reintervention risk. ${ }^{28}$

Another theory for the majority of reinterventions being disease progression related, may be the fact that the relatively stiff stent graft causes a higher pressure on the native aorta, which may be first noticed at the sites adjacent to the TEVAR. The discrepancy between stiffness may be most pronounced immediately proximal and distal to the stent graft ends, causing a higher stress and in turn progressive aortic growth. 


\section{CONCLUSION}

This retrospective long-term single center analysis of TEVAR during a 16-year period reveals low thoracic aortic related mortality rates and an annual thoracic aortic reintervention rate of $0-6 \%$ during long-term follow-up. A majority of reinterventions occurring after 30 days accounted to disease progression proximal or distal to the stent graft. Reintervention rates seemed comparable to that in other long-term (T)EVAR reports. No reintervention occurred after 9.1 years and an extension of the follow-up interval, or follow-up only when symptomatic, may be considered after 10 years. Trauma related aortic pathology requiring TEVAR tends to have a good aorta-related survival rate and could potentially be followed less extensively after three years, in order to mitigate unnecessary radiation exposure and costs. 


\section{REFERENCES}

1. Ziza V, Canaud L, Molinari N, Branchereau P, Marty-Ané C, Alric P. Thoracic endovascular aortic repair: A single center's 15-year experience. J Thorac Cardiovasc Surg. 2016;151(6):1595-1603.e7. doi:10.1016/j. jtcvs.2015.12.030

2. Cheng YT, Cheng CT, Wang SY, et al. Long-term Outcomes of Endovascular and Open Repair for Traumatic Thoracic Aortic Injury. JAMA Netw open. 2019;2(2):e187861. doi:10.1001/jamanetworkopen.2018.7861

3. Dake MD, Semba CP, Liddell RP, Miller DC, Mitchell RS, Walker PJ. Transluminal placement of endovascular stent-grafts for the treatment of descending thoracic aortic aneurysms. N Engl J Med. 1994;331(26):1729-1734. doi:10.1056/NEJM199412293312601

4. Leurs LJ, Bell R, Degrieck Y, Thomas S, Hobo R, Lundbom J. Endovascular treatment of thoracic aortic diseases: Combined experience from the EUROSTAR and United Kingdom Thoracic Endograft registries. J Vasc Surg. 2004;40(4):670-679. doi:10.1016/j.jvs.2004.07.008

5. Desai ND, Pochettino A, Szeto WY, et al. Thoracic endovascular aortic repair: Evolution of therapy, patterns of use, and results in a 10-year experience. J Thorac Cardiovasc Surg. 2011;142(3):587-594. doi:10.1016/j. jtcvs.2011.02.050

6. Lee WA, Daniels MJ, Beaver TM, Klodell CT, Raghinaru DE, Hess PJ. Late outcomes of a single-center experience of 400 consecutive thoracic endovascular aortic repairs. Circulation. 2011;123(25):2938-2945. doi:10.1161/ CIRCULATIONAHA.110.965756

7. Riambau V, Zipfel B, Coppi G, et al. Final operative and midterm results of the European experience in the RELAY Endovascular Registry for Thoracic Disease (RESTORE) study. J Vasc Surg. 2011;53(3):565-573. doi:10.1016/j. jvs.2010.09.050

8. Foley PJ, Criado FJ, Farber MA, et al. Results with the Talent thoracic stent graft in the VALOR trial. J Vasc Surg. 2012;56(5):1214-1221.e1. doi:10.1016/j.jvs.2012.04.071

9. Wang GJ, Cambria RP, Lombardi J V., et al. Thirty-day outcomes from the Society for Vascular Surgery Vascular Quality Initiative thoracic endovascular aortic repair for type B dissection project. J Vasc Surg. 2019;69(3):680691. doi:10.1016/j.jvs.2018.06.203

10. Li DL, He YJ, Wang XH, et al. Long-term Results of Thoracic Endovascular Aortic Repair for Type B Aortic Dissection and Risk Factors for Survival. J Endovasc Ther. 2020;27(3):358-367. doi:10.1177/1526602820910135

11. Fairman AS, Beck AW, Malas MB, et al. Reinterventions in the modern era of thoracic endovascular aortic repair. In: Journal of Vascular Surgery. Vol 71. Mosby Inc.; 2020:408-422. doi:10.1016/j.jvs.2019.04.484

12. Nienaber CA, Kische S, Rousseau H, et al. Endovascular repair of type B aortic dissection: Long-term results of the randomized investigation of stent grafts in aortic dissection trial. Circ Cardiovasc Interv. 2013;6(4):407-416. doi:10.1161/CIRCINTERVENTIONS.113.000463

13. Mohiaddin RH, Underwood SR, Bogren HG, et al. Regional aortic compliance studied by magnetic resonance imaging: The effects of age, training, and coronary artery disease. Br Heart J. 1989;62(2):90-96. doi:10.1136/ hrt.62.2.90

14. Patel R, Sweeting MJ, Powell JT, Greenhalgh RM. Endovascular versus open repair of abdominal aortic aneurysm in 15-years' follow-up of the UK endovascular aneurysm repair trial 1 (EVAR trial 1): a randomised controlled trial. Lancet. 2016;388(10058):2366-2374. doi:10.1016/S0140-6736(16)31135-7

15. De Bruin JL, Baas AF, Buth J, et al. Long-term outcome of open or endovascular repair of abdominal aortic aneurysm. N Engl J Med. 2010;362(20):1881-1889. doi:10.1056/NEJMoa0909499

16. Patel HJ, Williams DM, Drews JD, et al. A 20-year experience with thoracic endovascular aortic repair. Ann Surg. 2014;260(4):691-697. doi:10.1097/SLA.0000000000000930

17. Väärämäki S, Suominen V, Pimenoff G, Saarinen J, Uurto I, Salenius J. Long-term experience of endovascular repair for thoracic aortic aneurysms and dissections. Vasc Endovascular Surg. 2016;50(5):335-342. doi:10.1177/1538574416652244

18. Ranney DN, Cox ML, Yerokun BA, Benrashid E, McCann RL, Hughes GC. Long-term results of endovascular repair for descending thoracic aortic aneurysms. J Vasc Surg. 2018;67(2):363-368. doi:10.1016/j.jvs.2017.06.094 
19. Columbo JA, Kang R, Hoel AW, Kang J. A comparison of reintervention rates after endovascular aneurysm repair between the Vascular Quality Initiative registry , Medicare claims, and chart review. J Vasc Surg. 2018;69(1):7479.e6. doi:10.1016/j.jvs.2018.03.423

20. Hiratzka LF, Bakris GL, Beckman JA, et al. 2010 ACCF/AHA/AATS/ACR/ASA/SCA/SCAI/SIR/STS/SVM Guidelines for the Diagnosis and Management of Patients with Thoracic Aortic Disease. Anesth Analg. 2010;111(2):279-315. doi:10.1016/j.jacc.2010.02.010

21. Erbel R, Aboyans V, Boileau C, et al. 2014 ESC guidelines on the diagnosis and treatment of aortic diseases. Russ J Cardiol. 2015;123(7):7-72. doi:10.15829/1560-4071-2015-07-7-72

22. Riambau V, Böckler D, Brunkwall J, et al. Management of Descending Thoracic Aorta Diseases: Clinical Practice Guidelines of the European Society for Vascular Surgery (ESVS). Eur J Vasc Endovasc Surg. 2017;53(1):4-52. doi:10.1016/j.ejvs.2016.06.005

23. van Bakel TM, de Beaufort HW, Trimarchi S, et al. Status of branched endovascular aortic arch repair. Ann Cardiothorac Surg. 2018;7(3):409-416. doi:10.21037/acs.2018.03.13

24. Rizvi AZ, Murad MH, Fairman RM, Erwin PJ, Montori VM. The effect of left subclavian artery coverage on morbidity and mortality in patients undergoing endovascular thoracic aortic interventions: A systematic review and meta-analysis. J Vasc Surg. 2009;50(5):1159-1169. doi:10.1016/j.jvs.2009.09.002

25. Delafontaine JL, Hu B, Tan TW, et al. Outcome Comparison of TEVAR with and without Left Subclavian Artery Revascularization from Analysis of Nationwide Inpatient Sample Database. Ann Vasc Surg. 2019;58(March):174-179. doi:10.1016/j.avsg.2019.01.005

26. Belvroy VM, de Beaufort HWL, van Herwaarden JA, Trimarchi S, Moll FL, Bismuth J. Type 1b Endoleaks After Thoracic Endovascular Aortic Repair are Inadequately Reported: A Systematic Review. Ann Vasc Surg. 2019. doi:10.1016/j.avsg.2019.06.030

27. Belvroy VM, De Beaufort HWL, Van Herwaarden JA, Bismuth J, Moll FL, Trimarchi S. Tortuosity of the descending thoracic aorta: Normal values by age. PLoS One. 2019;14(4):1-12. doi:10.1371/journal.pone.0215549

28. Ongstad SB, Miller DF, Panneton JM. The use of EndoAnchors to rescue complicated TEVAR procedures. $J$ Cardiovasc Surg (Torino). 2016;57(5):716-729. 


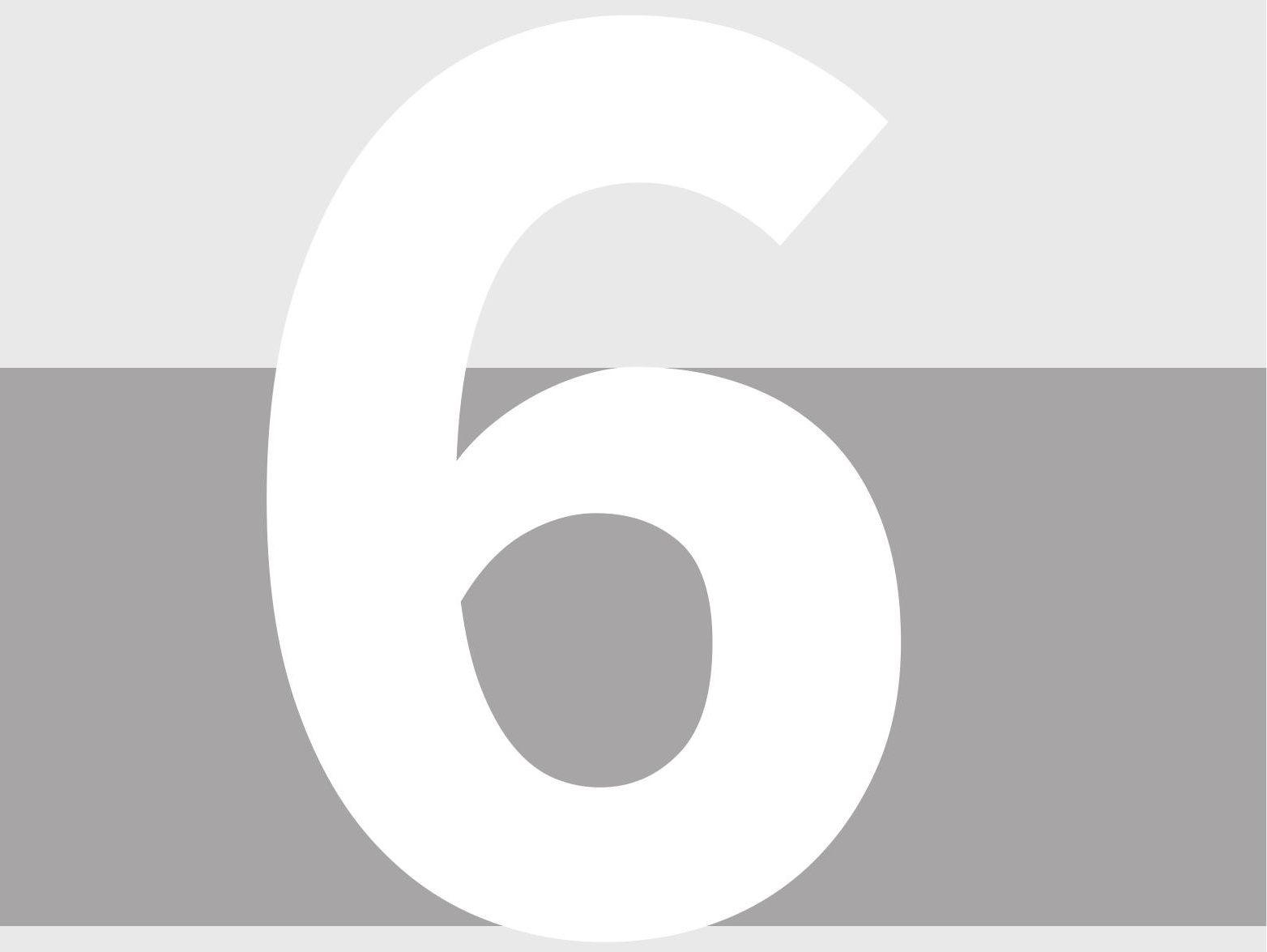




\section{Type B intramural hematoma: TEVAR or conservative approach?}

Ignas B. Houben, ${ }^{1,2}$ MD

Theodorus M.J. van Bakel, ${ }^{2,3}$ MD, PhD

Himanshu J. Patel, ${ }^{1}$ MD

${ }^{1}$ Department of Cardiac Surgery, Frankel Cardiovascular Center, University of Michigan, Ann Arbor, United States ${ }^{2}$ Department of Vascular Surgery, Utrecht University, Utrecht, The Netherlands

${ }^{3}$ Department of Vascular Surgery, Frankel Cardiovascular Center, University of Michigan, Ann Arbor, United States

Ann Cardiothorac Surg. 2019;8(4):483-487.

doi: $10.21037 / a c s .2019 .05 .18$ 



\section{INTRODUCTION}

Intramural hematoma (IMH) is the second most common presentation of acute aortic syndrome, with an estimated occurrence of $1.98 / 100,000$ person-years. ${ }^{1}$ In the majority of cases, IMH is located in the descending thoracic aorta. The clinical symptoms are similar to those of aortic dissection (AD), yet the population is older, the morphology is different, and the mortality rate is lower compared to AD.

IMH was first described by Krukenberg in $1920 .^{2}$ Ever since, the pathophysiology of the disease is yet to be elucidated. We currently have only retrospective series available to describe the natural history. This is partly due to relatively low incidence of the disease itself. The onset of IMH has been associated with the occurrence of intimal micro-tears, vasa-vasorum hemorrhage, and undiscovered penetrating atherosclerotic ulcer (PAU). ${ }^{3,4}$

\section{Wolf in sheep's clothing?}

Treatment of type B IMH starts with medical management with control of blood pressure. Operative management is reserved for patients who present with complications such as frank rupture, visceral malperfusion, intreatable pain or other signs of impending aortic rupture. The majority of these complicated cases can be managed with thoracic endovascular aortic repair (TEVAR), with recent studies describing better outcomes compared to open surgical repair. ${ }^{5,6}$ The early-mortality rate of patients treated with open vs endovascular repair has been shown to be $16 \%$ vs $5 \%$, and the 3 -year mortality rate was $23 \%$ vs. $7 \%{ }^{7}$

Clinical decision-making for uncomplicated type $\mathrm{B}$ IMH is more complex, as patients with uncomplicated IMH have either complete regression during follow-up, or may develop adverse aortic remodeling or progression into classic AD. In nonresolving IMH, the most adverse events happen in the first year after onset. ${ }^{8}$ For now, the best radiographic predictor for adverse events in uncomplicated cases is the outer diameter of the aortic wall, similar to other acute aortic syndromes. ${ }^{9} \mathrm{IMH}$-specific predictors of adverse events include the presence of ulcer like projections (ULP) and local wall thickness $>11$ $\mathrm{mm}$ (Figure 1). ${ }^{5,10,11}$ The latter predictor, however, was found in a study from 2003 that analyzed a cohort of 25 patients with type $\mathrm{A} I M \mathrm{MH}^{10}{ }^{10}$ The nomenclature in the literature may be confusing, as IMH and PAU can be considered points on a spectrum of intramural aortic pathologies. Originally PAU was described as a pathologic diagnosis. ${ }^{12}$ Contemporary clinical diagnosis is based upon computed tomography (CT) and therefore the term ulcer like projection (ULP) better reflects radiographic diagnosis. For IMH treatment, it is important to understand the etiologic differences of occurring ULPS. As described previously by Williams at el. the branch artery pseudoaneurysm may be benign and could be treated with $\mathrm{OMT}^{13}$ (Figure 2). Acute focal intimal disruptions, whether or not due to 
atherosclerotic ulcers, should be considered for TEVAR. ${ }^{14,15}$ Subacute or chronic focal intimal disruptions may be treated more conservatively. ${ }^{14}$ If CT quality allows, tiny intimal disruptions $(<3 \mathrm{~mm}$ ) should be distinguished from focal intimal disruptions ( $>3 \mathrm{~mm}$ ), as the risk for adverse events differ significantly. ${ }^{16}$ An IMH with tiny intimal disruption should, however, be followed closely in the first year after onset as they may progress into focal intimal disruptions. ${ }^{16}$

Figure 1. Radiographic IMH predicting factors for adverse events
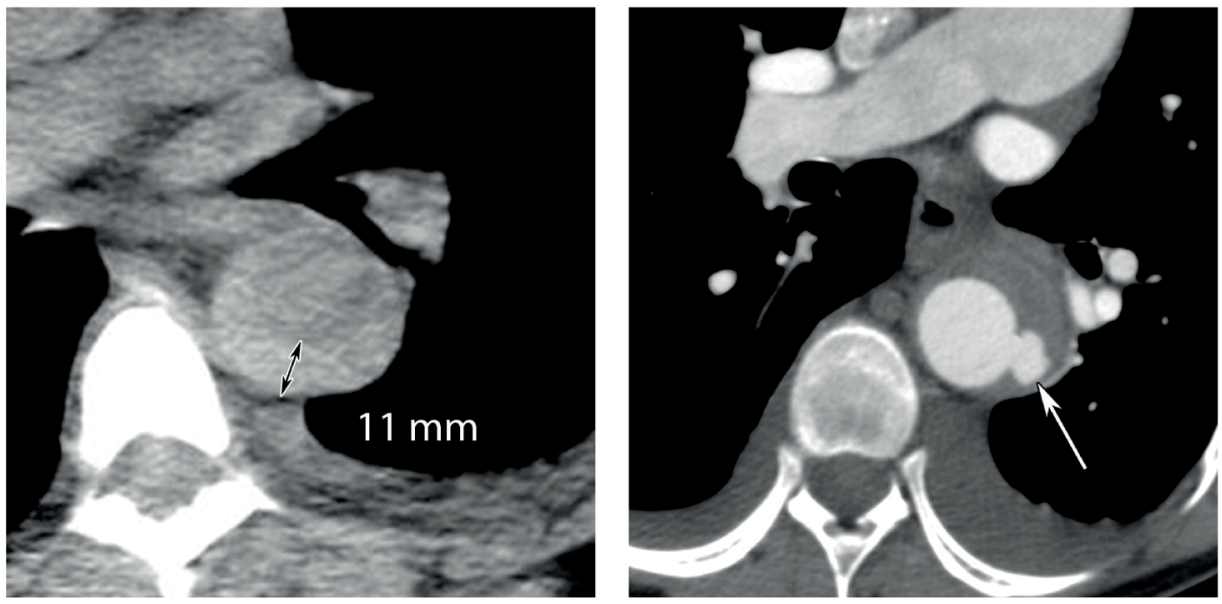

IMH predicting factors for adverse events a) Axial nonenhanced CT image shows an IMH intramural hematoma thickness of $11 \mathrm{~mm}$ (double-headed arrow). The patient is at increased risk for progression and mortality. b) Axial contrast-enhanced CT image shows an ulcer like projection (arrow) with localized contrast enhancement extending from the aortic lumen into the IMH intramural hematoma and a visible communication. Reprinted with permission from Gutschow et al. ${ }^{3}$

Figure 2. Schematic representation of aortic cross section in different pathology
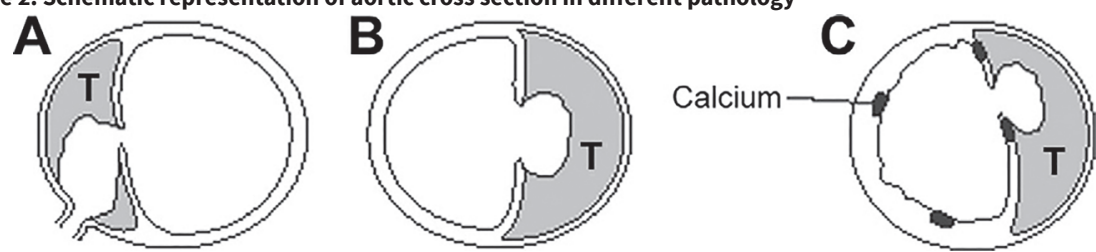

Schematic representation of aortic cross section in different pathology. As these diagrams show, the radiographic signs of ulcer like projection can encompass three potential pathologic entities. (A) The first represents a typical branch artery pseudoaneurysm, the natural history of which is benign. ( $T=$ thrombus.) (B) The middle schema shows a reentry tear developing in intramural hematoma (IMH), with local excavation of the mural thrombus. As the hematoma resolves, it can be left with an appearance of a penetrating ulcer. The diagnosis of entry tear would have been possible only if the original imaging study, in which the IMH was "fresh," was available. (C) The final schema represents the original PAU as described by Stanson et al. ${ }^{12}$ Reprinted with permission from Williams et al. ${ }^{13}$

Schematic representation of aortic cross section in different pathology. As these diagrams show, the radiographic signs of ulcer like projection can encompass three potential pathologic entities. (A) The first represents a typical branch artery pseudoa- 
neurysm, the natural history of which is benign. ( $T=$ thrombus.) (B) The middle schema shows a reentry tear developing in intramural hematoma (IMH), with local excavation of the mural thrombus. As the hematoma resolves, it can be left with an appearance of a penetrating ulcer. The diagnosis of entry tear would have been possible only if the original imaging study, in which the IMH was "fresh," was available. (C) The final schema represents the original PAU as described by Stanson et al. ${ }^{12}$ Reprinted with permission from Williams et al. ${ }^{13}$

\section{TEVAR challenges}

Endovascular repair of type $B$ IMH poses challenges similar to those that are encountered when treating type $B A D \cdot{ }^{17}$ As the mechanical properties of the IMH change during follow-up, timing of TEVAR has implications on the environment in which the stent-graft is deployed. In the acute phase, the hematoma expands and the aortic lumen is compromised most. During follow-up, the hematoma thromboses and resolves, increasing the aortic lumen over time. ${ }^{18}$ This makes stent-graft sizing challenging. In the acute phase, the stent-graft can potentially be undersized, increasing the risk of stent-graft migration and type 1 endoleak. Measuring the total aortic radius including the $\mathrm{IMH}$, on the other hand, comes with the risk of excessive oversizing and related complications. The elevated stress distributions across the wall may cause collapse of the stent-graft (Figure 3). Furthermore, apical bare metal stents and excessive balloon dilation can induce new entry-tears in friable aortic tissue. ${ }^{6,19}$ Therefore, if the aortic dimension is not increasing over time, delaying treatment is advised. ${ }^{15}$ After the subacute phase, the intimal layer stiffens and favorable aortic remodeling can be harder to achieve. ${ }^{20}$ In this phase, stentgraft sizing will rely more on the true lumen diameter, as the stiffened intima may not allow much radial oversizing.

The adverse aortic remodeling seen with older patients, can result in angulation and tortuosity of the diseased aorta, which increases hemodynamic displacement forces acting on the surface of the stent-graft. ${ }^{21}$ In this anatomic configuration, an extended length of repair is often necessary to achieve appropriate sealing in the proximal and distal landing zones. ${ }^{21,22}$ Extended repair also increases the risks of stroke, spinal cord ischemia and type 3 endoleak at the intermodular junctions. ${ }^{23,24}$ Finally, current stent-grafts for TEVAR are 50-200 times stiffer than healthy aortic tissue..$^{25,26}$ This increased stiffness of the aorta following stent-graft deployment can increase stresses in the untreated segments of the aortic wall. ${ }^{27}$ Furthermore, left ventricular stroke work is estimated to increase up to $26 \%$ as a consequence of stent-graft induced descending aortic stiffening, resulting in adverse left ventricular remodeling. ${ }^{28}$ 
Figure 3. Biomechanical features of stent grafting in descending thoracic aortic pathology

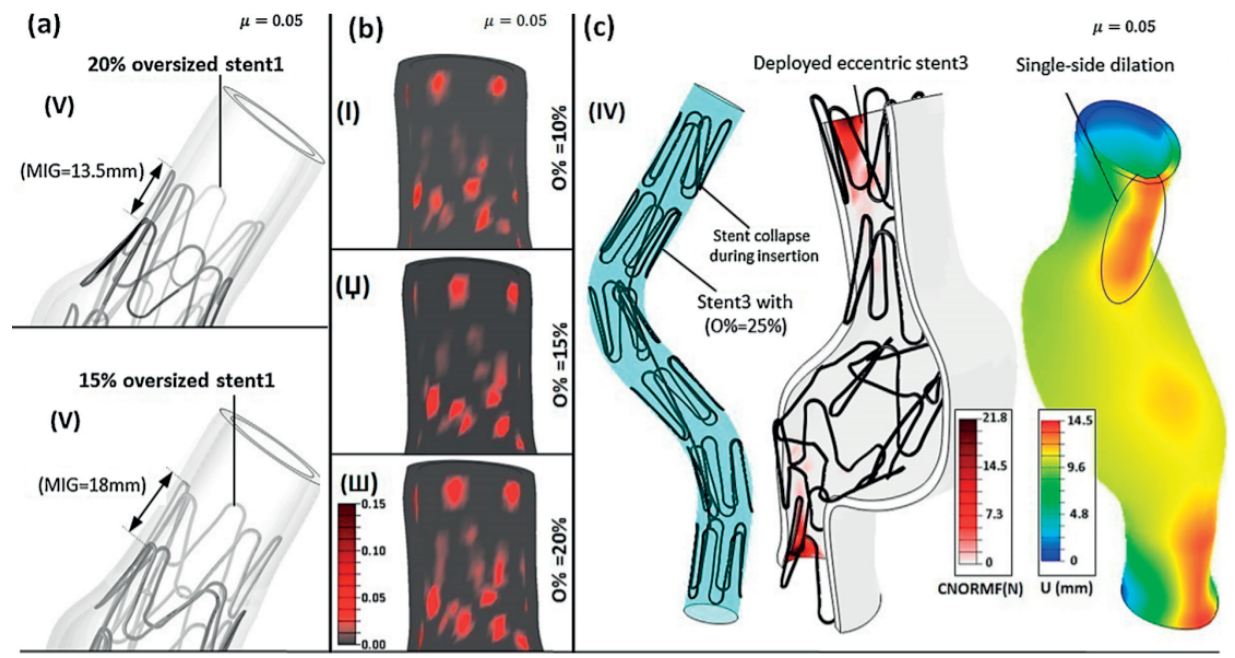

a) Impact of $20 \%$ oversizing on proximal landing zone stent migration. (b) Contact pressure stresses (MPa) in the proximal landing zone for different percentages of oversizing. (c) Excessive oversizing with stent-graft collapse and increased dilatation $(\mathrm{U})$ in the landing zones. Reprinted with permission from Altnji et al. ${ }^{29} \mathrm{MIG}=$ migration, $\mathrm{O} \%$ = percentage of oversizing, $\mathrm{F}=$ force (Newton), $\mathrm{U}=$ displacement (millimeter).

\section{CONCLUSION}

Uncomplicated type B IMH optimally treated with medical management. TEVAR is reserved for patients with complications and requires an experienced surgeon who can identify patient-specific challenges that increase the risk of the procedure. Factors such as the presence of ULP should be taken into consideration for the decision to operate and reduce the long-term risk of aortic rupture.

However, large prospective or retrospective cohort data, generating IMH specific outcomes are lacking. Contemporary decision-making is based on: 1) retrospective cohorts from >10 years ago comprising no more than 66 patients with isolated IMHs; 2) clinical experience; and 3) acute aortic syndrome reports with a majority describing classic double barrel aortic dissections.

Further research is necessary and should focus on providing a stratified risk model coupled with a natural history assessment in a large cohort and should be contrasted to that seen with classic aortic dissection. 
Type B intramural hematoma: TEVAR or conservative approach?

\section{REFERENCES}

1. DeMartino RR, Sen I, Huang Y, et al. Population-Based Assessment of the Incidence of Aortic Dissection, Intramural Hematoma, and Penetrating Ulcer, and Its Associated Mortality From 1995 to 2015. Circ Cardiovasc Qual Outcomes. 2018;11(8). doi:10.1161/CIRCOUTCOMES.118.004689

2. Krukenberg E. Contribution to the question of dissecting aneurysm. Beitr Pathol Anat Allg Pathol. 1920;67:32951.

3. Gutschow SE, Walker CM, Martínez-Jiménez S, Rosado-de-Christenson ML, Stowell J, Kunin JR. Emerging Concepts in Intramural Hematoma Imaging. RadioGraphics. 2016;36(3):660-674. doi:10.1148/rg.2016150094

4. Park KH, Lim C, Choi JH, et al. Prevalence of Aortic Intimal Defect in Surgically Treated Acute Type A Intramural Hematoma. Ann Thorac Surg. 2008;86(5):1494-1500. doi:10.1016/j.athoracsur.2008.06.061

5. Ganaha F, Miller DC, Sugimoto K, et al. Prognosis of aortic intramural hematoma with and without penetrating atherosclerotic ulcer: A clinical and radiological analysis. Circulation. 2002;106(3):342-348. doi:10.1161/01. CIR.0000022164.26075.5A

6. Patel HJ, Williams DM, Upchurch GR, Dasika NL, Deeb GM. The challenge of associated intramural hematoma with endovascular repair for penetrating ulcers of the descending thoracic aorta. J Vasc Surg. 2010;51(4):829835. doi:10.1016/j.jvs.2009.11.050

7. Evangelista A, Czerny M, Nienaber C, et al. Interdisciplinary expert consensus on management of type B intramural haematoma and penetrating aortic ulcer. Eur J Cardio-Thoracic Surg. 2015;47(2):209-217. doi:10.1093/ ejcts/ezu386

8. Sailer AM, Nelemans PJ, Hastie TJ, et al. Prognostic significance of early aortic remodeling in acute uncomplicated type B aortic dissection and intramural hematoma. J Thorac Cardiovasc Surg. 2017;154:1192-1200. doi:10.1016/j.jtcvs.2017.04.064

9. Writing Committee, Riambau V, Böckler D, et al. Editor's Choice - Management of Descending Thoracic Aorta Diseases: Clinical Practice Guidelines of the European Society for Vascular Surgery (ESVS). Eur J Vasc EndovasC Surg. 2017;53(1):4-52. doi:10.1016/j.ejvs.2016.06.005

10. Song J-M, Kim H-S, Song J-K, et al. Usefulness of the initial noninvasive imaging study to predict the adverse outcomes in the medical treatment of acute type A aortic intramural hematoma. Circulation. 2003;108 Suppl 1(90101):II324-8. doi:10.1161/01.cir.0000087651.30078.38

11. Von Kodolitsch Y, Csösz SK, Koschyk DH, et al. Intramural Hematoma of the Aorta Predictors of Progression to Dissection and Rupture. Circulation. 2003;107:1158-1163. doi:10.1161/01.CIR.0000052628.77047.EA

12. Stanson AW, Kazmier FJ, Hollier LH, et al. Penetrating atherosclerotic ulcers of the thoracic aorta : natural history and clinicopathologic correlations. Ann Vasc Surg. 1986;1(1):15-23. doi:10.1016/S0890-5096(06)60697-3

13. Williams DM, Cronin P, Dasika N, et al. Aortic Branch Artery Pseudoaneurysms Accompanying Aortic Dissection. Part II. Distinction from Penetrating Atherosclerotic Ulcers. J Vasc Interv Radiol. 2006;17(5):773-781. doi:10.1097/01.RVI.0000209345.00529.DF

14. Moral S, Cuéllar H, Avegliano G, et al. Clinical Implications of Focal Intimal Disruption in Patients With Type B Intramural Hematoma. J Am Coll Cardiol. 2017;69(1):28-39. doi:10.1016/j.jacc.2016.10.045

15. Patel HJ, Sood V, Williams DM, Dasika NL, Diener AC, Deeb GM. Late outcomes with repair of penetrating thoracic aortic ulcers: The merits of an endovascular approach. Ann Thorac Surg. 2012;94(2):516-523. doi:10.1016/j. athoracsur.2012.03.074

16. Moral S, Ballesteros E, Roque M, et al. Intimal disruption in type B aortic intramural hematoma. Does size matter? A systematic review and meta-analysis. Int J Cardiol. 2018;269:298-303. doi:10.1016/j.ijcard.2018.07.111

17. van Bakel TMJ, Figueroa CA, van Herwaarden JA, Trimarchi S. Challenges of Thoracic Endovascular Aortic Repair for Type B Aortic Dissection. J Endovasc Ther. 2018;25(5):578-580. doi:10.1177/1526602818795153

18. Evangelista A, Dominguez R, Sebastia C, et al. Long-term follow-up of aortic intramural hematoma: Predictors of outcome. Circulation. 2003;108(5):583-589. doi:10.1161/01.CIR.0000081776.49923.5A

19. Jánosi RA, Gorla R, Tsagakis K, et al. Thoracic endovascular repair of complicated penetrating aortic ulcer: An 11-year single-center experience. J Endovasc Ther. 2016;23(1):150-159. doi:10.1177/1526602815613790 
Current status of descending thoracic aortic assessment

20. Peterss S, Ross JA, Elefteriades JA, et al. Changing Pathology of the Thoracic Aorta From Acute to Chronic Dissection. J Am Coll Cardiol. 2016;68(10):1054-65. doi:10.1016/j.jacc.2016.05.091

21. Figueroa CA, Taylor CA, Chiou AJ, Yeh V, Zarins CK. Magnitude and direction of pulsatile displacement forces acting on thoracic aortic endografts. J Endovasc Ther. 2009;16(3):350-358. doi:10.1583/09-2738.1

22. Kasirajan K, Milner R, Chaikof EL. Late complications of thoracic endografts. J Vasc Surg. 2006;43(2):A94-A99. doi:10.1016/j.jvs.2005.10.064

23. Prasad A, To LK, Gorrepati ML, Zarins CK, Figueroa CA. Computational analysis of stresses acting on intermodular junctions in thoracic aortic endografts. J Endovasc Ther. 2011;18(4):559-568. doi:10.1583/11-3472.1

24. Gutsche JT, Cheung AT, McGarvey ML, et al. Risk factors for perioperative stroke after thoracic endovascular aortic repair. Ann Thorac Surg. 2007;84(4):1195-1200; discussion 1200. doi:10.1016/j.athoracsur.2007.04.128

25. Santos IC, Rodrigues A, Rocha LA, Tavares JMR, Figueiredo L, Santos IC. Mechanical properties of stent-graft materials. Proc Inst Mech Eng Part L J Mater Des Appl. 2012;226(4):330-341. doi:10.1177/1464420712451065

26. De Beaufort HWL, Coda M, Conti M, et al. Changes in aortic pulse wave velocity of four thoracic aortic stent grafts in an ex vivo porcine model. PLoS One. 2017;12(10). doi:10.1371/journal.pone.0186080

27. van Bakel TMJ, Burris NS, Patel HJ, Figueroa CA. Ascending aortic rupture after zone 2 endovascular repair: a multiparametric computational analysis. Eur J Cardio-Thoracic Surg. 2019;0:1-4. doi:10.1093/ejcts/ezy458

28. van Bakel TM, Arthurs CJ, Nauta FJ, et al. Cardiac remodeling following thoracic endovascular aortic repair for descending aortic aneurysms. Eur J Cardio-Thoracic Surg. 2018;0:1-10. doi:10.1016/0038-1098(79)91043-3

29. Altnji HE, Bou-Said B, Walter-Le Berre H. Morphological and stent design risk factors to prevent migration phenomena for a thoracic aneurysm: a numerical analysis. Med Eng Phys. 2015;37(1):23-33. doi:10.1016/j. medengphy.2014.09.017 



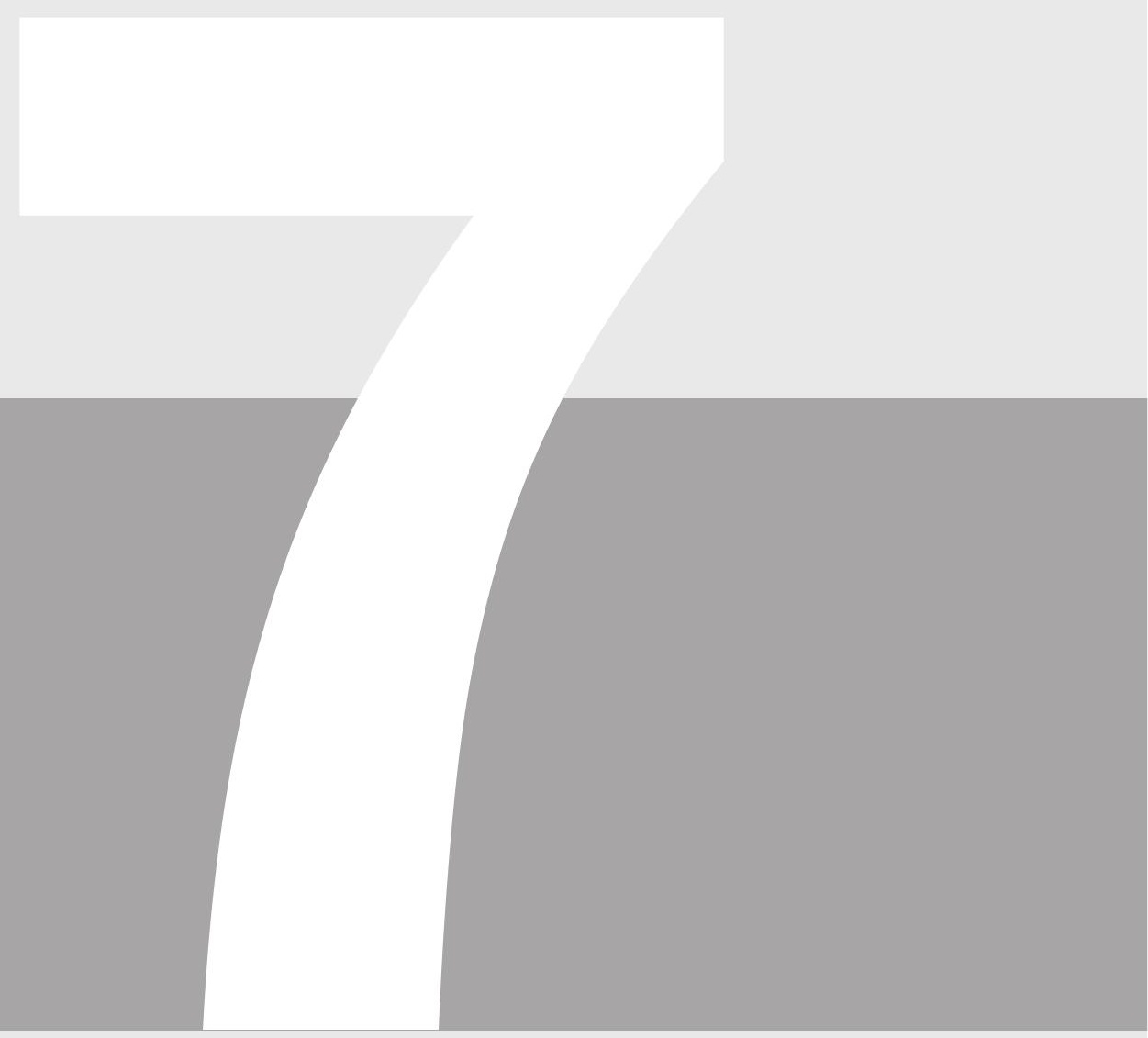




\section{Thoracic endovascular aortic repair in the setting of compromised distal landing zones}

Ignas B. Houben, MD, ${ }^{1,4}$

Byron G. DuBois, MD, ${ }^{2}$

Minhaj S. Khaja, MD, MBA, ${ }^{2,3}$

Bo Yang, MD, PhD, ${ }^{1}$

Karen M. Kim, MD, MS, ${ }^{1}$

Joost A. van Herwaarden, MD, PhD, ${ }^{4}$

David M. Williams, MD, ${ }^{2}$

Himanshu J. Patel, MD ${ }^{1}$

${ }^{1}$ Department of Cardiac Surgery, Frankel Cardiovascular Center, University of Michigan, Ann Arbor, United States ${ }^{2}$ Department of Radiology, University of Michigan, Ann Arbor, United States

${ }^{3}$ Department of Radiology, University of Virginia Medical Center, Charlottesville, Virginia, United States

${ }^{4}$ Department of Vascular Surgery, Utrecht University, Utrecht, The Netherlands 


\section{ABSTRACT}

\section{Purpose}

To report short- and intermediate-term outcomes of two distinct approaches to thoracic endovascular aortic repair (TEVAR) for descending aortic aneurysms in patients with compromised distal landing zones (CDLZs).

\section{Methods}

51 patients (38 female, average age $72 \pm 9$ years) underwent 55 TEVARs (2008-2018) for aneurysmal disease. Inclusion criteria consisted of TEVAR in a CDLZ, defined as: $\geq 3.5 \mathrm{~cm}$ diameter, $\geq 50 \%$ cross-sectional thrombus, or $\geq 25 \%$ circumferential mural calcification in the $2 \mathrm{~cm}$ supraceliac aorta, or tortuosity index $\geq 1.1$ over the $10 \mathrm{~cm}$ supraceliac aorta. Treatment cohorts were: 1 ) TEVAR alone ( $n=29)$; 2 ) TEVAR with adjunct consisting of visceral snorkel graft with distal stent extension $(n=20)$ and/or EndoAnchors $(n=6)$.

\section{Results}

Perioperative complication rate was 20\%. 30-day mortality was 5\% including 1 accesssite related intraoperative death and 1 postoperative death from embolic mesenteric ischemia. Median clinical follow-up was 2.2 years. Intermediate-term outcomes include: type 1B endoleaks, 35\%; $\geq 0.5 \mathrm{~cm} /$ year maximal aortic diameter growth, $9 \%$; reintervention, 15\%; and all-cause mortality, 25\%. The distal landing zone diameter increased by $0.3 \mathrm{~cm} /$ year in the TEVAR alone however decreased by $0.1 \mathrm{~cm} /$ year in the adjunct cohort $(p=0.04)$.

\section{Conclusion}

TEVAR is a viable alternative for the treatment of thoracoabdominal aortic aneurysms in patients with CDLZs, although these patients may benefit significantly from the development of branched thoracoabdominal devices. In the interim, the use of TEVAR adjuncts may limit progressive degeneration of the distal landing zone in this patient population. 


\section{INTRODUCTION}

Thoracic endovascular aortic repair (TEVAR) has become the mainstay of aortic intervention for descending thoracic aortic pathology. ${ }^{1-4}$ Its durability hinges on achieving adequate seal in the endograft landing zones (LZs), with the distal LZ largely understudied. ${ }^{5,6}$ Only recently are suboptimal distal LZs receiving attention for their role in major complications including stent graft migration, type $1 \mathrm{~b}$ endoleaks, and distal stent graftinduced new entry tears, all of which can contribute to further aortic degeneration. ${ }^{7,8}$

Currently available Food and Drug Administration-approved endografts recommend a minimum $2 \mathrm{~cm}$ LZ proximal to the celiac axis to ensure adequate seal. The limitation makes the subset of patients with compromised distal landing zones (CDLZs) who are poor surgical candidates a unique therapeutic challenge. In the absence of an ideal endovascular option, this study reports the short- and intermediate-term outcomes of two distinct treatment modalities (TEVAR alone or with adjunct) for the management of thoracoabdominal aortic aneurysms in patients with CDLZs.

\section{METHODS}

\section{Patient data}

This is a single-center retrospective review of data from consecutive, prospectively identified patients with CDLZs who underwent endovascular thoracoabdominal aortic repair at the University of Michigan Health System between June 2008 and November 2018. Relevant data was obtained from the electronic medical record and Society of Thoracic Surgeons (STS) National Database. The study was conducted with Institutional Review Board approval (HUM00166977, date of approval 8/13/2019) and in compliance with the Health Insurance Portability and Accountability Act. Informed consent was not required due to its retrospective nature.

Inclusion criteria consist of endovascular repair of thoracoabdominal aortic aneurysm and presence of CDLZ, as determined by a senior cardiothoracic surgeon and interventional radiologist with greater than 50 years of combined experience. CDLZ was defined as presence of at least one of the following: $\geq 3.5 \mathrm{~cm}$ diameter, $\geq 50 \%$ cross-sectional thrombus, or $\geq 25 \%$ circumferential mural calcification in the $2 \mathrm{~cm}$ supraceliac aorta, or tortuosity index $\geq 1.1$ over the $10 \mathrm{~cm}$ supraceliac aorta (Figure 1). ${ }^{8}$ Patients with aortic dissection pathology were excluded $(n=18)$.

Patient demographics and comorbidities are summarized in Table $1 .{ }^{9}$ A total of 55 TEVARs were attempted in 51 patients with CDLZs, with 4 patients undergoing TEVAR 
Figure 1: Proposed investigational criteria that define a 'compromised' distal landing zone

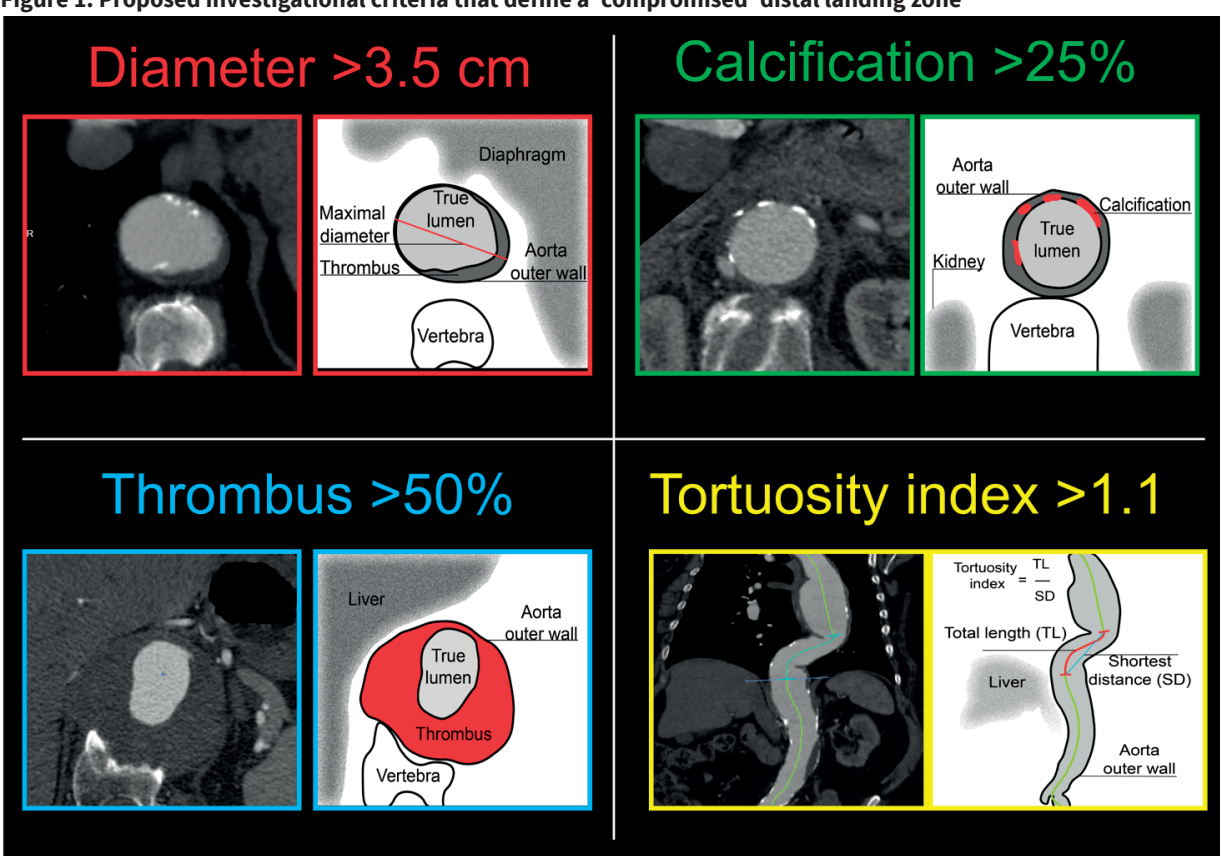

extension. 29 (53\%) cases involved TEVAR alone and 26 (47\%) TEVAR with adjunct which were divided into visceral snorkel graft with distal stent extension $(n=20)$ and/or Aptus Heli-FX EndoAnchors $(n=6)$ (Medtronic; Minneapolis, MN). ${ }^{10}$

Primary outcomes were presence of type 1B endoleak, maximal aortic diameter growth, freedom from reintervention, secondary surgical conversion, and mortality. ${ }^{11}$ Secondary outcomes included intraoperative fluoroscopy time, major complication rate, postoperative length of stay, stent graft wedge apposition, ${ }^{8}$ and distal stent migration. Patients were classified by endovascular treatment modality. Primary analysis was performed on the entire patient population as well as the two treatment cohorts: 1) TEVAR and 2) TEVAR with adjunct (snorkel with distal stent extension and/or EndoAnchor).

\section{Aortic imaging and measurements}

Computed tomography angiography (CTA) was performed on a multi-slice scanner after intravenous injection of 120 mL Isoview-370 contrast (Bracco Diagnostics; Milan, Italy) with prospective multiplanar reconstructions performed in the mid-diastolic phase (75\% of the R-R interval). The images were analyzed using Vitrea Core (Vital Images Inc., Product Version 6.9.1; Minnetonka, MN). 
Table 1. Baseline patient characteristics

\begin{tabular}{|c|c|c|c|c|}
\hline & $\begin{array}{c}\text { Total } \\
(n=55)\end{array}$ & $\begin{array}{l}\text { TEVAR } \\
(n=29)\end{array}$ & $\begin{array}{c}\text { TEVAR with } \\
\text { adjunct }(n=26)\end{array}$ & $\mathrm{p}$-value \\
\hline Age (years) & $73 \pm 8$ & $73 \pm 6$ & $72 \pm 11$ & 0.53 \\
\hline Female & $38(69)$ & $19(65)$ & $19(73)$ & 0.55 \\
\hline \multicolumn{5}{|l|}{ Comorbidities } \\
\hline Hypertension & $53(96)$ & $28(97)$ & $25(96)$ & 0.94 \\
\hline Hyperlipidemia & $49(89)$ & $26(90)$ & $23(88)$ & 0.89 \\
\hline Diabetes mellitus & $10(55)$ & $7(24)$ & $3(12)$ & 0.23 \\
\hline Creatinine $\geq 1.5 \mathrm{mg} / \mathrm{dL}$ & $13(23)$ & $6(21)$ & $7(27)$ & 0.59 \\
\hline Hemodialysis dependent & $2(4)$ & $1(3)$ & $1(4)$ & 0.94 \\
\hline Peripheral vascular disease & $26(55)$ & $14(48)$ & $12(46)$ & 0.88 \\
\hline Arrhythmia & $7(13)$ & $2(7)$ & $5(19)$ & 0.17 \\
\hline Coronary artery disease & $16(29)$ & $9(31)$ & $7(27)$ & 0.68 \\
\hline Congestive heart failure & $7(13)$ & $4(14)$ & $3(12)$ & 0.80 \\
\hline LV ejection fraction $\leq 40 \%{ }^{a}$ & $3(6)$ & $2(7)$ & $1(5)$ & 0.72 \\
\hline History of CVA & $11(20)$ & $8(28)$ & $3(12)$ & 0.14 \\
\hline Tobacco history & $40(73)$ & $23(79)$ & $17(65)$ & 0.25 \\
\hline COPD & $21(38)$ & $12(41)$ & $9(35)$ & 0.61 \\
\hline FEV1 $\leq 60 \%$ predicted $^{\mathrm{b}}$ & $8(20)$ & $5(25)$ & $3(15)$ & 0.43 \\
\hline Connective tissue disorder & $4(7)$ & $1(3)$ & $3(12)$ & 0.25 \\
\hline Family history of aortic aneurysm & $12(22)$ & $9(31)$ & $3(12)$ & 0.08 \\
\hline ASAPS $^{\circledR}$ Score 4 & $36(65)$ & $16(55)$ & $20(77)$ & 0.09 \\
\hline Maximal descending aortic diameter $(\mathrm{cm})$ & $6.6 \pm 1.3$ & $6.4 \pm 1.1$ & $6.8 \pm 1.4$ & 0.52 \\
\hline Prior aortic repair & $31(56)$ & $14(29)$ & $17(65)$ & 0.20 \\
\hline Open surgery ${ }^{c}$ & $24(77)$ & $13(93)$ & $11(65)$ & 0.85 \\
\hline Endovascular $^{d}$ & $14(45)$ & $2(14)$ & $12(71)$ & $<0.001$ \\
\hline Era of procedure & & & & 0.02 \\
\hline $2008-2012$ & $17(31)$ & $13(45)$ & $4(15)$ & \\
\hline $2013-2018$ & $38(69)$ & $16(55)$ & $22(85)$ & \\
\hline Acuity of repair & & & & 0.24 \\
\hline Emergent & $2(4)$ & $0(0)$ & $2(8)$ & \\
\hline Urgent & $3(5)$ & $1(3)$ & $2(8)$ & \\
\hline
\end{tabular}

${ }^{a}$ Out of 51 cases with preoperative echocardiograms

${ }^{\mathrm{b}}$ Out of 40 cases with preoperative pulmonary function tests

'Anesthesiology Society of America Physical Status Classification System ${ }^{9}$

${ }^{\mathrm{d} O u t}$ of 31 cases with at least one prior aortic repair

Following centerline analysis, axial slices of the aorta were evaluated at four levels relative to the rostral celiac ostium (Figure 2): 1) $2 \mathrm{~cm}$ proximal; 2) at the rostral celiac ostium; 3) $1 \mathrm{~cm}$ distal; 4) $2 \mathrm{~cm}$ distal. The following measurements were taken: 1) outer wall cross-sectional area; 2) maximal outer wall diameter; 3) patent true lumen cross- 
sectional area (excluding intraluminal thrombus). Percent cross-sectional thrombus was defined as the difference between the outer wall and patent true lumen cross-sectional areas, divided by the former. Percent circumferential mural calcification and tortuosity index of the $10 \mathrm{~cm}$ supraceliac aorta were calculated for baseline pre-TEVAR CTAs.

Figure 2. Representative patient aorta with thoracoabdominal pathology.

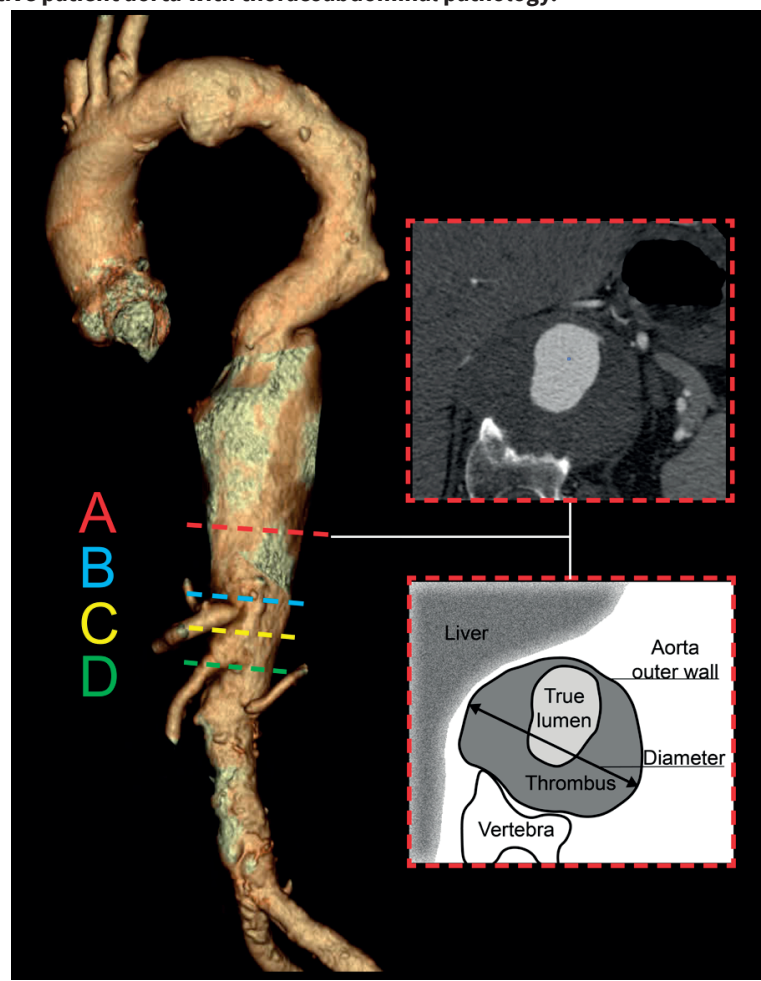

Following centerline analysis, axial slices were evaluated at four levels relative to the rostral celiac ostium: A) $2 \mathrm{~cm}$ proximal; B) at the rostral celiac ostium; C) $1 \mathrm{~cm}$ distal; D) $2 \mathrm{~cm}$ distal.

Additional measurements included: 1) distal stent migration, defined as distance along the aortic centerline between the distal-most aspect of the stent and the nearest visceral arterial ostium ( $\geq 1 \mathrm{~cm}$ was deemed substantial); 2 ) stent graft wedge apposition, defined as distance between planes perpendicular to the centerline that capture the proximaland distal-most aspects of the distal stent edge ( $\geq 1 \mathrm{~cm}$ was deemed substantial) (Figure $\underline{3}) ;^{8}$ 3) maximal outer wall diameter of the descending thoracic aorta as per the STS reporting standards. ${ }^{12}$ The distal LZs were evaluated for presence of type 1B endoleaks. 
Figure 3: Representative imaging from the endovascular treatment strategies

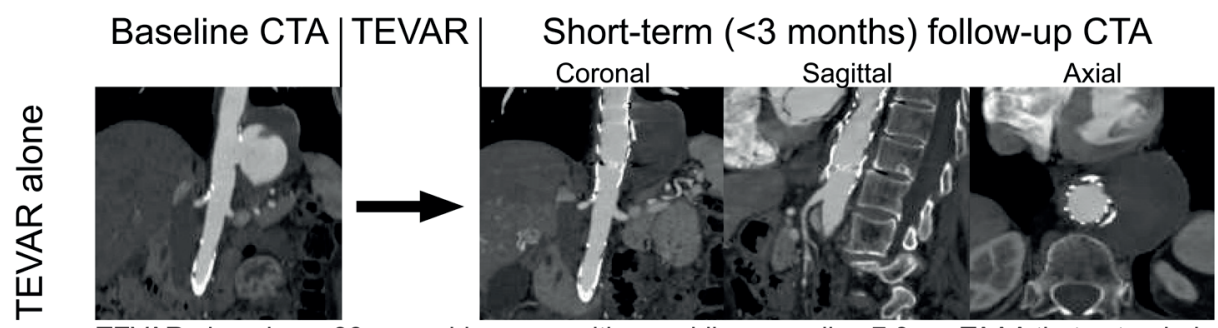

TEVAR alone in an 86-year-old woman with a rapidly expanding $7.0 \mathrm{~cm}$ TAAA that extended to the rostral celiac ostium.

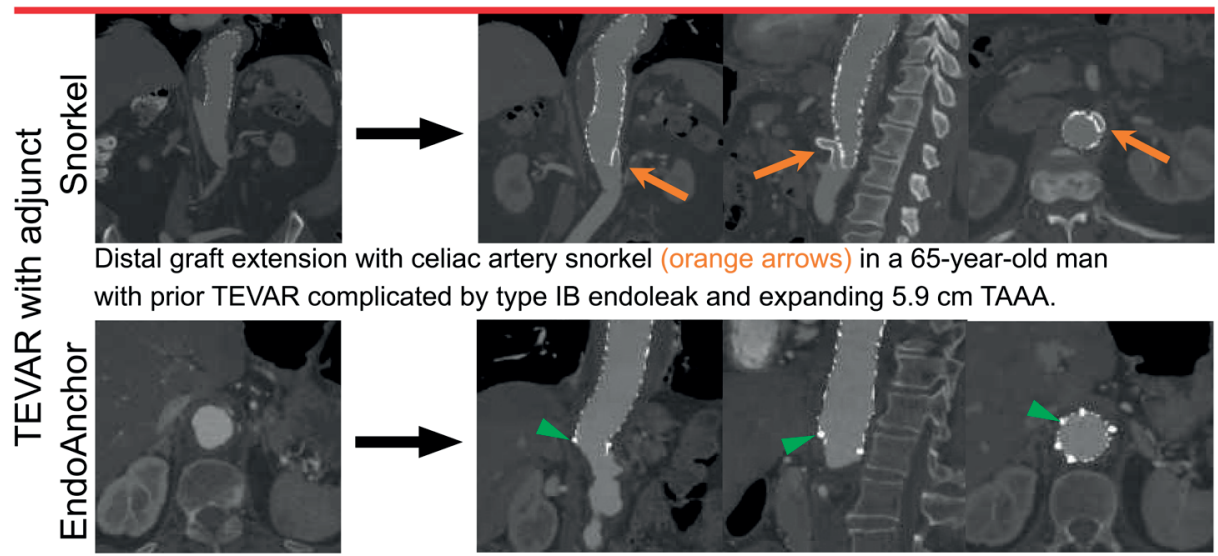

TEVAR with distal EndoAnchors (green arrowheads) in a 69-year-old woman with incidentally discovered $8.2 \mathrm{~cm}$ TAAA that extended to the rostral celiac ostium.

\section{Statistical analysis}

Continuous data were presented as mean \pm standard deviation and categorical data were presented as frequency and percentage. Variables missing more than $10 \%$ of patient data are labelled. Normal distribution of the data was determined by ShapiroWilk test. Any difference between groups was assessed using chi-square analysis for categorical data and t-test for continuous data. A multivariate linear regression analysis was performed using clinical expertise and literature review to define the confounders. Statistical significance was assumed at $p £ 0.05$. Analysis of the data was performed using SPSS Statistics 26 (IBM; Armonk, NY).

\section{Operative techniques and follow-up}

TEVARs were performed in the presence of at least one senior cardiothoracic surgeon and one senior interventional radiologist. Previously described preoperative planning, procedural technique, and follow-up schedule ${ }^{13}$ were employed with adjustments tailored to the CDLZ as follows: 
1) Treatment modality (TEVAR alone or with adjunct) was determined on a case-by-case basis, influenced by the relative extent of supra-versus periceliac aortic aneurysmal degeneration, burden of intraluminal thrombus, and accessibility of the thoracoabdominal visceral arteries.

2) Aortic stent grafts were not post-dilated in distal LZs with significant mural thrombus to mitigate the risk of distal embolization.

3) Clinically significant partial occlusion of a visceral artery by aortic stent graft was defined by mean arterial pressure gradient $\geq 10 \mathrm{~mm} \mathrm{Hg}$. If this threshold was met a snorkel stent was placed and the distal LZ extended.

4) Visceral arteries were pre-cannulated and snorkel stents placed via contralateral femoral access. A balloon was kept inflated in the snorkel while the distal-most aortic stent graft was deployed so as to prevent luminal narrowing. The length of the intra-aortic portion of the snorkel varied by target visceral artery, with celiac snorkel stents terminating near the rostral superior mesenteric artery (SMA) ostium.

5) Aptus Heli-FX EndoAnchors were deployed circumferentially at the caudal-most aspect of the distal aortic endograft when mural thrombus was absent. ${ }^{14}$ In cases of significant asymmetric mural thrombus they were deployed in the non-thrombosed quadrants.

6) Patients in the TEVAR cohort were discharged on a single antiplatelet regimen of daily acetylsalicylic acid $325 \mathrm{mg}$ for 30 days. This regimen was supplemented with daily Clopidogrel $75 \mathrm{mg}$ in patients who underwent snorkel placement.

\section{RESULTS}

\section{Baseline distal landing zone characteristics}

A total of 53 cases had available baseline CTAs with the remaining 2 cases determined to have CDLZ based on intraoperative arteriography. There were minor differences in distal LZ characteristics between cohorts attributable to technical considerations specific to the treatment modalities, summarized in Table 2.

\section{Procedural details}

Procedural parameters are summarized in Table 3. Overall technical success rate was 98\%. One patient in the TEVAR alone cohort died intraoperatively due to an access-site related complication. A total of 20 cases underwent snorkel placement, $16(80 \%)$ of which involved the celiac artery, $2(10 \%)$ the superior mesenteric artery, and $2(10 \%)$ the renal artery. 8 cases underwent Aptus Heli-FX EndoAnchor fixation of the distal seal zone, with an average of 8 EndoAnchors (range of 4-14) placed per case. 
Table 2. Baseline distal landing zone characteristics

\begin{tabular}{|c|c|c|c|c|c|}
\hline & & Total $(n=53)$ & $\operatorname{TEVAR}(n=29)$ & $\begin{array}{c}\text { TEVAR with } \\
\text { adjunct }(n=24)\end{array}$ & $\mathrm{p}$-value \\
\hline \multicolumn{6}{|c|}{ Baseline diameter $(\mathrm{cm})$} \\
\hline & $2 \mathrm{~cm}$ proximal ${ }^{a}$ & $5.0 \pm 0.9$ & $4.8 \pm 1.0$ & $5.3 \pm 0.6$ & 0.006 \\
\hline & Rostral celiac ostium & $4.3 \pm 0.8$ & $4.3 \pm 0.9$ & $4.3 \pm 0.6$ & 0.77 \\
\hline & $1 \mathrm{~cm}$ distal $^{\mathrm{a}}$ & $3.9 \pm 1.0$ & $4.0 \pm 0.9$ & $3.8 \pm 1.1$ & 0.51 \\
\hline & $2 \mathrm{~cm}$ distal $^{\mathrm{a}}$ & $3.8 \pm 8.3$ & $3.8 \pm 0.8$ & $3.7 \pm 8$ & 0.82 \\
\hline \multicolumn{6}{|c|}{ Cross-sectional thrombus $\geq 50 \%$ (n) } \\
\hline & $2 \mathrm{~cm}$ proximal ${ }^{\mathrm{a}}$ & $15(28)$ & $8(28)$ & $7(29)$ & 0.90 \\
\hline & Rostral celiac ostium & $12(23)$ & $10(34)$ & $2(8)$ & 0.02 \\
\hline & $1 \mathrm{~cm}$ distal $^{\mathrm{a}}$ & $7(13)$ & $6(21)$ & $1(4)$ & 0.08 \\
\hline & $2 \mathrm{~cm}_{\text {distal }}{ }^{\mathrm{a}}$ & $8(15)$ & $6(21)$ & $2(8)$ & 0.21 \\
\hline \multicolumn{6}{|c|}{ Mural calcification $\geq 25 \%$ (n) } \\
\hline & $2 \mathrm{~cm}$ proximal ${ }^{\mathrm{a}}$ & $1(2)$ & $0(0)$ & $1(4)$ & 0.27 \\
\hline & Rostral celiac ostium & $3(6)$ & $1(3)$ & $2(8)$ & 0.44 \\
\hline & $1 \mathrm{~cm}$ distal $^{\mathrm{a}}$ & $3(6)$ & $1(3)$ & $2(8)$ & 0.44 \\
\hline & $2 \mathrm{~cm}$ distal $^{\mathrm{a}}$ & $1(2)$ & $0(0)$ & $1(4)$ & 0.27 \\
\hline Tortuosity $\geq 1.1$ (n) & & $10(19)$ & $6(21)$ & $4(17)$ & 0.71 \\
\hline
\end{tabular}

${ }^{\mathrm{a}}$ Relative to rostral celiac ostium

Table 3. Periprocedural outcomes

\begin{tabular}{|c|c|c|c|c|}
\hline & Total $(n=55)$ & $\begin{array}{l}\text { TEVAR } \\
(n=29)\end{array}$ & $\begin{array}{l}\text { TEVAR with } \\
\text { adjunct }(n=26)\end{array}$ & $\mathrm{p}$-value \\
\hline Contrast volume (mL) & $110 \pm 43$ & $110 \pm 37$ & $100 \pm 49$ & 0.47 \\
\hline Procedure time (min) & $260 \pm 120$ & $220 \pm 94$ & $300 \pm 130$ & 0.005 \\
\hline Fluoroscopy time (min) & $56 \pm 43$ & $37 \pm 19$ & $77 \pm 51$ & $<0.001$ \\
\hline Estimated blood loss (mL) & $340 \pm 550$ & $420 \pm 660$ & $260 \pm 380$ & 0.88 \\
\hline Technical success & $54(98)$ & $28(97)$ & $26(100)$ & 0.34 \\
\hline Stent wedge apposition $\geq 1 \mathrm{~cm} \alpha$ & $30(63)$ & $16(64)$ & $14(61)$ & 0.82 \\
\hline Type 1B endoleak $\alpha$ & $8(17)$ & $6(23)$ & $2(9)$ & 0.20 \\
\hline ICU LOS (hrs) & $77 \pm 49$ & $89 \pm 63$ & $65 \pm 22$ & 0.26 \\
\hline Postoperative LOS (days) & $7 \pm 6$ & $8 \pm 7$ & $7 \pm 5$ & 0.89 \\
\hline $\begin{array}{l}\text { Unplanned additional intraoperative } \\
\text { maneuvers }\end{array}$ & $5(9)$ & $1(3)$ & $4(15)$ & 0.44 \\
\hline Major complications & $11(20)$ & $8(28)$ & $3(12)$ & 0.35 \\
\hline 30-day mortality & $3(5)$ & $2(7)$ & $1(4)$ & 0.62 \\
\hline
\end{tabular}

$\alpha$ Out of 48 cases with short-term postoperative CTAs

TEVAR with adjunct required more than double the fluoroscopy time (77 vs 37 minutes, $p<0.001)$ and significantly longer procedure time $(p=0.005)$, which was highest in the visceral snorkel subgroup (Table 3 ). The adjunct cohort trended toward a higher rate of 
unplanned additional intraoperative maneuvers, three of which were snorkel-related: an inappropriately sized snorkel stent could not be resheathed so was deployed in the external iliac artery, and two celiac snorkel stents dislodged and had to be removed by snare catheter. There were no conversions to open repair.

\section{Early outcomes}

Thirty-day mortality was 5\% (Table 3 ) including one intraoperative death in the TEVAR alone cohort involving a patient with severe tricuspid regurgitation who developed fatal hemorrhage following iliac artery rupture during access sheath advancement. There were two in-hospital deaths, one in the TEVAR alone cohort due to cardiac failure following an episode of hemorrhagic shock and spinal cord ischemia, and one in the adjunct cohort due to embolic mesenteric ischemia resulting in multiorgan system failure.

Major complications are summarized in Table 4. Six (75\%) complications in the TEVAR alone cohort occurred in two patients. In the adjunct cohort, the snorkel stent thrombosis had adequate collateral perfusion via the SMA on diagnostic arteriography precluding the need for further intervention.

Table 4. Major complications

\begin{tabular}{lccc}
\hline & Total $(\mathrm{n}=55)$ & $\begin{array}{c}\text { TEVAR }(\mathrm{n} \\
=29)\end{array}$ & $\begin{array}{c}\text { TEVAR with } \\
\text { adjunct }(\mathrm{n}=26)\end{array}$ \\
\hline Intraoperative hemorrhage with death & 1 & 1 & - \\
\hline Ruptured type IB endoleak requiring stent extension & 1 & - & - \\
\hline New-onset paraplegia & 1 & 1 & - \\
Cerebrovascular accident & 1 & 1 & 1 \\
\hline Distal embolization requiring embolectomy & 2 & 1 & - \\
Hemorrhage requiring repair & 2 & 2 & - \\
\hline Access artery stenosis requiring repair & 1 & 1 & 1 \\
Snorkel occlusion & 1 & - & - \\
\hline New-onset renal failure requiring dialysis & 1 & 1 & \\
\hline
\end{tabular}

A total of 48 cases $(92 \%)$ had short-term postoperative CTAs, all except three within three months of intervention (Table 3 ).

\section{Intermediate-term outcomes}

Excluding the 3 perioperative deaths, median clinical follow-up for the remaining 52 cases was 2.2 years (interquartile range of 0.7 to 3.3 years) with the longest follow-up being 6.5 years. There were 13 deaths (25\%) and 44 cases $(85 \%)$ remained free from reintervention over the follow-up period with no difference between cohorts $(p=0.97$ ) 
(Table 5). Kaplan-Meier analysis (Figure 4) showed no difference between cohorts in combined freedom from reintervention or mortality. The majority of reinterventions $(75 \%)$ involved the distal descending thoracic/thoracoabdominal aorta. There were two open surgical reinterventions including a thoracoabdominal aneurysmectomy with visceral bypass grafting and placement of a four-branch Coselli graft, occurring one and three years after index TEVAR alone respectively.

Figure 4. Stent wedge apposition over time

Short-term CTA (1 month after TEVAR)
Intermediate-term CTA (20 months after TEVAR)

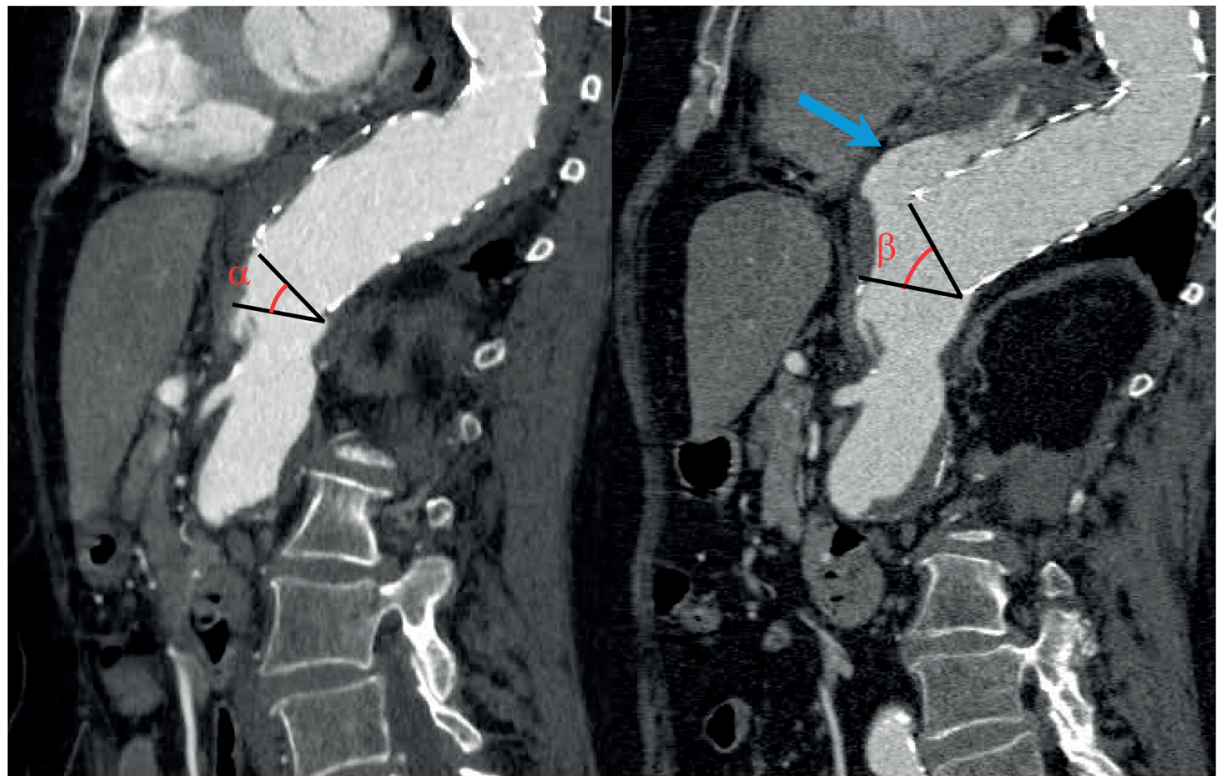

Worsening stent wedge apposition over time (distance $\alpha<\beta$ ) with development of distal stent graft-induced new entry (SINE) and type 1 b endoleak (blue arrow) in an 81-year-old woman after TEVAR alone treatment.

A total of 43 cases (83\%) had intermediate-term postoperative CTAs at a median date of 2.3 years following intervention (interquartile range of 1.0 to 3.2 years). 15 cases (35\%) had type $1 \mathrm{~B}$ endoleaks which occurred at a similar rate between cohorts $(p=0.40)$. There was minimal change in maximal aortic diameter, though 4 cases (9\%) experienced clinically significant maximal aortic diameter growth $(\geq 0.5 \mathrm{~cm} /$ year) with no significant difference between cohorts $(p=0.96)$. Overall the distal LZ grew in diameter however this was not uniform between cohorts: the distal LZ increased by $0.3 \pm 0.6 \mathrm{~cm} /$ year in the TEVAR alone and decreased by $0.1 \pm 0.7 \mathrm{~cm} /$ year in the adjunct cohort $(p=0.04)$. 
Current status of descending thoracic aortic assessment

Table 5. Intermediate-term outcomes

\begin{tabular}{lcccc} 
& Total $(\mathrm{n}=52)$ & $\begin{array}{c}\text { TEVAR } \\
(\mathrm{n}=27)\end{array}$ & $\begin{array}{c}\text { TEVAR with } \\
\text { adjunct }(\mathrm{n}=25)\end{array}$ & p-value \\
\hline Mortality & $13(25)$ & $8(30)$ & $5(20)$ & 0.42 \\
Freedom from reintervention & $44(85)$ & $22(81)$ & $22(88)$ & 0.52 \\
Maximal aortic diameter change $(\mathrm{cm} / \mathrm{yr}) \alpha \beta$ & $0.002 \pm 0.4$ & $-0.09 \pm 0.4$ & $0.1 \pm 0.3$ & 0.25 \\
Maximal aortic growth $\geq 0.5 \mathrm{~cm} / \mathrm{yr}(\mathrm{n}) \alpha \beta$ & $4(9)$ & $2(9)$ & $2(10)$ & 0.96 \\
Distal LZ aortic diameter change $(\mathrm{cm} / \mathrm{yr}) \alpha \beta \chi$ & $0.1 \pm 0.7$ & $0.3 \pm 0.6$ & $-0.1 \pm 0.7$ & 0.04 \\
Distal LZ aortic growth $\geq 0.5 \mathrm{~cm} / \mathrm{yr}(\mathrm{n}) \alpha \beta \chi$ & $8(19)$ & $6(27)$ & $2(21)$ & 0.14 \\
\hline Type 1 B endoleak $\alpha$ & $15(35)$ & $9(41)$ & $6(29)$ & 0.40 \\
Wedge apposition $\geq 1 \mathrm{~cm} \alpha$ & $21(49)$ & $10(45)$ & $11(52)$ & 0.65 \\
LZ stent migration $\geq 1 \mathrm{~cm} \alpha$ & $10(23)$ & $7(32)$ & $3(14)$ & 0.17 \\
\hline
\end{tabular}

$\alpha$ Out of 43 cases with intermediate-term postoperative CTAs $\beta$ Up to 3 outliers were removed

$\chi$ Measured $2 \mathrm{~cm}$ proximal to the rostral celiac ostium

Figure 5. Kaplan-Meier curve of the composite endpoint of freedom from reintervention and mortality

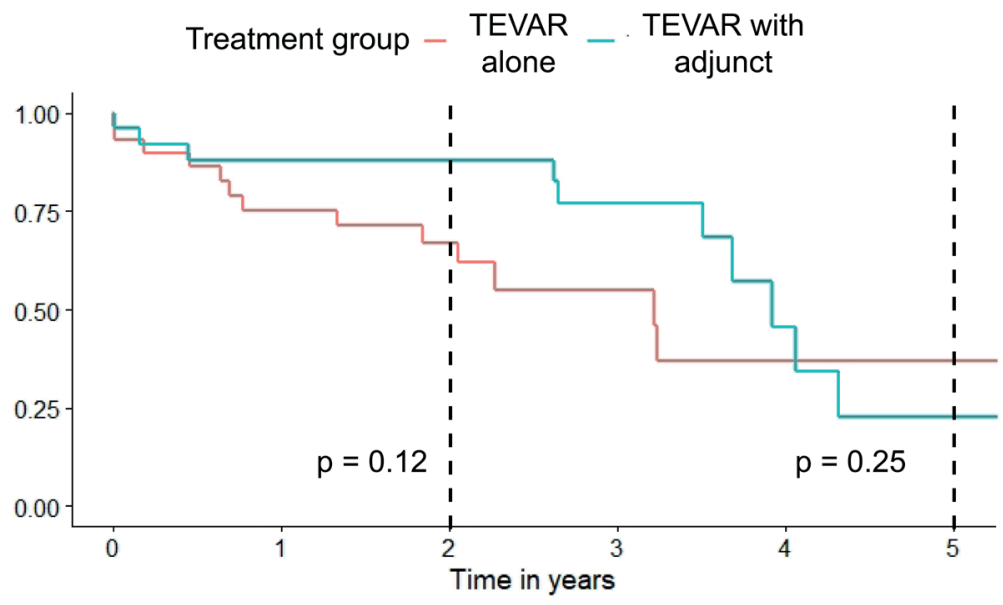

Number at risk

\begin{tabular}{r|cccccc} 
TEVAR alone & 29 & 20 & 14 & 6 & 3 & 3 \\
TEVAR with adjunct & 26 & 18 & 17 & 14 & 4 & 2 \\
\hline & 0 & 1 & 2 & 3 & 4 & 5
\end{tabular}

The impact of treatment approach on primary outcomes was evaluated by multivariate regression analysis, correcting for age and the four CDLZ inclusion parameters. Baseline LZ diameter was found to predict clinically significant maximal aortic diameter growth 
$(p=0.006)$. There was no significant effect on the rate of type $1 B$ endoleaks, freedom from reintervention, or mortality.

\section{COMMENT}

Herein we report our 10-year experience using two distinct endovascular approaches to treat thoracoabdominal aortic aneurysm in the setting of CDLZs: 29 cases received TEVAR alone and 26 TEVAR with adjunct (snorkel with distal stent extension and/or EndoAnchor). Compared to reports on patients with adequate distal LZs, this population has a higher intermediate-term rate of type 1B endoleaks (35\% vs 1-15\%), clinically significant maximal aortic diameter growth (9\% vs $6 \%)$, secondary reintervention $(15 \%$ vs $7 \%$ ), and all-cause mortality (25\% vs $12 \%) . .^{5,8,15-17}$

The comparatively high rate of type $1 \mathrm{~B}$ endoleaks may in part be due to the systematic underreporting of these endoleaks in the literature. ${ }^{7}$ Additionally aortic stent grafts were not post-dilated in LZs with significant mural thrombus precluding a robust distal seal, one that was undermined in other cases by the presence of snorkel gutters. Another likely contributor was the high rate of poor periprocedural stent wedge apposition at $63 \%$, which is known to result in dislodgement and stent migration. ${ }^{18}$

Historically underappreciated, the type $1 \mathrm{~B}$ endoleaks likely contributed to the comparatively high rate of clinically significant maximal aortic diameter growth. The extent of aortic aneurysmal disease in this CDLZ population may also carry a predisposition for further progressive aneurysmal degeneration, a notion supported by the linear regression analysis. The combination resulted in a high secondary reintervention rate. All-cause mortality was expectedly elevated in this non-surgical population.

Comparison of the TEVAR alone and TEVAR with adjunct cohorts, two seemingly distinct patient groups, is warranted by the prevailing similarities in their baseline characteristics and CDLZ anatomy. The differences that did exist between these groups were minor: patients who had failed prior TEVAR were more likely to undergo visceral snorkel with distal stent extension, while those with significant circumferential mural thrombus in the LZ were ineligible for the application of EndoAnchors. Moreover, there was a significant preponderance of adjuncts in the later 2013-2018 time period, suggesting operator experience played a greater role in the selection of treatment modality than the nuances of the CDLZ.

There was no observable difference in primary outcomes between treatment cohorts. TEVAR with adjunct appears to have a protective effect on the distal LZ. This may be due 
to the greater length of perivisceral aorta that can be shielded from constant hemodynamic radial stress with a visceral snorkel and stent extension. Tethering of the distal aorta to a rigid endograft with Aptus Heli-FX EndoAnchors could also provide supporting radial tension, limiting further aneurysmal degeneration. As one of the first reports employing EndoAnchors in the distal LZ, a subanalysis was performed to evaluate the difference in primary outcomes between the snorkel and EndoAnchor subgroups. ${ }^{19}$ Primary outcomes were comparable, however the subgroups were small ( $n=20$ and 6 respectively) and further study is needed. Our experience corroborates the feasibility of placing EndoAnchors in the distal LZ without significant complication.

In conclusion, TEVAR is a viable alternative for the treatment of thoracoabdominal aortic aneurysms in patients with CDLZ who are deemed poor surgical candidates, although with worse intermediate-term outcomes compared to non-CDLZ populations. When comparing currently available TEVAR strategies, the use of adjuncts results in longer fluoroscopy and procedure times with the potential benefit of slowing progressive aneurysmal degeneration of the CDLZ. The combination of these findings argues for the development of branched thoracoabdominal devices to more effectively exclude thoracoabdominal pathology. ${ }^{20,21}$ Until that time, we advocate that patients with CDLZs be carefully evaluated on a case-by-case basis when considering the use of TEVAR alone or with adjuncts 
Thoracic endovascular aortic repair in the setting of compromised distal landing zones

\section{REFERENCES}

1. Riambau V, Böckler D, Brunkwall J, et al. Editor's Choice - Management of Descending Thoracic Aorta Diseases: Clinical Practice Guidelines of the European Society for Vascular Surgery (ESVS). Eur J Vasc Endovasc Surg 2017;53:4-52.

2. Coady MA, Ikonomidis JS, Cheung AT, et al. Surgical management of descending thoracic aortic disease: open and endovascular approaches: a scientific statement from the American Heart Association. Circulation 2010;121:2780-804.

3. Chiu P, Goldstone AB, Schaffer JM, et al. Endovascular Versus Open Repair of Intact Descending Thoracic Aortic Aneurysms. J Am Coll Cardiol 2019;73:643-51.

4. Karimi A, Walker KL, Martin TD, et al. Midterm cost and effectiveness of thoracic endovascular aortic repair versus open repair. Ann Thorac Surg 2012;93:473-9.

5. Svensson LG, Kouchoukos NT, Miller DC, et al. Expert consensus document on the treatment of descending thoracic aortic disease using endovascular stent-grafts. Ann Thorac Surg 2008;85:S1-41.

6. Kölbel T, Panuccio G. More Attention Needed for the Distal Landing Zone in TEVAR. Eur J Vasc Endovasc Surg 2019;58:303-4.

7. Belvroy VM, de Beaufort HWL, van Herwaarden JA, Trimarchi S, Moll FL, Bismuth J. Type 1b Endoleaks After Thoracic Endovascular Aortic Repair are Inadequately Reported: A Systematic Review. Ann Vasc Surg 2019.

8. Berezowski M, Morlock J, Beyersdorf F, et al. Inaccurate aortic stent graft deployment in the distal landing zone: incidence, reasons and consequences. Eur J Cardiothorac Surg 2018;53:1158-64.

9. American Society of Anesthesiologists Classification (ASA Class). 2018. (Accessed January 5, 2020, at http:// www.ncbi.nlm.nih.gov/pubmed/28722969.)

10. Ongstad SB, Miller DF, Panneton JM. The use of EndoAnchors to rescue complicated TEVAR procedures. $J$ Cardiovasc Surg (Torino) 2016;57:716-29.

11. Grabenwöger M, Alfonso F, Bachet J, et al. Thoracic Endovascular Aortic Repair (TEVAR) for the treatment of aortic diseases: a position statement from the European Association for Cardio-Thoracic Surgery (EACTS) and the European Society of Cardiology (ESC), in collaboration with the European Association of Percutaneous Cardiovascular Interventions (EAPCI). Eur J Cardiothorac Surg 2012;42:17-24.

12. Fillinger MF, Greenberg RK, McKinsey JF, Chaikof EL, Standards SfVSAHCoTR. Reporting standards for thoracic endovascular aortic repair (TEVAR). J Vasc Surg 2010;52:1022-33, 33.e15.

13. van Bogerijen GH, Patel HJ, Williams DM, et al. Propensity adjusted analysis of open and endovascular thoracic aortic repair for chronic type B dissection: a twenty-year evaluation. Ann Thorac Surg 2015;99:1260-6.

14. Goudeketting SR, Vermeulen JJM, van Noort K, et al. Effect of Different EndoAnchor Configurations on Aortic Endograft Displacement Resistance: An Experimental Study. J Endovasc Ther 2019;26:704-13.

15. Matsumura JS, Melissano G, Cambria RP, et al. Five-year results of thoracic endovascular aortic repair with the Zenith TX2. J Vasc Surg 2014;60:1-10.

16. Farber MA, Lee WA, Szeto WY, Panneton JM, Kwolek CJ. Initial and midterm results of the Bolton Relay Thoracic Aortic Endovascular Pivotal Trial. J Vasc Surg 2017;65:1556-66.e1.

17. Fairman AS, Beck AW, Malas MB, et al. Reinterventions in the modern era of thoracic endovascular aortic repair. J Vasc Surg 2020;71:408-22.

18. Geisbüsch P, Kotelis D, Hyhlik-Dürr A, Hakimi M, Attigah N, Böckler D. Endografting in the aortic arch - does the proximal landing zone influence outcome? Eur J Vasc Endovasc Surg 2010;39:693-9.

19. Reyes Valdivia A, Busto Suárez S, Duque Santos Á, Zanabili Al-Sibbai AA, Gandarias Zúñiga C, Chaudhuri A. Evaluation of EndoAnchor Aortic Wall Penetration After Thoracic Endovascular Aortic Repair. J Endovasc Ther 2020;27:240-7.

20. Oderich GS, Farber MA, Silveira PG, et al. Technical aspects and 30-day outcomes of the prospective early feasibility study of the GORE EXCLUDER Thoracoabdominal Branched Endoprosthesis (TAMBE) to treat pararenal and extent IV thoracoabdominal aortic aneurysms. J Vasc Surg 2019;70:358-68.e6.

21. Gallitto E, Gargiulo M, Freyrie A, et al. Off-the-shelf multibranched endograft for urgent endovascular repair of thoracoabdominal aortic aneurysms. J Vasc Surg 2017;66:696-704.e5. 


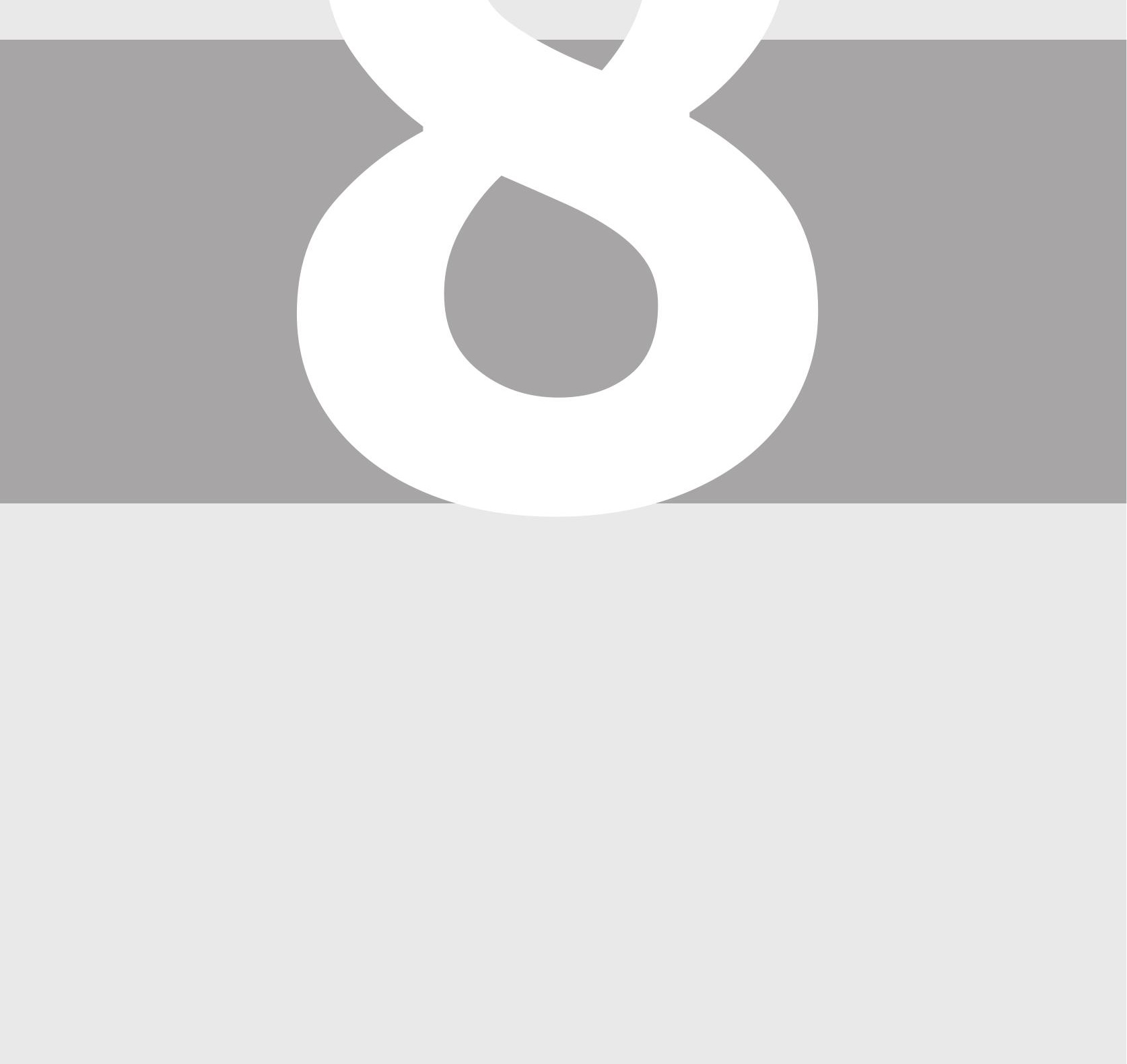




\title{
Mapping pre-dissection aortic wall abnormalities: a multiparametric assessment
}

\author{
Ignas B. Houben, ${ }^{1,2} \mathrm{MD}$ \\ Nitesh Nama, ${ }^{3} \mathrm{PhD}$ \\ Frans L. Moll, ${ }^{2} \mathrm{MD}$, PhD \\ Joost A. van Herwaarden, ${ }^{2} \mathrm{MD}, \mathrm{PhD}$, \\ David A. Nordsletten, ${ }^{1,3} \mathrm{PhD}$, \\ David M. Williams, ${ }^{4} \mathrm{MD}$, \\ Himanshu J. Patel, ${ }^{1} \mathrm{MD}$, \\ C. Alberto Figueroa, ${ }^{3,5} \mathrm{PhD}$, \\ Nicholas S. Burris, ${ }^{4}$ MD.
}

${ }^{1}$ Department of Cardiac Surgery, Frankel Cardiovascular Center, University of Michigan, Ann Arbor, United States
${ }^{2}$ Department of Vascular Surgery, Utrecht University, Utrecht, The Netherlands
${ }^{3}$ Department of Biomedical Engineering, University of Michigan, Ann Arbor, United States
${ }^{4}$ Department of Radiology, University of Michigan, Ann Arbor, United States
${ }^{5}$ Department of Vascular Surgery, Frankel Cardiovascular Center, University of Michigan, Ann Arbor, United States

Eur J Cardiothorac Surg. 2020;57(6):1061-1067.

doi: 10.1093/ejcts/ezz381. 


\section{ABSTRACT}

\section{Objective}

Maximal aortic diameter is commonly used to assess aortic risk, but poorly predicts the timing and location of dissection events in patients with connective tissue disease (CTD) who underwent regular imaging surveillance. Hence, we aimed to use available surveillance computed tomography angiography (CTA) scans to investigate the correlation between three-dimensional (3D) growth and cyclic transmural wall stress with the location of intimal tear formation.

\section{Methods}

Three type B aortic dissection (TBAD) patients with two available ECG-gated predissection CTA scans and without surgical repair during the pre-dissection interval were retrospectively identified at our institution. Vascular Deformation Mapping (VDM) was used to measure 3D aortic growth between two pre-dissection clinical CTA studies. Additionally, we performed a computational analysis to estimate cyclic transmural wall stress in patient-specific baseline CTA geometries.

\section{Results}

In all three CTD patients, the site of type B aortic intimal tear co-localized with areas of peak 3D aortic wall growth. Aortic growth was detected by clinical radiologic assessment in only one case. Co-localization of peak transmural stress and the site of intimal tear formation was found in all cases.

\section{Conclusion}

Focal areas of growth and transmural wall stress co-localized with the site of intimal tear formation. These hypothesis-generating results suggest a possible new analytic pathway for a more sophisticated assessment of the factors leading to initiation of dissection in CTD patients. These methods could improve on current risk-stratification techniques.

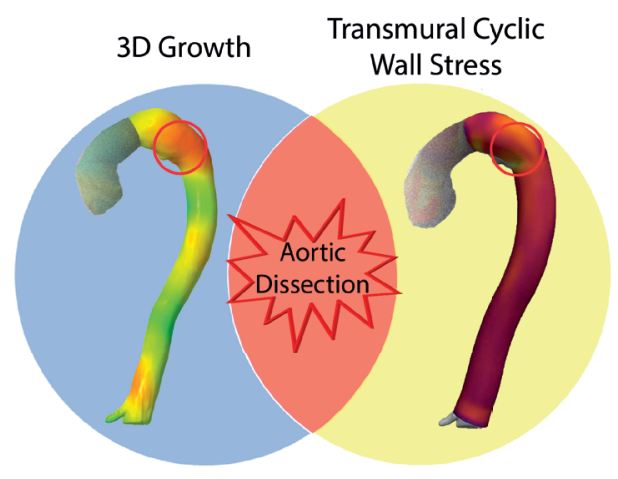




\section{INTRODUCTION}

Type $B$ aortic dissection (TBAD) is a life-threatening condition that requires acute medical attention with antihypertensive treatment and potential surgical intervention. ${ }^{1,2}$ Despite significant advances in surgical techniques for the treatment of TBAD, the ability to predict the timing and location of dissection formation is lacking. A variety of clinical risk factors for TBAD are known, such as advanced age, hypertension, atherosclerosis and connective tissue disease (CTD). ${ }^{3,4}$ Specifically, patients with CTD (e.g., Marfan syndrome) have a high lifetime risk of dissection and undergo regular imaging surveillance both in the pre-surgical setting to monitor aortic aneurysm and other complications, but also in the post-surgical setting to monitor for surgical complications and continued growth or dissection of the residual native aorta. Currently, the primary metric used to estimate the risk of dissection and inform candidacy for surgical management is maximal aortic diameter. ${ }^{5,6}$ Unfortunately, this metric is a poor predictor of dissection development, with a recent study estimating that approximately $80 \%$ of dissection patients (both type $A$ and type $B$ ) have maximal aortic diameter below standard surgical thresholds $\left(5.5 \mathrm{~cm}\right.$ ) at the time of dissection. ${ }^{7,8}$ In a study of Marfan patients who developed type $B$ dissection during the imaging surveillance, the average pre-dissection diameter of the affected segment was normal at $28 \mathrm{~mm}$, highlighting a critical need for better risk assessment techniques in this population. ${ }^{9}$

Aortic growth is a complex phenomenon driven by a wide range of biomechanical processes such as inflammation, intrinsic structural defects related to CTD, wall constituent turnover and hypertension. ${ }^{10}$ While the exact sequence of events leading to the initiation of aortic dissection remains poorly understood, pathologic, computational and animal studies have demonstrated common pathways of medial degeneration, including mucoid extracellular matrix accumulation, elastic fiber fragmentation and smooth muscle cell necrosis, that result in progressive loss of aortic wall integrity and ultimately the intimal tearing and wall delamination. ${ }^{11}$ Recent reports have highlighted that the local transmural wall stress during a cardiac cycle can influence long-term growth and remodeling of the aortic wall through stress-mediated changes in aortic wall tissue composition. ${ }^{12-14}$ Additionally, a prior study investigating the biomechanical effects of pooling of proteoglycans within the aortic wall - a common abnormality in CTD - demonstrated that such accumulations and the resultant osmotic swelling can lead to localized concentrations of wall stress that are sufficient to disrupt the extracellular matrix and initiate tearing. ${ }^{15}$

Regardless of the exact mechanisms of aortic dissection initiation, the progressive loss of aortic wall integrity caused by medial degeneration also results in aortic growth, a concept that has been termed as mechanobiological instability. ${ }^{16}$ However, aortic 
growth is often slow and small in magnitude, and thus undetected by current diameterbased measurement approaches due to the high degree of measurement variability (on the order of $\pm 1-5 \mathrm{~mm}) .{ }^{17}$ Vascular deformation mapping (VDM) is a recently developed image analysis technique which may overcome these limitations. ${ }^{18}$ VDM measures three-dimensional (3D) aortic wall deformation between two computed tomography angiography (CTA) scans. This 3D deformation allows for an accurate characterization of the localized patterns of aortic wall enlargement that could help detect early adverse aortic remodeling leading to acute adverse events.

In this study, we investigate the hypothesis that areas of focal aortic enlargement, via their association with wall instability, will co-localize spatially with the site of intimal tear in patients with thoracic aortic disease and CTD who developed aortic dissection during the course of clinical imaging surveillance. Furthermore, we investigate the relationship between the location of intimal tear formation, computationally obtained spatial distribution of cyclic aortic wall stress, and the pattern of long-term aortic wall enlargement obtained via VDM analysis.

\section{METHODS}

\section{Patient identification and characteristics}

Subjects were identified retrospectively from an internal database of patients referred to the Departments of Cardiac Surgery and Interventional Radiology at our institution with available pre-dissection CTA imaging between January 2011 and January 2019. These patients were recruited to an Institutional Review Board approved study, (Protocol \#HUM00133798), approved by 08/18/2017 with waiver of informed consent. During this period, we identified a total of 31 patients with pre-dissection CTA scans, although 12 were excluded for having only one pre-dissection CTA (the VDM growth analysis requires two studies), 8 patients were excluded due to non-ECG-gated CTA scans, 5 patients were excluded for surgical repair of an adjacent segment in the pre-dissection intervals, and finally 2 patients were excluded for poor quality CT images (e.g., poor contrast opacification, motion artifact). Of the remaining four patients, three had confirmed diagnosis of CTD, and thus in order to maximize homogeneity of the population, the single nonconnective tissue disease patient was excluded.

\section{CTA imaging}

All CTA studies were performed at our institution on a 64-slice scanner (GE Healthcare, Milwaukee, WI, USA) after intravenous injection of $120 \mathrm{~mL}$ iopamidol contrast (Isoview 370, Bracco Diagnostics, Milan, Italy). ECG-gating was used in all CTA studies. CTA data 
was reconstructed at $75 \%$ of the R-R interval with $1.25 \mathrm{~mm}$ slice thickness and $0.625 \mathrm{~mm}$ slice interval.

\section{Vascular deformation mapping}

Methods for vascular deformation mapping have been described in detail in previous work. ${ }^{12}$ However, in brief, the VDM technique involves the following steps:

1) Segmentation - The thoracic aorta, from the aortic valve to $10 \mathrm{~mm}$ beyond the celiac artery, is first segmented using a threshold-based approach followed by manual adjustments and the surface structure is based on this segmentation. Aortic segmentation and automated centerline calculation is performed using Mimics 21.0 (Materialise NV, Leuven, Belgium) and the segmentation is also used to generate a mask which is used to constrain the registration step.

2) Image registration - Deformable image registration was performed between serial CTA studies using a custom Matlab interface to the Elastix open source software (Utrecht, Netherlands). Registration was performed in two steps, first with an affine registration to approximately align the two segmentations, followed by non-rigid, multi-resolution $B$-spline registration using mutual information as the similarly metric.

3) Deformation quantification - Deformation between pre-dissection CTA studies was quantified as the determinant of the 3D spatial Jacobian calculated from the final optimized image transform and normalized by the time interval (|J|/year) to obtain a deformation rate. The Jacobian determinant represents volumetric change, and thus a growth of $0.2 \mathrm{~J} / \mathrm{yr}$ at a point on the aortic wall surface can be interpreted as a $20 \%$ volumetric change over the time interval at that point. Deformation rate values ( $\mathrm{J} / \mathrm{year}$ ) were linearly interpolated to the vertex points of the baseline aortic segmentation surface for display. Positive values of J represent aortic wall expansion (yellow-red color scale), aortic wall compression by $\mathrm{J}$ values $<0$ (blue), and unchanged aortic wall geometry by $\mathrm{J} \approx 0$ (green).

\section{Computational stress analysis}

An aortic model was constructed from the baseline mid-diastolic phased CTA image data using the in-house computational hemodynamics modelling environment, CRIMSON (CardiovasculaR Integrated Modeling and SimulatiON, www.crimson.software). ${ }^{19}$ The vessel wall geometry was obtained by creating $2 \mathrm{D}$ segmentation contours along the vessel centerline, followed by subsequent lofting to create a geometric CAD model. The aortic wall was modelled as a thin shell using a finite element formulation. To account for the nonlinear behavior of vessel wall, the aortic wall is characterized by a Neo- 
Hookean material model. The Neo-Hookean model material stiffness constant (c) and wall thickness $(\mathrm{t})$ were varied from $\mathrm{c}=1 \mathrm{MPa}, \mathrm{t}=2 \mathrm{~mm}$ for the ascending aorta to $\mathrm{c}=2 \mathrm{MPa}$, $\mathrm{t}=1.6 \mathrm{~mm}$ for the descending aorta. ${ }^{15,20}$ For the Dacron graft, uniform coefficients were assigned to the entire surface: $\mathrm{c}=50 \mathrm{MPa}$ and $\mathrm{t}=0.5 \mathrm{~mm} \cdot{ }^{15,20}$ For each subject, the aortic model was first prestressed to the patient-specific diastolic pressure (listed in Table 1) using a forward incremental prestress strategy. ${ }^{21}$ This procedure identifies the in-vivo diastolic state of stress in the aortic wall. Subsequently, the aortic wall is loaded from diastolic pressure to systolic pressure to identify the distribution of cyclic transmural wall stresses. A simplified conceptual workflow of both the VDM and the computational analysis approaches of this work is displayed in Figure 1.

Table 1 Patient characteristics including antihypertensive medication and the last reported blood pressure medication before the onset of dissection.

\begin{tabular}{|c|c|c|c|c|c|c|c|}
\hline Patient & Age & Gender & Comorbidities & Prior surgery & $\begin{array}{l}\text { Antihypertensive } \\
\text { medication }\end{array}$ & $\begin{array}{c}\text { BP at } \\
\text { baseline } \\
\text { CTA } \\
\text { (mmHg) }\end{array}$ & $\begin{array}{c}\text { BP at } \\
\text { follow- } \\
\text { up CTA } \\
\text { (mmHg) }\end{array}$ \\
\hline 1 & 30 & Female & $\begin{array}{c}\text { Marfan } \\
\text { Syndrome }\end{array}$ & $\begin{array}{l}\text { Bentall procedure, } \\
\text { Mitral valve repair }\end{array}$ & $\begin{array}{c}\text { Furosemide } 25 \mathrm{mg} \\
\text { daily, Atenolol } \\
25 \mathrm{mg} \text { daily }\end{array}$ & $109 / 73$ & $124 / 72$ \\
\hline 2 & 67 & Male & $\begin{array}{l}\text { Hypertension, } \\
\text { Dyslipidaemia }\end{array}$ & $\begin{array}{l}\text { Ascending tube } \\
\text { graft with TAR }\end{array}$ & $\begin{array}{l}\text { Metoprolol } 50 \mathrm{mg} \\
\text { daily }\end{array}$ & $140 / 65$ & $118 / 61$ \\
\hline 3 & 52 & Male & $\begin{array}{c}\text { Marfan } \\
\text { syndrome, } \\
\text { Hypertension, } \\
\text { Dyslipidaemia }\end{array}$ & David procedure & $\begin{array}{l}\text { Furosemide } 20 \mathrm{mg} \\
\text { daily, } \\
\text { Metoprolol } 50 \mathrm{mg} \\
\text { daily }\end{array}$ & $104 / 65$ & $120 / 80$ \\
\hline 4 & 40 & Male & $\begin{array}{l}\text { Loeys-Dietz } \\
\text { syndrome, } \\
\text { Hypertension, } \\
\text { Dyslipidaemia }\end{array}$ & $\begin{array}{l}\text { AVR with ascending } \\
\text { tube repair, Re-do } \\
\text { ascending repair } \\
\text { with TAR }\end{array}$ & $\begin{array}{l}\text { Metoprolol } 100 \mathrm{mg} \\
\text { daily }\end{array}$ & $114 / 65$ & $145 / 80$ \\
\hline
\end{tabular}

AVR = aortic valve replacement; TAR = total arch replacement; $B P=$ blood pressure; $C T A=$ computed tomography angiography

Figure 1 Overview of methodology of A) 3-dimensional growth analysis through a vascular deformation map (VDM) and B) computational analysis of transmural wall stress.

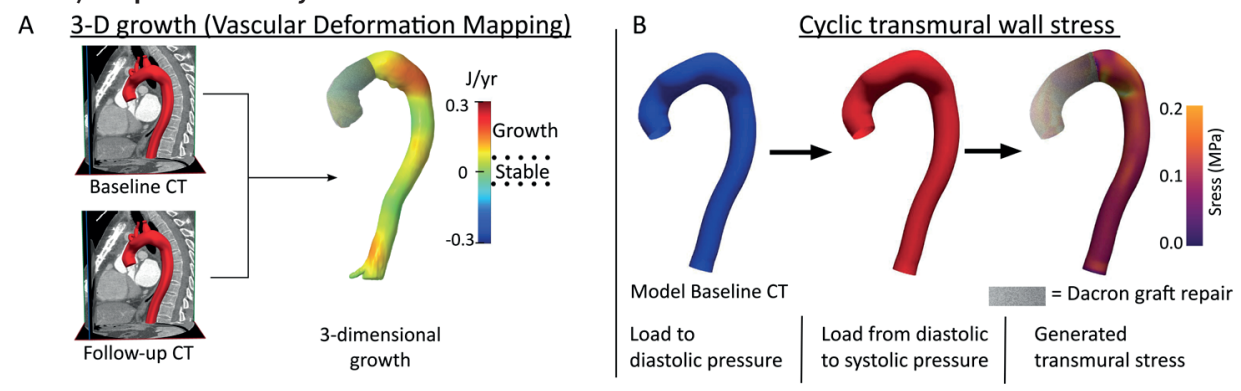




\section{RESULTS}

Three patients are described in this series and will be discussed below on a case-by-case manner. A summary of the patient demographics and timeline is provided in Table 1 . Antihypertensive medication, dose and frequency for each patient were constant over the entire course of surveillance imaging leading up to the dissection. Table 2 summarizes the 3-D growth, transmural wall stress and CTA follow-up for each patient.

Table 2 Summary of 3-D growth and transmural wall stress outcomes for each patient

\begin{tabular}{lcccc}
\hline & Case 1 & Case 2 & Case 3 & Case 4 \\
\hline Follow-up baseline to second CTA (years) & 2.3 & 3.1 & 2.1 & 2.1 \\
2-D diameter change (mm/yr) & 1.8 & 0.9 & 1.0 & 1.5 \\
Peak growth (J/yr) & 0.29 & 0.08 & 0.14 & 0.25 \\
Peak stress (MPa) & 0.35 & 0.16 & 0.14 & 0.14 \\
Follow-up second CTA to dissection (months) & 11.7 & 18.4 & 3.5 & 11.8 \\
\hline
\end{tabular}

CTA = computed tomography angiography

\section{Patient 1}

The first patient was a 30-year-old female with Marfan syndrome and scoliosis, a prior history of Bentall repair at age 15 and multiple prior mitral valve procedures. She was an active smoker at baseline CT-scan, but had no other risk-factors for cardiovascular disease. The interval between her two surveillance CT scans was 2.3 years and her thoracoabdominal aorta was reported to have enlarged from 3.6 to $4.2 \mathrm{~cm}$ in maximal diameter over that period by clinical radiologic assessment. The recorded pulse pressure at the baseline CT scan and the follow-up CT scan increased from 36 to $52 \mathrm{mmHg}$. By VDM analysis, peak growth was found in the supraceliac aorta region (0.29 J/year) and co-localized with the site of subsequent intimal tear formation. The peak stress value $(0.35 \mathrm{MPa}$ ) was observed in this same region, approximately $1 \mathrm{~cm}$ proximal to the origin of the celiac trunk (Figure 2). The time interval between the second CT-scan and the date of dissection was 12 months.

\section{Patient 2}

The second patient was a 52-year-old male with Marfan syndrome, hypertension, dyslipidemia and a history of ascending aortic aneurysm, who underwent valve-sparing repair 20 months prior to baseline surveillance scan. His blood pressure had been well-controlled with oral metoprolol $25 \mathrm{mg}$ twice daily and furosemide $20 \mathrm{mg}$ daily. Pulse pressure was $39 \mathrm{mmHg}$ at baseline CT-scan and $40 \mathrm{mmHg}$ at the follow-up CT scan. The interval between his two surveillance CT-scans was 2.1 years. Radiology reports did not describe any significant changes between the two surveillance CTA studies. A focal area of growth $(0.14 \mathrm{~J} / \mathrm{yr})$ was identified by VDM along the posterior wall of the distal 


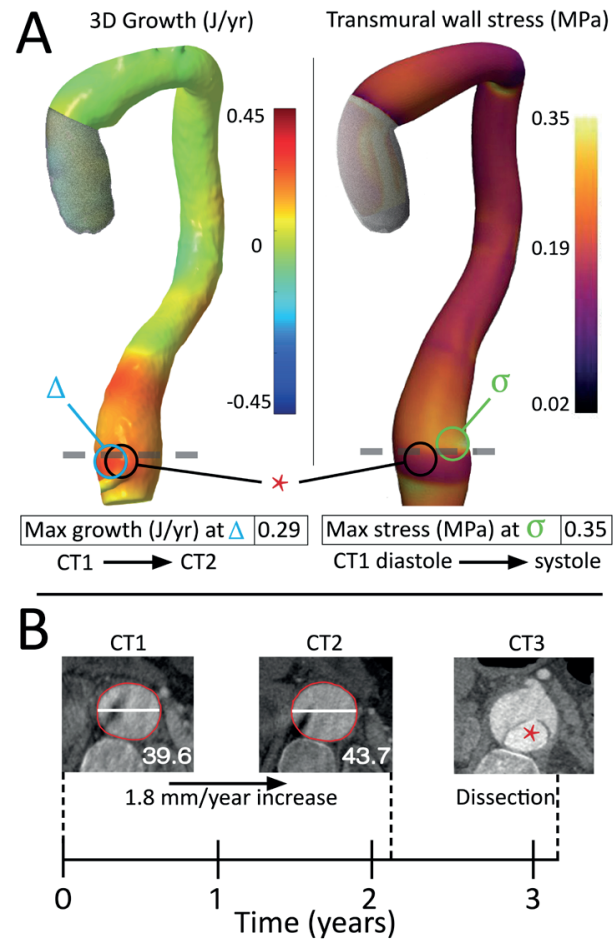

\section{Figure 2: Results patient 1}

Three-dimensional growth ( $\mathrm{A}$, left) and computational cyclic transmural stress (A, right) results for patient 1 . The site of intimal tear (star) co-localized with the region of peak growth (delta) and the region of peak transmural stress (sigma) in the supraceliac aorta. Serial CT images at the level of subsequent tear are shown below (B). The greyed area reflects graft material from aortic repair. Of note, intraluminal artifact from metallic spinal hardware is seen on CT1 and CT2 images.

arch in close proximity of the location of the subsequent intimal tear. The site of peak transmural wall stress $(0.14 \mathrm{MPa})$ was located along the posterior distal arch at the lesser curvature, in close proximity and just proximal to the site of the intimal tear (Figure 3 ). The time interval between the follow-up scan and the date of dissection was 3.5 months.

\section{Patient 3}

The third patient was a 40-year-old male with Loeys-Dietz syndrome, hypertension, dyslipidemia and a history of DeBakey type II aortic dissection, which had been previously repaired at an outside hospital. However, a redo repair was performed at our institution due to residual dissection and proximal growth, using a modified root repair due to frail sinus and coronary tissue, with concurrent ascending aortic and total arch repairs, including the innominate and left carotid artery. During the post-operative period, blood pressure remained well-controlled over a 2.1 year interval between surveillance CT scans using oral metoprolol $100 \mathrm{mg}$ daily with a pulse pressure of $49 \mathrm{mmHg}$ at the baseline scan and $65 \mathrm{mmHg}$ at the follow-up scan. Peak growth was $0.25 \mathrm{~J} / \mathrm{yr}$ and was measured at the distal aortic arch. Peak transmural wall stress was $0.14 \mathrm{MPa}$, located at the distal aortic arch. The sites of peak growth and peak transmural stress co-localized with the region of subsequent intimal tear (Figure 4). 
Mapping pre-dissection aortic wall abnormalities: a multiparametric assessment
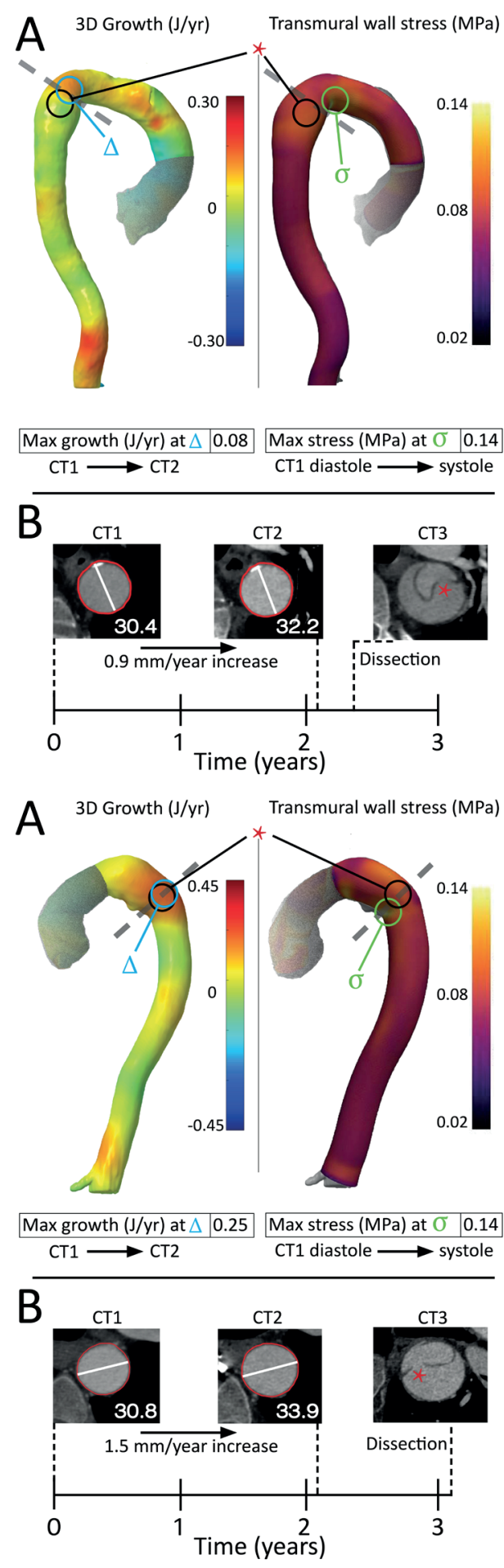

\section{Figure 3: Results Patient 2}

Three-dimensional growth (A, left) and computational cyclic transmural stress (A, right) results for patient 2 . The site of intimal tear (star) co-localized with a region of peak growth (delta) and the region of peak transmural stress (sigma) in the distal arch. Serial CT images at the level of subsequent tear are shown below (B). The greyed area reflects graft material from aortic repair.

\section{Figure 4: Results Patient 3}

Three-dimensional growth ( $A$, left) and computational cyclic transmural stress (A, right) results for patient 3 . The site of intimal tear (star) co-localized with a region of peak growth (delta) and the region of peak transmural stress (sigma) in the distal arch. Serial CT images at the level of subsequent tear are shown below (B). The greyed area reflects graft material from aortic repair. 


\section{DISCUSSION}

Despite the poor performance of aortic diameter in predicting the risk of dissection, with $50-60 \%$ of patients dissecting at a normal or mildly dilated aortic diameter $(<45$ $\mathrm{mm}),{ }^{7,8}$ it remains the most commonly used metric in clinical practice. Key limitations of a diameter-based approach are that this method represents aortic growth as a circumferentially uniform process, measures growth primarily at the site of maximal aortic diameter, and is characterized by a high degree of measurement variability. Furthermore, diameter measurements overlook the three-dimensional features of aortic growth. A final potential reason for the obvious disconnect between diameter and dissection risk may be that three-dimensional growth is a dynamic measure, unlike maximal diameter, and may be more reflective of the underlying pathologic factors that eventually lead to long-term growth and progressive loss of wall integrity. It seems logical that an actively growing $4 \mathrm{~cm}$ diameter aorta has less structural integrity than a stable $6 \mathrm{~cm}$ aorta.

Beyond morphologic changes such as aortic growth, there have been recent reports indicating the role of transmural wall stress driving long-term growth and remodeling of aortic tissue. ${ }^{22,23}$ For instance, in the context of hypertension, increased blood pressure leads to locally elevated circumferential wall stress which promotes matrix synthesis, often via local production of angiotensin-II, and exacerbates arterial wall stiffening. This stiffening further causes an increase in the pulse wave velocity that can lead to augmented central blood pressure, thereby initiating an insidious positive feedback loop that results in progressive worsening. ${ }^{14}$ Additionally, prior computational research has suggested that the accumulation of pools of proteoglycans within the wall and subsequent osmotic swelling can lead to localized concentrations of wall stress that may initiate intimal tearing, ${ }^{15}$ and recent evidence has confirmed such accumulations through clinical pathologic samples of CTD patients with aortic dissection. ${ }^{24}$ Despite these reports, the contribution of cyclical transmural wall stress in driving long-term adverse aortic events such as aortic dissection has not been explored in detail.

To address these issues, we combine a three-dimensional assessment of aortic growth using an advanced image analysis technique, Vascular Deformation Mapping, and a computational analysis of cyclic transmural wall stress. We submit that the VDM may be better-suited to investigate the link between aortic dimensions and the risk of aortic dissection given that it is less prone to measurement variability and capable of depicting of the 3D nature of growth. Furthermore, a computational analysis of cyclic transmural stress in baseline CTA geometry could indicate the areas of aortic wall that are most vulnerable to aortic dissection due the reported stress-mediated turnover of aortic constituents reported in earlier growth and remodeling reports. ${ }^{12-14}$ This two-pronged 
approach can allow for a sophisticated morphologic and biomechanical assessment that may improve our current understanding of the pathogenesis of aortic dissection.

Our results revealed that the site of intimal tear co-localized with both focal areas of aortic growth and with cyclic transmural wall stress, supporting our hypothesis that the risk of dissection may be highest at the locations where insufficient wall integrity (e.g., growth) and abnormal wall stress intersect. Our study consisted of three patients who all suffered from connective tissue disease. Due to an imbalance in the signaling pathway of transforming growth factor beta (TGF- $\beta$ ) and associated downstream cellular and extracellular structural alterations, patients with CTD are known to exhibit an accelerated growth profile compared to patients without CTD. ${ }^{25}$ It has been suggested that prior aortic repair is an increased risk factor for type $B$ aortic dissection in patients with Marfan syndrome, as was observed in all three patients in our study. ${ }^{9}$ The preliminary findings in this study would be more optimally validated if the outcomes of our current series where to be compared to both CTD patients with dissection but without any prior aortic repair, however, serial CTA studies in this population were not available for analysis at our center.

To our knowledge, the results of this study are the first to demonstrate spatial correlation between the site of intimal tear dissection, peak cyclic transmural wall stress and peak focal growth using clinical imaging data from TBAD patients. Our results, while admittedly still preliminary, suggest a potential new approach to determine risk of dissection in CTD patients undergoing imaging surveillance for thoracic aortic disease. Non-invasive assessment of focal growth (rather than the maximal diameter) and cyclical transmural wall stress may more closely represent pathologic changes of the aortic wall that are currently thought to play a role in the initiation of aortic dissection but which are difficult to assess using currently available methods. Considering that both the VDM and the computational technique utilize routine ECG-gated CTA data - the mainstay of aortic disease surveillance - if future research confirms the predictive value of the proposed computational indices presented here, these findings would be poised for translation into clinical practice.

\section{Limitations}

There are several limitations to this study, which include the following:

1) The number of patients in this study was small, as we were limited to patients with 2 available pre-dissection ECG-gated CTAs to perform VDM analysis, which is an uncommon occurrence. additionally, all three patients had CTD, so while this may only represent a small fraction of patients with TBAD, these patients are at higher 
risk of dissection and are more likely to receive regular imaging surveillance, so our findings may be best applied to this population.

2) There was a variable amount of time between last surveillance CT and dissection event (between about 4-12 months), however, we were limited by available CT data, and while the exact changes in aortic wall geometry closer to the date of dissection are unknown, it is reassuring that areas of aortic growth co-localized with the site of intimal tear in all cases.

3) We were not able to assess the potential co-localization of abnormal hemodynamics (e.g., elevated wall shear stress) with site of intimal tear, mostly owing to a lack of patient-specific hemodynamic data (echo or phase-contrast MRI). However, the contribution of abnormal WSS in dissection of the ascending aorta (type A) related to post-stenotic jets, and less so in dissection of the descending thoracic aorta (type B) as in all of the patients in our study. ${ }^{26}$

4) This work utilized a nonlinear thin shell description for the aortic wall via a NeoHookean material model using available literature data for tissue properties. Alternative multi-layer material models and use of patient-specific material parameters can further improve the computational predictions of aortic wall stress. Future studies will aim to obtain blood pressures, next to multiphase ECG-gated CTA data, in order to derive the patient-specific structural stiffness through distensibility assessment.

\section{CONCLUSION}

In conclusion, this multi-parametric analysis co-localization of pre-dissection focal aortic growth and cyclic transmural stress with the site of intimal tear in TBAD patients. Our results support the roles of stress concentrations and loss of aortic wall structural integrity that have been suggested to contribute to dissection initiation. While future work is needed to validate our findings in a larger population and compare to a cohort of patients who did not develop dissection events during imaging surveillance, we believe the results of this study support our hypothesis on the mechanisms driving aortic growth and should motivate further investigation into the prognostic significance of transmural wall stress and VDM growth mapping, and most importantly their intersection. Given the recent availability of such advanced aortic wall analysis techniques, we may finally be approaching a point where dissection risk-stratification can move from a poorly predictive diameter-based approach to a sophisticated morphologic and biomechanical assessment that better aligns with our current understanding of the pathogenesis of aortic dissection initiation. 
Mapping pre-dissection aortic wall abnormalities: a multiparametric assessment

\section{REFERENCES}

1. Hiratzka LF, Bakris GL, Beckman JA, Bersin RM, Carr VF, Casey DE, et al. 2010 ACCF/AHA/AATS/ACR/ASA/SCA/ SCAI/SIR/STS/SVM Guidelines for the Diagnosis and Management of Patients with Thoracic Aortic Disease. Anesth Analg. 2010;111(2):279-315. doi:10.1016/j.jacc.2010.02.010

2. Riambau V, Böckler D, Brunkwall J, Cao P, Chiesa R, Coppi G, et al. Management of Descending Thoracic Aorta Diseases: Clinical Practice Guidelines of the European Society for Vascular Surgery (ESVS). Eur J Vasc EndovasC Surg. 2017;53(1):4-52. doi:10.1016/j.ejvs.2016.06.005

3. Collins JS, Evangelista A, Nienaber CA, Bossone E, Fang J, Cooper JV, et al. Differences in clinical presentation, management, and outcomes of acute type A aortic dissection in patients with and without previous cardiac surgery. Circulation. 2004;110(11 SUPPL.). doi:10.1161/01.CIR.0000138219.67028.2a

4. Vilacosta I, Aragoncillo P, Cañadas V, San Román JA, Ferreirós J, Rodríguez E. Acute aortic syndrome: A new look at an old conundrum. Heart. 2009;95:1130-1139. doi:10.1136/hrt.2008.153650

5. Elefteriades JA, Farkas EA. Thoracic Aortic Aneurysm. Clinically Pertinent Controversies and Uncertainties. J Am Coll Cardiol. 2010;55(9):841-857. doi:10.1016/j.jacc.2009.08.084

6. Kim JB, Spotnitz M, Lindsay ME, MacGillivray TE, Isselbacher EM, Sundt TM. Risk of Aortic Dissection in the Moderately Dilated Ascending Aorta. J Am Coll Cardiol. 2016;68(11):1209-1219. doi:10.1016/j.jacc.2016.06.025

7. Trimarchi S, Jonker FHW, Froehlich JB, Upchurch GR, Moll FL, Muhs BE, et al. Acute type B aortic dissection in the absence of aortic dilatation. J Vasc Surg. 2012;56(2):311-316. doi:10.1016/j.jvs.2012.01.055

8. Berezowski M, Kosiorowska K, Beyersdorf F, Riesterer T, Jasinski M, Plonek T, et al. Modelling of predissection aortic size in acute descending aortic dissection. Interact Cardiovasc Thorac Surg. 2019;29(1):124-129. doi:10.1093/icvts/ivz028

9. Den Hartog AW, Franken R, Zwinderman AH, Timmermans J, Scholte AJ, van den Berg MP, et al. The risk for type B aortic dissection in Marfan syndrome. J Am Coll Cardiol. 2015;65(3):246-254. doi:10.1016/j.jacc.2014.10.050

10. Goldfinger JZ, Halperin JL, Marin ML, Stewart AS, Eagle KA, Fuster V. Thoracic aortic aneurysm and dissection. J Am Coll Cardiol. 2014;64(16):1725-1739. doi:10.1016/j.jacc.2014.08.025

11. Halushka MK, Angelini A, Bartoloni G, Basso C, Batoroeva L, Bruneval P, et al. Consensus statement on surgical pathology of the aorta from the Society for Cardiovascular Pathology and the Association For European Cardiovascular Pathology: II. Noninflammatory degenerative diseases - nomenclature and diagnostic criteria. Cardiovasc Pathol. 2016;25(3):247-257. doi:10.1016/j.carpath.2016.03.002

12. Valentin A, Humphrey JD, Holzapfel GA. A finite element-based constrained mixture implementation for arterial growth, remodeling, and adaptation: Theory and numerical verification. Int J Numer Meth Biomed Engng. 2013;29:822-849. doi:10.1002/cnm.2555

13. Bersi MR, Bellini C, Wu J, Montaniel KRC, Harrison DG, Humphrey JD. Excessive adventitial remodeling leads to early aortic maladaptation in angiotensin-induced hypertension. Hypertension. 2016;67(5):890-896. doi:10.1161/HYPERTENSIONAHA.115.06262

14. Humphrey JD, Harrison DG, Figueroa CA, Lacolley P, Laurent S. Central Artery stiffness in hypertension and aging a problem with cause and consequence. Circ Res. 2016;118(3):379-381. doi:10.1161/CIRCRESAHA.115.307722

15. Roccabianca S, Bellini C, Humphrey JD. Computational modelling suggests good, bad and ugly roles of glycosaminoglycans in arterial wall mechanics and mechanobiology. J R Soc Interface. 2014;11(97). doi:10.1098/ rsif.2014.0397

16. Cyron CJ, Wilson JS, Humphrey JD. Mechanobiological stability: A new paradigm to understand the enlargement of aneurysms? J R Soc Interface. 2014;11(100). doi:10.1098/rsif.2014.0680

17. Asch FM, Yuriditsky E, Prakash SK, Siddharth K, Roman MJ, Weinsaft JW, et al. The Need for Standardized Methods for Measuring the Aorta Multimodality Core Lab Experience from the GenTAC Registry. JACC CardiovasC Imaging. 2016;9(3):219-226. doi:10.1016/j.jcmg.2015.06.023

18. Burris NS, Hoff BA, Kazerooni EA, Ross BD. Vascular Deformation Mapping (VDM) of Thoracic Aortic Enlargement in Aneurysmal Disease and Dissection. Tomography. 2017;3(3):163-173. doi:10.18383/j.tom.2017.00015

19. Arthurs CJ, Figueroa CA. CRIMSON Software, Documentation and Downloads. http://www.crimson.software/ downloads.html. Published 2019. Accessed January 10, 2019. 
Current status of descending thoracic aortic assessment

20. Tremblay D, Zigras T, Cartier R, Leduc L, Butany J, Mongrain R, et al. A Comparison of Mechanical Properties of Materials Used in Aortic Arch Reconstruction. Ann Thorac Surg. 2009;88(5):1484-1491. doi:10.1016/j.athoracsur.2009.07.023

21. Grytz R, Downs JC. A forward incremental prestressing method with application to inverse parameter estimations and eye-specific simulations of posterior scleral shells. Comput Methods Biomech Biomed Engin. 2013;16(7):768-780. doi:10.1080/10255842.2011.641119

22. Taber LA, Humphrey JD. Stress-modulated growth, residual stress, and vascular heterogeneity. J Biomech Eng. 2001;123(6):528-535. doi:10.1115/1.1412451

23. Holzapfel GA, Gasser TC, Ogden RW. A new constitutive framework for arterial wall mechanics and a comparative study of material models. J Elast. 2000;61(1-3):1-48. doi:10.1023/A:1010835316564

24. Cikach FS, Koch CD, Mead TJ, Galatioto J, Willard BB, Emerton KB, et al. Massive aggrecan and versican accumulation in thoracic aortic aneurysm and dissection Find the latest version : Massive aggrecan and versican accumulation in thoracic aortic aneurysm and dissection. JCl Insight. 2018;3(5).

25. Humphrey JD. Possible mechanical roles of glycosaminoglycans in thoracic aortic dissection and associations with dysregulated transforming growth factor- $\beta$. J Vasc Res. 2012;50(1):1-10. doi:10.1159/000342436

26. van Ooij P, Markl M, Collins JD, Carr JC, Rigsby C, Bonow RO, et al. Aortic valve stenosis alters expression of regional aortic wall shear stress: New insights from a 4-dimensional flow magnetic resonance imaging study of 571 subjects. J Am Heart Assoc. 2017;6(9):1-13. doi:10.1161/JAHA.117.005959

27. Klein S, Staring M, Murphy K, Viergever MA, Pluim JPW. Elastix: A toolbox for intensity-based medical image registration. IEEE Trans Med Imaging. 2010;29(1):196-205. doi:10.1109/TMI.2009.2035616

28. Flores FG, Oñate E. Improvements in the membrane behaviour of the three node rotation-free BST shell triangle using an assumed strain approach. Comput Methods Appl Mech Eng. 2005;194(6-8):907-932. doi:10.1016/j. cma.2003.08.012 




\section{Part IV}

Hemodynamic impact of graft repair 


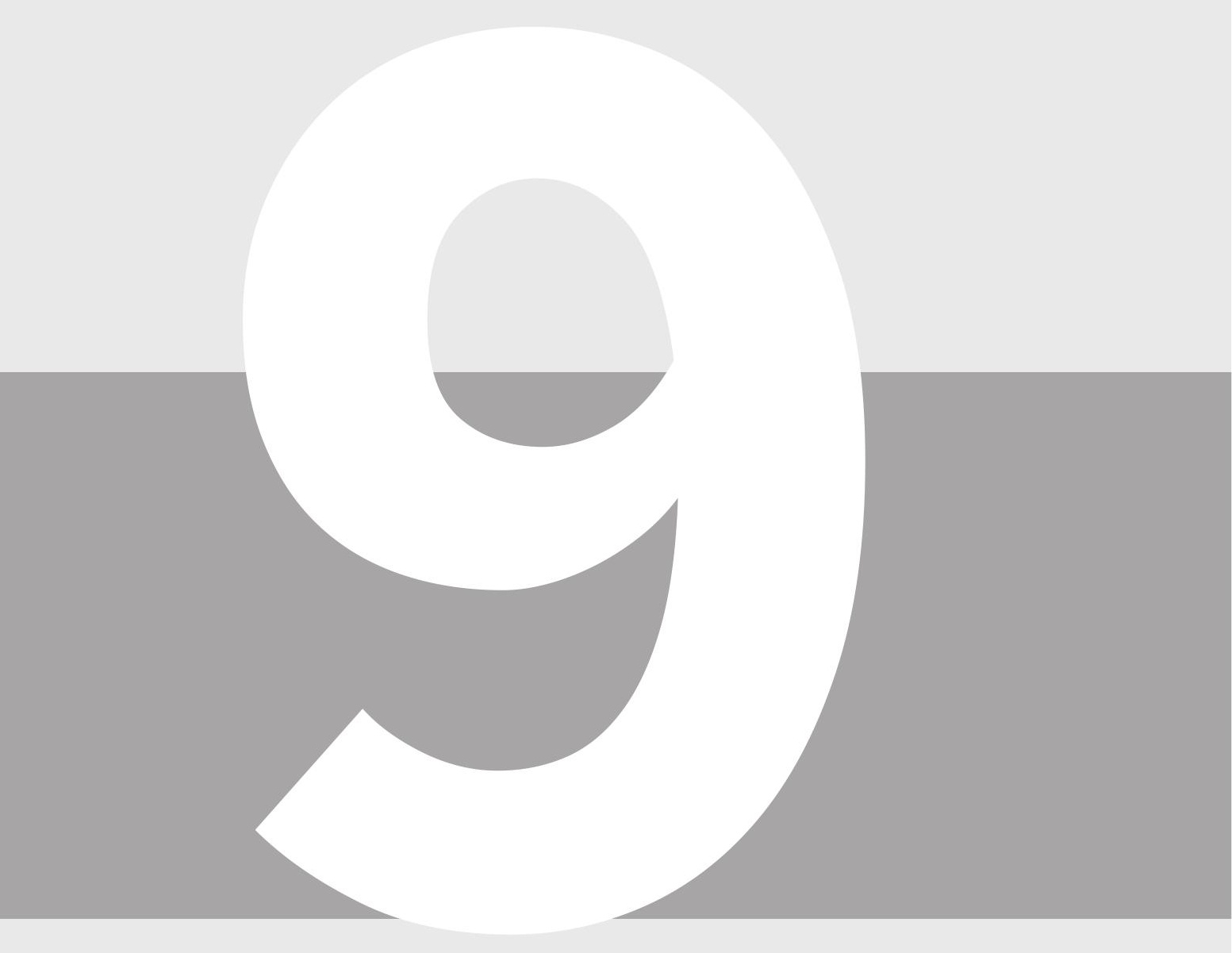




\title{
Left ventricular remodeling following aortic root and ascending aneurysm repair
}

\author{
Ignas B. Houben, ${ }^{1,2} \mathrm{MD}$, \\ Angel K.Y. Chu, ${ }^{3,4} \mathrm{MS}$, \\ Nicholas S. Burris, ${ }^{5} \mathrm{MD}$, \\ Bo Yang, ${ }^{1} \mathrm{MD}, \mathrm{PhD}$, \\ Karen M. Kim, ${ }^{1}$ MD, \\ Shinichi Fukuhara, ${ }^{1} \mathrm{MD}$, \\ Joost A. van Herwaarden, ${ }^{2} \mathrm{MD}, \mathrm{PhD}$ \\ David A. Nordsletten, ${ }^{1,6} \mathrm{PhD}$, \\ C. Alberto Figueroa, ${ }^{3,6} \mathrm{PhD}$, \\ Himanshu J. Patel, ${ }^{1}$ MD.
}

${ }^{1}$ Department of Cardiac Surgery, Frankel Cardiovascular Center, University of Michigan, Ann Arbor, United States ${ }^{2}$ Department of Vascular Surgery, Utrecht University, Utrecht, The Netherlands ${ }^{3}$ Department of Vascular Surgery, Frankel Cardiovascular Center, University of Michigan, Ann Arbor, United States ${ }^{4}$ Department of Computational Medicine and Bioinformatics, University of Michigan, Ann Arbor, United States ${ }^{5}$ Department of Radiology, University of Michigan, Ann Arbor, United States 6Department of Biomedical Engineering, University of Michigan, Ann Arbor, United States Under revision at Journal of Thorac Cardiovasc Surg 


\section{GLOSSARY OF ABBREVIATIONS}

OMT = Optimal medical treatment

$\mathrm{LV}=$ Left ventricle

$\mathrm{ECG}=$ Electrocardiogram

CTA = Computed tomography angiography

Al = Aortic insufficiency

AS $=$ Aortic stenosis

BAV = Bicuspid aortic valve

CTD = Connective tissue disease

AVR = Aortic valve replacement

MVR = Mitral valve replacement

BSA = Body surface area

LVMI = Left ventricular myocardial index

$\mathrm{CCC}=$ Concordance correlation coefficient

AHD = Antihypertensive drug

ACE = Angiotensin-converting enzyme

$\mathrm{CCB}=$ Calcium-channel blocker

ARB = Angiotensin-receptor blocker

$\mathrm{BB}=$ Bèta-blocker 


\section{ABSTRACT}

\section{Objective}

Adverse left ventricular remodeling due to a mismatch between stiffness of native aortic tissue and current polyester grafts seems underestimated. This study was conducted to evaluate the impact of proximal aortic replacement on adverse remodeling of the left ventricle (LV).

\section{Methods}

All aortic root and ascending aortic aneurysm patients were identified ( $n=2001,2006$ 2018). The study cohort consisted of a subset of patients ( $n=98$ ) with two or more ECGgated CT angiograms, but without concomitant aortic valve disease or bicuspid aortic valve, connective tissue disease, acute aortic syndrome or prior history of aortic repair or mitral valve surgery. LV myocardial mass was measured from CT data and indexed to body surface area (LVMI). The study cohort was divided into a surgery group $(n=47)$ and a control group; optimal medical therapy group (OMT, $\mathrm{n}=51$ ).

\section{Results}

The mean age was $60 \pm 11$ years ( $80 \%$ male). Bèta-blocker use was significantly more frequent in the surgery group $(p<0.001)$. All other antihypertensive drug use was significantly more frequent in the OMT group $(p<0.044)$. The average follow-up was $11.6 \pm 5.9$ months. Average LVMI at baseline was similar in both groups $(p=0.934)$. LVMI increased significantly in the surgery group compared to the OMT group $\left(3.7 \pm 4.1 \mathrm{~g} / \mathrm{m}^{2}\right.$ vs. $0.6 \pm$ $\left.4.4 \mathrm{~g} / \mathrm{m}^{2}, \mathrm{p}=0.001\right)$, which was maintained with multivariable linear regression analysis.

\section{Conclusions}

Proximal aortic repair with polyester grafts lead to early LV mass increase, potentially causing adverse cardiac remodeling. Larger cohort studies and extended follow-up are needed to evaluate the long-term impact of current technology. 
Hemodynamic impact of graft repair

\section{CENTRAL MESSAGE}

Proximal aortic stiffening after open graft repair may lead to adverse early left ventricular myocardial mass increase.

\section{PERSPECTIVE STATEMENT}

We present early data regarding the impact of proximal aortic replacement on left ventricular remodeling. Using ECG-gated time interval CTA analysis, we found that polyester graft replacement results in increases in left ventricular mass possibly as a result of acute aortic stiffening and loss of Windkessel function.

\section{Central picture}

Baseline

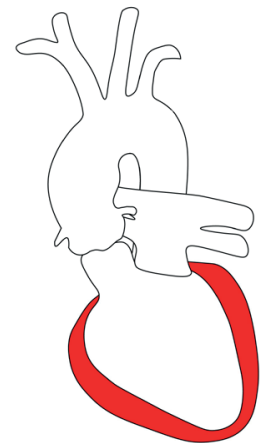

$\operatorname{LVMI}=54.2 \mathrm{~g} / \mathrm{m}^{2}$

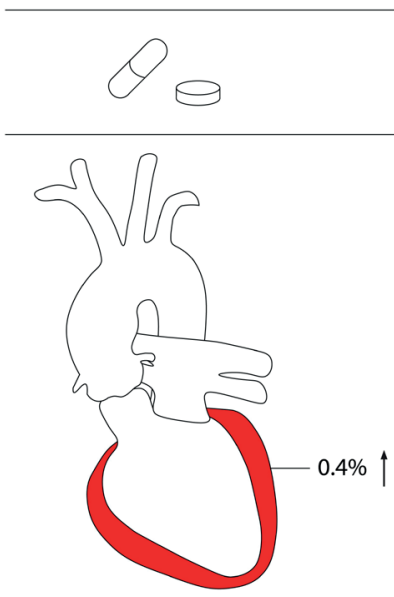

$\operatorname{LVMI}=54.4 \mathrm{~g} / \mathrm{m}^{2}$

$\mathrm{n}=51$

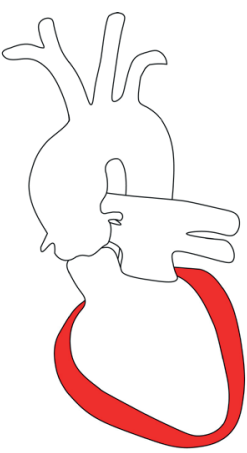

$\operatorname{LVMI}=54.9 \mathrm{~g} / \mathrm{m}^{2}$
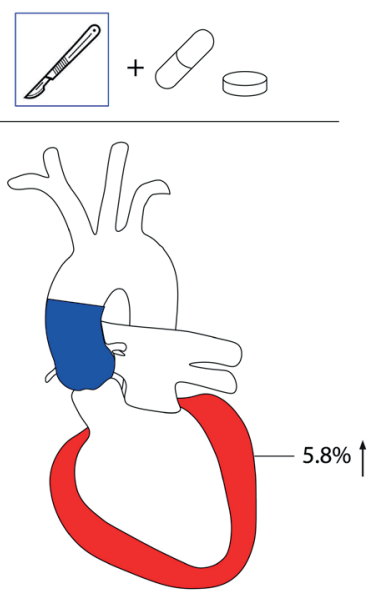

$\operatorname{LVMI}=58.1 \mathrm{~g} / \mathrm{m}^{2}$

$\mathrm{n}=47$

Schematic summary of the study, depicting the measured left ventricular volume in both proximal aortic dilation groups. The group receiving optimal medical treatment only $(n=51)$ showed no significant overall left ventricular mass index change (LVMI, left ventricular mass indexed for body surface area), whereas the group receiving surgery with optimal medical treatment $(n=47)$ revealed significant LVMI increase $(p=0.001)$. 


\section{INTRODUCTION}

The most frequent location of thoracic aortic aneurysms (TAA) occurs in the ascending. ${ }^{1}$ While optimal medical therapy (OMT) revolving around aggressive blood pressure management can prevent complications, definitive management involves open surgery when threshold diameters are reached. ${ }^{2}$ The proximal aorta is the most compliant segment of the aorta and is a large contributor to the Windkessel effect (i.e., the elastic reservoir function of large vessels). ${ }^{3}$ This effect allows for a reduction in left ventricular impedance and continued forward flow in diastole. It has been previously reported that dilation of the ascending aorta is correlated to an increase in left ventricular (LV) mass and a change in left ventricular function. ${ }^{4}$ This observation is likely due to altered ventricular-arterial coupling (VAC) in TAA secondary to loss of elastin and collagen remodeling, resulting in aortic stiffening and dilation and subsequent increase in LV afterload. ${ }^{4,5}$

Current guidelines suggest that ascending aortic diameter of $>50-55 \mathrm{~mm}$ or total diameter growth $>5-10 \mathrm{~mm} /$ year be used as a threshold for surgical repair to prevent rupture. ${ }^{2,6}$ The gold standard for treatment of ascending TAA is open surgical repair with synthetic graft material, commonly made of polyester. These synthetic grafts have a radial stiffness at normal blood pressure values that is approximately 50 times higher than the radial stiffness of native aortic tissue, and can potentially increase left ventricular afterload and subsequently, the left ventricular mass. ${ }^{7-10}$ van Bakel et al. suggested a clinically measurable effect of stiff synthetic endograft on the LV in a small cohort of 8 patients after descending aortic aneurysm repair. ${ }^{11}$ Such effects on the LV may be larger than attributable degenerative changes in the aortic wall, as the change in central stiffness with ascending graft replacement is of higher magnitude and occurs more abruptly. In this study, we attempt to evaluate the effects of synthetic graft repair on left ventricular mass in patients with ascending TAA undergoing open repair with synthetic grafts. We hypothesized that the LV mass increase is larger in patients undergoing synthetic graft repair versus patients treated with OMT only.

\section{METHODS}

Approval for this retrospective study was obtained from our local institutional review board (Protocol number HUM00169089, approved on 09/25/2019), the need for patient consent was waived.

\section{Patient selection}

The Adult Cardiac Surgery database at the University of Michigan Frankel Cardiovascular Center was retrospectively queried for all patients presenting with aortic root and ascending aortic aneurysms between January 2006 and January 2019 (n=2001). Specifi- 
cally, patients were identified via the Society of Thoracic Surgeons (STS) database and our institutional Electronic Medical Record Search Engine (EMERSE). ${ }^{12}$ Patients having at least two electrocardiographically (ECG)-gated computed tomography angiography (CTA) scans and a significant aortic root or ascending aortic dilation with a diameter of at least $40 \mathrm{~mm}$, were included. Patients were excluded for having 1) a history of hemodynamically significant (moderate or severe) aortic insufficiency (AI) or aortic stenosis (AS), 2) bicuspid aortic valve (BAV), 3) connective tissue disease (CTD), 4) any prior acute aortic syndrome or thoracic aortic repair, 5) prior aortic or mitral valve repair or replacement (AVR or MVR), 6) index repair that included AVR or MVR, or 7) a CTA follow-up interval of $<4$ months. After exclusion, 140 patients were selected for further review. After review, an additional 42 patients were excluded based on poor quality CTA data (Figure 1). The population was divided into a treatment group, receiving open valve-sparing root repair and/or ascending repair with OMT, and a control group, receiving OMT only. These will be further referred to as the surgery group and the OMT group.

Figure 1. Inclusion and exclusion chart

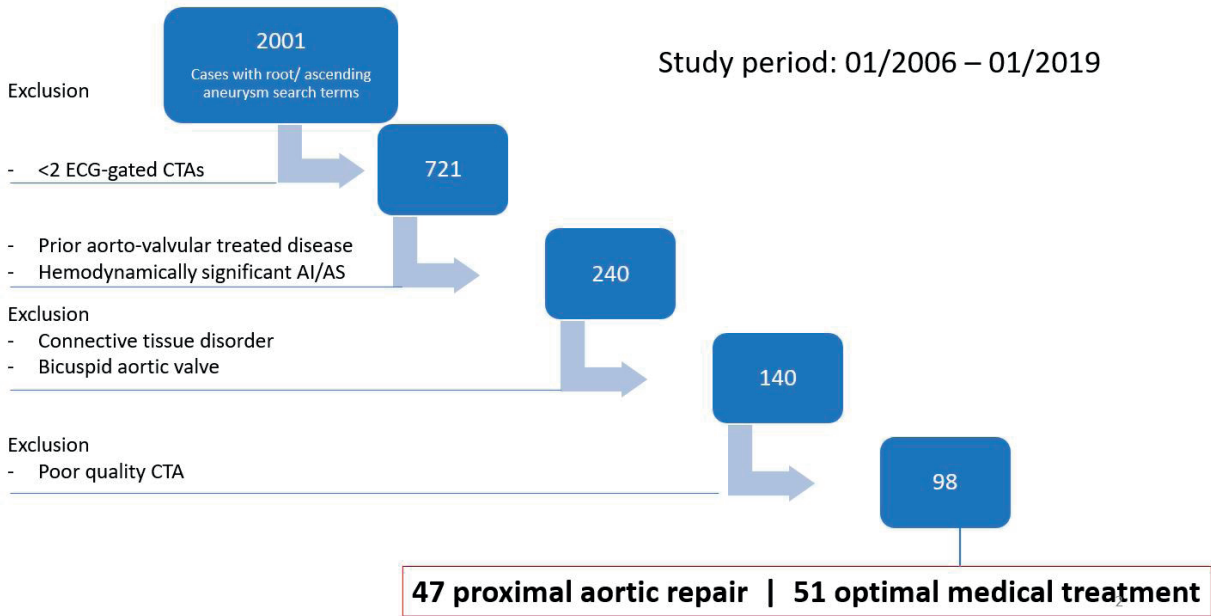

\section{Clinical data}

Clinical data was gathered from the electronic medical records including: brachial blood pressures at baseline and follow-up during clinic visits, maximal proximal (root or ascending) aortic diameter at baseline and follow-up from radiologic reports, antihypertensive drugs (AHD) at baseline and follow-up, body surface area (BSA) at baseline and follow-up, and history of hypertension, dyslipidemia, diabetes mellitus, coronary artery disease and tobacco use. For AHD, reports on five type of drugs were collected: diuretics, angiotensin-converting enzyme (ACE )-inhibitors, calcium-channel blockers (CCB), angiotensin-receptor blockers (ARB) and Bèta-blockers (BB). 


\section{Imaging data}

CTA examinations were acquired on a multi-slice scanner after intravenous injection of $120 \mathrm{~mL}$ iopamidol intravenous contrast (Isoview 370, Bracco Diagnostics, Milan, Italy) and prospective reconstruction was performed ( $75 \%$ of the R-R interval). The CTA image data were analyzed using automatic image processing tools in the software package Vitrea Core (Vital Images Inc., Product Version 6.9.1, Minnetonka, MN, USA). All measurements of myocardial mass were performed by two observers, IBH and AKYC, using a semi-automated delineation of the myocardial volume between the endo- and epicardial borders, multiplied by a tissue density of $1.055 \mathrm{gr} / \mathrm{mL}$ (Figure 2). LV myocardial mass was indexed to body surface area, yielding left ventricular mass index (LVMI) in g/m2. In the surgery group, the baseline scan was defined as the most recent pre-operative CTA.

A random selection of 40 cases was assessed twice by each observer to determine intra- and interobserver agreement of LV mass measurements. For intraobserver measurements, an interval between measurements of at least two weeks was followed to minimize recall.

Figure 2. Measurement methods

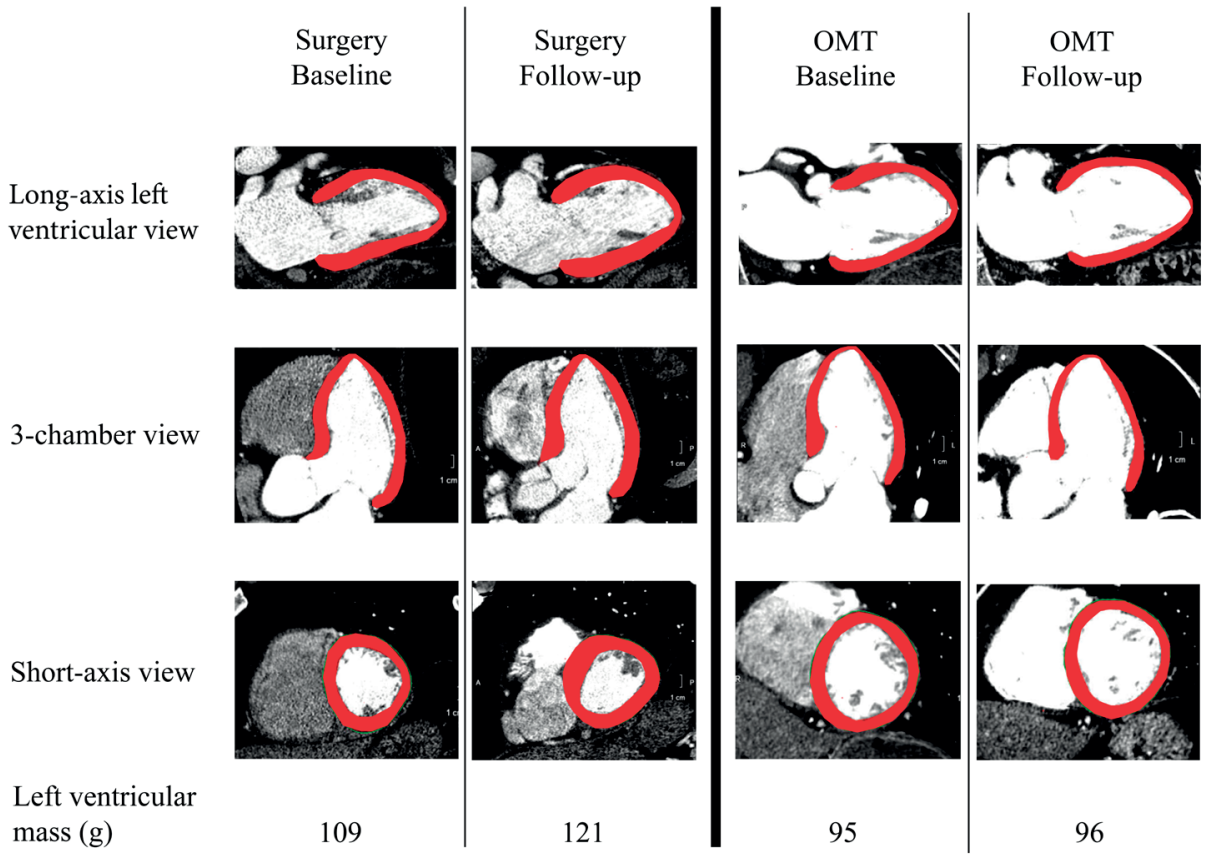

LV mass measurements were taken using semi-automatic image processing tools in Vitrea (Vital Images Inc., Product Version 6.9.1, Minnetonka, MN, USA). 


\section{Statistical analysis}

Continuous data was presented as mean \pm SD and categorical data was presented as frequency and percentage. If a variable had more than $10 \%$ missing data, the amount was specified in the tables. Normality of the distribution of the data was tested using the Shapiro-Wilk test. Intra- and interobserver agreement were analyzed using a concordance correlation coefficient (CCC) and graphically depicted using Bland-Altman plots. ${ }^{13}$ CCC values < 0.90 were considered "poor agreement", between 0.90-0.95 "moderate", 0.95-0.99 "substantial" and >0.99 "almost perfect" as described by Lin et al. ${ }^{14}$ Differences between clinical and imaging data between groups was assessed using chi-square analysis for categorical data and t-test for continuous data. Multiple linear regression analysis was performed to identify independent predictors of change in LVMI amongst a group of potential confounders. Statistical significance was defined as $p<0.05$. Analysis of the data was performed using SPSS Statistics 26 (IBM, Armonk, NY) and R3.6.2 (R Core Team, 2019).

\section{RESULTS}

\section{Baseline characteristics}

98 patients were included for analysis, 47 in the surgical group and 51 in the OMT group. Overall, 78 patients ( $80 \%$ ) were male and mean age was $60 \pm 11$ years. No significant differences between groups were found in sex, age, hypertension, dyslipidemia, diabetes mellitus, coronary artery disease and tobacco use. BB use was more frequent in the surgery group ( $89 \%$ vs. $57 \%, p<0.001$ ) whereas ACE/ARB, diuretic and CCB use was more frequent in the OMT group. There was a significant baseline difference in diastolic pressure between the surgery group and the OMT group ( 81 vs $77 \mathrm{mmHg}, \mathrm{p}=0.046$ ). Patient characteristics are detailed in Table 1 . The distribution of dilation (diameter $>40 \mathrm{~mm}$ ) along the proximal aorta in our population was as follows: $20 \%$ isolated aortic root dilation, $34 \%$ isolated mid-ascending aortic dilation and $46 \%$ both aortic root and ascending aortic dilation. The surgery group had significantly higher baseline diameters at the levels of the root, sinotubular junction, and the mid ascending aorta. No differences were found in baseline left ventricular mass between the surgery and the OMT group. Radiographic characteristics are detailed in Table 2.

\section{Follow-up characteristics}

The CT follow-up interval for the surgery group was $9.1 \pm 4.0$ months, and for the OMT group was $13.7 \pm 6.3$ months, $p<0.001$. The average aortic growth in the OMT group at aortic root level was $0.5 \pm 1.6 \mathrm{~mm}$ and at the mid ascending aortic level it was $0.1 \pm 1.2$ $\mathrm{mm}$. 
Table 1 - Demographics and comorbidities

\begin{tabular}{|c|c|c|c|c|}
\hline Baseline characteristics & Overall & $\begin{array}{l}\text { Optimal medical } \\
\text { treatment + } \\
\text { Surgery }\end{array}$ & $\begin{array}{l}\text { Optimal } \\
\text { medical } \\
\text { treatment }\end{array}$ & p-value \\
\hline & $(n=98)$ & $(n=47)$ & $(n=51)$ & \\
\hline Age, years $\pm S D$ & $59.9 \pm 10.5$ & $60.1 \pm 10.5$ & $59.7 \pm 10.5$ & 0.821 \\
\hline Sex, male (\%) & $78(80)$ & $38(81)$ & $40(78)$ & 0.963 \\
\hline $\mathrm{BSA}, \mathrm{m} 2 \pm \mathrm{SD}$ & $2.09 \pm 0.25$ & $2.09 \pm 0.21$ & $2.09 \pm 0.29$ & 0.915 \\
\hline $\mathrm{BMI} \pm \mathrm{IQR}$ & $28.7(26.2-31.3)$ & $28.9(26.2-30.7)$ & $28.3(26.2-30.4)$ & $0.548^{\wedge}$ \\
\hline Aortic root dilation only, $\mathrm{n}(\%)^{\star}$ & $20(20)$ & $10(21)$ & $10(20)$ & 0.838 \\
\hline Hypertension & $66(68)$ & $34(72)$ & $32(63)$ & 0.312 \\
\hline Coronary artery disease & $17(17)$ & $12(26)$ & $5(10)$ & 0.074 \\
\hline Diabetes mellitus & $7(7)$ & $4(9)$ & $3(6)$ & $\dagger 0.717 \dagger$ \\
\hline Chronic kidney disease & $5(5)$ & $2(4)$ & $3(6)$ & $0.715 \dagger$ \\
\hline Tobacco use & $38(39)$ & $18(38)$ & $20(39)$ & 0.926 \\
\hline Dyslipidemia & $44(45)$ & $21(45)$ & $23(45)$ & 0.967 \\
\hline Prior cardiovascular repair & $5(5)$ & $4(9)$ & $1(2)$ & †0.191† \\
\hline Diuretic use & $16(16)$ & $4(9)$ & $12(24)$ & 0.044 \\
\hline ACE-inhibitor use & $22(22)$ & $4(9)$ & $18(35)$ & 0.002 \\
\hline Calcium-channel blocker use & $12(12)$ & $2(4)$ & $10(20)$ & 0.036 \\
\hline Angiotensin-receptor blocker use & $14(14)$ & $2(4)$ & $12(24)$ & 0.006 \\
\hline Beta-blocker use & $71(72)$ & $42(89)$ & $29(57)$ & $<0.001$ \\
\hline Pulse pressure, $\mathrm{mmHg} \pm \mathrm{IQR}$ & $49(40-59)$ & $50.5(40.8-58.8)$ & $48.5(40.2-58.8)$ & $\wedge 0.613$ \\
\hline Systolic blood pressure, $\mathrm{mmHg} \pm \mathrm{SD}$ & $130 \pm 17$ & $133 \pm 16$ & $128 \pm 17$ & 0.115 \\
\hline Diastolic blood pressure, $\mathrm{mmHg} \pm \mathrm{SD}$ & $79 \pm 9$ & $81 \pm 10$ & $77 \pm 8$ & 0.046 \\
\hline
\end{tabular}

* No ascending dilation involvement reported between the sinotubular junction and the innominate artery

$\dagger$ Fisher's exact test;^ Mann-Whitney U test

Abbreviations: $\mathrm{IQR}=$ interquartile range, $\mathrm{SD}=$ standard deviation, $\mathrm{BSA}=$ body surface area, $\mathrm{BMI}=$ body mass index, ACEinhibitor = angiotensin-converting enzyme inhibitor, LVM = Left Ventricular Mass, LVMI = Left Ventricular Mass Index, LVMI Change $=$ the difference between Left Ventricular Mass Index at baseline and during follow-up.

LVMI increased significantly in the surgery group compared to the OMT group $(3.7 \pm 4.1 \mathrm{~g} /$ $\mathrm{m}^{2}$ vs. $0.6 \pm 4.4 \mathrm{~g} / \mathrm{m}^{2}, \mathrm{p}=0.001$, Figure 3 ). There was a trend towards higher pulse pressure change during follow-up in the surgery group, but the difference was not statistically significant ( $3.3 \pm 12.6$ vs. $-1.6 \pm 12.6 \mathrm{mmHg}, \mathrm{p}=0.063$, Figure 3$)$.

\section{Bivariable and Multivariable analysis}

Prior to conducting the multivariable analysis, we examined bivariable correlations between LVMI change and clinical and radiographic independent variables. Bivariable analysis was performed for our entire population ( $n=98)$, showing a positive correlation between male sex and LVMI change $(R=0.208, p=0.039)$. Subsequently, bivariable correlation was examined for each treatment group. In the surgery group, a positive correlation 
Hemodynamic impact of graft repair

Table 2 - Radiographic characteristics

\begin{tabular}{|c|c|c|c|c|}
\hline Baseline characteristics & Overall & $\begin{array}{c}\text { Optimal medical } \\
\text { treatment + } \\
\text { Surgery }\end{array}$ & $\begin{array}{l}\text { Optimal medical } \\
\text { treatment }\end{array}$ & p-value \\
\hline & $(n=98)$ & $(n=47)$ & $(n=51)$ & \\
\hline Mild aortic insufficiency, $\mathrm{n}(\%)^{*}$ & $28(29)$ & $17(37)$ & $11(22)$ & 0.110 \\
\hline \multicolumn{5}{|c|}{ Baseline aortic diameter, mm median (IQR): } \\
\hline Root & $45(42-50)$ & $49(45-52.5)$ & $43(39.7-46.4)$ & $<0.001^{\wedge}$ \\
\hline Sinotubular junction & $40(37-43)$ & $42(39-44.8)$ & $39(36-41.3)$ & $<0.001^{\wedge}$ \\
\hline Mid-ascending & $44.8(40.2-47)$ & $45(41.6-49.1)$ & $44(40-46)$ & $0.040^{\wedge}$ \\
\hline Overall maximum aortic diameter & $45(41.1-47.7)$ & $46(43-50)$ & $44(40.4-46)$ & $0.002^{\wedge}$ \\
\hline CT scan interval, days median (IQR) & $340(210-392)$ & $242(188-296)$ & $368(344-472)$ & $<0.001^{\wedge}$ \\
\hline \multicolumn{5}{|l|}{ Left ventricular parameters: } \\
\hline $\mathrm{LVM}, \mathrm{g} \pm \mathrm{SD}$ & $114 \pm 27$ & $114 \pm 25$ & $114 \pm 30.0$ & 0.956 \\
\hline LVMI at baseline, $\mathrm{g} / \mathrm{m} 2 \pm \mathrm{SD}$ & $54.3 \pm 9.8$ & $54.4 \pm 9.5$ & $54.2 \pm 10.2$ & 0.934 \\
\hline LVMI change, $\mathrm{g} / \mathrm{m} 2 \pm \mathrm{SD}$ & $2.08 \pm 4.5$ & $3.67 \pm 4.1$ & $0.62 \pm 4.4$ & $<0.001$ \\
\hline
\end{tabular}

*Significant aortic insufficiency was excluded from the dataset, so this group constitutes mild insufficiency versus minimal or no insufficiency

$\wedge$ Mann-Whitney U test

Abbreviations: $\mathrm{IQR}=$ interquartile range, $\mathrm{SD}=$ standard deviation, $\mathrm{CT}=$ computed tomography, $\mathrm{LVM}=$ left ventricular mass, LVMI = left ventricular mass index.

Figure 3. LVMI change and pulse pressure change chart

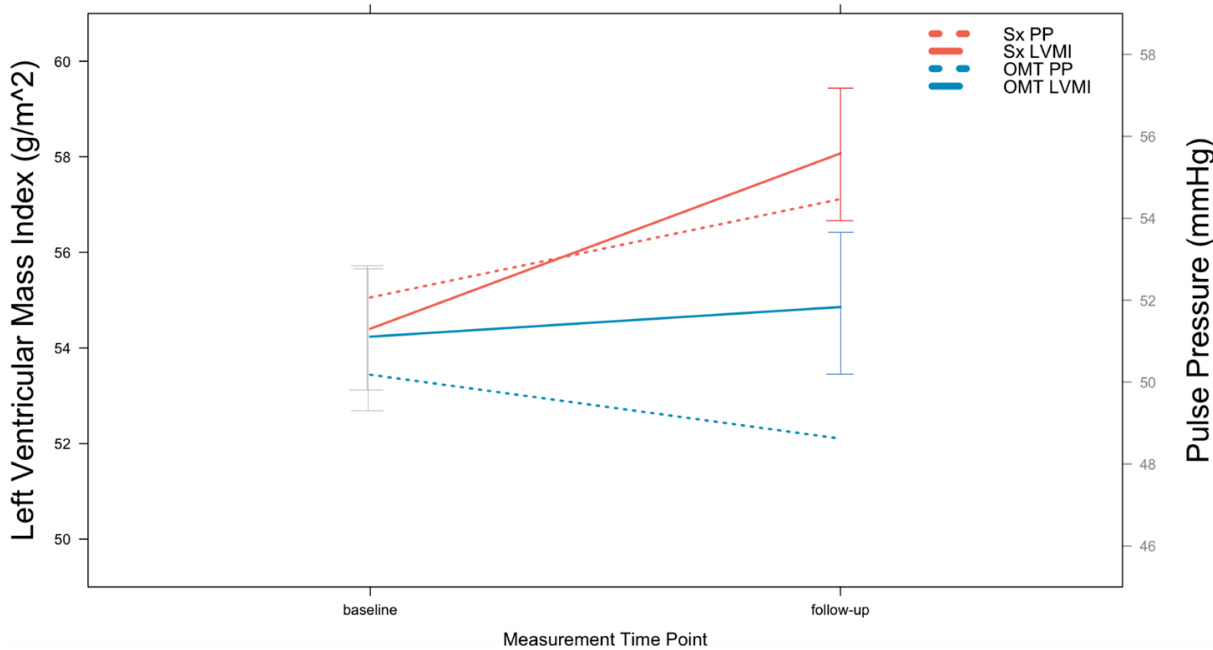

Mean left ventricular mass index at timepoint 1 and 2 for each group, including the standard error bars, showing a significant growth over time for the surgery + OMT group (red) vs the OMT group (blue) ( ${ }^{*} ; p<0.001$; Student's t-test).

was seen between LVMI increase and age $(R=0.343, p=0.018)$. In both the surgery group and the OMT group, CCB use was associated with a positive effect on LVMI change (surgery: $7.2 \mathrm{~g} / \mathrm{m}^{2}$; OMT: $3.5 \mathrm{~g} / \mathrm{m}^{2} ; \mathrm{p}=0.031$ ). However, considering the low frequency of CCB 
and ARB use in the surgery group $(2 / 47,4 \%)$, these variables were not included in the multivariable analysis. The effect of surgery on LVMI in a bivariable analysis was positive with a coefficient of $3.050 \mathrm{~g} / \mathrm{m}^{2}(p=0.001)$. Bivariable analysis is detailed in Table 3.

Table 3 - Univariate regression analysis of LVMI change with baseline clinical parameters among treatment groups

\begin{tabular}{|c|c|c|c|c|c|c|}
\hline \multirow[t]{3}{*}{ Baseline characteristics } & \multicolumn{2}{|c|}{$\begin{array}{l}\text { Overall } \\
(n=98)\end{array}$} & \multicolumn{2}{|c|}{$\begin{array}{l}\text { Optimal medical } \\
\text { treatment + Surgery } \\
\qquad(n=47)\end{array}$} & \multicolumn{2}{|c|}{$\begin{array}{l}\text { Optimal medical } \\
\text { treatment } \\
(n=51)\end{array}$} \\
\hline & \multicolumn{2}{|c|}{ LVMI change, g/m2 } & \multicolumn{2}{|c|}{ LVMI change, g/m2 } & \multicolumn{2}{|c|}{ LVMI change, g/m2 } \\
\hline & Coefficient & p-value & Coefficient & p-value & Coefficient & p-value \\
\hline Age, years & 0.070 & 0.11 & 0.13 & 0.018 & 0.004 & 0.95 \\
\hline BMI & 0.079 & 0.41 & 0.019 & 0.89 & 0.17 & 0.18 \\
\hline Pulse pressure change, $\mathrm{mmHg}$ & 0.016 & 0.67 & -0.024 & 0.62 & 0.005 & 0.92 \\
\hline Maximal aortic diameter, mm & 0.028 & 0.74 & -0.14 & 0.16 & 0.042 & 0.80 \\
\hline Baseline LVMI, g/m2 & -0.081 & 0.083 & -0.93 & 0.15 & -0.074 & 0.23 \\
\hline CT interval, days & -0.001 & 0.64 & -0.001 & 0.82 & 0.004 & 0.21 \\
\hline Sex, male & 2.34 & 0.040 & 2.76 & 0.069 & 1.75 & 0.25 \\
\hline Diuretic at baseline use & 0.84 & 0.49 & 4.065 & 0.035 & 0.57 & 0.70 \\
\hline ACE-inhibitor use & 1.80 & 0.10 & 4.69 & 0.014 & 2.42 & 0.068 \\
\hline Calcium-channel blocker use & 2.16 & 0.18 & 4.41 & 0.29 & 3.53 & 0.039 \\
\hline Angiotensin-receptor blocker use & -1.70 & 0.24 & 3.03 & 0.47 & -0.77 & 0.63 \\
\hline Beta blocker use & 0.30 & 0.77 & -0.71 & 0.72 & -1.30 & 0.31 \\
\hline
\end{tabular}

Abbreviations: $\mathrm{BMI}=$ body mass index, $\mathrm{LVMI}=$ left ventricular mass index, $\mathrm{CT}=$ Computed Tomography, $\mathrm{ACE}$-inhibitor = angiotensin-converting enzyme inhibitor.

Table 4 - Multivariate regression analysis with left ventricular mass index change as the dependent variable.

\begin{tabular}{lcccc} 
Characteristics & Coefficient & $\begin{array}{c}\text { Robust standard } \\
\text { error }\end{array}$ & p-value & $\begin{array}{c}95 \% \text { Confidence } \\
\text { interval }\end{array}$ \\
\hline Age, years & 0.132 & 0.042 & 0.002 & $0.048 ; 0.216$ \\
\hline Sex, male & 3.720 & 1.065 & 0.001 & $1.604 ; 5.837$ \\
\hline BMI & 0.240 & 0.089 & 0.009 & $0.062 ; 0.417$ \\
Diuretic use & 1.967 & 1.102 & 0.078 & $-0.223 ; 4.158$ \\
\hline ACE-inhibitor use & 1.638 & 0.979 & 0.098 & $-0.308 ; 3.584$ \\
Bèta-blocker use & -1.362 & 0.925 & 0.144 & $-3.201 ; 0.476$ \\
\hline Maximal aortic diameter, mm & -0.163 & 0.085 & 0.058 & $-0.332 ; 0.006$ \\
\hline Baseline left ventricular mass index, g/m2 & -0.158 & 0.044 & 0.001 & $-0.246 ;-0.070$ \\
\hline CT interval, days & 0.000 & 0.002 & 0.953 & $-0.005 ; 0.005$ \\
\hline Treatment, surgery & 4.903 & 0.966 & 0.000 & $2.983 ; 6.824$ \\
\hline
\end{tabular}


We performed a multivariable analysis to identify independent predictors of LVMI change amongst the following predictors at baseline: age, sex, BMI, diuretic use, ACEinhibitor use, beta-blocker use, maximal proximal aortic diameter, LVMI and time-interval between CTA studies. On adjusted analysis we identified the following significant predictors of LVMI increase during follow-up: surgery $(\beta=4.903, p<0.001)$, age $(\beta=0.132$, $p=0.002)$, sex $(\beta=3.720, p=0.001)$, BMI $(\beta=0.240, p=0.009)$, and baseline LVMI $(\beta=-0.158$, $\mathrm{p}=0.001)$. Multiple linear regression results are shown in Table 4.

\section{DISCUSSION}

This study was conducted to investigate the effect of proximal aortic graft repair on LV mass. We used 3-D ECG gated CTA data to assess LV mass. We found that over the first year of post-operative imaging surveillance, the left ventricle undergoes a significant increase in mass following ascending aortic and/or aortic root graft repair, a relationship that persisted after adjustment for potential confounders. Furthermore, age, male sex, $\mathrm{BMI}$ and baseline LVMI were also found to be significant predictors for LVMI increase in our cohort. Finally, although not statistically significant $(p=0.06)$, the trends in increased pulse pressure observed in the surgery group may support the hemodynamic consequences of acutely induced aortic stiffening.

Stiffening of the proximal aorta is known to have a detrimental effect on cardiovascular health. ${ }^{16,17}$ One of the direct adverse consequences of pathological central stiffening is an increase in afterload and altered ventricular-arterial coupling, potentially leading to development of left ventricular hypertrophy and increased risk of diastolic heart failure. ${ }^{4,18,19}$ Although previous animal studies have described left ventricular mass change following acute aortic stiffening, ${ }^{20,21}$ the phenomenon is not as well described in humans. Increased LVMI following central stiffening has been associated with an increase in the risk of heart failure. ${ }^{22}$ These studies however, demonstrate the adverse effects of central arterial stiffening in a chronic process, where loss of compliance due to elastin loss and collagen structure malformations cause hypertension, and further age-related arterial stiffening. Conversely, our study describes the consequences of an acute change of central artery stiffness with a vastly different order of magnitude compared to that seen with aging. In the short term, the hemodynamics consequences of such a rapid change in proximal aortic stiffness may be significant, as suggested by our data and previously described in animal models. ${ }^{20,21,23}$

Stiff polyester grafts have been utilized as aortic replacement substitutes for decades. The recent emergence of endovascular aortic repair has been used to reduce morbidity of the operative intervention. Their constructs suggest that their compliance may be 
better than polyester graft material. However, a recent computational fluid dynamic and imaging study from our group suggested that even thoracic endovascular aortic repair may result in an increase in left ventricular mass due to its effects on aortic compliance. ${ }^{11}$

The positive correlation between age and LVMI increase also seen in the surgery group suggests that patients with advanced age may be at highest risk for adverse remodeling after open TAA repair. Age is known to be associated with a relative left ventricular mass increase, manifested as concentric hypertrophy, as a result of vascular stiffening. ${ }^{24,25}$ Furthermore, age has also been associated with unfolding of the aortic arch, decreased aortic distensibility and increased arch pulse wave velocity and central aortic pressures. ${ }^{24}$ The potential additive late consequences of aortic stiffening from aortic graft repair in older vs. younger patients may need further study.

Additionally, we identified male sex as a predictor of LVMI increase in the regression analysis. Having a higher incidence of LVMI increase in male patients and in older patients has been previously described. In a large population cardiovascular MRI study, 741 subjects were analyzed, revealing a high degree of concentric hypertrophy in males. ${ }^{26}$ Moreover, a strong positive correlation was found between BMI and LV mass, which underlines the potential confounding effect of BMI on LVMI increase found in our analysis. Last, our findings in the multivariable regression analysis are suggestive of a potential negative effect of baseline LVMI on LVMI increase. While the exact mechanism for this observation is unclear, it seems reasonable that patients with higher baseline LVMI may have less potential for further hypertrophy, supporting the theory of left ventricular hypertrophy reaching a plateau, which has been previously described. ${ }^{23}$

\section{Limitations}

This study is limited by the sample size, different time intervals of follow-up imaging, baseline differences in antihypertensive medication use and its retrospective nature. The time intervals were longer for the OMT group. While adjusted analysis can ameliorate some of these effects, a randomized study which would be the gold standard approach is unlikely to be performed for this disease. As such, future larger study cohorts studied prospectively will be needed to validate the findings observed in this study.

\section{CONCLUSION}

Short-term LVMI in proximal aortic aneurysm patients significantly increases after surgical repair, compared to patients treated with OMT only. To our knowledge, this was the first study to assess the isolated effect of ascending aortic stiffening on afterload and subsequent adverse LV remodeling using ECG-gated CTA data. As aortic surgery is 
performed for a life expectancy benefit, and must be performed with a substitute for the repaired aortic segment, further studies should be considered to evaluate the competing risks of aortic related mortality against risks of long term graft induced aortic stiffening and potentially modify current thresholds for aortic intervention. Finally, alternative aortic replacements which are more compliant yet maintain durability seen with current polyester technology should be developed.

\section{Acknowledegments}

The authors gratefully acknowledge the generous support from the David Hamilton Fund and the Phil Jenkins Breakthrough Fund in Cardiac Surgery at the University of Michigan Frankel Cardiovascular Center. 
Left ventricular remodeling following aortic root and ascending aneurysm repair

\section{REFERENCES}

1. Isselbacher EM. Thoracic and abdominal aortic aneurysms. Circulation. 2005;111(6):816-828. doi:10.1161/01. CIR.0000154569.08857.7A

2. Erbel R, Aboyans V, Boileau C, Bossone E, Di Bartolomeo R, Eggebrecht H, et al. 2014 ESC guidelines on the diagnosis and treatment of aortic diseases. Russ J Cardiol. 2015;123(7):7-72. doi:10.15829/1560-4071-2015-077-72

3. Krüger T, Veseli K, Lausberg H, Vöhringer L, Schneider W, Schlensak C. Regional and directional compliance of the healthy aorta: An ex vivo study in a porcine model. Interact Cardiovasc Thorac Surg. 2016;23(1):104-111. doi:10.1093/icvts/ivw053

4. Boonyasirinant T, Rajiah P, Setser RM, Lieber ML, Lever HM, Desai MY, et al. Aortic Stiffness Is Increased in Hypertrophic Cardiomyopathy With Myocardial Fibrosis. Novel Insights in Vascular Function From Magnetic Resonance Imaging. J Am Coll Cardiol. 2009;54(3):255-262. doi:10.1016/j.jacc.2009.03.060

5. Humphrey JD, Tellides G. Central artery stiffness and thoracic aortopathy. Am J Physiol - Hear Circ Physiol. 2019;316(1):H169-H182. doi:10.1152/ajpheart.00205.2018

6. Hiratzka LF, Bakris GL, Beckman JA, Bersin RM, Carr VF, Casey DE, et al. 2010 ACCF/AHA/AATS/ACR/ASA/SCA/ SCAI/SIR/STS/SVM Guidelines for the Diagnosis and Management of Patients with Thoracic Aortic Disease. Anesth Analg. 2010;111(2):279-315. doi:10.1016/j.jacc.2010.02.010

7. Ioannou C V., Stergiopulos N, Katsamouris AN, Startchik I, Kalangos A, Licker MJ, et al. Hemodynamics induced after acute reduction of proximal thoracic aorta compliance. Eur J Vasc Endovasc Surg. 2003;26(2):195-204. doi:10.1053/ejvs.2002.1917

8. Tremblay D, Zigras T, Cartier R, Leduc L, Butany J, Mongrain R, et al. A Comparison of Mechanical Properties of Materials Used in Aortic Arch Reconstruction. Ann Thorac Surg. 2009;88(5):1484-1491. doi:10.1016/j.athoracsur.2009.07.023

9. Roccabianca S, Figueroa CA, Tellides G, Humphrey JD. Quantification of regional differences in aortic stiffness in the aging human. J Mech Behav Biomed Mater. 2014;29:618-634. doi:10.1016/j.jmbbm.2013.01.026

10. Spadaccio C, Nappi F, Al-Attar N, Sutherland FW, Acar C, Nenna A, et al. Old Myths, New Concerns: the LongTerm Effects of Ascending Aorta Replacement with Dacron Grafts. Not All That Glitters Is Gold. J CardiovasC Transl Res. 2016;9(4):334-342. doi:10.1007/s12265-016-9699-8

11. van Bakel TM, Arthurs CJ, Nauta FJ, Eagle KA, van Herwaarden JA, Moll FL, et al. Cardiac remodeling following thoracic endovascular aortic repair for descending aortic aneurysms. Eur J Cardio-Thoracic Surg. 2018;0:1-10. doi:10.1016/0038-1098(79)91043-3

12. Hanauer DA, Mei Q, Law J, Khanna R, Zheng K. Supporting information retrieval from electronic health records: A report of University of Michigan's nine-year experience in developing and using the Electronic Medical Record Search Engine (EMERSE). J Biomed Inform. 2015;55:290-300. doi:10.1016/j.jbi.2015.05.003

13. Lin LI-K. A Concordance Correlation Coefficient to Evaluate Reproducibility. Vol 45.; 1989. doi:10.2307/2532051

14. Lin LI, McBride G, Bland JM, Altman DG. A Proposal for Strength-of-Agreement Criteria for Lin's Concordance Correlation Coefficient. Vol 45. Hamilton; 2005. doi:10.2307/2532051

15. Fuchs A, Mejdahl MR, Kühl JT, Stisen ZR, Nilsson EJP, Køber LV, et al. Normal values of left ventricularmass and cardiac chamber volumes assessed by 320-detector computed tomography angiography in the Copenhagen General Population Study. Eur Heart J Cardiovasc Imaging. 2016;17(9):1009-1017. doi:10.1093/ehjci/jev337

16. Redheuil A, Wu CO, Kachenoura N, Ohyama Y, Yan RT, Bertoni AG, et al. Proximal aortic distensibility is an independent predictor of all-cause mortality and incident CV events: The MESA study. J Am Coll Cardiol. 2014;64(24):2619-2629. doi:10.1016/j.jacc.2014.09.060

17. Kingwell BA, Waddell TK, Medley TL, Cameron JD, Dart AM. Large artery stiffness predicts ischemic threshold in patients with coronary artery disease. J Am Coll Cardiol. 2002;40(4):773-779. doi:10.1016/S0735-1097(02)020090

18. Johnson K, Oparil S, Davis BR, Tereshchenko LG. Prevention of Heart Failure in Hypertension-Disentangling the Role of Evolving Left Ventricular Hypertrophy and Blood Pressure Lowering: The ALLHAT Study. J Am Heart Assoc. 2019;8(8). doi:10.1161/JAHA.119.011961 
Hemodynamic impact of graft repair

19. Williams B, Mancia G, Spiering W, Rosei EA, Azizi M, Burnier M, et al. 2018 ESC/ESH Guidelines for themanagement of arterial hypertension. Eur Heart J. 2018;39(33):3021-3104. doi:10.1093/eurheartj/ehy339

20. Kelly RP, Tunin R, Kass DA. Effect of reduced aortic compliance on cardiac efficiency and contractile function of in situ canine left ventricle. Circ Res. 1992;71(3):490-502. doi:10.1161/01.RES.71.3.490

21. Morita S, Asou T, Kuboyama I, Harasawa Y, Sunagawa K, Yasui H. Inelastic vascular prosthesis for proximal aorta increases pulsatile arterial load and causes left ventricular hypertrophy in dogs. J Thorac Cardiovasc Surg. 2002;124(4):768-774. doi:10.1067/mtc.2002.124244

22. Levy D, Garrison RJ, Savage DD, Kannel WB, Castelli WP. Prognostic implications of echocardiographically determined left ventricular mass in the framingham heart study. N Engl J Med. 1990;322(22):1561-1566. doi:10.1056/ NEJM199005313222203

23. Bjørnstad JL, Skrbic B, Sjaastad I, Bjørnstad S, Christensen G, Tønnessen T. A mouse model of reverse cardiac remodelling following banding-debanding of the ascending aorta. Acta Physiol. 2012;205(1):92-102. doi:10.1111/j.1748-1716.2011.02369.x

24. Redheuil A, Yu WC, Mousseaux E, Harouni AA, Kachenoura N, Wu CO, et al. Age-related changes in aortic arch geometry: Relationship with proximal aortic function and left ventricular mass and remodeling. J Am Coll Cardiol. 2011;58(12):1262-1270. doi:10.1016/j.jacc.2011.06.012

25. Cheng S, Fernandes VRS, Bluemke DA, McClelland RL, Kronmal RA, Lima JAC. Age-related left ventricular remodeling and associated risk for cardiovascular outcomes the multi-ethnic study of atherosclerosis. Circ Cardiovasc Imaging. 2009;2(3):191-198. doi:10.1161/CIRCIMAGING.108.819938

26. Rider OJ, Lewandowski A, Nethononda R, Petersen SE, Francis JM, Pitcher A, et al. Gender-specific differences in left ventricular remodelling in obesity: Insights from cardiovascular magnetic resonance imaging. Eur Heart J. 2013;34(4):292-299. doi:10.1093/eurheartj/ehs341 




\section{Part V}

Discussion 


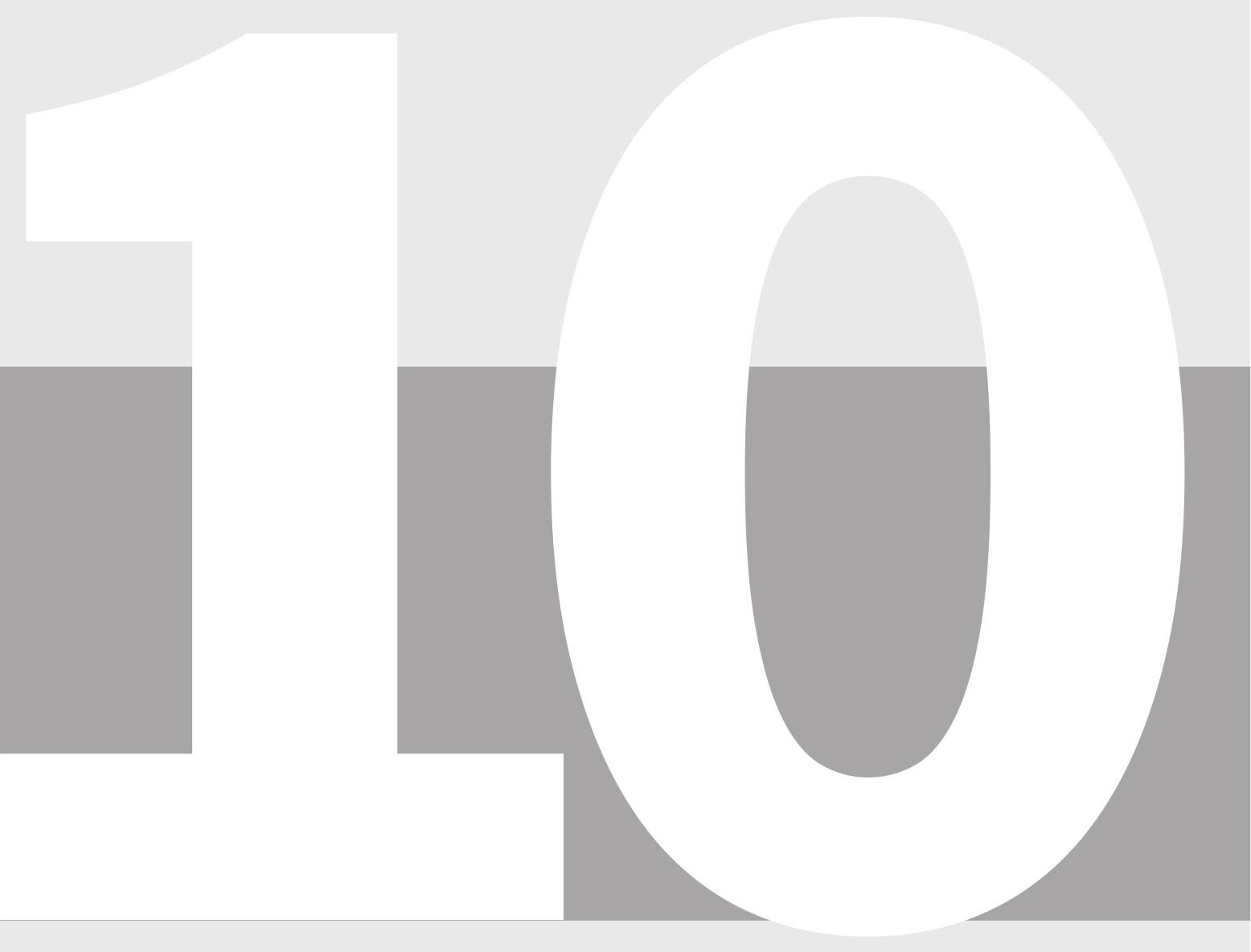


Discussion 



\section{DISCUSSION}

Thoracic aortic pathology management has severely improved over the last three decades with a short- ( $\leq 6$ months) and mid-term ( $\leq 5$ years) mortality reduction, following surgical intervention for dissections from $26-31 \%$ to approximately $10-18 \%^{1-5}$ and a reduction following intervention for aneurysms from approximately $10-45 \%$ depending on the acuity, to approximately $5-25 \% .^{5-9}$ The risk stratification in aortic repair has been taken to a higher level by means of providing patient-specific features and prohibitive profiles for specific types of repair. Thoracic endovascular aortic repair (TEVAR) is currently the main applied interventional strategy to address descending thoracic aortic pathologies. ${ }^{9-11}$ However, until now, no randomized controlled trials are performed to compare the old to the new gold standard. ${ }^{12}$ Dedicated thoracic aortic management guidelines introduced by the cardiovascular societies have cited level 2 and 3 evidence to support their judgement. ${ }^{9-11}$ The amount of level 2 and 3 evidence, revealing limited short-term complications after endovascular therapy is staggering. Clearly, rapid translational technical developments in endovascular surgery have surpassed the speed of clinical gold standard scientific research. Moreover, patients treated with endovascular repair are often unfit for open surgery and can therefore not be randomized for trials. Therefore, it is of vital importance to apply a full parametric assessment into endovascular therapy and to investigate its long-term effect.

This thesis strives to build upon earlier evidence to improve our understanding of the imaging and the hemodynamic aspects of endovascular therapy and to evaluate the latest clinical outcomes. In part II the assessment of conventional versus progressive therapy in type A dissection (TAAD) was started, focusing on the complete management and follow-up of the disease rather than limiting to the entry-tear. It continued to discuss our road to minimal-invasive management in the least charted endovascular part of the aorta, the ascending part. In part III, this thesis evaluated the latest challenges in TEVAR of the descending aorta and introduced new multiparametric methods to meet biomechanical understanding of aneurysm and dissection formation. Finally, in part IV, the clinical hemodynamic effect of introducing stiff synthetic material into the aorta is evaluated.

\section{Part II - Current status of type A aortic dissection management}

TAAD remains one of the few aortic pathologies where open surgical repair is still the indisputable gold standard of treatment. ${ }^{9-11}$ Since this type of surgery mostly requires timely and specialized intervention, early survival increased after the introduction of dedicated aortic surgery teams in aortic referral centers. ${ }^{13,14}$ The current 30 -day postoperative survival rate is approximately $80-85 \%$ for all patients who reach the hospital in time and are deemed fit for surgery. ${ }^{3,4,15}$ While there is still some room for immediate 
technical improvement, an important focus should be put on the long-term prediction of complications. With good survival rates, follow-up results have revealed that 1-2 in 10 surviving patients still requires a reintervention to prevent rupture of the residual aortic dissection within approximately 10 years after index the index procedure. ${ }^{16,17} \mathrm{~A}$ better understanding of this could provide a potential improvement in outcome and more importantly, improve patient quality of life (QoL). Retrospective quality of life (QoL) assessments in patients following TAAD have been carried out in many single center cohorts. Age is a well-known significant risk factor for early mortality in TAAD repaired patients and would intuitively appear to affect the QoL. However, multiple studies found that, in the early survivors, QoL scores among varying age groups were similar during follow-up. ${ }^{18-20}$ Octogenarians seem to lose a survival benefit of surgical vs conservative TAAD repair after 3 to 5 years. ${ }^{21-23}$ However, considering that the average life expectancy in most countries is no higher than 83 years, the survival benefit in octogenarians seems less urgent than the QoL benefit. Reintervention rates may have a prominent role in these QoL assessments, as every re-sternotomy may introduce a considerable rehabilitation period. No study has yet described QoL in TAAD repaired patients requiring reintervention versus patients requiring no reintervention.

Chapter 2 highlights the importance of paving the road towards a better long-term outcome at the time of the index procedure for TAAD. A comparison of a conventional repair and an extensive repair revealed no compelling differences, but rather exemplified the patient-specific considerations that should already be made at the index procedure. Examples of these considerations are the presence of connective tissue disorder, the age, the location of the entry-tear, the extent of the dissection into branching vessels and the available expertise of the center regarding various types of repairs.

The search for predictors for residual native aortic growth is very timely, as endovascular reinterventions are becoming more adaptive and applicable. Outcomes of TEVAR as an intervention for type $B$ aortic dissection (TBAD) improve when performed early rather than late, ${ }^{24}$ and this may apply to residual TAAD in the descending thoracic aorta after conventional repair as well. Moreover, minimal invasiveness is favorable in an often fragile patient population, recovering from primary aortic intervention. ${ }^{25} \mathrm{Common}$ clinical predictors for residual adverse aortic remodeling are poor blood pressure management and connective tissue disease. ${ }^{16,17,26}$ For more case-specific determination of the need for aortic reintervention, radiographic analysis is key. Popular radiographic predictors that have been coined are often diameter-based. ${ }^{16,17,26}$

Chapter 3 demonstrates that the concept of the conventional computed tomography angiography (CTA) analysis is challenged in the case of TAAD. While one-dimensional 
diameters can easily be interpreted, the morphology of a dissection is rarely circular. One-dimensional diameters tend to misinform the clinician on aortic dynamics, growth and the sizing of the circular synthetic graft. However, two-dimensional measurements do provide this information. This thesis supports the use of area measurement, as the intra- and interobserver agreement is higher than for diameter measurements. Moreover, the currently available measuring software is well adapted for delineating areas, making the clinical use easily applicable. Interestingly, three-dimensional measurement (i.e. volumetric measurement) did not provide better agreement than two-dimensional measurement (i.e. area measurement), potentially because of the current laborious and time-consuming process with most software packages to delineate and compute the aortic volume of interest. Previous studies have suggested applying volumetric analysis to follow the dissected aorta before and after TEVAR repair. ${ }^{27,28}$ Despite the poor outcomes of volumetric analysis reliability presented in chapter 3 , our study group still believes that volumetric analysis may have additional value in monitoring aneurysms, true lumen or false lumen growth. However, it is believed that the measurement is currently too sensitive to error in order to be carried out by human delineation, as we found that volumetric delineation was best represented when carried out manually for each two-dimensional plane, causing for cumulative human error. Ideally, deep learning techniques should be applied to assess volumetric changes. On the other hand, the additional value of volumetric measurement for planning in endovascular procedures may be limited, as frequently the most relevant parts for the stent graft deployment are the proximal and distal landing zones. These planning measurements in the landing zones do require the local area measurement of the therapist. This is easily translated to a circular shape reflective of the actual stent graft as the square root of the area divided by $\pi$, making the amount of oversizing reflective of the actual area in the landing zone.

In the final chapter of this first part, chapter 4, TEVAR in TAAD is analyzed in a systematic review. The motivation for this systematic review was two-fold. First, endovascular repair in the ascending thoracic aorta has not yet been marketed, but is increasingly occurring across the globe and it needs to be quantified and qualified. Second, no systematic review on TEVAR in TAAD dissection had been described to date. The overall results were surprisingly positive when compared to open surgery, when one would expect a higher complication rate in an experimental none-invasive setting in a majority of patients deemed unfit for open surgery. Although the pioneering teams carrying out these procedures are often well-equipped in endovascular therapy and/or in aortic surgery in the treated area, the actual application of stenting over a longer access distance, in a more angulated area with high stresses and many important branches should raise irrefutable challenges. Moreover, selection bias due to prohibitive exclusion from conventional therapy may present another barrier. It is suggested that these results 
could be the effect of a certain publication bias, or rather lack of scientific publications, considering the supposed gap between the clinical market for TEVAR in TAAD and the provided evidence. In conclusion, further future reporting is required to draw any conclusions on clinical outcomes and our study group strongly suggests low-key reporting of any off-label results on TEVAR in TAAD, as large cohorts can be challenging without marketed devices.

\section{Part III - Descending thoracic aortic assessment}

In the third part of this thesis, an array of descending thoracic aortic pathologies, such as aneurysm, type B aortic dissection (TBAD) and intramural hematoma (IMH) is assessed by using a combination of literature-, clinical reporting-, imaging- and computational analysis. Chapter 5 provides a single center 16-year clinical analysis of TEVAR, emphasizing a mid-term follow-up in the endovascular disease. As expected, traumatic patients had a very different follow-up course compared to aneurysms and non-traumatic dissections. They were younger and relatively healthier, had a longer hospital stay with a higher mortality, although not often aorta-related. It was suggested that these patients be followed up to three years annually, after which follow-up could be continued with an extensive interval or only when symptoms occur. Furthermore, the chapter revealed an acceptable mortality rate in the overall population, but emphasized a long-term reintervention rate of more than $17.3 \%$, which was related to disease progression of the proximal and distal native aorta, rather than TEVAR related, in approximately half of the cases. The amount of long-term follow-up on TEVAR is rising. Only a handful of publications describe large center cohorts of all-disease TEVAR outcomes with an average follow-up over 4 years. ${ }^{29-31}$ These reports confirm a long-term reintervention rate of approximately $20 \%$. The need for long-term results is imperative and a good followup protocol is required, which incorporates inter-center infrastructure of clinical and radiographical material to adequately survey patients. This is the only way the lack of follow-up reporting in current literature due to centralization of care can be overcome.

TBAD is the most common acute descending aortic pathology. ${ }^{32}$ Intramural hematoma (IMH) is the second most common and shows many similarities with TBAD, such as a comparable initial clinical presentation. However, the disease entity is different and it may be challenging to target the correct landing zones and total stent graft zone in a circular disruption of the intimal layer, with no clear depiction of an entry-tear. These and other challenges are outlined in chapter $\mathbf{6}$, providing a comprehensive status of TEVAR in descending aortic IMH. It is remarkable to see that a limited amount of cohort studies on this specific disease entity had been published, with the largest study describing 66 patients. ${ }^{33}$ Although the current guidelines for thoracic aortic disease management clearly list TBAD as a separate disease requiring separate management from IMH, the 
clinical interpretation of the two seem to be intertwined and easily mixed up. Chapter 6 concluded that further large prospective clinical trials specifically focusing on IMH are required to better understand the natural history and to provide a stratified risk model. However, it might be more interesting to set up a multicenter blind exchange investigation, where incoming acute descending thoracic diseases are separately classified and screened for blind advice on diagnosis and operative procedure by an independent physician on paper. These advices will not be shared with the treating on-site physician and can later be compared to the actual main diagnosis and treatment that the patient received. This might help to further improve the knowledge on whether, clinically, the two entities are indeed considered different.

The $\mathbf{7}^{\text {th }}$ chapter on TEVAR in compromised distal landing zones, reveales how poor distal sealing has a significant effect on the reintervention rate and in term, the outcomes of TEVAR treated patients. The consequences of type Ib endoleak appear to be underreported compared to an overwhelming amount of prior type la reports. The inadequate reporting and potential underreporting of type $\mathrm{lb}$ endoleak was recently addressed by Belvroy et al. in a systematic review, including 16 studies. ${ }^{34}$ The group revealed a wide range of type $\mathrm{Ib}$ endoleak incidence from $1-15 \%$ and no available data regarding stent graft oversizing, length of distal landing zone or type of devices used. Chapter 7 of this thesis concludes that the use of adjuncts, being chimneys and/or EndoAnchors could potentially have a protective effect on the distal landing zone, providing more seal length. However, this was not significant and using the conventional method, procedural and fluoroscopy time was significantly lower $(p<0.005)$. Moreover, the conventional method using a tube graft without adjuncts is technically less challenging and could be more easily applied by many physicians. Ideally, branched and fenestrated custommade alternatives could limit the formation of gutters even more, potentially providing the best alternative for compromised distal landing zones. Additionally, a branched or fenestrated device rather than EndoAnchors or chimneys could leave a better option for landing during reintervention for extended pathology. However, branched devices are commonly designed for thoracoabdominal pathology, whereas the patients presented in this chapter were patients with primarily suprarenal pathology. A branched device with a single celiac trunk branch or double celiac trunk and superior mesenteric artery branch is not available, since the only way to acquire sufficient sealing zone length in pathology extending to this area immediately involves stenting the renal arteries. Additionally, through-and-through wiring for single branched devices, such as performed in the LSA is not possible in the visceral arteries. The alternative of using a full thoracoabdominal device with additional fenestrations or branches to the renal arteries poses unnecessary jeopardy for renal impairment, but moreover, poses an extra risk for spinal cord ischemia. Hence, supraceliac compromised distal landing zones pose a large 
challenge still in the current TEVAR age and was met with a high type Ib endoleak rate (35\%), which would definitely require more attention.

TBAD management outcomes have extensively been studied. Unfortunately, we are lacking an adequate predicting factor for dissection development. It is clear that high pressure and high pulse pressure are contributing factors to the incidence of dissection, ${ }^{35}$ potentially explaining why cocaine use is a risk factor for dissection. ${ }^{36}$ Likewise, connective tissue disease is a common risk factor, ${ }^{37}$ but does not inform on the progression of disease. The cardiovascular community turns to maximal aortic diameters, but the majority of dissecting patients has a maximal aortic diameter below standard surgical repair thresholds $(5.5 \mathrm{~cm}) .{ }^{38,39}$ Moreover, these studies are often based on postdissection diameters, which poorly reflects the pre-dissection diameter. Ultimately, chapter 3 further highlighted the limitations in diameter assessments. As maximal diameter currently is a flawed estimator for dissection or rupture risk, but still the best available, it is important that we find other parameters to non-invasively assess pending aortic acute pathology. It is believed that dissection is initiated by a local pattern of progressive loss of aortic wall integrity caused by medial degeneration, also resulting in aortic growth. A concept that has been termed as mechanobiological instability. ${ }^{40}$ This progression is difficult to catch by a manual diameter aortic measurement between CTA scans over time. In chapter $\mathbf{8}$ of this thesis, the correlation between the location of focal growth (i.e. local volumetric growth, such as bulging of an aorta at a specific site, which cannot be fully captured by diameter- or area-measurement), high cyclic transmural wall stress and entry-tear formation was investigated. A technique called vascular deformation mapping (VDM) was applied, to assess focal areas of growth in a three-dimensional overlay between two CT scans. Furthermore, a stress analysis in a computational aortic wall model was used to analyze cyclic transmural wall stress. All patients had two CTA scans before a TBAD occurred, allowing a focal growth analysis. Interestingly, all areas of focal growth and transmural stress co-localized with the site of the entry-tear formation. This two-pronged approach can allow for a sophisticated morphologic and biomechanical assessment that may improve our current understanding of the pathogenesis of aortic dissection.

\section{Part IV - Hemodynamic impact of graft repair}

Synthetic graft materials most often used for aortic repair are either polyethylene terephthalate (PET) or expanded polytetrafluoroethylene (PTFE). In the case of stent grafts for endovascular repair any of these materials cover the inside or outside of a nitinol or stainless steel stent. These synthetic polymers are currently up to 15 times as stiff as the native thoracic aorta. ${ }^{41-44}$ The stiffness mismatch has been investigated in ex-vivo settings, revealing an increase in overall stiffness after implantation of a stent 
graft in porcine models. This leads to increased longitudinal and radial aortic strain ${ }^{45-47}$ and increased pulse wave velocity and pulse pressure, especially in the proximal nonrepaired aorta. ${ }^{48,49} \mathrm{~A}$ recent computational fluid dynamic (CFD) analysis of eight patients treated with TEVAR was published by van Bakel et al. ${ }^{50}$ The left ventricular (LV) stroke work was estimated via patient-specific fluid structure interaction analyses, computing a $26 \%$ increase in LV stroke work post-TEVAR. CTA-volume measurements over a mean follow-up of 1.3 years generated a $15 \%$ increase in LV myocardial mass. The latter suggested that the increased stiffness mismatch introduced in the aorta, can be assessed morphologically in the LV due to adverse remodeling. In the light of these findings, a larger retrospective cohort analysis was performed to test the hypothesis. In chapter 9 of this thesis, a systematic short-term LV analysis of ECG-gated scans is described, through a refined selection process of patients undergoing ascending and/or root dilation repair, without having any other comorbidities or interventions that could severely influence LV remodeling, such as aortic valve replacement. The hypothesis was confirmed in a multivariable analyses in a surgically and in a medically treated group with proximal aortic dilation. A significant $7 \%$ increase in LV mass in 47 surgically repaired patients was observed within a mean 9.1 month follow-up. This was compared to a $1 \%$ increase in LV mass in 51 control-patients within a mean 13.7 month follow-up, receiving optimal medical therapy only $(p<0.001)$.

\section{Future perspective}

Early and mid-term results in TEVAR are superior to open repair in descending thoracic aortic pathology. Slowly emerging long-term results are promising. In ascending and arch TEVAR, early results are equally promising, although technical challenges and higher complication rate than the TEVAR in the descending aorta still exist, as outlined in this thesis.

The basic keys to future success are 1) obtaining the data in the most accurate and reproducible fashion and subsequently 2 ) objectifying these changes in a systematic fashion.

Obtaining the data in a future endovascular era should consist of three features. First, ECG-gated CTA has become a widely spread method for aortic analysis in aortic centers throughout the world. Its high resolution and adequately timed images, provide the most accurate anatomical information. However, nowadays limited hemodynamic information is gathered. As this thesis mostly focused on the morphologic assessment and outcomes of aortic interventions, anatomy is merely one factor of the full scope of endovascular analysis. Future scientific work on hemodynamic aortic assessment should be enriched by 4D-MRI to quantify flow and to be able to make temporary observations, as 
it outcompetes other non-invasive flow-measurement methods such as doppler echo of the aorta. Next to anatomic data and flow data, the third missing piece of the puzzle in order to adequately assess hemodynamics is pressure. Despite past developments such as catheter-based pressure measurements, applied tonometry or arterial cannulation, the most robust and applicable measuring pressure is still performed through brachial cuff pressures. Unfortunately, in clinical practice cuff pressures are not measured in a protocolled fashion at the time of imaging, set aside from the connotation that cuff pressures do not perfectly reflect the central aortic pressure. ${ }^{51}$ Its application could be the easiest quick fix, in order to improve clinical and scientific approach to aortic disease, as most of the investigations in aortic disease are performed retrospectively.

Next, a systematic fashion is required to quantify and qualify the data, as we process it in our analysis. Chapter 3 demonstrated how diameter measurements may be mistakenly applied to all aortic diagnosis and management situations, whereas the area could be more reliable. CFD, is now applied in a scientific field only, as real-time CFD is still too iterative, operator dependent and time-consuming. However, it might shortly prove useful in practice, providing non-invasive information on hemodynamics in complex anatomy and providing high resolution information for surgical planning and evaluation of surgical intervention and devices. It is important to have an automated workflow with clear systematic and community-wide uniform definitions in place. In expectancy of deep learning to perform segmentations of the vasculature in the near future, we should start adapting to limit flaws. This requires a continued integrated participation in health science by companies, engineers and physicians, taking translational medicine to a new level. Such a collaboration may also improve graft or interventional adjuncts design. Although a certain increased hemodynamic and biomechanical effect induced by aortic intervention seems inevitable, the stiffness mismatch with current material warrants the search for more conformable devices. The potential developments could improve interventional outcomes, which is currently needed, as could be seen in chapter 7 and 9 .

Finally, there is still a lack of reporting and a lack of long-term results. ${ }^{52}$ These need addressing and a major issue that should be dealt with is the poor follow-up as a result of centralization of specialized health care. Patients living in rural areas or with poor social-economic status lack the means to be adequately followed in nearby rural centers. Moreover, the required diagnostic and therapeutic advancements at these rural centers should also be monitored. Both these factors may contribute to the loss of quality data. In order to provide tools, to control and improve the quality of data being gathered at satellite centers, designated local data managers are required. They should not only follow the protocol, but contribute to the protocol development and the infrastructure for 
data-exchange. This will create a sense of involvement, which will in turn enhance the group process, increasing everyone's interest and effort in the joint goals. 


\section{REFERENCES}

1. Hagan PG, Nienaber CA, Isselbacher EM, et al. The International Registry of Acute Aortic Dissection (IRAD): new insights into an old disease. JAMA. 2000;283(7):897-903.

2. Conway BD, Stamou SC, Kouchoukos NT, et al. Improved clinical outcomes and survival following repair of acute type A aortic dissection in the current era. Interact Cardiovasc Thorac Surg. 2014;19(6):971-976. doi:10.1093/ icvts/ivu268

3. Pape LA, Awais M, Woznicki EM, et al. Presentation, diagnosis, and outcomes of acute aortic dissection: 17year trends from the international registry of acute aortic dissection. J Am Coll Cardiol. 2015;66(4):350-358. doi:10.1016/j.jacc.2015.05.029

4. Yang B, Rosati CM, Norton EL, et al. Endovascular Fenestration/Stenting First Followed by Delayed Open Aortic Repair for Acute Type A Aortic Dissection with Malperfusion Syndrome. Circulation. 2018;138(19):2091-2103. doi:10.1161/CIRCULATIONAHA.118.036328

5. Wang GJ, Jackson BM, Foley PJ, et al. National trends in admissions, repair, and mortality for thoracic aortic aneurysm and type B dissection in the National Inpatient Sample. J Vasc Surg. 2018;67(6):1649-1658. doi:10.1016/j.jvs.2017.09.050

6. Semba CP, Mitchell RS, Miller DC, et al. Thoracic aortic aneurysm repair with endovascular stent-grafts. VasC Med. 1997;2(2):98-103. doi:10.1177/1358863X9700200205

7. Patel HJ, Shillingford MS, Mihalik S, Proctor MC, Deeb GM. Resection of the Descending Thoracic Aorta: Outcomes After Use of Hypothermic Circulatory Arrest. Ann Thorac Surg. 2006;82(1):90-96. doi:10.1016/j.athoracsur.2006.02.055

8. Chiu P, Goldstone AB, Schaffer JM, et al. Endovascular Versus Open Repair of Intact Descending Thoracic Aortic Aneurysms. J Am Coll Cardiol. 2019;73(6):643-651. doi:10.1016/j.jacc.2018.10.086

9. Riambau V, Böckler D, Brunkwall J, et al. Management of Descending Thoracic Aorta Diseases: Clinical Practice Guidelines of the European Society for Vascular Surgery (ESVS). Eur J Vasc Endovasc Surg. 2017;53(1):4-52. doi:10.1016/j.ejvs.2016.06.005

10. Erbel R, Aboyans V, Boileau C, et al. 2014 ESC guidelines on the diagnosis and treatment of aortic diseases. RusS J Cardiol. 2015;123(7):7-72. doi:10.15829/1560-4071-2015-07-7-72

11. Hiratzka LF, Bakris GL, Beckman JA, et al. 2010 ACCF/AHA/AATS/ACR/ASA/SCA/SCAI/SIR/STS/SVM Guidelines for the Diagnosis and Management of Patients with Thoracic Aortic Disease. Anesth Analg. 2010;111(2):279-315. doi:10.1016/j.jacc.2010.02.010

12. Abraha I, Romagnoli C, Montedori A, Cirocchi R. Thoracic stent graft versus surgery for thoracic aneurysm. Cochrane Database Syst Rev. 2016;2016(6). doi:10.1002/14651858.CD006796.pub4

13. Andersen ND, Ganapathi AM, Hanna JM, Williams JB, Gaca JG, Hughes GC. Outcomes of acute type A dissection repair before and after implementation of a multidisciplinary thoracic aortic surgery program. J Am Coll Cardiol. 2014;63(17):1796-1803. doi:10.1016/j.jacc.2013.10.085

14. Bashir M, Harky A, Fok M, et al. Acute type A aortic dissection in the United Kingdom: Surgeon volume-outcome relation. J Thorac Cardiovasc Surg. 2017;154(2):398-406.e1. doi:10.1016/j.jtcvs.2017.02.015

15. Smith HN, Boodhwani M, Ouzounian M, et al. Classification and outcomes of extended arch repair for acute Type A aortic dissection: A systematic review and meta-analysis. Interact Cardiovasc Thorac Surg. 2017;24(3):450459. doi:10.1093/icvts/ivw355

16. Halstead JC, Meier M, Etz C, et al. The fate of the distal aorta after repair of acute type A aortic dissection. J Thorac Cardiovasc Surg. 2007;133(1):127-135. doi:10.1016/j.jtcvs.2006.07.043

17. Leontyev S, Haag F, Davierwala PM, et al. Postoperative Changes in the Distal Residual Aorta after Surgery for Acute Type A Aortic Dissection: Impact of False Lumen Patency and Size of Descending Aorta. Thorac Cardiovasc Surg. 2017;65(2):90-98. doi:10.1055/s-0036-1571813

18. Bojko MM, Suhail M, Bavaria JE, et al. Midterm outcomes of emergency surgery for acute type A aortic dissection in octogenarians. In: Journal of Thoracic and Cardiovascular Surgery. Vol 0. Mosby Inc.; 2020. doi:10.1016/j. jtcvs.2020.03.157 
19. Jussli-Melchers J, Panholzer B, Friedrich C, et al. Long-term outcome and quality of life following emergency surgery for acute aortic dissection type A: A comparison between young and elderly adults. Eur J Cardio-thoracic Surg. 2017;51(3):465-471. doi:10.1093/ejcts/ezw408

20. Tang GHL, Malekan R, Yu CJ, Kai M, Lansman SL, Spielvogel D. Surgery for acute type A aortic dissection in octogenarians is justified. J Thorac Cardiovasc Surg. 2013;145(3 SUPPL.):S186-S190. doi:10.1016/j.jtcvs.2012.11.060

21. Trimarchi S, Eagle KA, Nienaber CA, et al. Role of age in acute type A aortic dissection outcome: Report from the International Registry of Acute Aortic Dissection (IRAD). J Thorac Cardiovasc Surg. 2010;140:784-789. doi:10.1016/j.jtcvs.2009.11.014

22. Santini F, Montalbano G, Messina A, et al. Survival and quality of life after repair of acute type A aortic dissection in patients aged 75 years and older justify intervention. Eur J Cardio-thoracic Surg. 2006;29(3):386-391. doi:10.1016/j.ejcts.2005.12.016

23. Aoyama T, Kunisawa S, Fushimi K, Sawa T, Imanaka Y. Comparison of surgical and conservative treatment outcomes for type a aortic dissection in elderly patients. J Cardiothorac Surg. 2018;13(1):1-8. doi:10.1186/ s13019-018-0814-6

24. Heijmen R, Fattori R, Thompson M, et al. Mid-term outcomes and aortic remodelling after thoracic endovascular repair for acute, subacute, and chronic aortic dissection: The VIRTUE Registry. Eur J Vasc Endovasc Surg. 2014;48(4):363-371. doi:10.1016/j.ejvs.2014.05.007

25. Patel HJ, Williams DM, Upchurch GR, et al. A Comparison of Open and Endovascular Descending Thoracic Aortic Repair in Patients Older Than 75 Years of Age. Ann Thorac Surg. 2008;85(5):1597-1604. doi:10.1016/j.athoracsur.2008.01.044

26. Kim JB, Lee CH, Lee TY, et al. Descending aortic aneurysmal changes following surgery for acute DeBakey type I aortic dissection. Eur J Cardio-thoracic Surg. 2012;42(5):851-857. doi:10.1093/ejcts/ezs157

27. Stanley GA, Murphy EH, Knowles M, et al. Volumetric analysis of type B aortic dissections treated with thoracic endovascular aortic repair. J Vasc Surg. 2011;54(4):985-992. doi:10.1016/j.jvs.2011.03.263

28. Tolenaar JL, Kern JA, Jonker FH, et al. Predictors of false lumen thrombosis in type B aortic dissection treated with TEVAR. Ann Cardiothorac Surg. 2014;3(3):255-263. doi:10.3978/j.issn.2225-319X.2014.05.17

29. Patel HJ, Williams DM, Drews JD, et al. A 20-year experience with thoracic endovascular aortic repair. Ann Surg. 2014;260(4):691-697. doi:10.1097/SLA.0000000000000930

30. Ziza V, Canaud L, Molinari N, Branchereau P, Marty-Ané C, Alric P. Thoracic endovascular aortic repair: A single center's 15-year experience. J Thorac Cardiovasc Surg. 2016;151(6):1595-1603.e7. doi:10.1016/j. jtcvs.2015.12.030

31. Wiedemann D, Mahr S, Vadehra A, et al. Thoracic endovascular aortic repair in 300 patients: Long-term results. Ann Thorac Surg. 2013;95(5):1577-1583. doi:10.1016/j.athoracsur.2013.02.043

32. DeMartino RR, Sen I, Huang Y, et al. Population-Based Assessment of the Incidence of Aortic Dissection, Intramural Hematoma, and Penetrating Ulcer, and Its Associated Mortality From 1995 to 2015. Circ Cardiovasc Qual Outcomes. 2018;11(8):e004689. doi:10.1161/CIRCOUTCOMES.118.004689

33. Von Kodolitsch Y, Csösz SK, Koschyk DH, et al. Intramural Hematoma of the Aorta Predictors of Progression to Dissection and Rupture. Circulation. 2003;107:1158-1163. doi:10.1161/01.CIR.0000052628.77047.EA

34. Belvroy VM, de Beaufort HWL, van Herwaarden JA, Trimarchi S, Moll FL, Bismuth J. Type 1b Endoleaks After Thoracic Endovascular Aortic Repair are Inadequately Reported: A Systematic Review. Ann Vasc Surg. 2019. doi:10.1016/j.avsg.2019.06.030

35. Baguet JP, Chavanon O, Sessa C, et al. European Society of Hypertension scientific newsletter: Hypertension and aortic diseases. J Hypertens. 2012;30(2):440-443. doi:10.1097/HJH.0B013E32834F867A

36. Cregler L. Aortic dissection and cocaine use. Am Heart J. 1992;124(6):1665. doi:10.1016/0002-8703(92)90109-9

37. Nienaber CA, Clough RE. Management of acute aortic dissection. Lancet. 2015;385(9970):800-811. doi:10.1016/ S0140-6736(14)61005-9

38. Trimarchi S, Jonker FHW, Froehlich JB, et al. Acute type B aortic dissection in the absence of aortic dilatation. J Vasc Surg. 2012;56(2):311-316. doi:10.1016/j.jvs.2012.01.055

39. Berezowski M, Kosiorowska K, Beyersdorf F, et al. Modelling of predissection aortic size in acute descending aortic dissection. Interact Cardiovasc Thorac Surg. 2019;29(1):124-129. doi:10.1093/icvts/ivz028 
Discussion

40. Cyron CJ, Wilson JS, Humphrey JD. Mechanobiological stability: A new paradigm to understand the enlargement of aneurysms? J R Soc Interface. 2014;11(100). doi:10.1098/rsif.2014.0680

41. Spadaccio C, Nappi F, Al-Attar N, et al. Old Myths, New Concerns: the Long-Term Effects of Ascending Aorta Replacement with Dacron Grafts. Not All That Glitters Is Gold. J Cardiovasc Transl Res. 2016;9(4):334-342. doi:10.1007/s12265-016-9699-8

42. Ioannou C V., Stergiopulos N, Katsamouris AN, et al. Hemodynamics induced after acute reduction of proximal thoracic aorta compliance. Eur J Vasc Endovasc Surg. 2003;26(2):195-204. doi:10.1053/ejvs.2002.1917

43. Roccabianca S, Figueroa CA, Tellides G, Humphrey JD. Quantification of regional differences in aortic stiffness in the aging human. J Mech Behav Biomed Mater. 2014;29:618-634. doi:10.1016/j.jmbbm.2013.01.026

44. Tremblay D, Zigras T, Cartier R, et al. A Comparison of Mechanical Properties of Materials Used in Aortic Arch Reconstruction. Ann Thorac Surg. 2009;88(5):1484-1491. doi:10.1016/j.athoracsur.2009.07.023

45. Nauta FJH, Conti M, Marconi S, et al. An experimental investigation of the impact of thoracic endovascular aortic repair on longitudinal strain. Eur J Cardio-thoracic Surg. 2016;50(5):955-961. doi:10.1093/ejcts/ezw180

46. Nauta FJH, de Beaufort HWL, Conti M, et al. Impact of thoracic endovascular aortic repair on radial strain in an ex vivo porcine model. Eur J Cardio-thoracic Surg. 2017;51(4):783-789. doi:10.1093/ejcts/ezw393

47. Nauta FJH, Van Bogerijen GHW, Trentin C, et al. Impact of thoracic endovascular aortic repair on pulsatile circumferential and longitudinal strain in patients with aneurysm. J Endovasc Ther. 2017;24(2):281-289. doi:10.1177/1526602816687086

48. De Beaufort HWL, Coda M, Conti M, et al. Changes in aortic pulse wave velocity of four thoracic aortic stent grafts in an ex vivo porcine model. PLoS One. 2017;12(10). doi:10.1371/journal.pone.0186080

49. de Beaufort HWL, Conti M, Kamman A V., et al. Stent-Graft Deployment Increases Aortic Stiffness in an Ex Vivo Porcine Model. Ann Vasc Surg. 2017;43:302-308. doi:10.1016/j.avsg.2017.04.024

50. van Bakel TM, Arthurs CJ, Nauta FJ, et al. Cardiac remodeling following thoracic endovascular aortic repair for descending aortic aneurysms. Eur J Cardio-Thoracic Surg. 2018;0:1-10. doi:10.1016/0038-1098(79)91043-3

51. Agabiti-Rosei E, Mancia G, O'Rourke MF, et al. Central blood pressure measurements and antihypertensive therapy: A consensus document. Hypertension. 2007;50(1):154-160. doi:10.1161/HYPERTENSIONAHA.107.090068

52. Kret MR, Azarbal AF, Mitchell EL, Liem TK, Landry GJ. From the Western Vascular Society Compliance with longterm surveillance recommendations following endovascular aneurysm repair or type B aortic dissection. J Vasc Surg. 2013;58:25-32. doi:10.1016/j.jvs.2012.12.046 


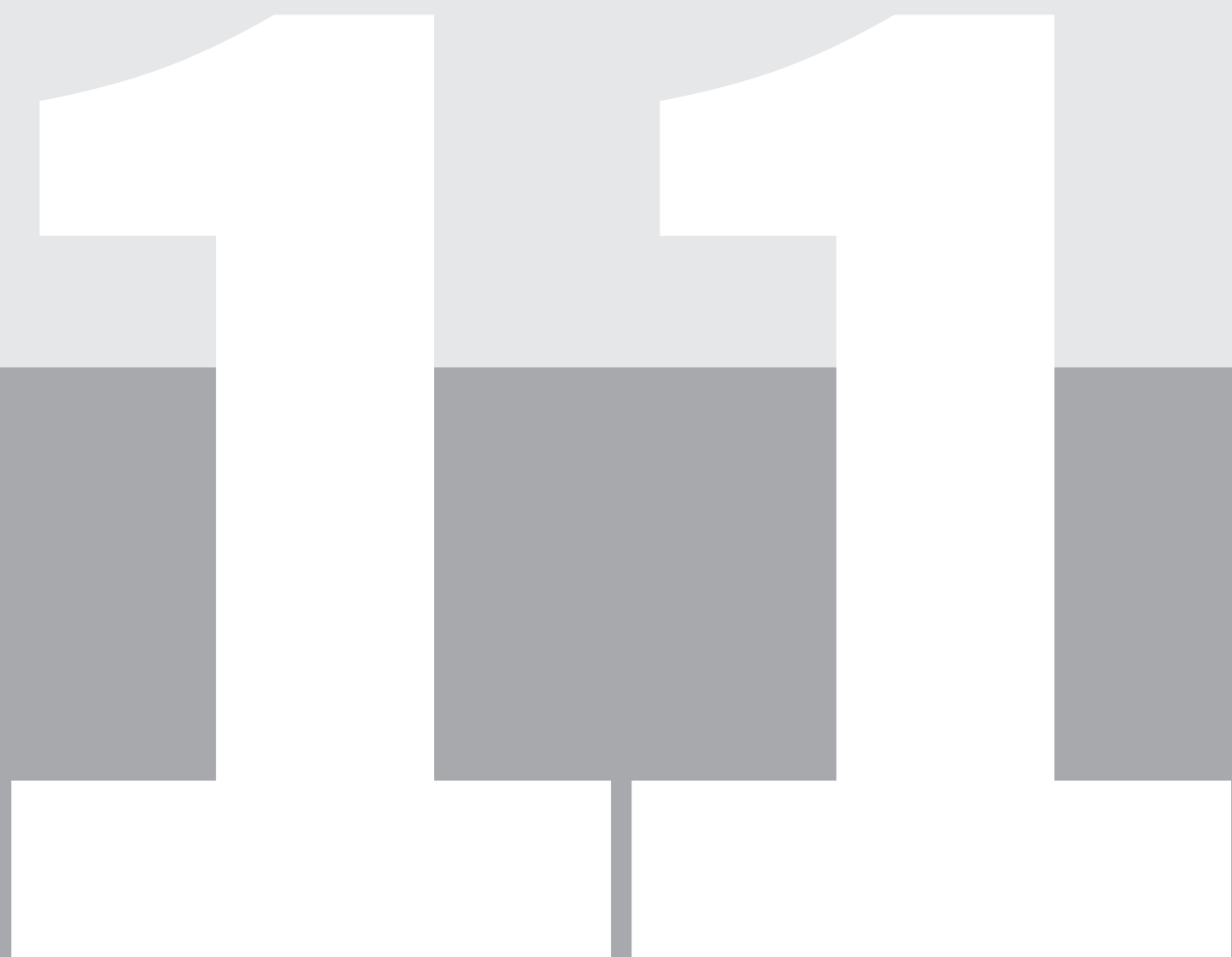
Discussie in het Nederlands 



\section{DISCUSSIE IN HET NEDERLANDS}

De beoordeling en behandeling van thoracale aorta pathologie heeft de laatste 30 jaar een enorme ontwikkeling doorgemaakt. De korte ( $\leq 6$ maanden) en middellange termijn ( $\leq 5$ jaar) mortaliteit voor zowel aneurysmata als dissecties is aanzienlijk verminderd. Voor dissecties geldt een daling $26-31 \%$ naar circa $10-18 \% .^{1-5}$ Voor aneurysmata is deze daling van 10-45\% naar 5-25\%, waarbij de grote bandbreedte is toe te schrijven aan verschillen in uitkomsten afhankelijk van hoe "acuut", ofwel symptomatisch het aneurysma zich presenteert. ${ }^{5-9}$ Risicofactoren zijn dusdanig onderzocht dat in toenemende mate op patiënt-specifiek niveau een risicoprofiel kan worden ingeschat, met de juiste begeleidende behandeling. Thoracale endovasculaire aorta repair (TEVAR) is de meest toegepaste niet-medicamenteuze interventie voor thoracale aorta pathologie in de aorta descendens. ${ }^{9-11}$ Echter, tot op heden werden er nog geen gerandomiseerde klinische studies uitgevoerd om de oude gouden standaard (buisprothese middels open chirurgie) te vergelijken met de nieuwe gouden standaard (TEVAR). ${ }^{12}$ De internationale richtlijnen dragen level 2 en 3 bewijs aan om de keuze voor TEVAR boven open chirurgie te legitimeren. ${ }^{9-11}$ De hoeveelheid studies met relatief goede korte termijn uitkomsten van TEVAR is weliswaar zeer overtuigend. Dit is een bewijs dat snelle translationele technische ontwikkelingen in endovasculaire chirurgie zo snel gaan dat gerandomiseerde onderzoeken - welke van oudsher als de gouden standaard worden gezien - overbodig lijken. Bovendien zijn de eerste patiënten die regelmatig geïncludeerd worden voor nieuwe minder invasieve methoden niet in staat om open chirurgie te ondergaan, wat randomisatie in de weg staat. Gezien de snelheid van deze ontwikkelingen is het van vitaal belang om in de gaten te houden wat de lange termijn effecten zijn van TEVAR. Blijft de behandeling met een vergrijzende populatie superieur aan open chirurgie? En hoe kunnen we deze lange termijn effecten en de pre-existente risico factoren voor eventueel falen van de behandeling beter in kaart brengen?

Dit proefschrift heeft als doel om een bijdrage te leveren aan het begrijpen van de beeldvorming rond endovasculaire therapie en de hemodynamische gevolgen van endovasculaire therapie, alsmede het in kaart brengen van recent klinisch onderzoek. $\mathrm{Na}$ een introductie in deel I wordt in deel II gestart met de beoordeling van conventionele versus progressieve therapie in type $A$ aorta dissecties (TAAD), gericht op de complete behandeling van de volledige aorta, in plaats van een solitaire focus op de intimascheur. Verder wordt de weg naar minimaal-invasieve chirurgie in het laatste domein van open aorta chirurgie - de aorta ascendens - verkend. In deel III worden de meest recente uitdagingen in TEVAR van de aorta descendens geëvalueerd. Verder wordt een multiparametrische methode onderzocht om een biomechanische conceptuele evaluatie van aneurysma en dissectie formatie te verrichten. Tenslotte, in deel IV, worden de 
theoretische hemodynamische veranderingen ten gevolge van de introductie van stijf synthetisch materiaal in de aorta geëvalueerd met behulp van morfologische metingen.

\section{Deel II - Beoordeling en behandeling van type A aorta dissecties}

TAAD blijft een van de weinige aorta pathologieën waar reparatie middels open chirurgie de onbetwiste gouden standaard is. ${ }^{9-11}$ Deze ingreep vereist tijdige en gespecialiseerde interventie, waarbij zichtbaar is dat de vroege overleving aanzienlijk verbeterde nadat toegewijde aortachirurgie teams met concentratie in gespecialiseerde aorta verwijscentra zich hebben ontwikkeld. ${ }^{13,14}$ De huidige 30-dagen postoperatieve overleving is circa $80-85 \%$ voor alle patiënten die levend het ziekenhuis bereiken en fit genoeg zijn om geopereerd te worden., ${ }^{3,4,15}$ Hoewel er waarschijnlijk altijd ruimte zal zijn voor acute technische verbetering, zou er aandacht moeten zijn voor de ontwikkeling van een model voor lange termijn predictie. Ondanks goede overlevingscijfers, laten follow-up studies zien dat 1-2 op de 10 overlevende patiënten binnen circa 10 jaar een re-operatie ondergaan ter voorkoming van ruptuur van de resterende aortadissectie (i.e. de resterende natieve aorta, inclusief het ware en valse lumen dat blijft bestaan na initiële chirurgische interventie ter plaatse van de intimascheur). ${ }^{16,17}$ Wanneer we beter inzicht krijgen in het risicoprofiel van re-operatie behoevende patiënten, kunnen we niet alleen een meer op maat gemaakte initiële behandeling bieden, maar verbeteren we ook de levenskwaliteit. Er zijn retrospectieve levenskwaliteit analyses na TAAD uitgevoerd. Aangezien bekend is dat leeftijd een significante risico factor is voor hogere mortaliteit na TAAD, zou intuïtief de verwachting zijn dat leeftijd ook een rol speelt in levenskwaliteit na TAAD. Echter, multipele studies laten zien dat levenskwaliteit in verschillende leeftijdsgroepen hetzelfde is na TAAD operatie. ${ }^{18-20}$ Voor de initiële operatie geldt dat tachtigjarigen een survival benefit lijken te verliezen bij operatief versus conservatief behandelen binnen 3 tot 5 jaar na diagnose stelling. ${ }^{21-23}$ Deze mortaliteitscijfers zouden wellicht als minder relevant kunnen worden beschouwd, in vergelijking met de levenskwaliteit, bedenkend dat de gemiddelde levensverwachting in vrijwel alle landen lager ligt dan 83 jaar. Re-operatie cijfers zouden dan een prominentere rol kunnen hebben in de beoordeling van levenskwaliteit, gezien het feit dat iedere re-sternotomie gepaard gaat met aanzienlijke revalidatie periodes. Tot op heden heeft geen enkele studie de levenskwaliteit beoordeeld van TAAD patiënten die een re-operatie kregen, versus patienten die geen re-operatie ondergingen.

Hoofdstuk 2 benadrukt het belang van een bewuste aandacht voor betere lange termijn uitkomsten en preventie van re-operatie, reeds tijdens de initiële index procedure. Een vergelijking van conventioneel herstel en uitgebreid herstel van de aorta liet geen grote verschillen zien, maar liet zien dat overwegingen ten aanzien van patiënt-specifieke factoren gemaakt moeten worden tijdens de index procedure. Deze factoren zijn bij- 
voorbeeld bindweefselaandoeningen, leeftijd, de locatie van de intimascheur, het verloop van de dissectie naar de aftakkende arteriën of de beschikbare expertise van het centrum met betrekking tot verschillende type operaties.

De zoektocht naar voorspellers van groei van de resterende aortadissectie is relevant, omdat endovasculaire re-operaties adaptiever en daarmee vaker toepasbaar zijn. De uitkomsten van TEVAR als interventie voor type B aorta dissecties (TBAD) zijn beter wanneer ze vroeg worden uitgevoerd. ${ }^{24}$ Dit geldt mogelijk ook voor resterende aortadissecties na conventioneel herstel. Bovendien heeft minimaal invasieve behandeling de voorkeur in een fragiele patiëntenpopulatie, welke herstellende is van de primaire aorta operatie. ${ }^{25}$ Bekende klinische voorspellers voor ongunstige remodellering van de oorspronkelijk aorta zijn slechte tensiecontrole en bindweefselaandoeningen. ${ }^{16,17,26}$ Voor de meer casus-specifieke inschatting van het re-operatie risico, speelt radiografische analyse een belangrijke rol. Populaire radiografische voorspellers zijn vaak gebaseerd op diameter metingen. ${ }^{16,17,26}$

Hoofdstuk 3 toont dat het concept van conventionele metingen middels computed tomography angiografie (CTA) niet voldoet in TAAD. Hoewel eendimensionale diameter metingen eenvoudig te interpreteren zijn, is de morfologie bij een dissectie zelden circulair. Eendimensionale diameters kunnen de clinicus misleiden ten aanzien van de aortadynamiek, de groei en de beoogde afmetingen van de stent graft. Tweedimensionale metingen, daarentegen, kunnen deze informatie wel geven. Dit proefschrift ondersteunt het gebruik van oppervlakte metingen boven die van eendimensionale metingen, gezien de hogere intra- en interobserver overeenkomst. Bovendien is de huidige beschikbare software voor aortametingen goed in staat oppervlakte metingen te faciliteren, waardoor het ook in de kliniek bruikbaar is. Driedimensionale metingen bleken in dit onderzoek niet superieur ten opzichte van tweedimensionale metingen en lijken bovendien met de huidige software nog niet gebruiksvriendelijk voor de klinische setting. Voorgaande studies suggereerden de toepassing van volume analyse voor follow-up van de aortadissectie voor en na operatie. ${ }^{27,28}$ Ondanks de niet-superieure betrouwbaarheid en het tijdrovende karakter van driedimensionale analyse was onze onderzoeksgroep er nog steeds van overtuigd dat volume analyse toegevoegde waarde kan hebben in het meest nauwkeurig monitoren van een aneurysma of het ware en/of valse lumen in een dissectie. We denken echter dat volume analyse momenteel te onderhevig is aan menselijke cumulatieve foutmeting. Idealiter zouden deep learning technieken toegepast moeten worden om volume analyses uit te voeren. Voor de klinische setting van het plannen en plaatsen van de stent graft heeft een volledige volume meting vaak geen meerwaarde, omdat de landingszones proximaal en distaal vaak het meest van belang zijn. Voor die landingszones zouden lokale oppervlakte metingen bijvoorbeeld een betere optie zijn. 
Deze kunnen makkelijk vertaald worden naar bruikbare diameter metingen voor de stent graft afmetingen door $\sqrt{ }(\mathrm{A} / \pi)$, waarbij $A$ de oppervlakte in $\mathrm{mm}^{2}$ is. De diameter metingen die daaruit voortkomen zijn een betere maat voor de oversizing van de stent graft dan de maatvoering voortkomend uit een directe diameter meting.

Het laatste hoofdstuk van het eerste deel, hoofdstuk 4, bestaat uit een systematische review naar het gebruik van TEVAR als behandeling van TAAD. De motivatie voor deze systematische review was tweeledig. Enerzijds was het doel om het gebruik van TEVAR in TAAD te kwantificeren en kwalificeren, aangezien het wereldwijd steeds vaker voorkomt, terwijl er nog geen goedgekeurde stent graft voor de aorta ascendens op de markt is. Anderzijds was er nog geen systematische review verschenen over TEVAR bij TAAD. De algehele resultaten waren opvallend positief, in vergelijking met chirurgie, terwijl een hoger aantal complicaties zou worden verwacht in een experimentele setting met een meerderheid van patiënten die als niet fit genoeg werd gezien voor open chirurgie. Hoewel de pioniersteams die de operatie uitvoerden vaak veel endovasculaire expertise in huis hadden, werd verwacht dat er ontegenzeggelijke uitdagingen op de loer lagen, zoals een langer toegangstraject, meer angulatie, de hogere drukken in het ascendens systeem en belangrijke nabijgelegen aftakkende arteriën. Daarnaast kan de geïncludeerde patiëntenpopulatie die voornamelijk niet fit genoeg was voor open chirurgie ook voor een selectie bias zorgen. Er werd concluderend gesuggereerd dat de resultaten mogelijk onderhevig waren aan publicatie bias, door een gebrek aan grote vergelijkende cohortstudies, dan wel gerandomiseerde studies, wat ertoe kan leiden dat onderzoekers alleen ervaringen opschrijven bij goede resultaten. Derhalve wordt in dit hoofdstuk een pleidooi gehouden voor meer rapportage van off-label resultaten van het gebruik van TEVAR in TAAD, ook bij slechte uitkomsten.

\section{Deel III - Beoordeling en behandeling van de thoracale aorta descendens}

In het derde deel van het proefschrift, worden diverse vormen van aorta descendens pathologie behandeld, zoals aneurysmata, TBAD en intramurale hematomen (IMH). In hoofdstuk 5 werd een mono center cohort analyse gedaan naar 16 jaar ervaring met TEVAR in de aorta descendens, met een nadruk op middellange termijn follow-up data, waarbij de 30-dagen uitkomsten bewust buiten beschouwing worden gelaten. Zoals verwacht, hadden de patiënten met traumatisch aorta letsel een andere follow-up vergeleken met patiënten met bijvoorbeeld een aneurysma of dissectie. De patiënten met traumatisch letsel waren over het algemeen relatief jong en gezond en hadden een langer ziekenhuisverblijf door overige letsels, gepaard gaande met hoge mortaliteit, welke niet vaak aorta-gerelateerd was. Voorgesteld wordt om deze patiënten tot drie jaar na het ongeval jaarlijks te controleren, waarna het interval in overleg met de patiënt kan worden versoepeld, eventueel zelfs tot revisie enkel bij symptomen. Het hoofdstuk 
toont verder een acceptabele mortaliteit in de gehele populatie, maar toont een lange termijn re-operatie hoeveelheid van $17.3 \%$, welke in de helft van de gevallen gerelateerd was aan progressieve groei van de niet door stent bedekte oorspronkelijke aorta, in plaats van aan de stent graft zelf. De hoeveelheid lange termijn follow-up studies na TEVAR neemt toe. Slechts enkele publicaties beschrijven een cohort studie met uitkomsten van alle TEVARs met een gemiddelde follow-up van meer dan vier jaar. ${ }^{29-31}$ Deze studies bevestigen lange termijn re-operatie aantallen van circa $20 \%$. De noodzaak voor lange termijn resultaten is essentieel en een goed follow-up protocol met incorporatie van veilige infrastructuur tussen zorgcentra van klinische en radiografische data zou daar idealiter een onderdeel van zijn. Dat is de enige manier om het gebrek aan follow-up data per centrum op te lossen.

TBAD is de meest voorkomende acute pathologie in de aorta descendens. ${ }^{32} \mathrm{IMH}$ is daarna het meest voorkomend en toont veel vergelijkingen met TBAD, zoals vergelijkbare initiele klinische presentatie. Echter, de entiteit van de ziekte is verschillend en het kan lastig zijn om de goede landingszones te kiezen in een circulaire dissectie zonder duidelijke intimascheur. Deze en andere uitdagingen worden besproken in hoofdstuk $\mathbf{6}$, welke een volledig beeld probeert te geven van TEVAR in IMH in de aorta descendens. Het is opvallend hoe weinig cohort studies over deze entiteit zijn gepubliceerd. De grootste cohort studie beschrijft 66 patiënten met IMH. ${ }^{33}$ De richtlijnen onderschrijven het verschillende karakter van dissecties en IMH en beschrijven dat hier in de behandeling rekening mee moet worden gehouden. Toch lijkt de klinische interpretatie van beide diagnosen en de uiteindelijke behandeling erg aan elkaar verbonden. Derhalve zijn er meer grotere studies nodig naar IMH om het natuurlijke beloop van de ziekte nog beter te begrijpen en om een adequaat risicoprofiel te ontwikkelen. Het zou mogelijkerwijs interessant zijn om een geblindeerd uitwisselingsonderzoek te doen, waarbij beeldvorming van acute thoracale aorta descendens pathologie separaat geclassificeerd wordt op diagnose en behandeladvies door een onafhankelijke chirurg. Deze diagnoses en adviezen worden niet gedeeld met het behandelende centrum, maar de data kunnen uiteindelijk wel vergeleken worden met de toegepaste diagnose en behandeling. Hiermee kan meer inzicht verkregen worden in welke mate de twee entiteiten dissecties en intramurale hematomen verschillend worden benaderd.

Hoofdstuk 7 behandelt TEVAR in patiënten met een gecompromitteerde distale landingszone, waarbij wordt aangetoond dat matige sealing van de distale landingszone een significant effect heeft op de re-operatie aantallen en daarmee op de uitkomsten van TEVAR. Over de consequenties van type lb endoleak lijkt weinig gerapporteerd te worden in vergelijking met een overweldigende hoeveelheid studies naar type la endoleaks. Belvroy et al besteedden recent aandacht aan de lagere en inadequate rappor- 
tage van type Ib endoleaks in een systematische review met 16 geïncludeerde studies. ${ }^{34}$ Onder deze 16 studies werd een breed bereik van type Ib endoleak incidentie gezien van 1-15\%, zonder enige beschikbare data omtrent stent graft oversizing, lengte van distale landingszone of het gebruikte type stent graft. In hoofdstuk 7 wordt geconcludeerd dat het gebruik van adjuncten (ofwel hulpstukken of hulpprothesen) als schoorstenen en/ of EndoAnchors een beschermend effect zou kunnen hebben op de distale landingszone. Echter, dit effect was niet significant en zonder adjuncten, dat wil zeggen met een conventionele buisprothese was de procedurele tijd en de angiografie tijd significant lager $(p<0.005)$. Daar komt bij dat deze procedures waarschijnlijk technisch eenvoudiger zijn en dus laagdrempeliger als standaard procedure ingezet zouden kunnen worden. Idealiter zouden op maat gemaakte stent grafts met vaste aftakkingen of openingen voor aftakkende vaten gebruikt moeten worden, om de kans op formatie van een goot te beperken. Daarnaast wordt met een dergelijke stent graft met ingebouwde openingen of aftakkende stent grafts de landingzone vergroot om met meer zekerheid het gecompromitteerde deel te overbruggen. Helaas worden deze type grafts normaliter ontwikkeld voor thoracoabdominale pathologie, terwijl de patiënten in onze analyse hoofdzakelijk suprarenale pathologie hadden. Er bestaan geen stent grafts met een enkele aftakking voor de truncus coeliacus en de arteria mesenterica superior, aangezien de landingszone de arteriae renales zou overlappen. Verder is het door-en-door plaatsen van een guidewire - voor stent graft ontplooiing in lange geanguleerde trajecten - niet mogelijk bij de visceraal aftakkende vaten. Bovendien gaat een volledige thoracoabdominale stent graft plaatsen gepaard met mogelijke nierfunctie stoornissen of spinaal ischemie. Dit maakt een gecompromitteerde supracoeliacale distale landingszone een lastig fenomeen, gepaard gaand met een hoge hoeveelheid type lb endoleaks (35\%), waarvoor wat de onderzoekers betreft aandacht nodig is.

De uitkomsten van TBAD zijn wereldwijd uitgebreid bestudeerd. Helaas mist er nog een adequate voorspellende factor voor de ontwikkeling van dissecties. Hypertensie en een hoge polsdruk zijn bijdragende factoren voor de ontwikkeling van een dissectie, ${ }^{35}$ wat mogelijk verklaart waarom cocaïne gebruik een significante risico factor is voor het ontwikkelen van een aortadissectie. ${ }^{36}$ Zo is een bindweefselaandoening ook een bekende risicofactor, ${ }^{37}$ maar het geeft geen informatie over de progressie van ziekte en daarmee het moment in het leven van de patiënt waarop het risico groot genoeg is om een interventie te rechtvaardigen. De cardiovasculaire gemeenschap wendt zich tot maximale aorta diameters, maar de meerderheid van dissecties lijkt plaats te vinden onder de standaard afkapwaarde voor operatieve interventie $(5,5 \mathrm{~cm}) .^{38,39}$ Deze studies zijn vaak gebaseerd op post-dissectie diameters, welke een matige afspiegeling zijn van pre-dissectie diameters. Bovendien laat hoofdstuk 3 al zien wat de beperkingen zijn van diameter metingen in aorta dissecties. Aangezien de maximale diameter van de aorta 
een foutgevoelige voorspeller is voor het inschatten van een aortadissectie of -ruptuur, is het belangrijk dat er andere meetbare parameters worden gevonden, waarmee op non-invasieve wijze het dreigende ruptuur risico kan worden ingeschat. Er bestaat een concept dat beschrijft dat dissecties beginnen via een lokaal patroon met progressief verlies van aortawand integriteit middels tunica media degeneratie. Hierdoor ontstaat lokale groei ofwel uitstulping van de aortawand. Dit concept is gestoeld op mechanobiologische instabiliteit. ${ }^{40}$ Deze groei is echter zo minimaal dat het lastig te vangen is via manuele aorta diameter metingen tussen CTA scans. In hoofdstuk $\mathbf{8}$ wordt beschreven hoe de correlatie tussen de locatie van focale groei (i.e. het centrum van lokale uitstulping op een specifieke plek in de aortawand, welke niet volledig gevangen kan worden via diameter- of oppervlakte meting), hoge cyclische (per hartcyclus) transmurale aortawand stress en de formatie van een intimascheur werd onderzocht. Hiervoor werd een techniek gebruikt die vascular deformation mapping (VDM) wordt genoemd, om de focale groei te vinden middels een overlap techniek van twee CTA scans. Verder werd stress analyse gebruikt middels een computational dynamics aorta model - ofwel een rekenkundig computer model - om cyclische transmurale aortawand stress te berekenen. Van iedere patiënt werden twee CTA scans gebruikt voorafgaand aan TBAD, waardoor een focale groei analyse mogelijk was. Opvallend is dat alle gebieden van focale groei en hoge transmurale aortawand stress overeenkwamen met het gebied waar de intimascheur optrad. Deze tweeledige aanpak van focale groei metingen en stress metingen kan leiden tot meer geavanceerde morfologische en biomechanische analyse, waardoor ons begrip ten aanzien van de pathogenese van een aortadissectie verdiept kan worden.

\section{Deel IV - Hemodynamische impact van prothese vervanging van de aorta}

De synthetische materialen die meestal gebruikt worden voor aortaprothesen zijn polyethyleentereftalaat (PET) of geëxpandeerd polytetrafluoretheen (PTFE). In het geval van stent grafts voor een endovasculaire behandeling bekleden deze materialen vaak de binnenzijde van een nitinol stent. De synthetische polymeren zijn 15 maal stijver dan de natieve thoracale aorta. ${ }^{41-44}$ Deze stijfheid mismatch tussen het materiaal en de natieve aorta is onderzocht in ex-vivo opzet, waarbij de volledige verandering in stijfheid in het volledige aorta systeem van een varkensmodel werd geëvalueerd na implantatie van de stent graft. Implantatie leidde tot verhoogde longitudinale en radiale aorta deformatie ${ }^{45-47}$ en tot verhoogde polsgolfsnelheid en tensie, voornamelijk in het proximale, niet herstelde deel van de aorta. ${ }^{45-49}$ Recentelijk werden computational fluid dynamics gebruikt ter evaluatie van hemodynamische veranderingen binnen acht patiënten behandeld met TEVAR, gepubliceerd door van Bakel et al. ${ }^{50} \mathrm{Er}$ werd aangetoond dat de pompkracht die de linker ventrikel moet leveren naar schatting met $26 \%$ verhoogd werd na implantatie van de stent graft in de thoracale aorta. CTA-volume metingen over gemiddeld 1.3 jaar follow-up toonden bovendien een 15\% toename van linker ventrikel 
myocardium volume. Dit suggereerde dat de verhoogde stijfheid mismatch die door de stent graft in de aorta wordt geïntroduceerd, een nadelig remodellerings effect heeft, dat morfologisch kon worden beoordeeld in het linker ventrikel. Naar aanleiding van deze bevindingen, werd een grotere retrospectieve cohort analyse uitgevoerd om de bovenstaande hypothese te testen. In hoofdstuk 9 van dit proefschrift, wordt een systematische korte termijn CTA follow-up analyse beschreven. Hierbij werd gebruik gemaakt van electrocardiogram (ECG)-geleide scans, waarmee het volume van het linker ventrikel myocardium werd gemeten tussen twee verschillende follow-up scans. Het doel was om de mate van myocardiale remodellering te meten na protheseplaatsing in de aorta ascendens in verband met een verwijde aorta. Hierbij werd vergeleken met een groep patiënten die ook een aorta ascendens verwijding hadden maar slechts medicamenteus werden behandeld. Patiënten met een comorbiditeit of ingreep in het verleden die een grote invloed zouden hebben op linker ventrikel remodellering, zoals een aortaklepstenose of aortaklepvervanging werden geëxcludeerd. De hypothese werd bevestigd na multivariabele analyse. Een significante $7 \%$ linker ventrikel massa toename in 47 chirurgisch behandelde patiënten werd geobserveerd met een gemiddelde 9.1 maanden follow-up. De controle groep van 51 medicamenteus behandelde patiënten toonde slechts een $1 \%$ toename in linker ventrikel massa met een gemiddelde 13.7 maanden follow-up $(p<0.001)$.

\section{Toekomstperspectief}

Korte- en middellange termijn resultaten na TEVAR zijn superieur aan vergelijkbare resultaten na open chirurgie in thoracale aorta descendens pathologie. De eerste langetermijn resultaten zijn veelbelovend. In aorta ascendens en boog TEVAR, zijn resultaten ook veelbelovend, alhoewel technische uitdagingen blijven bestaan en de hoeveelheid complicaties nog hoger lijkt in vergelijking met TEVAR in de aorta descendens. De basisstappen voor succes in de toekomst van onderzoek naar aorta behandeling zijn 1) het verkrijgen van data op een accurate en reproduceerbare manier en vervolgens 2 ) het objectiveren van veranderingen op een systematische wijze.

Het verkrijgen van data zou volgens een nieuwe standaard moeten gebeuren. Ten eerste is het belangrijk dat ECG-geleide CTA scans de standaard worden. De hoge resolutie en adequate timing van beeldopname geeft de meest accurate anatomische informatie. In dit proefschrift werd al duidelijk dat de waarde van morfologische beoordeling en de beoordeling van aorta interventies sterk samenhangt met hemodynamische data en dat anatomie alleen te weinig is voor een adequate uitgebreide endovasculaire analyse. Toekomstig wetenschappelijk werk moet daarom hemodynamische aorta analyse kunnen verrichten, waarbij flowmetingen en drukmetingen essentieel zijn. Voor de flowmetingen is 4 dimensionele magnetic resonance imaging (MRI) geschikt. Doppler echo wordt 
als non-invasieve meting reeds minder nauwkeurig en beoefenaarsafhankelijk geacht. Naast anatomie en flow is het derde deel van de puzzel tensie. Zonder adequate tensiemetingen zijn analyses rondom endovasculaire therapie aanzienlijk minder relevant. Ondanks geavanceerde ontwikkelingen zoals intravasculaire drukkatheters, tonometrie of arterielijnen, blijt de meest robuuste en hanteerbare optie sfygmomanometrie ter plaatse van de arteria brachialis. Helaas worden deze drukken in de praktijk niet overal op de juiste en identieke manier gemeten: liggend aan dezelfde arm en op het moment van de MRI of CT scan. Voorts blijft het van belang zich te realiseren dat armdrukmetingen geen perfecte afspiegeling van de centrale aorta druk zijn. ${ }^{51}$ Toch zou de bovenstaande standaard manier van sfygmomanometrie een eenvoudige oplossing zijn om klinisch en wetenschappelijk werk rondom aorta pathologie aanzienlijk te verbeteren.

Vervolgens is er behoefte aan een systematische manier van het verwerken van onze data. Hoofdstuk 3 toont dat diameters, toegepast bij diagnose en behandeling, een foutief beeld van de aorta kunnen vormen, terwijl oppervlakte betrouwbaarder lijkt. Computationele analyses worden momenteel alleen in een onderzoeksopzet toegepast, omdat voor direct klinisch gebruik nog te veel iteraties nodig zijn en omdat het proces nog te veel tijd kost en de kwaliteit nog afhankelijk is van de ervaring van de computatie- en opslagmogelijkheden en van de ervaring van de modelbouwer. Daarentegen biedt het absoluut klinische applicatiemogelijkheden voor de nabije toekomt, middels non-invasieve informatie omtrent hemodynamica in complexe anatomie en chirurgische cardiovasculaire planning. Het is belangrijk om een geautomatiseerd werkschema te hebben met gelijke definities en gelijke modellen. Daarom is een vloeistof-structuur interactie model met patiënt specifieke anatomie tegenwoordig de standaard. In afwachting van deep learning voor het maken van de anatomische modellen op basis van beeldvorming en voor het uitvoeren van de computaties, is het belangrijk om gebreken te limiteren. Dit werd in het eerste punt met dataverzameling al aangehaald, maar het geldt ook voor het verwerken van data. Hiervoor is een continue samenwerking tussen medische bedrijven, ingenieurs en artsen noodzakelijk, om translationeel biomechanisch onderzoek te incorporeren in de kliniek. Dit kan leiden tot verbetering in de ontwikkeling van grafts en adjuncten. Hoewel enig hemodynamisch en biomechanisch effect ten gevolge van stijfheids mismatch onvermijdelijk lijkt, kan hier mogelijk winst geboekt worden, zoals beschreven in hoofdstuk 7 en 9.

Tenslotte is er een tekort aan goede rapportage en een tekort aan lange-termijn resultaten. ${ }^{52}$ Een belangrijk punt dat aandacht behoeft is de matige follow-up van patiënten als gevolg van centralisatie van gespecialiseerde zorg. Patiënten wonend in buitengebieden of met een matige sociaal-economische status hebben niet de middelen om adequaat gevolgd te worden in nabije kleiner wordende centra. Om de kwaliteit van de dataverza- 
meling te bevorderen bij grote centra en bij satelliet centra moeten er toegewijde data managers worden aangenomen in veel gebieden. Data managers hebben als taak om niet alleen data te verzamelen, maar ook bij te dragen aan ontwikkelingen van geprotocolleerde en veilige infrastructuur van dataverzameling, -uitwisseling en -opslag. Dit creëert een grotere betrokkenheid aan grotere studieprojecten, meer klinische impact, waarbij dankzij het groepsgevoel makkelijker de gezamenlijke doelen behaald kunnen worden. 


\section{REFERENCES}

1. Hagan PG, Nienaber CA, Isselbacher EM, et al. The International Registry of Acute Aortic Dissection (IRAD): new insights into an old disease. JAMA. 2000;283(7):897-903.

2. Conway BD, Stamou SC, Kouchoukos NT, et al. Improved clinical outcomes and survival following repair of acute type A aortic dissection in the current era. Interact Cardiovasc Thorac Surg. 2014;19(6):971-976. doi:10.1093/ icvts/ivu268

3. Pape LA, Awais M, Woznicki EM, et al. Presentation, diagnosis, and outcomes of acute aortic dissection: 17year trends from the international registry of acute aortic dissection. J Am Coll Cardiol. 2015;66(4):350-358. doi:10.1016/j.jacc.2015.05.029

4. Yang B, Rosati CM, Norton EL, et al. Endovascular Fenestration/Stenting First Followed by Delayed Open Aortic Repair for Acute Type A Aortic Dissection with Malperfusion Syndrome. Circulation. 2018;138(19):2091-2103. doi:10.1161/CIRCULATIONAHA.118.036328

5. Wang GJ, Jackson BM, Foley PJ, et al. National trends in admissions, repair, and mortality for thoracic aortic aneurysm and type B dissection in the National Inpatient Sample. J Vasc Surg. 2018;67(6):1649-1658. doi:10.1016/j.jvs.2017.09.050

6. Semba CP, Mitchell RS, Miller DC, et al. Thoracic aortic aneurysm repair with endovascular stent-grafts. VasC Med. 1997;2(2):98-103. doi:10.1177/1358863X9700200205

7. Patel HJ, Shillingford MS, Mihalik S, Proctor MC, Deeb GM. Resection of the Descending Thoracic Aorta: Outcomes After Use of Hypothermic Circulatory Arrest. Ann Thorac Surg. 2006;82(1):90-96. doi:10.1016/j.athoracsur.2006.02.055

8. Chiu P, Goldstone AB, Schaffer JM, et al. Endovascular Versus Open Repair of Intact Descending Thoracic Aortic Aneurysms. J Am Coll Cardiol. 2019;73(6):643-651. doi:10.1016/j.jacc.2018.10.086

9. Riambau V, Böckler D, Brunkwall J, et al. Management of Descending Thoracic Aorta Diseases: Clinical Practice Guidelines of the European Society for Vascular Surgery (ESVS). Eur J Vasc Endovasc Surg. 2017;53(1):4-52. doi:10.1016/j.ejvs.2016.06.005

10. Hiratzka LF, Bakris GL, Beckman JA, et al. 2010 ACCF/AHA/AATS/ACR/ASA/SCA/SCAI/SIR/STS/SVM Guidelines for the Diagnosis and Management of Patients with Thoracic Aortic Disease. Anesth Analg. 2010;111(2):279-315. doi:10.1016/j.jacc.2010.02.010

11. Erbel R, Aboyans V, Boileau C, et al. 2014 ESC guidelines on the diagnosis and treatment of aortic diseases. Russ J Cardiol. 2015;123(7):7-72. doi:10.15829/1560-4071-2015-07-7-72

12. Abraha I, Romagnoli C, Montedori A, Cirocchi R. Thoracic stent graft versus surgery for thoracic aneurysm. Cochrane Database Syst Rev. 2016;2016(6). doi:10.1002/14651858.CD006796.pub4

13. Andersen ND, Ganapathi AM, Hanna JM, Williams JB, Gaca JG, Hughes GC. Outcomes of acute type A dissection repair before and after implementation of a multidisciplinary thoracic aortic surgery program. J Am Coll Cardiol. 2014;63(17):1796-1803. doi:10.1016/j.jacc.2013.10.085

14. Bashir M, Harky A, Fok M, et al. Acute type A aortic dissection in the United Kingdom: Surgeon volume-outcome relation. J Thorac Cardiovasc Surg. 2017;154(2):398-406.e1. doi:10.1016/j.jtcvs.2017.02.015

15. Smith HN, Boodhwani M, Ouzounian M, et al. Classification and outcomes of extended arch repair for acute Type A aortic dissection: A systematic review and meta-analysis. Interact Cardiovasc Thorac Surg. 2017;24(3):450459. doi:10.1093/icvts/ivw355

16. Halstead JC, Meier M, Etz C, et al. The fate of the distal aorta after repair of acute type A aortic dissection. J Thorac Cardiovasc Surg. 2007;133(1):127-135. doi:10.1016/j.jtcvs.2006.07.043

17. Leontyev S, Haag F, Davierwala PM, et al. Postoperative Changes in the Distal Residual Aorta after Surgery for Acute Type A Aortic Dissection: Impact of False Lumen Patency and Size of Descending Aorta. Thorac Cardiovasc Surg. 2017;65(2):90-98. doi:10.1055/s-0036-1571813

18. Bojko MM, Suhail M, Bavaria JE, et al. Midterm outcomes of emergency surgery for acute type A aortic dissection in octogenarians. In: Journal of Thoracic and Cardiovascular Surgery. Vol 0. Mosby Inc.; 2020. doi:10.1016/j. jtcvs.2020.03.157 
Discussion

19. Jussli-Melchers J, Panholzer B, Friedrich C, et al. Long-term outcome and quality of life following emergency surgery for acute aortic dissection type A: A comparison between young and elderly adults. Eur J Cardio-thoracic Surg. 2017;51(3):465-471. doi:10.1093/ejcts/ezw408

20. Tang GHL, Malekan R, Yu CJ, Kai M, Lansman SL, Spielvogel D. Surgery for acute type A aortic dissection in octogenarians is justified. J Thorac Cardiovasc Surg. 2013;145(3 SUPPL.):S186-S190. doi:10.1016/j.jtcvs.2012.11.060

21. Trimarchi S, Eagle KA, Nienaber CA, et al. Role of age in acute type A aortic dissection outcome: Report from the International Registry of Acute Aortic Dissection (IRAD). J Thorac Cardiovasc Surg. 2010;140:784-789. doi:10.1016/j.jtcvs.2009.11.014

22. Santini F, Montalbano G, Messina A, et al. Survival and quality of life after repair of acute type A aortic dissection in patients aged 75 years and older justify intervention. Eur J Cardio-thoracic Surg. 2006;29(3):386-391. doi:10.1016/j.ejcts.2005.12.016

23. Aoyama T, Kunisawa S, Fushimi K, Sawa T, Imanaka Y. Comparison of surgical and conservative treatment outcomes for type a aortic dissection in elderly patients. J Cardiothorac Surg. 2018;13(1):1-8. doi:10.1186/ s13019-018-0814-6

24. Heijmen R, Fattori R, Thompson M, et al. Mid-term outcomes and aortic remodelling after thoracic endovascular repair for acute, subacute, and chronic aortic dissection: The VIRTUE Registry. Eur J Vasc Endovasc Surg. 2014;48(4):363-371. doi:10.1016/j.ejvs.2014.05.007

25. Patel HJ, Williams DM, Upchurch GR, et al. A Comparison of Open and Endovascular Descending Thoracic Aortic Repair in Patients Older Than 75 Years of Age. Ann Thorac Surg. 2008;85(5):1597-1604. doi:10.1016/j.athoracsur.2008.01.044

26. Kim JB, Lee CH, Lee TY, et al. Descending aortic aneurysmal changes following surgery for acute DeBakey type I aortic dissection. Eur J Cardio-thoracic Surg. 2012;42(5):851-857. doi:10.1093/ejcts/ezs157

27. Stanley GA, Murphy EH, Knowles M, et al. Volumetric analysis of type B aortic dissections treated with thoracic endovascular aortic repair. J Vasc Surg. 2011;54(4):985-992. doi:10.1016/j.jvs.2011.03.263

28. Tolenaar JL, Kern JA, Jonker FH, et al. Predictors of false lumen thrombosis in type B aortic dissection treated with TEVAR. Ann Cardiothorac Surg. 2014;3(3):255-263. doi:10.3978/j.issn.2225-319X.2014.05.17

29. Patel HJ, Williams DM, Drews JD, et al. A 20-year experience with thoracic endovascular aortic repair. Ann Surg. 2014;260(4):691-697. doi:10.1097/SLA.0000000000000930

30. Ziza V, Canaud L, Molinari N, Branchereau P, Marty-Ané C, Alric P. Thoracic endovascular aortic repair: A single center's 15-year experience. J Thorac Cardiovasc Surg. 2016;151(6):1595-1603.e7. doi:10.1016/j. jtcvs.2015.12.030

31. Wiedemann D, Mahr S, Vadehra A, et al. Thoracic endovascular aortic repair in 300 patients: Long-term results. Ann Thorac Surg. 2013;95(5):1577-1583. doi:10.1016/j.athoracsur.2013.02.043

32. DeMartino RR, Sen I, Huang Y, et al. Population-Based Assessment of the Incidence of Aortic Dissection, Intramural Hematoma, and Penetrating Ulcer, and Its Associated Mortality From 1995 to 2015. Circ Cardiovasc Qual Outcomes. 2018;11(8):e004689. doi:10.1161/CIRCOUTCOMES.118.004689

33. Von Kodolitsch Y, Csösz SK, Koschyk DH, et al. Intramural Hematoma of the Aorta Predictors of Progression to Dissection and Rupture. Circulation. 2003;107:1158-1163. doi:10.1161/01.CIR.0000052628.77047.EA

34. Belvroy VM, de Beaufort HWL, van Herwaarden JA, Trimarchi S, Moll FL, Bismuth J. Type 1b Endoleaks After Thoracic Endovascular Aortic Repair are Inadequately Reported: A Systematic Review. Ann Vasc Surg. 2019. doi:10.1016/j.avsg.2019.06.030

35. Baguet JP, Chavanon O, Sessa C, et al. European Society of Hypertension scientific newsletter: Hypertension and aortic diseases. J Hypertens. 2012;30(2):440-443. doi:10.1097/HJH.0B013E32834F867A

36. Cregler L. Aortic dissection and cocaine use. Am Heart J. 1992;124(6):1665. doi:10.1016/0002-8703(92)90109-9

37. Nienaber CA, Clough RE. Management of acute aortic dissection. Lancet. 2015;385(9970):800-811. doi:10.1016/ S0140-6736(14)61005-9

38. Trimarchi S, Jonker FHW, Froehlich JB, et al. Acute type B aortic dissection in the absence of aortic dilatation. J Vasc Surg. 2012;56(2):311-316. doi:10.1016/j.jvs.2012.01.055

39. Berezowski M, Kosiorowska K, Beyersdorf F, et al. Modelling of predissection aortic size in acute descending aortic dissection. Interact Cardiovasc Thorac Surg. 2019;29(1):124-129. doi:10.1093/icvts/ivz028 
40. Cyron CJ, Wilson JS, Humphrey JD. Mechanobiological stability: A new paradigm to understand the enlargement of aneurysms? J R Soc Interface. 2014;11(100). doi:10.1098/rsif.2014.0680

41. Spadaccio C, Nappi F, Al-Attar N, et al. Old Myths, New Concerns: the Long-Term Effects of Ascending Aorta Replacement with Dacron Grafts. Not All That Glitters Is Gold. J Cardiovasc Transl Res. 2016;9(4):334-342. doi:10.1007/s12265-016-9699-8

42. Ioannou C V., Stergiopulos N, Katsamouris AN, et al. Hemodynamics induced after acute reduction of proximal thoracic aorta compliance. Eur J Vasc Endovasc Surg. 2003;26(2):195-204. doi:10.1053/ejvs.2002.1917

43. Roccabianca S, Figueroa CA, Tellides G, Humphrey JD. Quantification of regional differences in aortic stiffness in the aging human. J Mech Behav Biomed Mater. 2014;29:618-634. doi:10.1016/j.jmbbm.2013.01.026

44. Tremblay D, Zigras T, Cartier R, et al. A Comparison of Mechanical Properties of Materials Used in Aortic Arch Reconstruction. Ann Thorac Surg. 2009;88(5):1484-1491. doi:10.1016/j.athoracsur.2009.07.023

45. Nauta FJH, Conti M, Marconi S, et al. An experimental investigation of the impact of thoracic endovascular aortic repair on longitudinal strain. Eur J Cardio-thoracic Surg. 2016;50(5):955-961. doi:10.1093/ejcts/ezw180

46. Nauta FJH, de Beaufort HWL, Conti M, et al. Impact of thoracic endovascular aortic repair on radial strain in an ex vivo porcine model. Eur J Cardio-thoracic Surg. 2017;51(4):783-789. doi:10.1093/ejcts/ezw393

47. Nauta FJH, Van Bogerijen GHW, Trentin C, et al. Impact of thoracic endovascular aortic repair on pulsatile circumferential and longitudinal strain in patients with aneurysm. J Endovasc Ther. 2017;24(2):281-289. doi:10.1177/1526602816687086

48. De Beaufort HWL, Coda M, Conti M, et al. Changes in aortic pulse wave velocity of four thoracic aortic stent grafts in an ex vivo porcine model. PLoS One. 2017;12(10). doi:10.1371/journal.pone.0186080

49. de Beaufort HWL, Conti M, Kamman A V., et al. Stent-Graft Deployment Increases Aortic Stiffness in an Ex Vivo Porcine Model. Ann Vasc Surg. 2017;43:302-308. doi:10.1016/j.avsg.2017.04.024

50. van Bakel TM, Arthurs CJ, Nauta FJ, et al. Cardiac remodeling following thoracic endovascular aortic repair for descending aortic aneurysms. Eur J Cardio-Thoracic Surg. 2018;0:1-10. doi:10.1016/0038-1098(79)91043-3

51. Agabiti-Rosei E, Mancia G, O'Rourke MF, et al. Central blood pressure measurements and antihypertensive therapy: A consensus document. Hypertension. 2007;50(1):154-160. doi:10.1161/HYPERTENSIONAHA.107.090068

52. Kret MR, Azarbal AF, Mitchell EL, Liem TK, Landry GJ. From the Western Vascular Society Compliance with longterm surveillance recommendations following endovascular aneurysm repair or type B aortic dissection. J VasC Surg. 2013;58:25-32. doi:10.1016/j.jvs.2012.12.046 

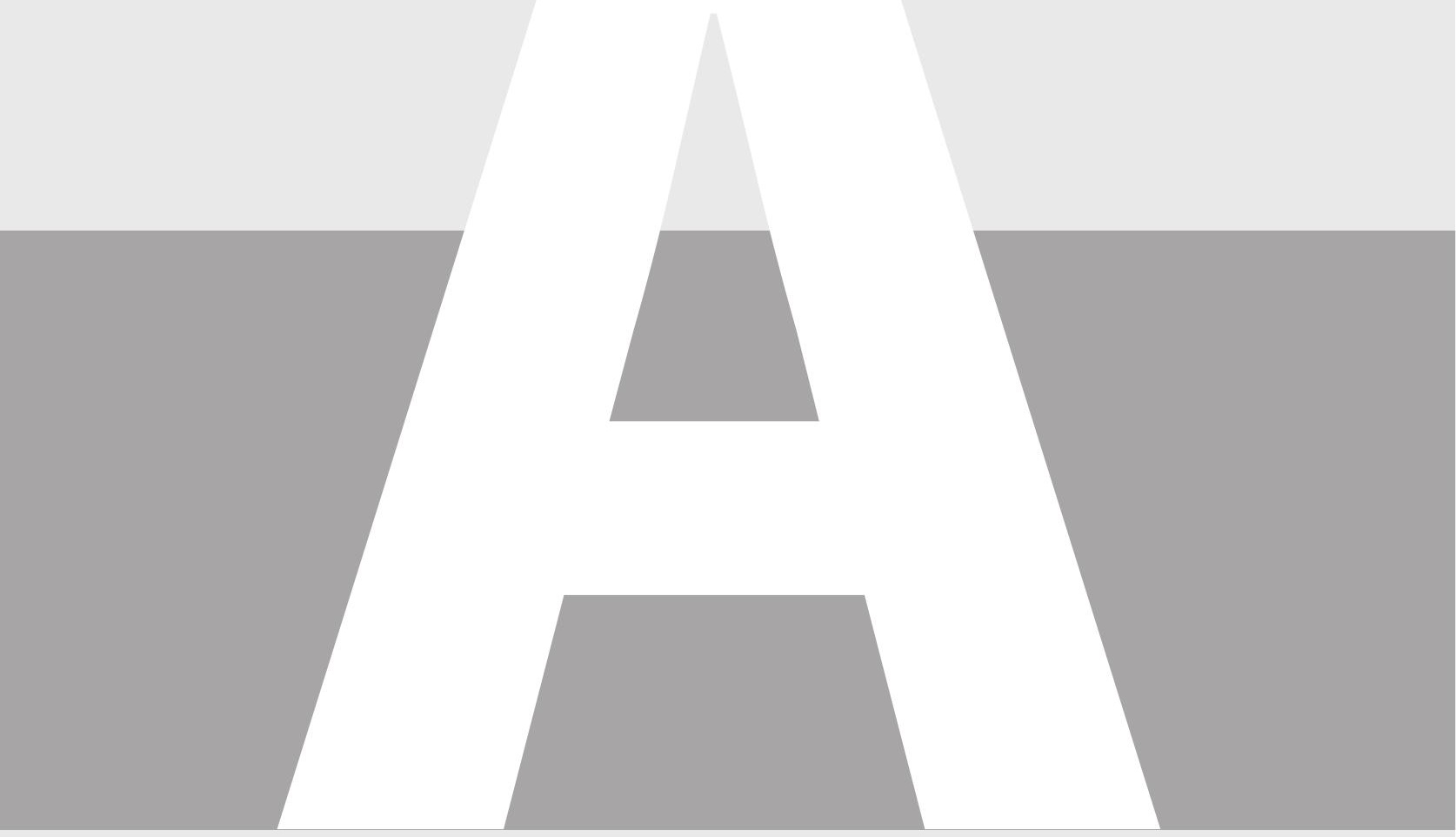
- Review committee

- Acknowledgments

- About the author 



\section{REVIEW COMMITTEE}

Prof. dr. T. Leiner

Prof. dr. J.P.P.M. de Vries

Prof. dr. D.W. Donker

Dr. R.H. Heijmen

Prof. dr. W.J.L. Suyker 



\section{ACKNOWLEDGEMENTS}

Geachte professor doctor J.A. van Herwaarden, beste Joost, bedankt voor je scherpe en kritische observaties voor de stukken, bedankt voor je support, zeker in het laatste anderhalf jaar dat ik terug was in Nederland en bovenal bedankt voor de goede gesprekken, ook de gesprekken die niet over onderzoek gingen.

Geachte professor doctor F.L. Moll, beste professor, u heeft mij laten zien wat goed mentorschap inhoudt. $U$ wist altijd precies wanneer ik wel of geen sturing nodig had. Ik waardeer onze gesprekken over de richting van het aorta-onderzoek enorm. Bedankt voor de kans en ervaring die u mij gegeven heeft en bedankt voor uw onverminderde en inspirerende enthousiasme.

Dear professor H.J. Patel, dear Himanshu, I will always cherish a warm welcoming feeling when thinking of my time in Ann Arbor, specifically at the cardiovascular center in the University of Michigan Hospital and you made that possible. The meetings at Zingerman's were the best! Thank you for your support and everything you taught me on clinical research and writing.

Dear Heena, Milan, Tejal and Kajal, the plentiful dinners with delicious Indian food have often times made me eat more than initially expected. Thank you for all the good laughs and for making me feel so at home.

Dear professor C.A. Figueroa, dear Alberto, you are as passionate about research as you are about basically everything else in life. I have come to admire the way you teach your students and I am glad I was able to be part of that teaching experience. You taught me that time is one's most valuable asset and I am glad we have shared some of our time together.

Dear Andrea, Oliver and Clara, thank you for the wonderful Thanksgiving dinners and barbecues and all your hospitality.

Dear doctor N.S. Burris, dear Nick, your drive and enthusiasm for research and imaging are admirable and catchy. I have learned a great deal from you and have always enjoyed working with you. You even sparked my interest in imaging, for which I am grateful. Dear Heather, thank you for your hospitality and the wonderful dinners. I am very glad to have met the both of you.

Aan de opzetters en eerste passagiers van de aorta-trein: Frederik, Jip, Guido, Arnoud en in het bijzonder Foeke, bedankt! Foeke we hebben een korte, maar mooie tijd samen 
gehad in het Flevoziekenhuis. Via jou is de bal voor mij gaan rollen met betrekking tot de promotie, waarvoor dank.

Squadra A, dat wil zeggen, de rest van de PhD-ers in het promotie traject, ontzettend bedankt voor al jullie support en mooie momenten. Mijn mooiste herinnering aan onze groep is onze cardiovascular course in Houston. Dat we nog vaak mogen borrelen samen!

Beste Diederik, met jou ben ik mijn onderzoekstijd begonnen in Ann Arbor. Je hebt mij veel geleerd en ik kon altijd goed met je sparren. Beste Margot, heel erg bedankt voor al je gastvrijheid. Diederik en jij waren de vaste thuishaven voor het lab buiten werk en ik voelde me bij jullie direct thuis en ik wil jullie ontzettend bedanken voor alles wat jullie voor me hebben betekend in Ann Arbor.

Beste Hector, ook al hebben we maar een maand samen gewoond in Ann Arbor, ik heb je in die tijd en ook in de tijd daarna goed leren kennen. Ik waardeer je originele mening en de dikwijls bizarre, maar interessante gesprekken. Ook in Utrecht ben je een goede vriend van me geworden. Bedankt.

Beste Viony, we hebben nooit samen op dezelfde onderzoeksplek gezeten en gek genoeg had ik direct een goede band met je alsof we elkaar al jaren kenden. We hebben mooie congressen en tripjes samen beleefd. Bedankt.

Beste Yunus, Young Yun!, vanaf het moment dat je bij mij in huis kwam wonen in Ann Arbor heb ik me geen moment verveeld. Je brengt een ongekende gemoedelijkheid en positiviteit met je mee en biedt de mogelijkheid om alles bespreekbaar te maken. Ik heb veel bewondering voor je en ben blij dat ik van je heb kunnen leren. Bedankt!

Nieuwste garde in de Squadra A: Pieter en Tim, jullie zijn al bezig met veel enthousiasme. Geniet van jullie onderzoekstijd!

Dear members of the cardiovascular biomechanics lab: Jonas, Fede, Sabrina, Chris, Kritika, Vasilina, Nitesh. Thank you for everything you have done for me. It truly felt like I was part of a research family when I was in- and outside the lab with you guys.

Dear team Henri! Dear Jon, Richard, Lauren and Lane. When I think of Ann Arbor, some of the first memories that pop into my mind are those that I share with you all in our little big house (LBH). I couldn't have wished for better friends than you. Lane, I treasure your college football commentaries, resembling professional coaching masterclasses in the eyes of a simple Dutch guy. Lauren, I fondly remember our trips to see awesome 
music performances. Richard, number one party planner. The Last Word sessions and many other good whiskey and talking sessions were memorable to me and highlighted your ability to be a good listener and conversation companion. Jon, my true Ann Arbor companion, we have had a ton of fun together, you are like a good friend and a brother to me. I could do this all day!

Dear Angel and Ilias, thank you for all your help. I have enjoyed working with the both of you and wish you all the best. You are exceptionally hard and efficient workers and I am sure you will do well in life.

Dear Nickole, Amanda, Mary, Emily, without your limitless efforts on getting studies started, maintaining them, providing us with the right information and enabling us to talk to busy physicians, none of our clinical research would be possible. More importantly, each of you brings a positive vibe to the office. Thank you!

Beste Susan, Cobie en Cynthia, hartelijk dank voor het verzorgen van alle administratieve en logistieke zaken. De samenwerking met jullie verloopt altijd prettig en zonder problemen.

Zwolle en onzestreken, dank voor jullie steun, de regelmatige belletjes en voor de mooie avonden die we de afgelopen jaren hadden. Ik prijs mijzelf gelukkig met goede vrienden als jullie waar ik altijd op terug kan vallen.

Patronés, dank voor de nodige afleiding de afgelopen jaren, waaronder een zeer memorabele lustrumreis.

Beste dr. van Grevenstein, beste Helma, dank voor je steun en het pushen om door te werken naast mijn klinische tijd in Utrecht.

Lieve Tjitske, Tijs, Sjors, Eva, Jelle en Roos, mijn lieve broers en zussen. Bedankt voor jullie afleiding en luisterend oor en steun op allerlei manieren, welke jullie altijd voor mij zijn geweest en altijd zullen zijn. Ik hou van jullie.

Lieve Charlotte, lieve Lot, wat hebben we veel meegemaakt samen. We hebben geweldige tijden in Nederland, Amerika en in vele andere landen gehad. Je bent jarenlang mijn vaste steun en toeverlaat geweest. Een lange tijd hebben we vrijwel dagelijks met elkaar gebeld. Jouw rust, jouw geduld, de spiegel die je mij dikwijls voor hield en je pragmatische kijk op zaken zijn bewonderenswaardig. Deze promotie is voor een goed deel van jou. 
Lieve Pap en Mam, jullie hebben mij mijn hele leven onvoorwaardelijk gesteund. Mijn ontwikkelingen als persoon zijn voor een groot deel resultaat van jullie voorbeeld en begeleiding. Ik ben trots op jullie en het voelt nog altijd als heerlijk thuis komen wanneer ik in Zwolle langs kom. Bedankt voor alles wat jullie voor mij betekend hebben. Ik hou van jullie! 


\section{ABOUT THE AUTHOR}

Ignas Bernardus Houben was born on April 261991 in Maastricht, the Netherlands. During his medical school, amongst several extracurricular activities, he was part of the executive board for the International Student Congress of (bio)Medical Sciences. He obtained his medical degree from the University of Groningen in 2016 and started working as a General Surgery resident at the Flevoziekenhuis in Almere. After getting in touch with prof. dr. F.L. Moll and prof. dr. J.A. van Herwaarden, he was invited to conduct research at the Department of Vascular Surgery of the University Medical Center Utrecht. The primary focus would be to assess clinical outcomes in aortic surgery and to assess morphologic features in preoperative planning and postsurgical evaluation, with an emphasis on endovascular treatment. Ignas started his research in Ann Arbor, MI, USA, at the Cardiovascular Center of the University of Michigan Hospital during the summer of 2017. He worked under prof. dr. H.J. Patel to gain scientific information on the clinical and biomechanical understanding of aortic diseases and its treatment. He did so in a multidisciplinary team in close collaboration with prof. dr. C.A. Figueroa and dr. N.S. Burris. The research with a large focus on endovascular treatment and morphologic assessment sparked an interest in interventional radiology. After returning to the University Medical Center Utrecht, Ignas continued his clinical work as a General Surgery resident, combined with final work on his research. At the end of 2020, Ignas moved to Maastricht to gain clinical experience in a large peripheral center in Surgical Residency, the Zuyderland Medical Center, all the while keeping a keen interest in interventional radiology and vascular surgery. Recently, Ignas started as a resident in training to become a radiologist at the Isala hospital in Zwolle. In the meantime, he continues to work on aortic research, looking to further the scientific and clinical knowledge in minimally invasive vascular procedures. 